\title{
DISCOVERY AND DIRECTED EVOLUTION OF NITROREDUCTASE ENZYMES FOR ACTIVATION OF PRODRUGS AND PET IMAGING COMPOUNDS
}

BY

\section{MICHELLE HEDLEY RICH}

\author{
A thesis \\ submitted to the Victoria University of Wellington \\ in fulfilment of the requirements for the degree of \\ Doctor of Philosophy
}

Victoria University of Wellington

(2017) 



\section{Abstract}

Bacterial nitroreductase enzymes, which exhibit the capacity to reduce a wide range of nitroaromatic drugs, antibiotics and environmental pollutants, have shown promise in the activation of prodrugs such as CB1954 and PR-104A. Use of these prodrugs in gene-directed enzyme prodrug therapy (GDEPT) cancer treatment would allow for targeted chemotherapy in tumour cells following specific delivery of nitroreductases to these cancerous tissues, using specialised bacterial or viral vectors. However, one key limitation in nitroreductase-based GDEPT is the current inability to rapidly and non-invasively determine vector localisation and gene delivery prior to systemic administration of prodrug. Dual-purpose nitroreductases that exhibit the ability to activate both GDEPT prodrugs and radioisotope-labelled PET imaging probes, in a manner that renders them temporarily cell-entrapped for detection using a PET scanner, would facilitate clinical development of this treatment.

Previous attempts to repurpose hypoxia-activated 2-nitroimidazole PET imaging probes for nitroreductase detection have suffered from relatively high background activation under hypoxia alone. The design of nextgeneration 5-nitroimidazole PET imaging probes, by our collaborators at the Auckland Cancer Society Research Centre (ACSRC), has resulted in much lower levels of hypoxia activation in vivo.

This thesis describes attempts to generate improved nitroreductases that can activate a bespoke 5-nitroimidazole PET-capable imaging probe, S33. A 58-membered library of nitroreductase candidates, including enzymes from many different bacterial species and oxidoreductase families, was heterologously over-expressed in E. coli screening strains. Microplate-based screening strategies were then used to identify enzymes that exhibited the most activity with S33, based on the ability of high levels of activated S33 to induce DNA damage and (at very high levels) E. coli cell death. Following this, site-targeted libraries of two different promising nitroreductase NfsA homologues were screened for S33 activity, with selected variants from each 
library showing improvement in S33 activation over the parent nitroreductase. In parallel I performed error-prone PCR mutagenesis of a top NfsA variant and top NfsB variant, subjecting each to two rounds of random mutagenesis, and selecting improved variants using a specialised $E$. coli screening strain and fluorescence-activated cell sorting (FACS). Selected variants from the NfsB (but not NfsA) nitroreductase candidate library showed substantially improved capacity to activate S33 over the parent enzyme.

As an alternative means for developing improved nitroreductase variants, two different nitroaromatic 'anti-prodrugs', the anthelmintic niclosamide and the antibiotic chloramphenicol, whose cytotoxic effects on E. coli can be mitigated by the presence of an over-expressed active nitroreductase, were used to select for improved S33-activating enzymes from a site-targeted NfsA library. Variants were discovered that exhibited improved ability to active S33 as well as other nitroaromatic substrates of interest.

Finally, attempts to discover novel nitroreductases from nature through the screening of cloned soil metagenomic fragments, were made utilising a novel cloning strategy to improve expression of the cloned gene fragments in E. coli. Screening and selection of nitroreductase gene fragments was conducted using niclosamide as well as nitroaromatic compounds that change colour upon activation. 


\section{Acknowledgements}

Firstl I would like to thank both the Cancer Society of New Zealand ad Victoria University of Wellington for their funding, without which this $\mathrm{PhD}$ could not have been completed.

Secondly, thanks Dave, for taking a chance on an Honours kid and somehow roping her into staying in the lab for another 4 years and counting... Thank you for both being extremely supportive as well as taking any possible opportunity to mock me, for two great conferences, and for allowing me to have a really great time studying nitroretardases all this time.

Thanks to all past and present members of the Ackerley lab and friends from other labs, especially everyone in Becky's memorial office for their jokes and laughter over these past few years. It's going to be the end of an era once we move into the new building, although I won't miss the threat of being killed by roof sealant at any moment. Thanks for all of your attempts to dissuade me from being the best lab manager the world has ever known. Though they're now overseas, a special thanks to both Elsie and Rory for all their help over the years and their participation in an unforgettable road-trip across the US, complete with amazing peaches and the worst GPS system in the world.

Thanks to all my friends and family, thank you Mama and Baba who never quite understood why I was crazy enough to do a $\mathrm{PhD}$ but supported me nonetheless. And I forgive you for replacing me with a dog. Thanks Tim for putting up with me being busy for so many years and persuading me to have fun every once in a while. Maybe I'll start kicking your arse in the gym again now that I have all this free time. 


\section{Table of Contents}

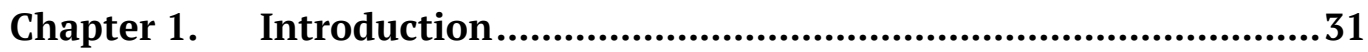

$1.1 \quad$ Targeted novel anti-cancer therapeutics ..................................... 31

1.1.1 Oncolytic viruses as targeted anti-cancer therapeutics ....................32

1.1.1.1 Talimogene laherparepvec ( $T$-Vec; or Imlygic ${ }^{\circledR}$, formerly Oncovex $\left.{ }^{G M-C S F}\right)$...33

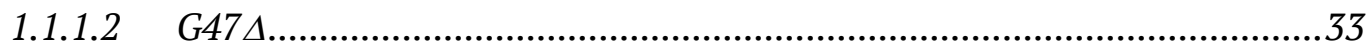

1.1.1.3 JX-594 (Pexastimogene devacirepvec; Pexa-Vec) ....................................34

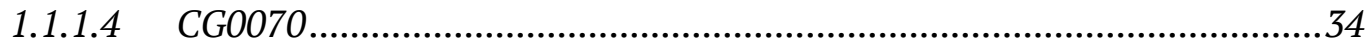

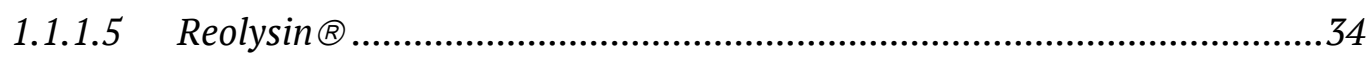

1.1.2 Bacteria as targeted anti-cancer therapeutics ............................... 35

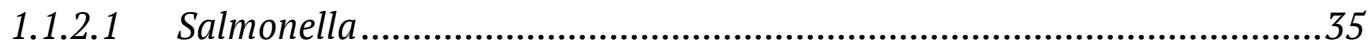

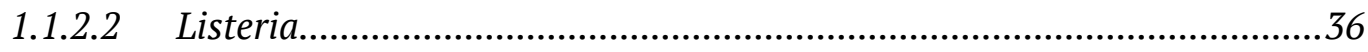

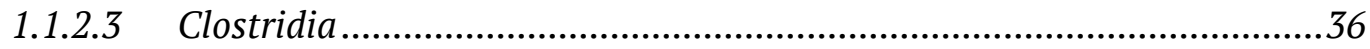

1.2 Gene directed enzyme prodrug therapy ........................................ 37

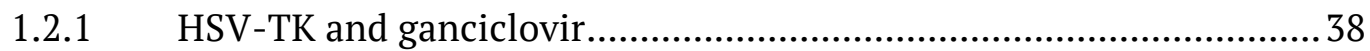

1.2.2 Cytosine deaminase/5-fluorocytosine .............................................39

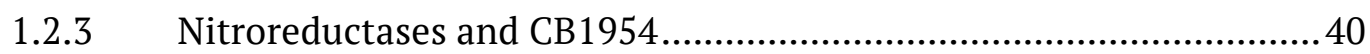

$1.3 \quad$ Nitroreductase enzymes ............................................................ 41

$1.4 \quad$ Strategies to improve nitroreductase based GDEPT ...................... 44

1.4.1 Alternative bacterial nitroreductases for GDEPT..............................44

1.4.2 Directed evolution of bacterial nitroreductases for GDEPT ...............46

1.4.3 Alternative nitroaromatic prodrugs ............................................... 48

Non-invasive imaging strategies .............................................. 51

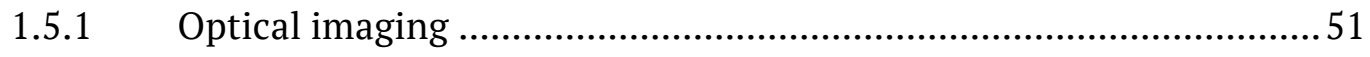

1.5.2 Magnetic resonance imaging (MRI) …...........................................5 52 


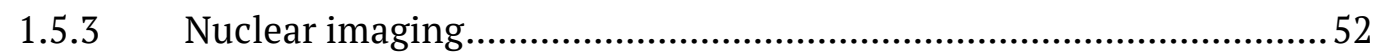

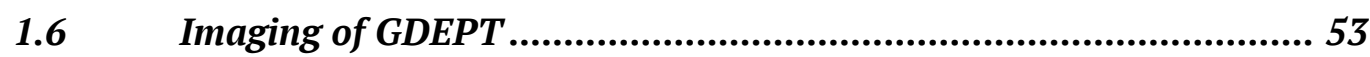

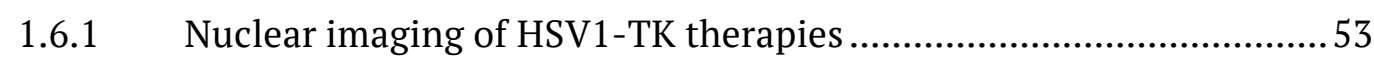

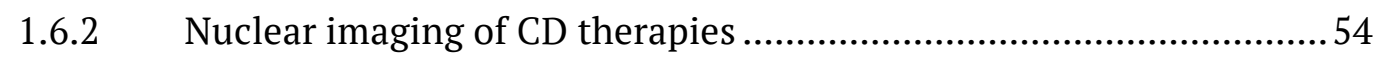

1.6.3 Optical imaging of nitroreductases............................................. 54

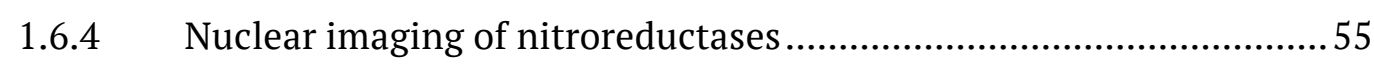

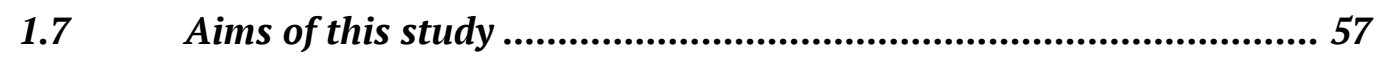

Chapter 2. Methods ..............................................................59

$2.1 \quad$ Chemical reagents and enzymes..............................................59

$2.2 \quad$ Oligonucleotide primers ..........................................................59

2.3 Software used for creation of figures, sequence alignments and enzyme active site modelling .....................................................................6 61

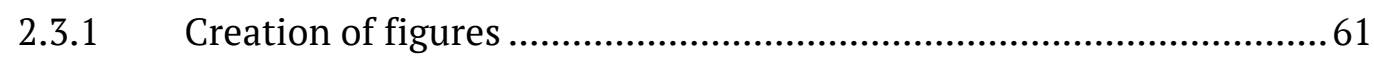

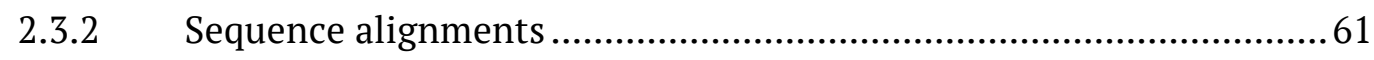

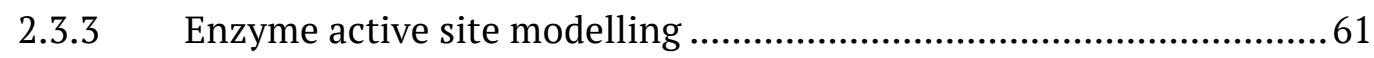

2.4 Strains and plasmids used in this study .................................62 62

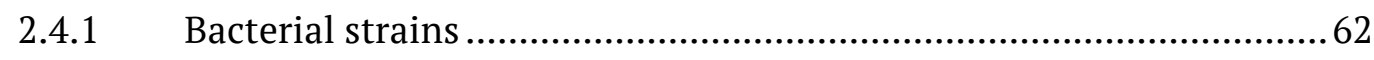

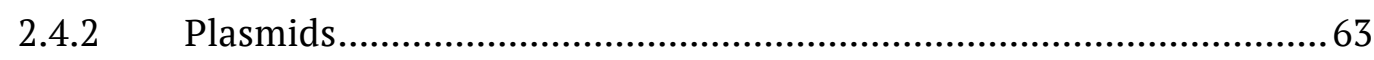

2.4.2.1 Plasmid map of expression vector $p U C X: A z o R_{-}$Ec (high expression)......64

2.4.2.2 Plasmid map and multiple-cloning site of expression vector pUCX (low

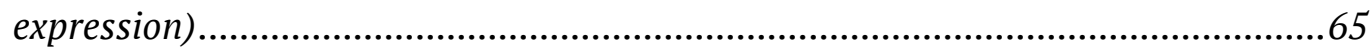

2.4.2.3 Multiple-cloning site of E. coli expression vector $p U C X$ (high expression) ..67

2.4.2.4 Plasmid map and multiple-cloning site of E. coli SOS plasmid pANODuet-1:GFP. .68

2.4.2.5 Plasmid map and multiple-cloning site of E. coli expression vector pET28a+ .69 
$2.5 \quad$ Bacterial growth and maintenance ......................................... 71

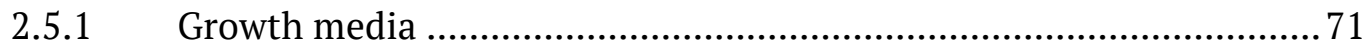

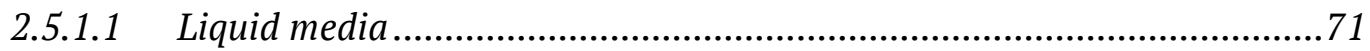

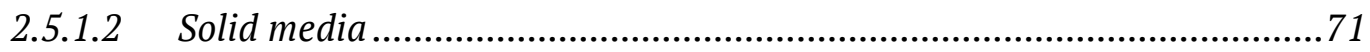

2.5.2 Bacterial antibiotics and induction agents ................................... 71

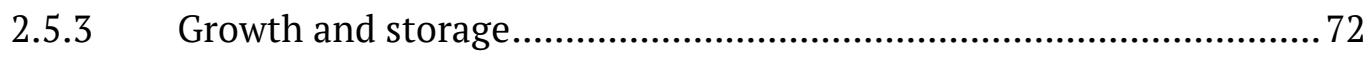

$2.6 \quad$ Standard molecular biology protocols...................................... 72

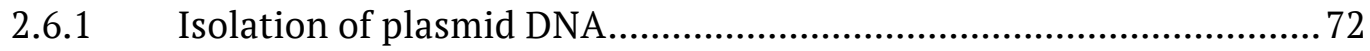

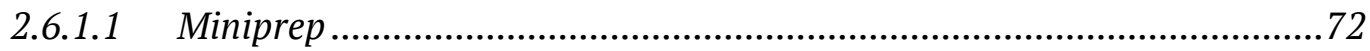

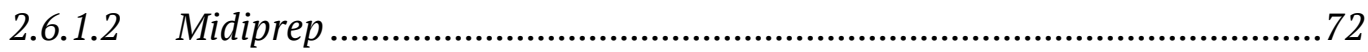

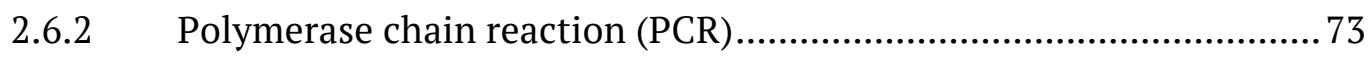

2.6.2.1 PCR components and protocol using Biomix Red .................................73

2.6.2.2 $\quad$ PCR components and protocol using Phusion ${ }^{\mathrm{TM}}$.....................................73

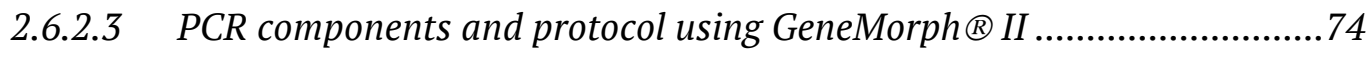

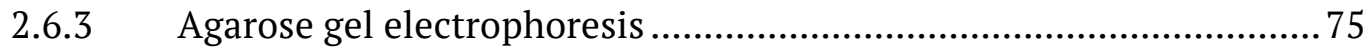

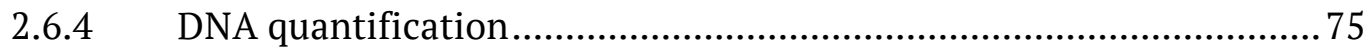

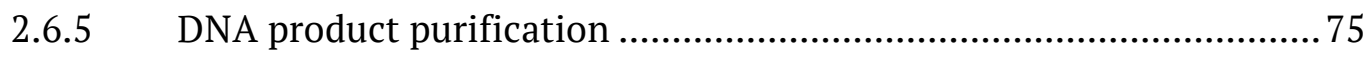

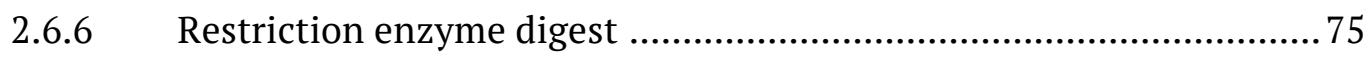

2.6.6.1 Restriction digest for mutant gene libraries..........................................76

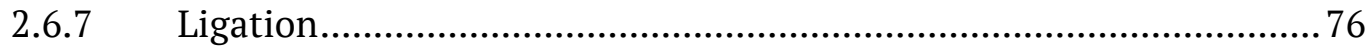

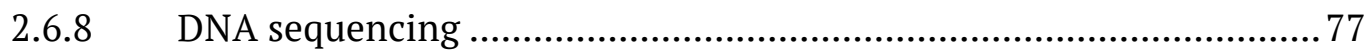

2.6.9 Transformation of competent E. coli............................................. 77

2.6.9.1 Preparation of chemically competent cells .........................................77

2.6.9.2 Transformation of chemically competent cells ......................................77

2.6.9.3 Preparation of electrocompetent cells ..................................................78

2.6.9.4 Transformation of electrocompetent cells ..........................................79 
2.6.9.5 Recovery of electrocompetent cells transformed with library DNA ..........79

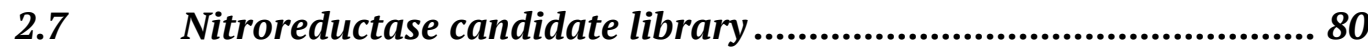

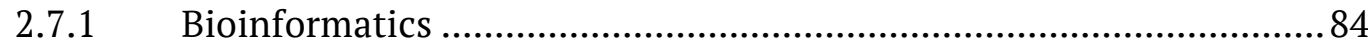

2.7.2 Expanded nitroreductase family tree ........................................... 84

$2.8 \quad N f S A \_E c 7 S M$ site saturation mutagenesis library ........................ 85

2.9 Overlap PCR for targeted amino acid substitution of a single site

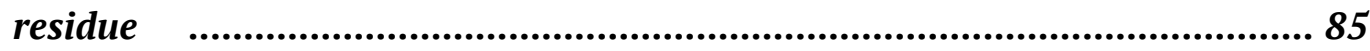

$2.10 \quad$ Generation of error-prone nitroreductase libraries.......................8 87

2.10.1 PCR creation and cloning of error-prone libraries ........................... 87

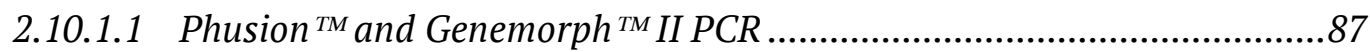

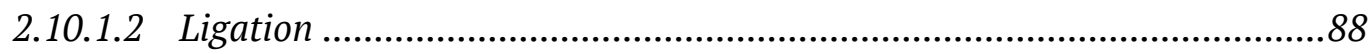

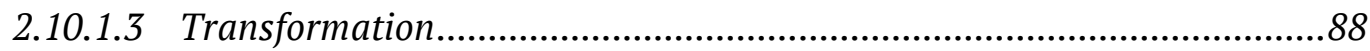

2.11 Selection of nitroreductase libraries using positive selection

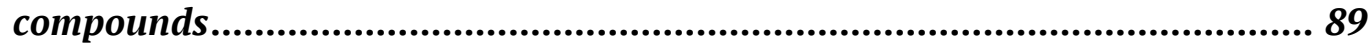

2.11.1 Niclosamide selection of nitroreductase libraries in SOS-R4 cell strain 89

2.11.2 Niclosamide or chloramphenicol selection of nitroreductase libraries

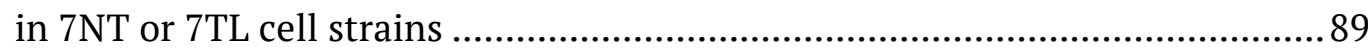

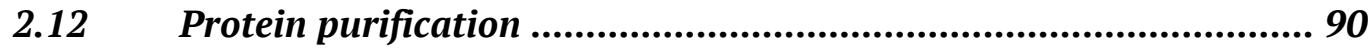

2.12.1 Expression using low temperature LB + supplements ......................99

2.12.2 Enzyme purification using $\mathrm{His}_{6}$-tag and Ni-NTA column ..................99

2.12.3 Enzyme concentration determination ........................................... 92

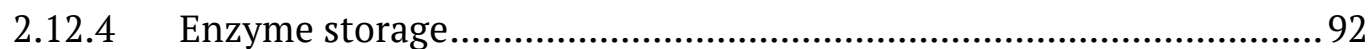

In vitro purified protein kinetics ................................................ 92

2.13.1 Determination of S33 and NADPH extinction coefficients ................92

2.13.2 Glucose oxidase-catalase oxygen scavenging system ......................93

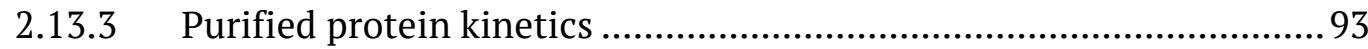


2.14 Sodium Dodecyl Sulphate Polyacrylamide Gel Electrophoresis (SDS-

PAGE)

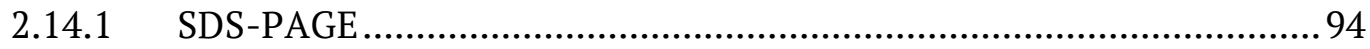

2.14.1.1 SDS-PAGE of His-tag purified proteins for enzyme kinetics ...................95

2.14.1.2 SDS-PAGE of intracellular over-expressed nitroreductases...................95

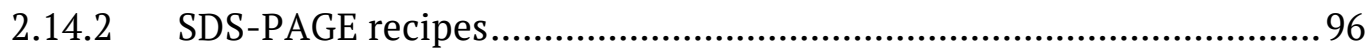

2.15 Assays for nitroreductase activity with nitroaromatic substrates .. 96

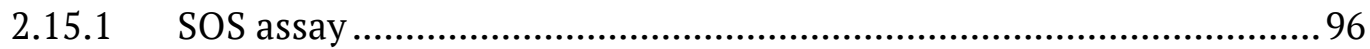

2.15.1.1 SOS assay using a lacZ reporter in SOS-R2 ....................................96

2.15.1.2 SOS assay using a GFP reporter in SOS-R4......................................98

2.15.2 Fluorescence-activated cell sorting (FACS) of nitroreductase libraries .....

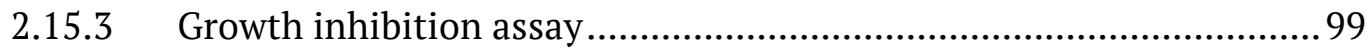

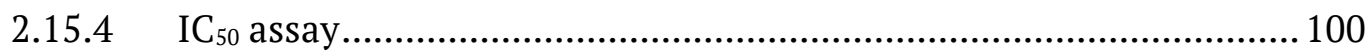

2.15.5 Biological and technical replicates ............................................. 100

$2.16 \quad$ Creation of metagenomic libraries ........................................... 101

2.16.1 Preparation of genomic DNA ................................................... 101

2.16.2 Electro-elution of restriction enzyme-cleaved genomic DNA ......... 101

2.16.3 Top agarose selection of metagenomic libraries........................... 102

Chapter 3. Screening of native and targeted-mutagenesis libraries for nitroreductases capable of metabolising S33 ....................................103

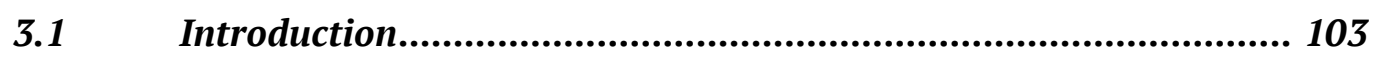

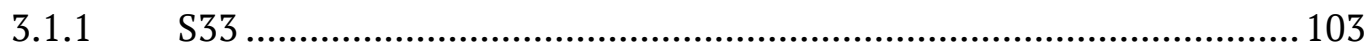

3.1.2 Expanded nitroreductase candidate library .................................. 106

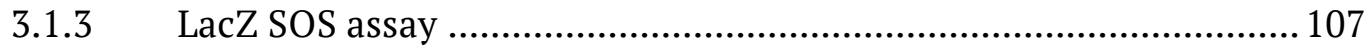

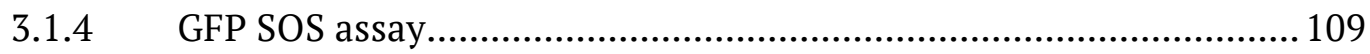


3.1.5 Bacterial nitroreductase expression and stability in human cell lines. 110

3.1.6 Nitroreductase variant libraries previously created by the Ackerley group

3.1.6.1 YcnD_Bs site-saturation mutagenesis (SSM) library............................112

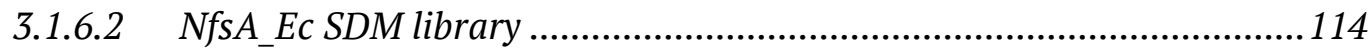

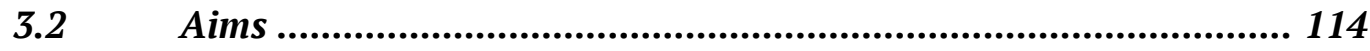

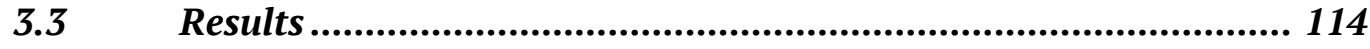

3.3.1 pUCX high and low-expressing plasmids .................................... 114

3.3.1.1 Difference in expression between low-expressing and high-expressing pUCX

3.3.2 Evaluation of wild-type nitroreductase activity with S33 ............... 120

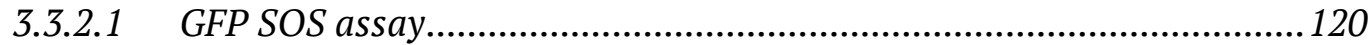

3.3.2.2 Growth inhibition $\left(I C_{50}\right)$ assays with select candidates from the 58 nitroreductase library and S33

3.3.2.3 Correlation of GFP SOS response and growth inhibition ......................123

3.3.2.4 Glucose oxidase-catalase oxygen scavenging for kinetics .....................124

3.3.2.5 Validation of the glucose oxidase-catalase oxygen scavenging system

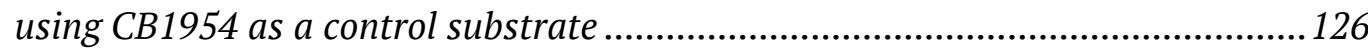

3.3.2.6 Reduction of S33 using the glucose oxidase-catalase oxygen scavenging system and $\mathrm{His}_{6}$-purified nitroreductase proteins

3.3.3 Screening of YcnD_Bs SSM library for activity with S33 ................. 129

3.3.3.1 IC $I_{50}$ analysis of YcnD_Bs SSM library variants with S33.....................129

3.3.3.2 Active site modelling for selected YcnD Y227 mutants ........................131

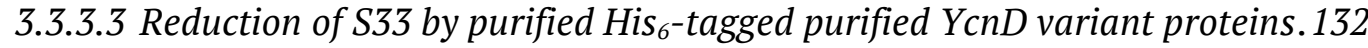

3.3.4 SOSR2 NfsA_Ec SDM library screening for S33 activity .................. 133

3.3.4.1 Screening of NfSA_Ec SDM library using the LacZ SOS assay................133

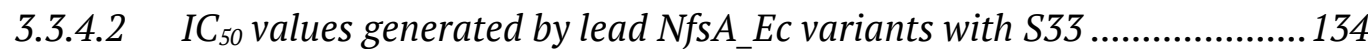


3.3.4.3 Active site modelling for selected NfSA_Ec SDM library mutants.

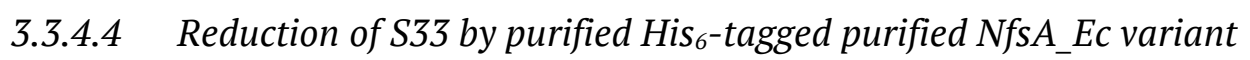
proteins

3.4 Discussion 138

3.4.1 Purified protein kinetics 138

3.4.2 Evaluation of wild-type candidate nitroreductase enzymes . 139

3.4.3 Screening of site-targeted nitroreductase libraries 139

3.4.4 High vs low-expressing pUCX plasmids 140

3.4.5 Alternative methods for screening nitroreductase variant libraries 141

Chapter 4. Directed evolution of YcnD_Bs and YfkO_Bs for improved S33 activation via random mutagenesis

4.1 Introduction.

4.1.1 Directed evolution of enzymes via random mutagenesis 144

4.1.2 GFP SOS assay and fluorescence-activated cell sorting 145

4.1.3 Previous FACS selection of a YfkO_Bs library ................................ 147

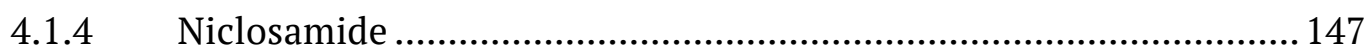

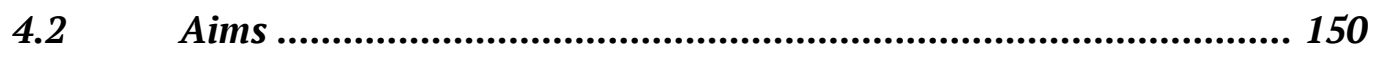

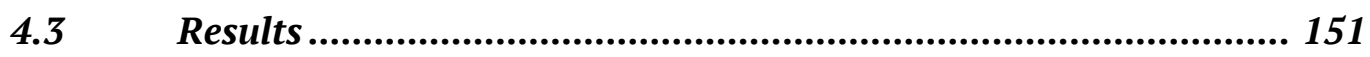

4.3.1 Evaluation of nitroreductase positive selection compounds ........... 151

4.3.2 YcnD_Bs and YfkO_Bs Round 1 error-prone library generation and screening

4.3.2.1 Creation of libraries and effects of niclosamide selection . 152

4.3.2.2 Fluorescence-activated cell sorting (FACS) of the YcnD_Bs and YfkO_Bs M1 libraries 154

\subsubsection{Screening of FACS-selected YcnD and YfkO M1 libraries using the GFP} SOS assay 
4.3.2.4 Growth inhibition (IC $C_{50}$ ) assays with $Y c n D$ and $Y f k O M 1$ libraries and S33

4.3.3 YcnD_Bs and YfkO_Bs round 2 error-prone library creation and screening

4.3.3.1 Creation of libraries and effects of niclosamide selection 159

4.3.3.2 Fluorescence-activated cell sorting (FACS) of YcnD_Bs and YfkO_Bs M2 libraries 160

4.3.3.3 Screening of FACS-sorted YcnD and YfkO M2 libraries using the GFP SOS assay 163

4.3.3.4 Growth inhibition (IC ${ }_{50}$ ) assays with $Y c n D$ and $Y f k O M 2$ libraries and S33 165

4.3.3.1 Activity of YfkO M2 variants with next-generation GDEPT prodrugs and PET imaging probes 167

4.3.3.2 Reduction of S33 by purified His $_{6}$-tagged purified $Y_{\text {fkO_Bs variant }}$ proteins

4.3.4 Effects of pANO-Duet-1:GFP plasmid on sensitising E. coli to nitroreductase-induced growth inhibition 173

4.3.4.1 Screening of GFP plasmid-containing or lacking E. coli strains with SOSinducing or non-inducing nitroaromatic compounds. 173

4.3.4.2 Growth inhibition (IC $C_{50}$ ) assays with pUCX:NfSA_Ec in 7NT or SOS-R4 cells and metronidazole.

4.3.4.3 Growth inhibition $\left(I C_{50}\right)$ assays with $p U C X: N f_{S} A_{-} E c$ in $7 N T$ or SOS-R4 cells and $H X 4$

4.3.5 Attempts to develop unstable GFP reporter strains ...................... 178

4.3.5.1 Cloning of unstable GFP variants into pANODuet -1 ..........................179

4.3.5.2 Growth inhibition (IC $C_{50}$ ) assays with pUCX:NfsA_Ec and GFP unstable variants in $7 N T$ with metronidazole 180

4.4 Discussion 
Chapter 5. Validation of positive selection compounds to improve NfsA_Ec activation of S33

5.1 Introduction.

5.1.1 The NfsA_Ec 7SM library

5.1.2 Chloramphenicol as a positive selection compound 190

5.1.3 Counter-screening of selected NfsA_Ec variants with nitroaromatic compounds

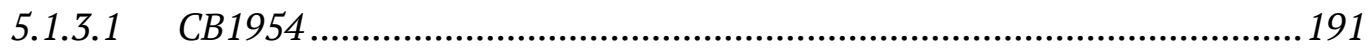

5.1.3.2 Metronidazole and zebrafish targeted cell ablation............................191

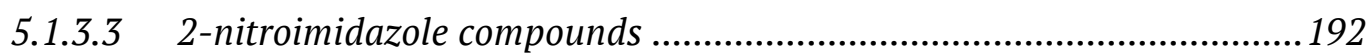

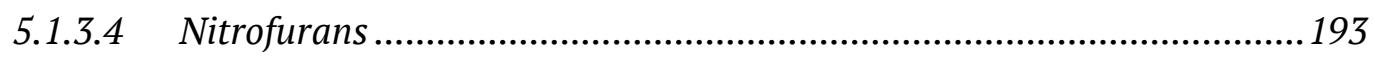

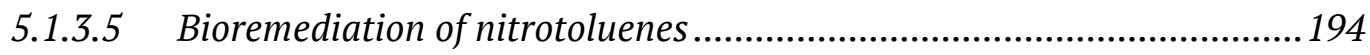

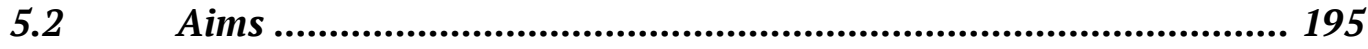

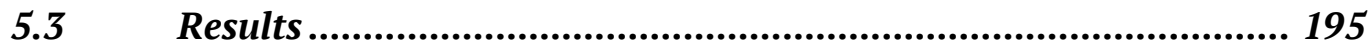

5.3.1 Evaluation of metronidazole and azomycin as surrogate compounds

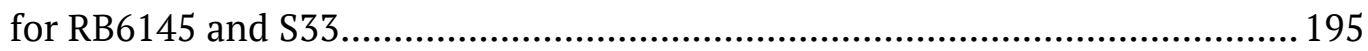

5.3.1.1 Correlation of S33 and metronidazole growth inhibition data ..............196

5.3.1.2 Correlation of RB6145 and azomycin growth inhibition ......................197

5.3.2 Effects of niclosamide selection on 7NT cells over-expressing NfsA_Ec

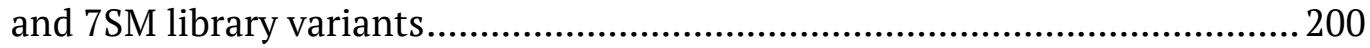

5.3.2.1 Niclosamide selection of 7NT cells over-expressing wild-type Nfs $A_{-}$Ec in low-expressing $p U C X$

5.3.2.2 Niclosamide selection of 7NT cells over-expressing NfSA_Ec 7SM library variants 201

5.3.2.3 Effects of niclosamide selection on activity of NfSA_Ec 7SM variants with other nitroaromatic compounds 
5.3.2.4 Heatmap and sequencing of $5 \mu M$ niclosamide-selected Nfs A_Ec 7SM variants .207

5.3.3 Counter-screen of improved metronidazole-activating NfsA_Ec 7SM library variants with S33..... 210

5.3.3.1 Growth inhibition $\left(I C_{50}\right)$ assays with $N f_{S} A_{-} E c$ C 7SM library variants with metronidazole and S33.. 210

5.3.3.2 Active site modelling for NfsA_Ec 7SM variants 5_4 and 5_6........ .214

5.3.3.3 Reduction of S33 by purified His ${ }_{6}$-tagged purified NfsA_Ec 7SM library variant proteins

5.3.4 Evaluation of chloramphenicol as a positive selection tool for active nitroreductases.

5.3.4.1 Chloramphenicol selection of the 58 nitroreductase library.......

5.3.4.2 Chloramphenicol selection of 7NT cells over-expressing NfSA_Ec 7SM library variants

5.3.4.3 Effects of chloramphenicol selection on activity of $N f S A_{-} E c$ C $7 S M$ variants with other nitroaromatic compounds 219

5.3.4.4 Heatmap and sequencing of $1 \mu \mathrm{g} / \mathrm{mL}$ chloramphenicol-selected $N f_{S} A_{-} E c$ $7 S M$ variants

5.3.5 Effects of niclosamide or chloramphenicol selection on amino acid distribution in 7SM library variants

5.3.5.1 Effects of $5 \mu M$ niclosamide or $1 \mu \mathrm{g} / \mathrm{mL}$ chloramphenicol selection on amino acid distribution in 7SM library variants

5.3.5.2 Changes in amino acid distribution of leading $5 \mu M$ niclosamide variants....

5.3.5.3 Changes in amino acid distribution of the leading chloramphenicolselected variants .229

5.4

Discussion 


\section{Chapter 6. Discovery of novel nitroreductases from multi-genomic}

DNA

6.1 Introduction

6.1.1 Creation and screening of metagenomic libraries 238

6.1.1.1 Construction of metagenomic DNA libraries 238

6.1.2 Methods for screening of metagenomic libraries.......................... 238

6.1.2.1 Expressing metagenomic genes using vector promoters

6.1.3 Previous attempts by the Ackerley lab to recover novel nitroreductase genes

6.1.3.1 Functional screening with niclosamide and coloured compounds..... 242

6.2

Aims

6.3 Results

6.3.1 Evaluation of the activity of members of the 58 nitroreductase library with nitro-masked chromophore compounds 244

6.3.1.1 Growth inhibition $\left(I C_{50}\right)$ assays with nitroreductases and nitro-masked chromophore compounds

\subsubsection{Correlation between 4-nitroimidazole or 5-nitroimidazole compound-} mediated growth inhibition

6.3.2 Attempts to create a metagenomic library................................... 248

6.3.2.1 Novel methods for cloning of metagenomic DNA libraries....................248

6.3.3 Creation of multi-genomic DNA library in pET28a+ 251

6.3.3.1 Selection of bacterial genomes with FatI-cleavable nitroreductases .....251

6.3.3.2 Screening of control nitroreductases with 4-nitroimidazole and 2-methyl4-nitroimidazole

6.3.3.3 Creation of multi-genomic DNA library .............................................254

6.3.3.4 Screening of multi-genomic library with niclosamide 255 
6.3.3.5 Counter-screening of niclosamide-selected colonies with 4-nitroimidazole and 2-methyl-4-nitroimidazole .257

6.3.3.6 Expression levels of nitroreductase hits from multi-genomic library.....260

6.4

Discussion 264

Chapter 7. Key findings, conclusions and future directions .............267

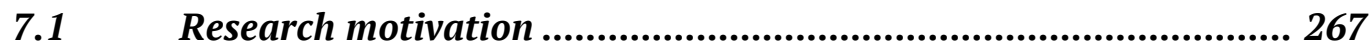

7.2 Summary of key findings ......................................................... 268

7.2.1 Discovery of wild-type nitroreductases that exhibited activity with S33

7.2.2 Screening site-targeted libraries for variants exhibiting improved activity with $\mathrm{S33}$

7.2.3 Creation and screening of random mutagenesis libraries for improved S33 activation 269

7.2.4 Use of nitroreductase positive selection compounds for the discovery of improved S33-activating variants 271

7.2.5 Utilising nitroaromatic selection and screening compounds for discovery of new nitroreductase enzymes 271

7.3 Critical evaluations of methods used in this research.................. 272

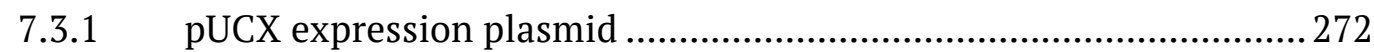

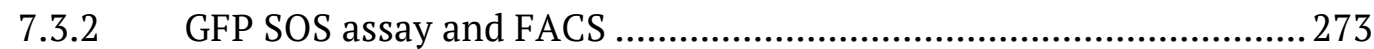

7.3.3 Enzyme evolution and protein fitness ......................................... 274

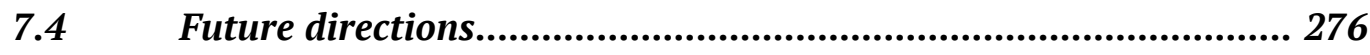


xvii

\section{Figures}

Figure 1-1 Schematic showing principle of gene directed enzyme prodrug therapy.

Figure 1-2 Mechanism of activation and genotoxicity of CB1954.

Figure 1-3 Schematic of type II (oxygen-sensitive; blue) versus type I (oxygen-independent; green) reduction of the nitroaromatic ring. 42

Figure 1-4 Chemical structures of prodrugs CB1954, SN23862, and PR104A.

Figure 1-5 Chemical structure of the nitrogen mustard prodrug SN34507... 50

Figure 1-6 Chemical structure of the 5-nitroimidazole prodrug

metronidazole.

Figure 1-7 Schematic showing the nuclear imaging of a reporter gene/probe following activation by a nitroreductase.

Figure 1-8 Chemical structures of ${ }^{18} \mathrm{~F} 2$-nitroimidazole hypoxia imaging agents F-MISO, EF5 and HX4.

Figure 1-9 In vivo binding of EF5 in HCT-116:nitroreductase tumour xenografts.

Figure 2-1 Plasmid map of nitroreductase expression vector pUCX:AzoR_Ec (high expression).

Figure 2-2 Plasmid map of nitroreductase expression vector pUCX (high expression).

Figure 2-3 Multiple-cloning site of nitroreductase expression vector pUCX (low expression).

Figure 2-4 Multiple-cloning site of nitroreductase expression vector pUCX (high expression).

Figure 2-5 Plasmid map of SOS-inducible GFP plasmid pANODuet-1:GFP. 68 Figure 2-6 Plasmid map of expression vector pET28a+, used for $\mathrm{His}_{6}$-tag protein expression and expression of multi-genomic DNA in this study. 
Xviii

Figure 2-7 Multiple-cloning site of expression vector pET28a+. 70

Figure 2-8 Phylogenetic tree of enzymes in the expanded nitroreductase candidate library. 84

Figure 2-9 Example of primers used to generate site targeted mutants. 86

Figure 2-10 Schematic of overlap PCR for generation of site directed mutants. 87

Figure 3-1 Chemical structures of the 5-nitroimidazole PET imaging-capable probe S33. 103

Figure 3-2 Hypoxia dependent binding of A) EF5 and B) S33 in a mouse HCT116 tumour xenograft model 105

Figure 3-3 Flow cytometry analysis of wild-type HCT-116 cells or HCT-116 cells stably expressing E. coli NfsA after in vitro exposure to EF5 or S33. . 106 Figure 3-4 Schematic showing the LacZ SOS assay in E. coli strain SOS$\mathrm{R} 2$ 109

Figure 3-5 Schematic showing the GFP SOS assay in E. coli strain SOSR4. 110

Figure 3-6 Western blot of nitroreductases stably expressed in HCT-116 cell line.

Figure 3-7 Sequence alignment of pUCX:AzoR_Ec and empty pUCX. 116

Figure 3-8 Plasmid map and mutations present in expression vector pUCX (low-expressing). 116

Figure 3-9 SDS-PAGE gel showing low- and high-expressing pUCX:YcnD_Bs relative protein expression levels from E. coli 7NT cells. 117

Figure 3-10 Correlation between metronidazole $\mathrm{IC}_{50}$ values of lowexpressing and high-expressing YcnD_Bs at IPTG concentrations ranging from $0 \mu \mathrm{M}$ to $50 \mu \mathrm{M}$. 120 
Figure 3-11 S33 mediated induction of the GFP SOS response in E. coli SOS$\mathrm{R} 4$ nitroreductase over-expression strains, challenged with either $2.5 \mu \mathrm{M}$ or $5 \mu \mathrm{M} \mathrm{S33.}$

Figure 3-12 Linear-logarithmic correlation between GFP SOS response induced by S33 in SOS-R4 nitroreductase over-expression strains and $\mathrm{IC}_{50}$ growth inhibitory effects of S33 in 7NT nitroreductase over-expression strains.

Figure 3-13 Mechanism of action of glucose oxidase-catalase oxygen scavenging system.

Figure 3-14 Kinetic parameters for the reduction of CB1954 by purified (His 6 -tagged) NfsA_Ec.

Figure 3-15 Modelling of active site of YcnD_Bs and three site-targeted variants.

Figure 3-16 Second-tier screening of S33 mediated induction of LacZ SOS response in E. coli SOS-R2 nitroreductase over-expressing strains challenged with $2.5 \mu \mathrm{M}$ S33. 133

Figure 3-17 Modelling of active site of NfsA_Ec and SDM library variants. 135 Figure 4-1 Schematic showing fluorescence-activated cell sorting (FACS) of E. coli SOS-R4 cells over-expressing inactive or active nitroreductases. ... 146 Figure 4-2 Chemical structure of niclosamide.

Figure 4-3 Growth inhibition of isogenic tolC- and tolC+ E. coli strains in response to increasing niclosamide challenge.

Figure 4-4 Growth inhibition of tolC- E. coli strain bearing pUCX-expressing nitroreductases in response to increasing niclosamide challenge. 150

Figure 4-5 Growth of 7NT nitroreductase over-expression strains, postchallenge with $0.5 \mu \mathrm{M}$ niclosamide. Figure 4-6 S33 mediated induction of fluorescence in E. coli SOS-R4 strains over-expressing either native YfkO, native YcnD, or the YfkO or YcnD M1 
libraries pre- or post- selection with $0.5 \mu \mathrm{M}$ niclosamide, challenged with 2.5 $\mu \mathrm{M}$ or $5 \mu \mathrm{M}$ S33.

Figure 4-7 Fluorescence of individual cells of wild-type YcnD_Bs and YfkO_Bs, and YcnD M1 and YfkO M1 libraries in SOS-R4 as observed in a FACS Vantage SE-Diva flow cytometer, following challenge with S33...... 155 Figure 4-8 S33 mediated induction of fluorescence in E. coli SOS-R4 strains over-expressing either native YcnD, empty pUCX or YcnD M1 libraries postFACS, challenged with $2.5 \mu \mathrm{M}$ S33. 156

Figure 4-9 S33 mediated induction of fluorescence in E. coli SOS-R4 strains over-expressing either native YfkO, empty pUCX, or the YfkO M1 library post FACS, challenged with $2.5 \mu \mathrm{M}$ S33. 157

Figure 4-10 S33 mediated induction of the GFP SOS response in E. coli SOSR4 YcnD and YfkO library nitroreductase over-expression strains. 161

Figure 4-11 Second-tier screening of S33 mediated induction of fluorescence in E. coli SOS-R4 cells over-expressing either native YcnD, lead YcnD M1 library variants, empty pUCX or YcnD M2 libraries post-FACS, challenged with $1.25 \mu \mathrm{M} \mathrm{S33.}$ 164

Figure 4-12 Second-tier screening of S33 mediated induction of fluorescence in E. coli SOS-R4 cells over-expressing either native YfkO, lead YfkO M1 library variants, empty pUCX or YfkO M2 libraries post-FACS, challenged with $2.5 \mu \mathrm{M}$ S33. 164

Figure 4-13 Chemical structures of the prodrug SN36506. 168

Figure 4-14 S33 mediated induction of fluorescence in E. coli SOS-R4 nitroreductase over-expression strains, challenged with $150 \mu \mathrm{M}$ SN36506. 169

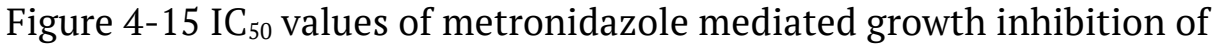
NfsA_Ec in 7NT cells with or without the pANODuet-1:GFP plasmid 
Figure 4-16 Linear correlation between background fluorescence levels and $\mathrm{IC}_{50}$ growth inhibitory effects of metronidazole on SOS-R4 cells overexpressing pUCX:NfsA_Ec.

Figure 4-17 IC 50 values for HX4 mediated growth inhibition of NfsA_Ec overexpressed in biological replicates of 7NT or SOS-R4 strains.

Figure 4-18 Sequence alignment of GFPmut3b and unstable GFP variant genes (including amino acid translations).

Figure 4-19 Metronidazole mediated growth inhibition of 7NT cells coexpressing pUCX:NfsA_Ec and pANODuet-1:GFP variants.

Figure 5-1 Active site residues of NfsA_Ec targeted for site saturation mutagenesis in the 7SM library.

Figure 5-2 Chemical structure of chloramphenicol.

Figure 5-3 Chemical structure of the 5-nitroimidazole compounds metronidazole and S33.

Figure 5-4 Chemical structures of 2-nitroimidazole compounds RB6145, RSU1069, and azomycin.

Figure 5-5 Chemical structures of the nitrofuran drugs nitrofurantoin and nitrofurazone.

Figure 5-6 Chemical structures of TNT (2,4,6-trinitrotoluene) and 2,4-DNT (2,4-dinitrotoluene).

Figure 5-7 Log-log correlation between $\mathrm{IC}_{50}$ growth inhibitory effects of S33 and metronidazole in 7NT nitroreductase over-expression strains. 197

Figure 5-8 Log-log correlation between $\mathrm{IC}_{50}$ growth inhibitory effects of RB6145 and azomycin in 7NT nitroreductase over-expression strains 200 Figure 5-9 SDS-PAGE showing relative protein expression levels of NfsA_Ec 7SM library variants and NfsA_Ec in low-expressing pUCX in the E. coli 7NT strain. 
Figure 5-10 Nitroaromatic compound mediated growth inhibition of NfsA_Ec 7SM library variants in 7NT.

Figure 5-11 Log-log correlation between $\mathrm{IC}_{50}$ growth inhibitory effects of S33 and metronidazole in 7NT NfsA_Ec over-expression strains.

Figure 5-12 Modelling of active site of NfsA_Ec and 7SM library variants. 214 Figure 5-13 Kinetic parameters for the reduction of S33 by purified (His $6^{-}$ tagged) NfsA_Ec 7SM library variants 5_4 and 5_6 216

Figure 5-14 Growth of 7NT nitroreductase over-expression strains, postchallenge with chloramphenicol.

Figure 5-15 Nitroaromatic compound mediated growth inhibition of 7NT cells over-expressing NfsA_Ec 7SM library variants. 221

Figure 6-1 Different modes of gene expression utilising a vector $\mathrm{T} 7$ promoter in a heterologous bacterial host. 240

Figure 6-2 Chemical structures of the annular isomers of 4(5)nitroimidazole

Figure 6-3 Chemical structures of the annular isomers of 2-methyl-4(5)nitroimidazole

Figure 6-4 Log-log correlation between $\mathrm{IC}_{50}$ growth inhibitory effects of 2methyl-4-nitroimidazole and S33 in 7NT nitroreductase over-expression strains.

Figure 6-5 Log-log correlation between $\mathrm{IC}_{50}$ growth inhibitory effects of 2methyl-4-nitroimidazole and S33 in 7NT nitroreductase over-expression strains.

Figure 6-6 Log-log correlation between IC $_{50}$ growth inhibitory effects of 4nitroimidazole and 2-methyl-4-nitroimidazole in 7NT nitroreductase overexpression strains. 248

Figure 6-7 FatI and NcoI restriction enzyme DNA cleavage sites. 249 
xxiii

Figure 6-8 Modified pET28a+ multiple-cloning site for expression of metagenomic fragments

Figure 6-9 Partial restriction enzyme digest of multi-genomic DNA with FatI.

Figure 6-10 Activation of nitro-masked chromophores by multi-genomic library variants also capable of detoxifying $0.5 \mu \mathrm{M}$ niclosamide. 258

Figure 6-11 SDS-PAGE gel showing relative protein expression levels of His $_{6}$-tagged nitroreductases and nitroreductase hits from multi-genomic library in $7 \mathrm{NT}$. 
xxiv 


\section{Tables}

Table 2-1 Primers used in this study 60

Table 2-2 Escherichia coli strains used in this study 62

Table 2-3 Bacterial strains used as sources of genomic DNA in this study... 62

Table 2-4 Plasmids used for cloning in this study 63

Table 2-5 Liquid media recipes 71

Table 2-6 Antibiotic final concentrations 72

Table 2-7 PCR protocol for Biomix Red 73

Table 2-8 PCR components and protocol for Phusion ${ }^{\mathrm{TM}}$ 74

Table 2-9 PCR components and thermocycling parameters for GeneMorph ${ }^{\circledR}$ II

Table 2-10 Ligation protocol with T4 DNA ligase. 76

Table 2-11 TSS Buffer recipe 77

Table 2-12 Oxidoreductase enzymes in the expanded nitroreductase candidate library

Table 2-13 His-tag purification buffer recipes 91

Table 2-14 SDS-PAGE gel recipes ......................................................... 96

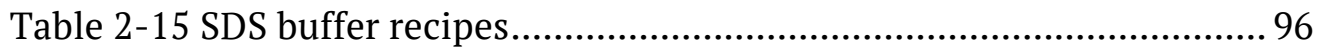

Table 2-16 Recipes of buffers required for measurement of $\beta$-galactosidase activity in LacZ SOS assays.

Table 3-1 IC 50 values of S33 mediated growth inhibition of lead variants from SOS-R2 YcnD_Bs SSM library in pUCX:KG challenged with up to $70 \mu \mathrm{M}$ S33.

Table 3-2 Plasmid mutations differentiating pUCX (high-expressing) and pUCX (low-expressing). 
xxvi

Table 3-3 IC 50 values of S33 mediated growth inhibition of 7NT bearing highexpressing or low-expressing YcnD_Bs challenged with up to $200 \mu \mathrm{M}$ metronidazole.

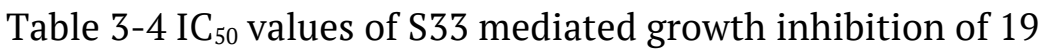

nitroreductases from the 7NT E. coli 58 nitroreductase over-expression

library.

Table 3-5 Michaelis-Menten kinetic parameters for NfsA_Ec and CB1954. 127

Table 3-6 Michaelis-Menten kinetic parameters estimated for nitroreductases and S33.

Table 3-7 IC 50 values for S33 mediated growth inhibition of lead variants of

YcnD_Bs in E. coli 7NT challenged with up to $100 \mu \mathrm{M}$ S33. 130

Table 3-8 Michaelis-Menten kinetic parameters for YcnD variants and S33.

Table 3-9 IC 50 values of S33 mediated growth inhibition of lead variants from SOS-R2 NfsA_Ec SDM library challenged with up to $100 \mu \mathrm{M}$ S33. 134

Table 3-10 Michaelis-Menten kinetic parameters for NfsA_Ec mutants and S33. 137

Table 4-1 IC 50 values of S33 mediated growth inhibition of YcnD M1 variants 158

Table 4-2 IC 50 $_{0}$ values of S33 mediated growth inhibition of YfkO M1 variants 159

Table 4-3 IC 50 values for S33 mediated growth inhibition of YcnD M1 and M2 variants 165

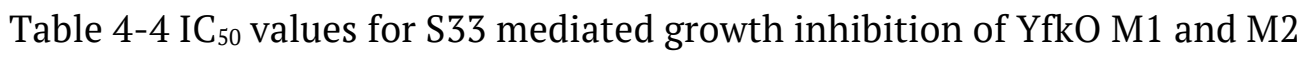
variants 166

Table 4-5 IC S0 $_{50}$ values of prodrug or imaging probe mediated growth inhibition of candidate nitroreductases. 
xxvii

Table 4-6 Michaelis-Menten kinetic parameters for YfkO M2 variants and S33.

Table 5-1 NfsA_Ec amino acid residues targeted for site saturation mutagenesis in the7SM library with degenerate codons.

Table 5-2 IC $_{50}$ values for S33 or metronidazole mediated growth inhibition of 18 highly active nitroreductases from the 7NT E. coli 58 nitroreductase overexpression library. 196

Table 5-3 IC 50 $_{5}$ values for RB6145 or azomycin mediated growth inhibition of 18 nitroreductases from the 7NT E. coli nitroreductase over-expression library. 199

Table 5-4 Average nitroaromatic compound mediated growth inhibition of NfsA_Ec 7SM library variants in 7NT. 206

Table 5-5 Sequencing results and percentage growth inhibition of $5 \mu \mathrm{M}$ niclosamide-selected NfsA_Ec 7SM variants with six different nitroaromatic compounds. 209

Table 5-6 IC 50 values for S33 or metronidazole mediated growth inhibition of E. coli 7NT cells bearing lead variants from the NfsA_Ec 7SM library. 212

Table 5-7 Michaelis-Menten kinetic parameters estimated for NfsA_Ec 7SM library variants.

Table 5-8 Average nitroaromatic compound mediated growth inhibition of NfsA_Ec 7SM library variants in 7NT.

Table 5-9 Sequencing results and percentage growth inhibition of $1 \mu \mathrm{g} / \mathrm{mL}$ chloramphenicol-selected NfsA_Ec 7SM variants with six different nitroaromatic compounds.

Table 5-10 Distribution of amino acids across niclosamide or chloramphenicol-selected NfsA_Ec 7SM variants. 225

Table 5-11 Changes in amino acid distribution of $5 \mu \mathrm{M}$ niclosamide-selected 7SM variants exhibiting improved activity with other nitroaromatic compounds. 228 
xxviii

Table 5-12 Changes in amino acid distribution of $1 \mu \mathrm{g} / \mathrm{mL}$ chloramphenicolselected 7SM variants exhibiting improved activity with other nitroaromatic compounds. 230

Table 6-1 IC 50 $_{50}$ values of 4-nitroimidazole or 2-methyl-4-nitroimidazole mediated growth inhibition of 18 nitroreductases from the 7NT E. coli 58 nitroreductase over-expression library.

Table 6-2 List of candidate nitroreductases containing a FatI restriction site immediately preceding the ATG start codon

Table 6-3 Activity of control nitroreductases in pET28a+ with niclosamide and nitro-masked chromophores.

Table 6-4 Sequencing results of metagenomic hits that could detoxify $0.5 \mu \mathrm{M}$ niclosamide.

256

Table 6-5 Sequencing results of $0.5 \mu \mathrm{M}$ niclosamide-selected metagenomic hits that could reduce either 4-nitroimidazole or 2-methyl-4nitroimidazole. 259 


\section{List of abbreviations and definitions}

\begin{tabular}{|c|c|}
\hline 2,4-DNT & 2,4-dinitrotoluene \\
\hline $5-\mathrm{FU}$ & 5-fluorouracil \\
\hline aa & Amino acid \\
\hline ACSRC & Auckland Cancer Society Research Centre \\
\hline AKR1C3 & Aldo-keto reductase $1 \mathrm{C} 3$ \\
\hline Amp & Ampicillin \\
\hline BAC & Bacterial artificial chromosome \\
\hline CD & Cytosine deaminase \\
\hline cds & Coding DNA sequence \\
\hline CDEPT & Clostridium-directed enzyme prodrug therapy \\
\hline CMV & Cytomegalovirus \\
\hline CTL & Cytotoxic T lymphocytes \\
\hline $\mathrm{dd}_{2} \mathrm{O}$ & Double-distilled $\mathrm{H}_{2} \mathrm{O}$ \\
\hline $\mathrm{diH}_{2} \mathrm{O}$ & Deionised $\mathrm{H}_{2} \mathrm{O}$ \\
\hline DMSO & Dimethyl sulfoxide \\
\hline epPCR & Error-prone polymerase chain reaction \\
\hline FACS & Fluorescence-activated cell sorting \\
\hline F-MISO & Fluoro-misonidazole \\
\hline GCV-TP & Ganciclovir triphosphate \\
\hline GFP & Green fluorescent protein \\
\hline GDEPT & Gene-directed enzyme prodrug therapy \\
\hline gDNA & Genomic DNA \\
\hline $\mathrm{Gm}$ & Gentamicin \\
\hline GM-CSF & Granulocyte-macrophage colony-stimulating factor \\
\hline $\mathrm{HCC}$ & Hepatocellular carcinoma \\
\hline HSV-TK & Herpes simplex virus-thymidine kinase \\
\hline IPTG & Isopropyl $\beta$-D-1-thiogalactopyranoside \\
\hline Kan & Kanamycin \\
\hline MCS & Multiple-cloning site \\
\hline MRI & Magnetic resonance imaging \\
\hline MTD & Maximum tolerated dose \\
\hline NMIBC & Non-muscle invasive bladder cancer \\
\hline PCR & Polymerase chain reaction \\
\hline PET & Positron emission tomography \\
\hline $\mathrm{Rb}$ & Retinoblastoma tumour suppressor protein \\
\hline rbs & Ribosome binding site \\
\hline Spec & Spectinomycin \\
\hline SSTR2 & Somatostatin receptor subtype 2 \\
\hline Tet & Tetracycline \\
\hline T-Vec & Talimogene laherparepvec \\
\hline
\end{tabular}


XXX 


\section{Chapter 1. Introduction}

\subsection{Targeted novel anti-cancer therapeutics}

The most recent statistics from the Ministry of Health show the continuing burden of cancer in our aging population, ending another year as the leading cause of death in New Zealand (Ministry of Health, 2016). Despite improvements in chemotherapy and radiotherapy over the last century, offtarget side effects still persist (Bernier et al., 2004; Chabner and Roberts, 2005). Furthermore, the limitations of treatment in hypoxic tumours, as well as the persistence of treatment-resistant cancer cells, make it clear that the development of more targeted therapies are necessary (Barker et al., 2015; Hanahan and Weinberg, 2011; Holohan et al., 2013).

One method of targeted treatment, cancer immunotherapy, seeks to utilise the patient's own immune system to detect and destroy cancerous cells (Farkona et al., 2016; de Visser et al., 2006). In 2011 the FDA granted approval for the use of ipilimumab, a monoclonal antibody, as a treatment for metastatic melanoma patients (Farkona et al., 2016; Mellman et al., 2011). The development of this treatment, which targets the over-expressed immune system down-regulatory protein CTLA-4, earned researcher James P. Allison the prestigious Wolf Prize in Medicine, to be presented later this year. Some other immunotherapy strategies, such as cancer vaccines and immune checkpoint inhibitors, have also shown promise in clinical trials (Farkona et al., 2016).

Alternatively, a form of targeted chemotherapy that has reached Phase III trials involves the use of hypoxia-activated prodrugs, which allows for the administration of chemically inert compounds that become potent cellkilling toxins upon activation solely in anoxic regions, i.e. in hypoxic cores of tumours (Hunter et al., 2016; Wilson and Hay, 2011).

Another type of targeted cancer therapy involves the administration of exogenous agents, such as viruses and bacteria, which possess the ability to target and kill tumour cells. 


\subsubsection{Oncolytic viruses as targeted anti-cancer therapeutics}

Oncolytic viruses exhibit selective targeting of cancerous cells over normal tissue, in part due to their ability to exploit phenotypic changes in these cells that occur upon malignant transformation (Seymour and Fisher, 2016). The ability of oncolytic viruses to both selectively destroy cancerous cells and induce the host immune response make them an ideal candidate for synergistic therapy with cancer immunotherapies, which could assuage the tumour microenvironment's suppression of this response (Chiocca and Rabkin, 2014; Slaney and Darcy, 2015). Oncolytic viruses can be divided into two main types: viruses that are non-virulent in humans but replicate preferentially in cancer cells, such as reovirus; and viruses that naturally infect human cells but have been further genetically engineered to be cancer-specific, such as adenovirus and herpes simplex virus (Chiocca, 2002; Chiocca and Rabkin, 2014; Everts and van der Poel, 2005; Fukuhara et al., 2016; Russell et al., 2012).

In 2005 the E1B deleted adenovirus H101 was approved for treatment of advanced nasopharyngeal carcinoma in combination with chemotherapy in China following Phase III clinical trials showing improved responses in patients over chemotherapy alone, making it the first approved oncolytic viral cancer treatment on the market (Garber, 2006; Yu and Fang, 2007). Following this success, there has been renewed pharmaceutical interest in the use of oncolytic virotherapy to combat cancer, with recent clinical trials showing promising results (Fukuhara et al., 2016; Pol et al., 2016; Seymour and Fisher, 2016). However, an ongoing limitation is the inability to successfully achieve systemic spread of the therapy beyond local intratumoural injection, which limits the efficacy of these treatments for treating hard-to-access and metastatic cancers (Parato et al., 2005; Russell et al., 2012). Despite this, some therapies have shown enough promise in clinical trials to be approved for patients as a last resort following failure of traditional cancer treatments, and a few of these will be described in the following sections. 


\subsubsection{Talimogene laherparepvec (T-Vec; or Imlygic ${ }^{\circledR}$, formerly Oncovex ${ }^{G M-C S F}$ )}

T-Vec is a modified HSV- 1 virus with deletions in the $\gamma 34.5$ and $\alpha 47$ genes and insertion of human granulocyte-macrophage colony-stimulating factor (GM-CSF). Collectively these modifications enhance its replication in cancer cells and the host anti-tumour response while rendering it unable to replicate in normal tissue (Chiocca, 2002; Fukuhara et al., 2016). T-Vec was evaluated in a Phase III trial for patients with stage IIIb/IV melanoma and the results from intralesional administration, when compared to GM-CSF administration alone, showed an improvement in median survival rate by four months, with $11 \%$ of patients going into remission (Andtbacka et al., 2015). This led T-Vec to be approved as a treatment for unresected melanoma in the USA in 2015 and Europe in 2016 (Fukuhara et al., 2016). TVec is currently undergoing Phase Ib/II trials in combination with ipilimumab to further stimulate the host immune response (Dolgin, 2015).

\subsubsection{2 $G 47 \Delta$}

The second-generation HSV-1 virus G207 was created through deletion of the ICP6 gene, which is involved in viral DNA synthesis, deletion of $\gamma 34.5$, and insertion of an E. coli lacZ gene in place of the latter (Mineta et al., 1995). G47 $\Delta$ was created from G207 through further deletion of the $\alpha 47$ gene to enhance MHC Class I presentation and viral replication (Fukuhara et al., 2016; Todo et al., 2001). Successful completion of a Phase I/IIa trial with recurrent glioblastoma conducted in Japan, showing good tolerance of intratumoral injection of the virus (Todo et al., 2014), was followed by an ongoing Phase II study in 2015 for recurrent glioblastoma patients, with injection of G47 $\Delta$ into the brain tumour (Fukuhara et al., 2016). G47 $\Delta$ was designated a 'Sakigake' breakthrough therapy drug by the Japanese government in February 2016, which would fast-track approval for its use following successful completion of the Phase II trial (Fukuhara et al., 2016). 


\subsubsection{JX-594 (Pexastimogene devacirepvec; Pexa-Vec)}

JX-594 is a modified vaccinia virus, with the thymidine kinase gene replaced by human GM-CSF and lacZ, allowing for cancer-selective replication and improvement of the anti-tumour immune response (Chiocca, 2002; Kim et al., 2006; Kirn and Thorne, 2009; Parato et al., 2012). A randomized Phase II trial in patients with advanced hepatocellular carcinoma (HCC) showed intratumoral injection of high-dose JX-594 improved survival by over seven months over a low-dose treatment (Heo et al., 2013). A Phase III trial with advanced HCC in 2015 is still ongoing (Fukuhara et al., 2016).

\subsubsection{CG0070}

CG0070 is an engineered A5 adenovirus containing a human E2F-1 promoter driving the $E 1 A$ gene, restricting viral replication to $\mathrm{Rb}$ (retinoblastoma tumour suppressor protein) mutated cells, which are commonly seen in bladder cancer (Chiocca, 2002; Ramesh et al., 2006). It also contains the GMCSF gene inserted behind a E1A-activated promoter, further stimulating the patient's immune response (Ramesh et al., 2006). Results from a randomised Phase II/III trial of 15 patients with non-muscle-invasive bladder cancer, where CG00070 was compared with other standard intravesical therapies, showed no significant difference in patient response (Fukuhara et al., 2016). However, patients presenting with $\mathrm{Rb}$-defective cancer exhibited a better durable response to CG00070 than patients with non-Rb-defective cancer (Fukuhara et al., 2016). A single-arm Phase III trial with patients with nonmuscle invasive bladder cancer (NMIBC) is currently ongoing (Dolgin, 2015).

\subsubsection{Reolysin $\AA$}

Reolysin is a replication-competent form of the T3D strain of reovirus, a double-stranded RNA virus that preferentially targets cells with an activated Ras signalling pathway (Gollamudi et al., 2010). A Phase II trial of metastatic melanoma patients showed Reolysin replication in melanoma tissue 
following intravenous administration of the treatment; however efficacy of the treatment was not established (Galanis et al., 2012). A Phase III trial comparing intravenously administered Reolysin in combination with the chemotherapeutics paclitaxel and carboplatin, vs. the latter two compounds on their own, was conducted on patients with recurrent and/or metastatic head and neck cancer, and improved overall survival was observed in patients without metastatic disease (Fukuhara et al., 2016). Reolysin was granted orphan drug approval by the FDA for treatment of malignant glioma, ovarian and pancreatic cancers in 2015 (Fukuhara et al., 2016).

\subsubsection{Bacteria as targeted anti-cancer therapeutics}

In the late $19^{\text {th }}$ century, physician William Coley developed 'Coley's toxins', a mixture of two heat-killed bacteria species, Streptococcus pyogenes and Serratia marcescens, which he administered to the tumour site of inoperable sarcoma patients (Felgner et al., 2016; Patyar et al., 2010). In many patients he observed fever, followed by tumour regression and occasionally complete disappearance of the tumour, which we now know was caused by stimulation of the host immune response (Felgner et al., 2016; Kucerova and Cervinkova, 2016).

In a different application of bacteria as anti-cancer therapeutics, some facultative and obligate anaerobe bacterial species possess a natural inclination for migration towards, growth, and proliferation in the anaerobic tumour microenvironment and in cancer cells (Felgner et al., 2016; Forbes, 2010; Lehouritis et al., 2013; Van Dessel et al., 2015).

\subsubsection{Salmonella}

Salmonella is a facultative anaerobe that exhibits chemotaxis to the tumour microenvironment (Kasinskas and Forbes, 2007). Due to the ability of Salmonella to colonise both aerobic and anaerobic tissue, they have the innate potential to accumulate in healthy organs such as the liver and spleen 
and must be engineered for tumour specificity (Felgner et al., 2016). For example, some strains of Salmonella have been made auxotrophic for factors found in abundance in the tumour microenvironment (Felgner et al., 2016). An attenuated strain of engineered Salmonella, VNP20009, showed rapid clearance from the blood to undetectable levels within 24 hours in both mice and primates, and accumulated in the immune-privileged tumour microenvironment at ratios of 1000:1 relative to accumulation in the liver in mice (Clairmont et al., 2000). Unfortunately the testing of VNP20009 as a sole cancer agent administered intravenously in a Phase I clinical trial showed limited tumour colonisation and no tumour regression (Toso et al., 2002).

\subsubsection{Listeria}

An attenuated form of the facultative anaerobe Listeria monocytogenes, CRS207, was administered to metastatic pancreatic cancer patients in a Phase II study alongside the cancer vaccine GVAX, the immunosuppressant cyclophosphamide, and a protein overproduced in certain cancers (mesothelin). These patients showed a 2.2 month improvement in overall survival over those receiving the control combination treatment (Le et al., 2015). However, a follow-up Phase II trial showed no improvement following sole CRS-207 treatment over standard chemotherapy, and future trials intend to combine the bacteria with immune checkpoint inhibitors (Maxmen, 2017).

\subsubsection{Clostridia}

Clostridium species, which are obligate anaerobes, can be injected into a patient as spores and will only germinate in hypoxic conditions i.e. within the solid core of a tumour (Kubiak and Minton, 2015; Lee, 2012; Lehouritis et al., 2013; Mowday et al., 2016a). One of the most currently promising Clostridium strains, C. novyi-NT, which lacks the gene coding for the 
neurotoxic $\alpha$-toxin, has shown promise following intratumoral injection of spores, with a $21 \%$ complete response in dogs, and a sole human patient with advanced leiomyosarcoma showing tumour colonization and shrinkage (Roberts et al., 2014). However in dogs treated by intravenous spore administration, stable disease was the best outcome achieved at doses having acceptable toxicity, which indicated that the limitation for the treatment is still the inability to achieve high levels of tumour colonisation following distal administration of spores (Krick et al., 2012). It is also acknowledged that the ability of Clostridium to only colonise anaerobic regions may limit its efficacy in smaller tumours that lack a dense hypoxic core (Felgner et al., 2016).

\subsection{Gene directed enzyme prodrug therapy}

There has been interest over the past few decades in using viral and bacterial vectors for their tumour-targeting and gene-delivery properties, giving rise to a form of targeted cancer treatment called gene-directed enzyme prodrug therapy (GDEPT) (Baban et al., 2010; Hermiston and Kuhn, 2002). In GDEPT, a foreign gene is delivered to cancer cells and the subsequently expressed enzyme converts a systemically administered, non-toxic prodrug into its cell-killing active form (Figure 1-1; Karjoo et al., 2016; Malekshah et al., 2016; Portsmouth et al., 2007; Zhang et al., 2015). Due to the inability of gene delivery vectors to transduce more than $10 \%$ of tumour cells (Hamstra et al., 1999), the activated drug should also be able to diffuse short distances to kill closely neighbouring cancer cells, which allows for greater damage to the tumour. This is commonly called the 'bystander effect' (Xu and McLeod, 2001). 


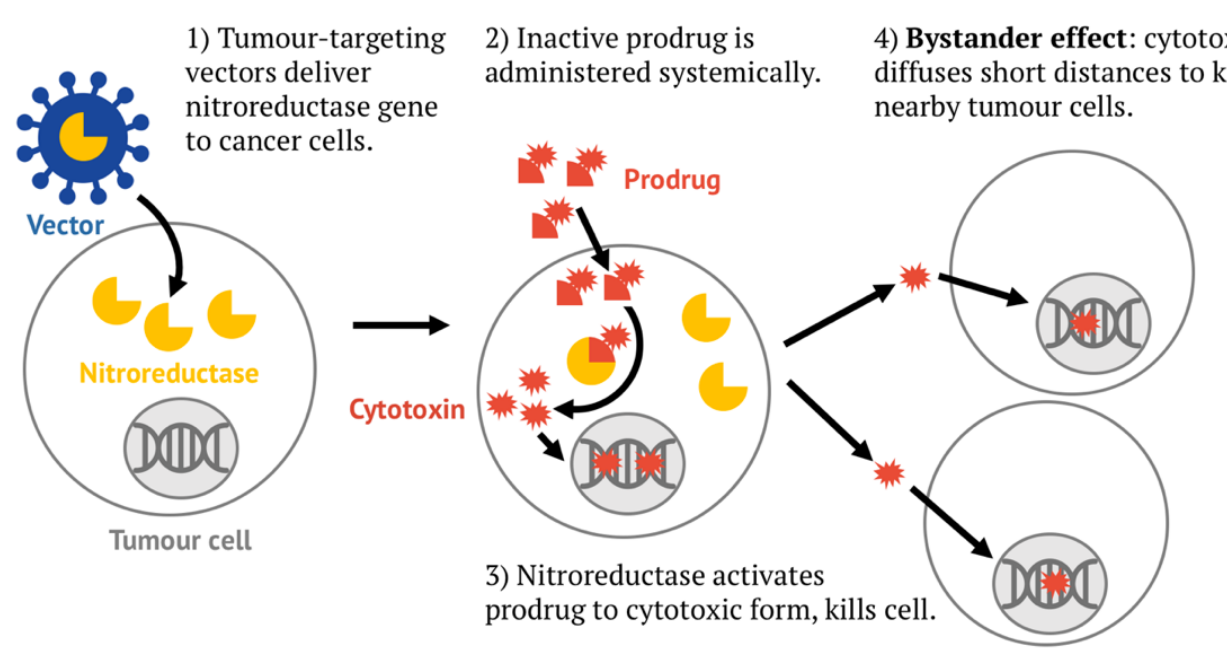

Figure 1-1 Schematic showing principle of gene directed enzyme prodrug therapy.

Many different enzyme/prodrug combinations have been investigated for GDEPT, with some advancing to clinical trials. Three of the most promising systems: herpes simplex virus-thymidine kinase (HSV-TK) and ganciclovir; cytosine deaminase and 5-fluorouracil; and nitroreductase and CB1954 will be discussed in the following sections.

\subsubsection{HSV-TK and ganciclovir}

One of the earliest GDEPT enzyme/prodrug pairings investigated involved the HSV-TK enzyme and the antiviral drug ganciclovir. HSV-thymidine kinase phosphorylates ganciclovir to ganciclovir monophosphate, which is then converted by endogenous human kinases into ganciclovir triphosphate (GCV-TP; Duarte et al., 2012; Zhang et al., 2015). GCV-TP competes with the nucleotide precursor dGTP for subsequent incorporation into DNA during replication, which leads to inhibition of DNA polymerase as well as single strand break formation in the replicated DNA strand, causing cellular apoptosis (Dachs et al., 2009). The bystander effect of the activated metabolites is mediated through cellular gap junctions (van Dillen et al., 2002). Clinical trials of this enzyme/prodrug combination as a sole treatment have shown limited success, with a Phase III trial in metastatic glioblastoma 
multiforme patients combining intratumoral injection of $h s v-t k$ encoding retroviral vector, ganciclovir and standard treatment (surgery and radiotherapy) showing no improvement in survival over standard treatment alone (Rainov, 2000). Problems encountered with this system include a lack of functioning gap junctions in many tumour tissues, and a requirement for a prohibitively high dose of ganciclovir to patients to compete with HSVTK's high affinity for its natural substrate, thymidine (Karjoo et al., 2016). Recently, alternative prodrugs such as valacyclovir have been paired with HSV-TK, and different clinical trials involving prostate cancer, leukemia and malignant glioma patients are currently underway using these new pairings (Zhang et al., 2015).

\subsubsection{Cytosine deaminase/5-fluorocytosine}

Bacterial or fungal cytosine deaminase enzymes can convert the prodrug 5fluorocytosine into the anticancer agent 5-fluorouracil (5-FU), which is then further converted by intracellular enzymes into metabolites that form 5FUDNA and 5FU-RNA and interfere with thymidine synthesis to cause cellular apoptosis (Karjoo et al., 2016). 5-FU also readily diffuses to neighbouring cells to cause a bystander effect (Karjoo et al., 2016). One of the main limitations of cytosine deaminase enzymes used in this combination therapy is their natural high preference for cytosine, and high doses of 5fluorocytosine must be administered to patients to allow for enough conversion to 5-FU to have a therapeutic effect (Karjoo et al., 2016). In a Phase I trial of recurrent glioma patients, administration of Toca 511 (codon-optimized yeast cytosine deaminase delivered by a retroviral vector via intratumoural injection) together with an extended-release form of 5fluorocytosine yielded a six-month increase in median overall survival (Cloughesy et al., 2016). The same treatment is currently undergoing Phase II/III trials in recurrent glioblastoma and anaplastic astrocytoma patients (Cloughesy et al., 2016). 


\subsubsection{Nitroreductases and CB1954}

Nearly half a century ago, the prodrug 5-(aziridin-1-yl)-2,4dinitrobenzamide (CB1954) was found to inhibit the growth of Walker 256 rat carcinoma (Cobb et al., 1969). Subsequent studies showed that this was due to high tumour expression of the enzyme DT diaphorase, a quinone reductase that promiscuously and selectively reduces the 4-nitro group of CB1954 to a hydroxylamine group, which is then further reduced by cellular enzymes to a toxic, DNA interstrand cross-linking agent (Figure 1-2; Knox et al., 1988a, 1991). However attempts to generate the same effect in human cancer lines fell short due to a 10 -fold lower efficiency $\left(k_{c a t} / K_{M}\right)$ of human DT diaphorase with the prodrug, largely due to a single amino acid difference between the two enzymes (Boland et al., 1991; Chen et al., 1997). It was discovered soon after that the $E$. coli $\mathrm{NfsB}$ nitroreductase enzyme was able to activate CB1954, with a 86-fold increase in $k_{\text {cat }} / K_{M}$ over rat DT diaphorase (Anlezark et al., 1992; Knox et al., 1992). E. coli NfsB reduces CB1954 at either the 4-nitro or the 2-nitro position in an approximately equimolar ratio (Figure 1-2; Knox et al., 1992), but downstream products of the 2hydroxyamine, as well as exerting similar cytotoxic effects in repaircompetent human tumour cell lines, also exhibit an improved bystander effect (Helsby et al., 2004).

A Phase I trial demonstrated that the recommended dosing for intravenous administration of CB1954 for the prodrug was $24 \mathrm{mg} / \mathrm{m}^{2}$, with an AUC of 5.8 $\mu \mathrm{M} / \mathrm{h}$ and a mean peak serum concentration of $6.3 \mu \mathrm{M}$ (Chung-Faye et al., 2001). A following Phase I trial demonstrated the safety of intratumoral administration of a replication-deficient adenovirus expressing nitroreductase (CTL102) to patients with secondary liver cancer (Palmer et al., 2004). A Phase I/II trial with localised prostate cancer patients utilising CTL102 and $24 \mathrm{mg} / \mathrm{m}^{2}$ CB1954 yielded similar blood levels of CB1954 (mean AUC of $6.3 \mu \mathrm{M} / \mathrm{h}$, mean peak serum concentration of $7.8 \mu \mathrm{M}$ ) and showed evidence of $n f s B$ expression in tumours across all dose levels of CTL102; however only limited evidence of anti-tumour activity was observed (Patel et al., 2009). The major factor limiting success of this human trial was likely 
due to the $K_{M}$ of $E$. coli NfsB with CB1954 being significantly greater than the maximum achievable serum concentrations - purified protein kinetics conducted with this pairing by multiple research groups indicate that the $K_{M}$ of NfsB for CB1954 may be as much as 500-fold higher than the achievable peak serum concentration of CB1954 in patients (Williams et al., 2015). Thus, improvements to this gene/prodrug pairing may be necessary for successful clinical trial outcomes. Potential strategies for this are discussed in Section 1.4.

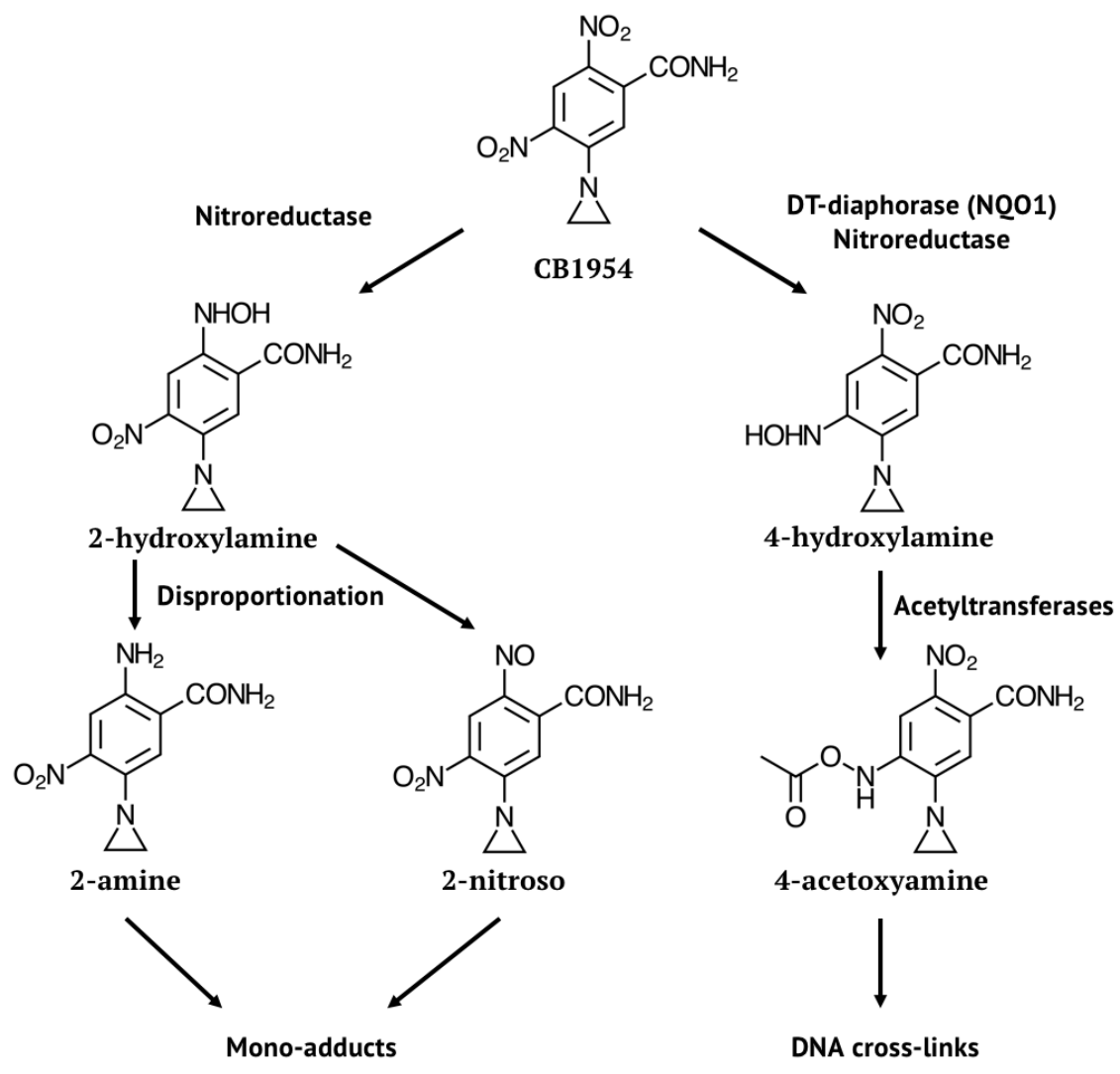

Figure 1-2 Mechanism of activation and genotoxicity of CB1954.

\subsection{Nitroreductase enzymes}

Nitroreductases are a widely distributed bacterial family of flavin-utilising, primarily homodimeric oxidoreductase enzymes that can utilise NADH or NADPH to reduce the nitro group on aromatic rings (Williams et al., 2015). The nitro group is a strongly electron-withdrawing group that, upon 
reduction to a hydroxylamine or amine group, is converted to a strong electron-donating moiety. When located on an aromatic ring, the redistribution of electrons around the ring allows for the activation of genotoxic side chains (Siim et al., 1997).

Type II nitroreductases are oxygen-sensitive, meaning that they reduce the nitro group in a one-electron step to a nitroanion radical, which in the presence of oxygen is back-oxidised back to its starting substrate, generating a superoxide anion in the process (Figure 1-3; Roldán et al., 2008). The generation of nitroso, hydroxylamine and/or amine products by these enzymes can therefore only occur under anaerobic conditions (Roldán et al., 2008). Type I nitroreductases, on the other hand, are oxygen-insensitive and reduce the nitro group via concerted two-electron transfers, bypassing the requirement for anoxic conditions, to ultimately form the hydroxylamine or amine products irrespective of whether oxygen is present (Figure 1-3; de Oliveira et al., 2010).

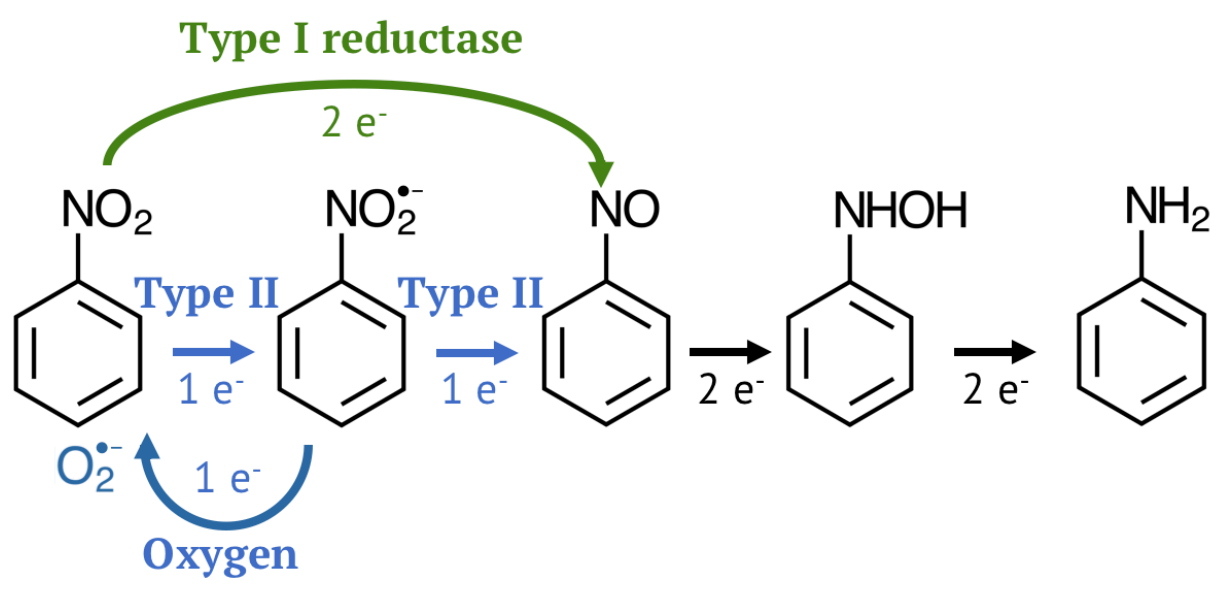

Figure 1-3 Schematic of type II (oxygen-sensitive; blue) versus type I (oxygenindependent; green) reduction of the nitroaromatic ring. Type II reduction proceeds via consecutive 1e-steps, and back-oxidation of the nitroanion radical occurs in the presence of oxygen (generating a superoxide anion). Type I reduction proceeds via consecutive $2 e$-steps and is oxygen-insensitive.

This reaction is undertaken via a ping pong bi-bi kinetic mechanism, where first $\mathrm{NAD}(\mathrm{P}) \mathrm{H}$ binds to and transfers 2 e- to the FMN cofactor and then leaves the active site, followed by binding of the nitroaromatic compound 
near the reduced FMN, and transfer of these $2 \mathrm{e}$ - to reduce the substrate (Koder and Miller, 1998; Pitsawong et al., 2014; Zenno et al., 1996).

As nitroaromatic compounds are relatively rare in nature (Ju and Parales, 2010), it is proposed that nitro-reduction is in fact a promiscuous activity; and that in most cases the primary biological role of nitroreductases involves the reduction of quinones to maintain a pool that contribute to antioxidant defence (Green et al., 2014; Wang and Maier, 2004). Consistent with this, it has been shown that $n f S A$ from $E$. coli is part of the soxRS regulon, which is induced in response to oxidative stress (Liochev et al., 1999; Paterson et al., 2002). In bioluminescent bacteria an additional or alternative role has been identified in which these enzymes can reduce free flavin to $\mathrm{FMNH}_{2}$ to power bioluminescence reactions (Zenno et al., 1994). Some nitroreductases have also been found to have the capacity to reduce iron (Takeda et al., 2010), azo dyes (Rafii and Cerniglia, 1995) or the environmental pollutant hexavalent chromium [Cr(VI)] (Ackerley et al., 2004; Robins et al., 2013; Zheng et al., 2015).

The ability of nitroreductases to reduce a wide range of structurally diverse non-native nitroaromatic substrates indicates their potential to reduce engineered prodrugs that possess more promising properties for GDEPT than CB1954. Furthermore, the natural promiscuity of these enzymes could enable rapid evolution for improved activation of CB1954 or other prodrugs through the alteration of a few residues, as has been seen with other promiscuous enzymes (Aharoni et al., 2005; Gould and Tawfik, 2005; Tracewell and Arnold, 2009).

\subsubsection{Challenges associated with evolution of nitroreductase enzymes}

Within different nitroreductase directed evolution projects conducted within the Ackerley group, we have observed the selection of nitroreductase variants that, although exhibiting substantial improvements in sensitising an $E$. coli host to a nitroaromatic compound, frequently do not demonstrate improved activity with the compound in vitro. Our lab postulates that this is 
often due to the other promiscuous roles that the over-expressed nitroreductase plays in the host, e.g. the reduction of intracellular quinones. Evolved variants may therefore not be directly improved with the nitroaromatic substrate, but instead have reduced activity with other competing cytosolic metabolites (Copp et al., 2017). Further directed evolution of these variants may subsequently allow for direct enzyme improvement with nitroaromatic substrate.

\subsection{Strategies to improve nitroreductase based GDEPT}

\subsubsection{Alternative bacterial nitroreductases for GDEPT}

A decade after the discovery of $E$. coli NfsB's ability to activate CB1954, a second nitroreductase, YwrO from the bacterial species Bacillus amyloliquefaciens, was also found to have activity with CB1954 (Anlezark et al., 2002). Purified YwrO protein showed a 1.5-fold improvement in $k_{c a t} / K_{M}$ over E. coli NfsB, however it reduced CB1954 exclusively at the 4-nitro position (Anlezark et al., 2002) which could limit its efficacy in generating a bystander effect. B. amyloliquefaciens YwrO and homologues of either itself or $E$. coli $\mathrm{NfsB}$ were investigated in a later study, in which the most promising enzyme, NfsB from Haemophilus influenzae, reduced CB1954 exclusively at the 4 -nitro position but exhibited a 9-fold increase $k_{c a t} / K_{M}$ over E. coli NfsB (Theys et al., 2006). H. influenzae NfsB only exhibited a modest improvement when co-incubated as a purified enzyme with V79 Chinese hamster ovary cells, CB1954 and NAD(P)H cofactor (Anlezark et al., 2002; Theys et al., 2006); however when the enzyme was integrated into a $C$. sporogenes vector it exhibited repeated strong antitumour responses in combination with CB1954 in a mouse xenograft model (Theys et al., 2006).

Other members of the NfsB family that exhibit improved $k_{c a t} / K_{M}$ activity over E. coli NfsB have also been discovered by other research groups (Heap et al., 2014; Prosser et al., 2013). NfsB from Neisseria meningitidis was measured as having an 880-fold improvement in $k_{\text {cat }} / K_{M}$ over $E$. coli NfsB, and it also exhibited improvement over $E$. coli $\mathrm{NfsB}$ when delivered via $C$. sporogenes 
vectors to xenograft mice, showing both increased tumour growth delay and overall survival following CB1954 administration (Heap et al., 2014). In this study, complete tumour cure was achieved in four out of sixteen mice administered $N$. meningtidis NfsB (Heap et al., 2014). Unlike previously investigated nitroreductases, $N$. meningtidis NfsB was measured as having a $K_{M}$ of $2.47 \mu \mathrm{M}$ (Heap et al., 2014), which could allow it to efficiently activate clinically relevant doses of CB1954 below the human maximum-tolerated dose (MTD).

The NfsA family of nitroreductases, exemplified by E. coli NfsA, are also able to reduce CB1954; they do so almost exclusively at the 2-nitro position (Barak et al., 2006a; Prosser et al., 2010a; Vass et al., 2009). E. coli NfsA has been measured as having (on average) a 12 -fold improvement in $k_{c a t} / K_{M}$ over E. coli NfsB (Williams et al., 2015). E. coli NfsA has also been shown to exhibit a 20 -fold greater cell killing over $E$. coli $\mathrm{NfsB}$ as a purified enzyme in co-culture with SKOV3 human ovarian carcinoma cells, CB1954 and NADPH (Vass et al., 2009). E. coli NfsA, following integration into a replicationdeficient adenovirus (AdSVO42) and transfection into 6.25\% of a SKOV3 population, generated a 7 -fold lower $\mathrm{IC}_{50}$ than $E$. coli $\mathrm{NfsB}$ in the same culture conditions (Vass et al., 2009). However, our group has seen reduced sensitivity of HCT-116 cell lines stably expressing E. coli NfsA to CB1954 relative to E. coli NfsB, which is most likely due to lower levels of NfsA enzyme expression in these cells (Prosser et al., 2010a, 2013). Other members of the NfsA family investigated by our group were consistently found to possess a much lower $K_{M}$ with CB1954 than members of the NfsB family, leading to a higher $k_{c a t} / K_{M}$ on average. The most active purified NfsA variant investigated by our group, NfsA from Vibrio fischeri, exhibited a 10fold higher $k_{c a t} / K_{M}$ than the most active NfsB variant, YfkO from Bacillus subtilis (Prosser et al., 2013). However, as with E. coli NfsA, these improvements in vitro did not consistently translate to increased sensitisation of stably expressed human cell lines to CB1954, due to highly variable enzyme expression (Prosser et al., 2010a, 2013; Williams et al., 2015). Another drawback of the NfsA family for GDEPT could be their 
preference for NADPH over NADH, as there is evidence suggesting a lower concentration of the former cofactor in both mammalian and bacterial cells (Klaidman et al., 1995; London and Knight, 1966; Ying, 2008).

Our group has also discovered multiple other minor Type I nitroreductases that possess some activity with CB1954; including Pseudomonas aeruginosa MsuE (Green et al., 2013), E. coli NemA (Prosser et al., 2010a; Robins et al., 2013), E. coli AzoR (Prosser et al., 2010a) and homologues of the latter two enzymes (Prosser et al., 2013). However, none of these enzymes have activity approaching the level of the NfsA or NfsB family members, so they do not generally appear to be attractive prospects for CB1954 GDEPT (Williams et al., 2015).

\subsubsection{Directed evolution of bacterial nitroreductases for GDEPT}

An alternative to the search for new nitroreductase enzymes is the engineering of existing ones to improve their capacity to activate CB1954. The Searle group at the University of Birmingham first looked to improve $E$. coli $\mathrm{NfsB}$ activity with CB1954 using a semi-rational approach, where they targeted nine residues in the substrate binding pocket of the solved crystal structure (Lovering et al., 2001). Saturation mutagenesis was utilised to individually alter the codons of these nine residues, allowing all 20 proteinogenic amino acids to be substituted in place of the wild-type residue at each site (Grove et al., 2003). Chromosomal integration of these variants into an $E$. coli $n f s B$-deletion strain was followed by negative selection using CB1954, where improved variants were selected for their inability to grow at concentrations of CB1954 that cells expressing wild-type NfsB could tolerate. The top selected variants were subsequently transfected into SKOV3 cells to evaluate their activity within human tumour cells (Grove et al., 2003). The lead variant, F124K, sensitised SKOV3 cells to a five-fold lower concentration of CB1954 than NfsB (Grove et al., 2003), and the purified protein was also shown to have a 2.4 -fold higher $k_{\text {cat }} / K_{M}$ than NfsB (Race et al., 2007). The Searle group also looked into the combination of 
beneficial individual mutations, and found two amino acid substitutions, F124K and N71K, that combined in a synergistic fashion (Race et al., 2007). Further evolution work conducted on $E$. coli NfsB led to the triple mutant T41Q/N71S/F124T, which showed a 45 -fold improvement in $k_{\text {cat }} / K_{M}$ (Jarrom et al., 2009); and the double mutant T41L/N71S which exhibited a 100-fold improvement in $k_{\text {cat }} / K_{M}$ over wild-type NfsB (Jaberipour et al., 2010; Jarrom et al., 2009). Transfection of T41L/N71S and another double mutant T41L/F70A into SKOV3 cells using an adenovirus vector sensitised these cells to 17-fold and 14-fold lower concentrations of CB1954 than E. coli $\mathrm{NfsB}$, respectively (Jaberipour et al., 2010). Another group later generated the triple mutant T41L/N71S/F124W and reported a 1.5 -fold higher $k_{\text {cat }} / K_{M}$ over the T41L/N71S double mutant; however no human cell transfections appear to have been conducted with this mutant thus far (Bai et al., 2015a). Our group's primary attempt to improve nitroreductase activity with CB1954 centred on the targeting of active site residues of another NfsB family enzyme, FRase I from $V$. fischeri (Swe et al., 2012). Three lead variants were generated from this research - F124W, A120V/F124G and A120V/K123N/F124W - the first exhibiting an 8-fold improvement in $k_{\text {cat }} / K_{M}$ over wild-type FRase I, and the latter two showing substantially lower $k_{\text {cat }}$ than FRase I, leading to only up to a 2 -fold improvement in $k_{\text {cat }} / K_{M}$ (Swe et al., 2012). Co-cultures of $E$. coli over-expressing these three variants with HCT-116 cells showed improved sensitivity to CB1954, with the double mutant generating a 2-fold lower $\mathrm{IC}_{50}$ than wild-type FRase I (Swe et al., 2012). Unfortunately the inability to stably express FRase I in HCT-116 cells limited our capacity to compare enzyme efficacy within the human tumour cell environment (Swe et al., 2012).

One final example of an enzyme engineered to have improved activity with CB1954 is YieF which, as a consequence of directed evolution to improve its ability to reduce the heavy metal pollutant Cr(VI) (Barak et al., 2006b), was found to have serendipitously gained activity with CB1954 as a substrate (Barak et al., 2006a). The mutant ChrR6 was found to sensitise cultured HeLa 
cervical cancer cells to an $\sim 10$-fold lower concentration of CB1954 than $E$. coli NfsA when both were delivered via an attenuated tumour-trophic Salmonella typhymurium vector (Barak et al., 2006a).

\subsubsection{Alternative nitroaromatic prodrugs}

Reduction of the nitro groups of prodrugs such as CB1954 can also be catalysed under hypoxic conditions by the human Type II nitroreductase enzyme, cytochrome $\mathrm{P} 450$ reductase. Reduction by this enzyme occurs in one-electron steps, the first step generating an unstable radical which is rapidly back-oxidised in the presence of oxygen to the starting substrate, meaning that full reduction to a DNA cross-linking metabolite can occur only in the absence of oxygen ( Figure 1-3; Stratford et al., 1981). SN23862, a nitrogen mustard analogue of CB1954 (chemical structure shown in Figure 1-4), was developed with the specific aim of recapitulating this mechanism to provide a hypoxia-activated prodrug (Siim et al., 1997). Reduction of either nitro group of SN23862 activates the DNA-crosslinking nitrogen mustard moiety (Brown and Wilson, 2004; Siim et al., 1997).<smiles>NC(=O)c1cc(N2CC2)c([N+](=O)[O-])cc1[N+](=O)[O-]</smiles>

CB1954<smiles>NC(=O)c1cc(N(CCCl)CCCl)c([N+](=O)[O-])cc1[N+](=O)[O-]</smiles>

SN23862<smiles>CO[Se]OCCN(CCBr)c1c(C(=O)NCCO)cc([N+](=O)[O-])cc1[N+](=O)[O-]</smiles>

PR-104A

Figure 1-4 Chemical structures of prodrugs CB1954, SN23862, and PR-104A.

An analogous hypoxia-activated nitrogen mustard, PR-104A (chemical structure shown in Figure 1-4), is administered to patients as a pre-prodrug, PR-104, which is rapidly hydrolysed in the patient's body to the prodrug 
form (McKeage et al., 2011). PR-104A progressed to Phase II clinical trials as a sole agent but was found to be activated aerobically by the human enzyme aldo-keto reductase 1C3 (AKR1C3; Guise et al., 2010), which acted as a Type I nitroreductase and limited the efficacy of PR-104 as an independent prodrug due to off-target activation in other tissues such as the bone marrow (McKeage et al., 2011). PR-104A was shown to be well-tolerated at doses up to $1,100 \mathrm{mg} / \mathrm{m}^{2}$, with an AUC of $20 \mathrm{mM} / \mathrm{h}$, however a Phase I/II trial in leukaemia patients showed tumour response to treatment only upon dosing at three to four-fold the MTD of PR-104A, generating grade 3/4 adverse effects in up to half of these patients (Konopleva et al., 2015).

PR-104A has a higher MTD and likely dose-potency than CB1954, making it an attractive candidate for repurposing as a nitroreductase GDEPT prodrug (Williams et al, 2015). Our research group have shown that a number of nitroreductases from the NfsA and NfsB families are capable of activating PR-104A independent of the oxygen status of the host cell (Prosser et al., 2013). Furthermore, HCT-116 human cell lines stably expressing NfsA from E. coli, NfrA from B. subtilis or NfsB from $V$. vulnificus were sensitised nearly 3000-fold to PR-104A relative to an untransfected control line (Prosser et al., 2013). Directed evolution of $E$. coli NfsA generated a variant that sensitised E. coli to up to 3.8-fold lower concentrations of PR-104A and showed a modest improvement (1.5-fold) in sensitising stably transfected human cell lines to the prodrug (Copp et al., 2017).

Building on this work, an analogue of PR-104A (SN34507; chemical structure shown in Figure 1-5) was rationally designed to limit off-target activation by AKR1C3, with the aim of increasing its human MTD for GDEPT applications. It was shown by our group to be activated by many different NfsA and NfsB family enzymes, including $E$. coli NfsA (Mowday et al., 2016b). In general, our lab group has found that orthologues of E. coli NfsB tend to exhibit substantially higher $k_{c a t}$ and $K_{M}$ with nitroaromatic prodrugs than orthologues of E. coli NfsA (Prosser et al., 2013). 
Mouse HCT-116 xenograft models comprising 30\% E. coli NfsA-expressing cells, following intraperitoneal administration of the pre-prodrug of SN34607, exhibited a doubling in tumour growth delay and a three-fold increase in median survival compared to PR-104 administration (Mowday et al., 2016b).<smiles>[2H]c1cc([N+](=O)[O-])cc(C(=O)NCCO)c1N(CCBr)CCO[R6](=O)OC</smiles>

Figure 1-5 Chemical structure of the nitrogen mustard prodrug SN34507.

A final possible application of GDEPT involves the targeting of tumour endothelial cells to induce collapse of the already instable vasculature around a solid tumour and reduce the flow of nutrients and oxygen that help sustain its growth (Hunt et al., 2012). The advantage of this target involves the much lower level of vector-mediated gene delivery required to generate the same therapeutic benefit as in targeting tumour epithelium (Hunt et al., 2012). In this regard, the investigation of nitroreductase/CB1954 and nitroreductase/metronidazole have yielded promising results in the collapse of tumour vascular network disruption following transient expression in human endothelial cell lines (Hunt et al., 2012). The latter prodrug, metronidazole, is a 5-nitroimidazole compound (chemical structure shown in Figure 1-6) that also cross-links DNA upon reduction of the nitro group; however downstream products of this reduction are not cell-permeable and do not cause a bystander effect (Bridgewater et al., 1997). This is an advantage in vascular-targeting GDEPT as metabolite escape in endothelial cells into the main circulation could lead to systemic exposure to cytotoxic metabolites (Hunt et al., 2012). 


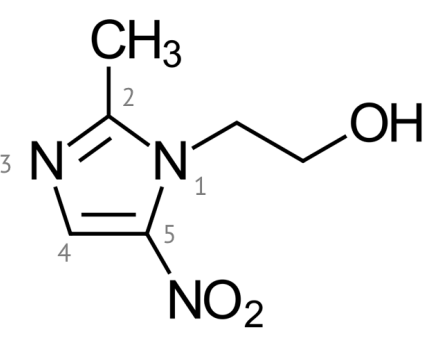

Figure 1-6 Chemical structure of the 5-nitroimidazole prodrug metronidazole.

\subsection{Non-invasive imaging strategies}

One current limitation of GDEPT clinical trials is the inability to noninvasively determine the location of the gene prior to prodrug administration, to minimise the risk of prodrug activation in off-target tissues (Belmar et al., 2007; Bhaumik, 2011; Penet et al., 2012; Yaghoubi et al., 2012). Currently, monitoring of the therapeutic vector in clinical trials still involves tissue biopsies or polymerase chain reaction (PCR)-based analysis of blood, urine and other body fluids (Heo et al., 2013; Morris et al., 2013; Roulstone et al., 2015). However, the invasive nature of taking biopsy samples limits the number of organs that can be sampled, and measurement of systemic viral or bacterial vector levels give no indication on vector localisation. Non-invasive whole-cell imaging would enable accurate, realtime determination of gene and vector localisation, and allow clinicians to assess the ideal time-point for prodrug administration.

\subsubsection{Optical imaging}

Fluorescent and bioluminescent imaging involves the emission and subsequent detection of a fluorescent or bioluminescent signal, either through oxidation of substrate (bioluminescence) or after excitation with a particular wavelength of light (fluorescence), and both are cost-effective options for gene imaging (Belmar et al., 2007; Brader et al., 2013; Saadatpour et al., 2016). Advantages of bioluminescence include its high signal-to-background ratio and absence of autofluorescence (Belmar et al., 
2007). It has been utilised for real-time imaging of gene expression in small animals such as rodents, however its inability to be imaged more than a few centimetres into tissue would limit its efficacy in imaging human patients (Cronin et al., 2012; Kuo et al., 2007). Fluorescent proteins, such as GFP, fare even worse, with shallower imaging depth than bioluminescence, and their signals can also be confounded by autofluorescence and photon scattering within tissues (Belmar et al., 2007; Leblond et al., 2010).

\subsubsection{Magnetic resonance imaging (MRI)}

MRI detects the time taken for unpaired nuclear spins to return to their alignment in a magnetic field after being disturbed by a radio-frequency pulse (Kristian Räty et al., 2007). The use of MRI can provide very high spatial resolution and is not restricted by depth of signal; it is also a much more widely used and accepted technique by clinicians (Belmar et al., 2007; Brader et al., 2013; Saadatpour et al., 2016). However it has lower sensitivity than nuclear imaging, and would require both cellular amplification and very high doses of MRI contrast agents to be administered to be clinically useful for GDEPT (Brader et al., 2013; Kristian Räty et al., 2007).

\subsubsection{Nuclear imaging}

Positron-emission tomography (PET) imaging detects the emission of positrons during the decay of radioisotopes such as ${ }^{14} \mathrm{O}$ and ${ }^{15} \mathrm{O},{ }^{13} \mathrm{~N}$, and ${ }^{18} \mathrm{~F}$ (Belmar et al., 2007). In a GDEPT context, radiolabelled probes would be activated by a reporter enzyme to a cell-entrapped form, and following elimination of the non-bound probe from a patient's body, the residual signal would then report on the location of the gene (Figure 1-7). To date this has shown the most promise for cancer gene therapy due to its high sensitivity. Limitations of this treatment involve both the requirement for a PET scanning machine, which comes with a significant operating cost, and the very short half-life of the most useful radioisotopes, which would require 
both the synthesis and administration of the probe to be conducted within a short time-frame of each other (Kristian Räty et al., 2007).

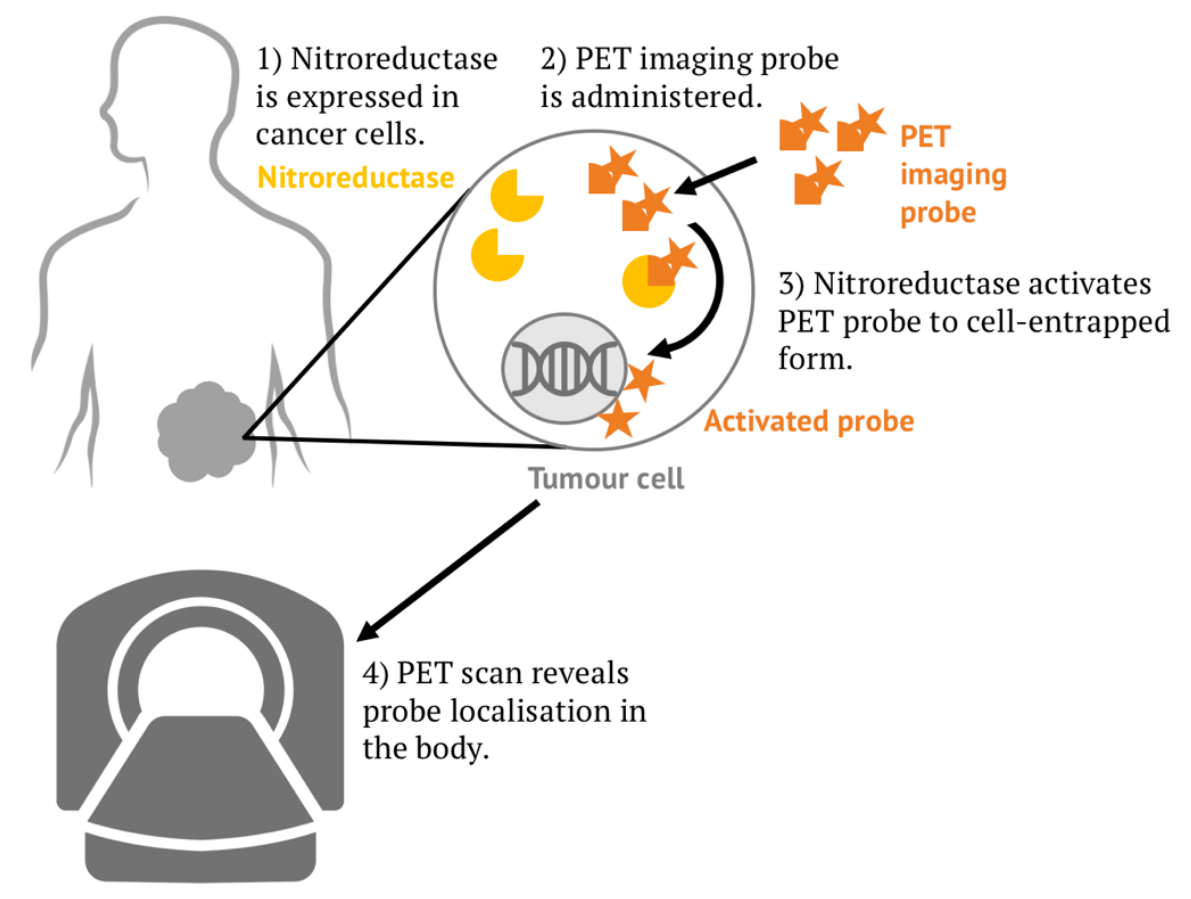

Figure 1-7 Schematic showing the nuclear imaging of a reporter gene/probe following activation by a nitroreductase.

\subsection{Imaging of GDEPT}

\subsubsection{Nuclear imaging of HSV1-TK therapies}

In addition to ganciclovir, HSVI-TK is capable of phosphorylating radiolabelled thymidine analogues such as ${ }^{124}{ }^{18}{ }^{18}$ F-FIAU [2'-fluoro-2'-deoxy1 - $\beta$-D-arabinofuranosyl-5-iodouracil] and ${ }^{18}$ F-FHBG [9-[4-fluoro-3(hydroxymethyl)butyl]guanine], both of which have the capacity to be detected as cell-entrapped forms in a PET scan. Researchers have shown increased entrapment of these probes in tumours expressing HSV1-TK or its engineered mutant derivative HSV1-sr39TK over other organs in mice (Tjuvajev et al., 2002; Yaghoubi et al., 2006) and in humans (Yaghoubi and Gambhir, 2006). 
A very recent study examining the use of ${ }^{18} \mathrm{~F}$-FHBG to monitor cytotoxic $\mathrm{T}$ lymphocytes (CTL) expressing HSV1-tk in seven recurrent glioma patients, provided a rudimentary proof-of-principle for the ability to track CTLs to tumour sites (Keu et al., 2017). However, accurate delivery of CTLs could not be assessed and researchers suggested that the use of HSV1-sr39TK, which has higher affinity for the probe, in future trials might allow for increased imaging sensitivity (Keu et al., 2017).

\subsubsection{Nuclear imaging of CD therapies}

There has been less success creating a CD-imageable reporter probe, with attempts at utilizing ${ }^{3} \mathrm{H}$-labelled 5-FC for both gene therapy and PET imaging showing no difference in uptake of 5-FC between a human glioblastoma cell line stably expressing bacterial CD, and control cells (Haberkorn et al., 1996). Some imaging success has been achieved by indirect measurement of CD localisation through intratumoural injection of a somatostatin receptor subtype 2 (SSTR2)-CD fusion protein in a replication-deficient adenovirus to mice xenograft tumours (Lears et al., 2015). Administration of the PET imaging probe ${ }^{64} \mathrm{C}-\mathrm{CB}-\mathrm{TE} 2 \mathrm{~A}-\mathrm{Y} 3-\mathrm{TATE}$ showed activation to a cell-entrapped form by SSTR2 in mice administered the adenovirus containing the fusion protein, and activation of ${ }^{3} \mathrm{H}-5-\mathrm{FC}$ by the CD enzyme could also be detected, showing that both parts of the fusion protein were still active (Lears et al., 2015).

\subsubsection{Optical imaging of nitroreductases}

Most of the work conducted on the development of nitroreductase-activated probes for gene therapy imaging has focused on fluorescent probes in which the fluorescence is quenched by the presence of the nitro group in the structure, and restoration of fluorescence occurs following nitroreductase reduction of the compound. One class of probes investigated is the nearinfrared fluorescent probes (Guo et al., 2013; Li et al., 2015a, 2015b), 
including CytoCy5 (Bhaumik et al., 2012; McCormack et al., 2013; Stanton et al., 2015), which have shown detectable nitroreductase-specific fluorescence in mice and zebrafish. Development of bioluminescent probes (Vorobyeva et al., 2015) and chemiluminescent probes (Cao et al., 2016) have also shown promise for imaging in animals. However as previously discussed in Section 1.5.1, clinical application of these probes in humans would be limited by the poor tissue penetrating properties of visible wavelengths of light.

\subsubsection{Nuclear imaging of nitroreductases}

Previous members in the Ackerley group have explored the possibility of PET imaging of nitroreductases, through the repurposing of probes originally developed to image hypoxia. Much like the hypoxia-activated prodrugs described in Section 1.4.3, hypoxia-activated 2-nitroimidazole imaging probes can be activated by endogenous one-electron reductases to intermediates that are oxidized back to the starting substrate by molecular oxygen. Only in hypoxic conditions are these probes further converted to alkylating products that react with intracellular thiols and become cellentrapped (Krohn et al., 2008; Lapi et al., 2009). Three of these probes, EF5 [2-(2-nitro-(1)H-imidazol-1-yl)-N-(2,2,3,3,3-pentafluoropropyl)-acetamide] (Koch et al., 2001, 2010), F-MISO (fluoro-misonidazole) (Rajendran et al., 2006; Thorwarth et al., 2005), and acr(van Loon et al., 2010), can be radiolabelled with ${ }^{18} \mathrm{~F}$ (Krohn et al., 2008; Lapi et al., 2009), and have all been evaluated clinically for detection of hypoxia (chemical structures of all three radiolabelled probes shown in Figure 1-8).

Research conducted by our group showed that multiple NfsA (but not NfsB) enzymes from different bacterial species could reduce EF5, F-MISO, and HX4 (Williams, 2013). Based on this, our collaborators at the Auckland Cancer Society Research Centre (ACSRC) determined that a HCT-116 cell line stably expressing E. coli NfsA exhibited significantly increased EF5 entrapment over HCT-116 cells alone or cells expressing E. coli NfsB (Figure 1-9; Williams, 2013). 
<smiles>O=[N+]([O-])c1nccn1CC(O)CF</smiles>

$\left[{ }^{18}\right.$ F]F-MISO<smiles>O=C(Cn1ccnc1[N+](=O)[O-])NCC(F)(F)F</smiles>

$\left[{ }^{18} \mathrm{~F}\right] \mathrm{EF} 5$<smiles>O=[N+]([O-])c1nccn1Cc1cn(C(CO)CF)nn1</smiles>

$\left[{ }^{18} \mathrm{~F}\right] \mathrm{HX} 4$

Figure 1-8 Chemical structures of ${ }^{18} \mathrm{~F}$ 2-nitroimidazole hypoxia imaging agents $\mathrm{F}$ MISO, EF5 and HX4.
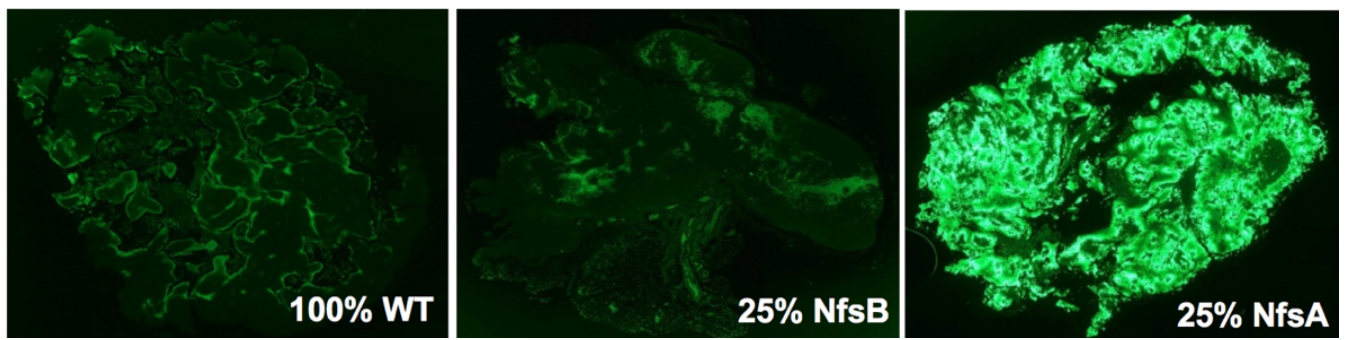

Figure 1-9 In vivo binding of EF5 in HCT-116:nitroreductase tumour xenografts. HCT-116 tumours were established in NIH III nude mice via subcutaneous injection of $10 \times 10^{6}$ cells in $100 \mu \mathrm{L}$ serum free media. Cells added comprised A) 100\% wild type HCT-116 cells B) $25 \%$ HCT-116:NfsA_Ec cells C) $25 \%$ HCT116:NfsB_Ec cells. Mice were treated with $60 \mathrm{mg} / \mathrm{kg}$ EF5 by intraperitoneal injection and left for three hours. Tumours were then excised and fixed in 10\% formalin for $48 \mathrm{~h}$, then paraffin embedded, sectioned and mounted on glass slides for immunohistochemical analysis with anti-EF5 antibody Alexa-488 ELK3.51 $(100 \mu \mathrm{g} / \mathrm{ml})$. Sections were scanned on a Nikon 2000E Inverted microscope. Reproduced with permission from Williams, 2013.

Subsequently, our collaborators at the University of Maastricht showed in ${ }^{18} \mathrm{~F}-\mathrm{HX} 4$ micro-PET experiments that mouse tumour xenografts comprising 100\% NfsA-expressing HCT-116 cells possessed significantly higher tumourto-blood ratios of ${ }^{18} \mathrm{~F}$ than tumours comprising $100 \% \mathrm{NfsB}$-labelled or untransfected HCT-116 cells ( $\mathrm{p}<0.005$; unpublished data). Nevertheless, the lowest recorded ratios from individual NfsA-labelled tumours were lower than the highest recorded ratios for the untransfected control tumours, suggesting that the background signal due to hypoxia might prove confounding (i.e., that it might not be possible to conclusively determine the NfsA-expression status of a tumour based on isolated PET scans). As an alternative strategy, our ACSRC collaborators have developed bespoke 5- 
nitroimidazole PET-capable analogues of EF5 that are non-responsive to endogenous human nitroreductases even under hypoxic conditions (Anderson et al., 2014). Identification and optimization of nitroreductases to partner with these molecules was a primary focus of the research described in this thesis.

\subsection{Aims of this study}

The research described in this thesis initially sought to utilise specialised $E$. coli screening strains and production of $\beta$-galactosidase or green fluorescent protein (GFP) to select for variants from nitroreductase candidate libraries with improved abilities to activate novel 5-nitroimidazole PET imaging probes. However, over the course of this work various issues with these systems emerged, including the high selection of false positives during screening.

I therefore tested and implemented an alternative strategy for selection of superior nitroreductase variants, based on our discovery of two positive selection substrates whose cytotoxic effects on $E$. coli could be mitigated by the presence of an over-expressed nitroreductase, and that I showed could be used to eliminate inactive or poorly-active nitroreductase variants from a large pool of mutants. I then used this strategy for the selection of active nitroreductases from nitroreductase variant libraries and from randomly cloned metagenomic DNA fragments.

The final aims were to:

- Evaluate the ability of wild-type nitroreductase enzymes to activate novel 5-nitroimidazole PET imaging probes, and screen site-targeted libraries of the most promising enzymes for improved variants.

- Utilise an error-prone directed evolution approach to engineer promising nitroreductase enzymes for further specificity with 5nitroimidazole PET imaging probes. 
- Validate the ability of nitroreductase-selective compounds niclosamide and chloramphenicol to select for nitroreductases active with both 5-nitroimidazole PET imaging probes and other nitroaromatic compounds of interest.

- Utilise nitroreductase-selective compounds for the discovery of nitroreductases from metagenomic DNA. 


\section{Chapter 2. Methods}

\subsection{Chemical reagents and enzymes}

The compounds S33, SN36506 and SN37519 were characterised by ${ }^{1} \mathrm{H}$ NMR and mass spectrometry and provided for this study by Dr Jeff Smaill (University of Auckland, New Zealand). All other nitroaromatic compounds including metronidazole, 4(5)-nitroimidazole, 2-methyl-4(5)nitroimidazole, niclosamide, chloramphenicol, azomycin, nitrofurazone, nitrofurantoin, and CB 1954 were purchased from Sigma-Aldrich (St. Louis, $M O, U S A)$. Unless otherwise stated, stock solutions of all drug and imaging agent compounds were made up in dimethyl sulfoxide (DMSO) and stored at $-80^{\circ} \mathrm{C}$.

Restriction enzymes were purchased from New England Biolabs (NEB; Ipswich, MA, USA) and Thermo Fisher Scientific (Waltham, MA, USA). BioMix $^{\mathrm{TM}}$ Red Polymerase Mastermix was purchased from Bioline (London, $U K)$. Phusion ${ }^{\mathrm{TM}}$ high-fidelity DNA polymerase was purchased from New England Biolabs (NEB; Ipswich, MA, USA). Agilent Technologies GeneMorph ${ }^{\circledR}$ II Random Mutagenesis Kit was purchased from Integrated Sciences (Sydney, NSW, AU). Bugbuster ${ }^{\circledR}$ HT Protein Extraction Reagent was purchased from Merck Millipore (Billerica, Massachusetts, USA). Unless otherwise stated, all other chemicals and reagents were purchased from Sigma-Aldrich (St. Louis, MO, USA), Thermo Fisher Scientific (Waltham, MA, USA), or Total Lab Systems (Auckland, NZ).

\subsection{Oligonucleotide primers}

Oligonucleotide primers used in this study were supplied as lyophilised powders by Integrated DNA Technologies (IDT; Coralville, IA, USA). Stock solutions of $100 \mu \mathrm{M}$ were made up in Tris-EDTA (TE; $10 \mathrm{mM}$ Tris- $\mathrm{HCl} \mathrm{pH}$ 8.0, 0.1 mM EDTA) and $10 \mu \mathrm{M}$ working solutions were diluted in $\mathrm{diH}_{2} \mathrm{O}$. Working and stock solutions were stored at $-20^{\circ} \mathrm{C}$. The sequences of all primers used in this study are listed in Table 2-1. 
Table 2-1 Primers used in this study

\begin{tabular}{|c|c|}
\hline Primer name & $\begin{array}{l}\text { Sequence }\left(5^{\prime} \rightarrow 3^{\prime}\right) \text { (underlined text indicate } \\
\text { restriction sites used for cloning) }\end{array}$ \\
\hline pmmB_Fw & GGCTCGTATAATGTGTGG \\
\hline pmmB_Rv & GACCGCTTCTGCGTTCTGAT \\
\hline pUCX_Fw & GACATCATAACGGTTCTG \\
\hline pUCX_Rv & GTTTCACTTCTGAGTTCG \\
\hline YcnD_Bs_Fw & CCCCCATATGAATGAAGTGATTAAATC \\
\hline YcnD_Bs_Rv & CCCCGTCGACTTATTTTTCAACTTTAAATC \\
\hline YfkO_Bs_Fw & GGGGCATATGGCAGATCTAAAGACACA \\
\hline YfkO_Bs_Rv & CCCCGTCGACTTAAACCCACTTCACAACAT \\
\hline NfsA_Ec_Fw & GGCATATGACGCCAACCATTGAAC \\
\hline NfsA_Ec_Rv & GGGTCGACTTAGCGCGTCGCCCAACCCTG \\
\hline T7_promoter & TAATACGACTCACTATAGGG \\
\hline T7_terminator & GCTAGTTATTGCTCAGCGG \\
\hline ACYCDuetUP1 & GGATCTCGACGCTCTCCCT \\
\hline DuetDOWN1 & GATTATGCGGCCGTGTACAA \\
\hline GFP_UPSphI & ATATAGCATGCGTAAAGGAGAAGAACTTTTCA \\
\hline GFP_DOWNHindIII & CTCTCAAGCTTATTTGTATAGTTCATCCATGC \\
\hline GFP_DOWN_LVA & $\begin{array}{l}\text { CTCTCAAGCTTATTAAGCTGCTAAAGCGTAGTTTT } \\
\text { CGTCGTTTGCTGCTTTGTATAGTTC }\end{array}$ \\
\hline GFP_DOWN_LAA & $\begin{array}{l}\text { CTCTCAAGCTTATTAAACTGCTGCAGCGTAGTTTT } \\
\text { CGTCGTTTGCTGCTTTGTATAGTTC }\end{array}$ \\
\hline GFP_DOWN_ASV & $\begin{array}{l}\text { CTCTCAAGCTTATTAAGCTACTAAAGCGTAGTTTT } \\
\text { CGTCGTTTGCTGCTTTGTATAGTTC }\end{array}$ \\
\hline GFP_DOWN_AAV & $\begin{array}{l}\text { CTCTCAAGCTTATTAAACTGATGCAGCGTAGTTTT } \\
\text { CGTCGTTTGCTGCTTTGTATAGTTC }\end{array}$ \\
\hline NfsA_Es_Fw & CCCCCATATGACGCCAACGATTGAGCTGC \\
\hline NfsA_Es_Rv & GGGGCTCGAGTTAGCGTGTCGCCCAGCCCTG \\
\hline NemA_St_Fw & CCCATTAATATGTCATCAGCAAAACTG \\
\hline NemA_St_Rv & CCCGGTCGACTTACAGAGTAGGGTAGTC \\
\hline YwrO_Bs_Fw & CCСATTAATATGAAAATATTGGTTTTGGCA \\
\hline YwrO_Bs_Rv & CCCGTCGACTTAAACAAAAGGCTGCTG \\
\hline YwrO_Li_Fw & CCCCCATATGAAAACATTAGTTATTAT \\
\hline YwrO_Li_Rv & CCCGTCGACCTAATTTAACGTTTTAATA \\
\hline NfsB_Nme_Fw & CCCCATATGACAGTATTATCT \\
\hline NfsB_Nme_Rv & CCCGTCGACTTAAGCCCATATTA \\
\hline YfkO_Bli_Fw & GGGGCATATGACAGAGCAATCCAAG \\
\hline YfkO_Bli_Rv & CCCCGTCGACTTATTCGACCCATTTC \\
\hline YcnD_Bli_Fw & CCCCATATGAATGAAGTATTGAA \\
\hline YcnD_Bli_Rv & CCCGTCGACTTATTCGAGTTTAAATCC \\
\hline NfrA_Bli_Fw & CCCCATATGAATAAAACGATTG \\
\hline NfrA_Bli_Rv & CCCGTCGACTTACCTTTTGTTCAAAC \\
\hline YwrO_Bam_Fw & CCCCCATATGAAAGTATTGGTA \\
\hline YwrO_Bam_Rv & CCCCGTCGACTTAAAATGATTT \\
\hline YcnD_Bs_19G_Fw & TCGATTCGCAGCTATACAGGTGAACCT \\
\hline
\end{tabular}




\begin{tabular}{ll} 
Primer name & $\begin{array}{l}\text { Sequence }\left(\mathbf{5}^{\prime} \rightarrow \mathbf{3}^{\prime}\right) \text { (underlined text indicate } \\
\text { restriction sites used for cloning) }\end{array}$ \\
\hline YcnD_Bs_19Rv & TGTATAGCTGCGAATCGA \\
\hline YcnD_Bs_163V_Fw & CCTGTATCCGGCCTTGTCGTCGGCCAT \\
YcnD_Bs_163Rv & AGCAAGGCCGGATACAGG \\
\hline YcnD_Bs_227V_Fw & GGTCACAGGGCATCGCATCCGTGTATGAG \\
YcnD_Bs_227L_Fw & GGTCACAGGGCATCGCATCCTTGTATGAG \\
\hline YcnD_Bs_227Rv & GGATGCGATGCCCTGTGACC \\
YcnD_Bs_239VRv & CCCCGTCGACTTATTTTTCAACTTTAA
\end{tabular}

\subsection{Software used for creation of figures, sequence alignments and enzyme} active site modelling

\subsubsection{Creation of figures}

Plasmid maps were generated using the open-source visualisation software AngularPlasmid (http://angularplasmid.vixis.com/; Vixis, LLC). Graphs were generated using either GraphPad Prism 7.0 (GraphPad Software Inc.; La Jolla, CA, USA), or the open source software RStudio (RStudio, Inc., Boston, MA, USA).

\subsubsection{Sequence alignments}

All nucleotide or amino acid sequence alignments were generated using Geneious 8.0 (Biomatters Ltd; Auckland, NZ).

\subsubsection{Enzyme active site modelling}

Enzyme crystal structures were downloaded from RSCB Protein Database (PDB; http://www.rcsb.org/) and visualised using USCF Chimera (Resource for Biocomputing, Visualization, and Informatics (RBVI); University of California, San Francisco, USA). The YcnD_Bs dimer was generated from a monomer crystal structure (PDB structure ID 1ZCH; Morokutti et al., 2005) by visualizing all symmetry mates in PyMOL (Schrödinger, LLC; New York City, New York, USA) and selection of the match that most resembled the structure of a nitroreductase. Amino acid residue substitutions were created 
using the Rotamers function in Chimera and the Dunbrack rotamer library

(Dunbrack, 2002) and the amino acid rotation that had the highest

probability of occurrence was selected.

\subsection{Strains and plasmids used in this study}

\subsubsection{Bacterial strains}

All E. coli expression and screening strains used in this study are described in

Table 2-2, and all bacterial strains used as sources of genomic DNA in this study are listed in Table 2-3.

Table 2-2 Escherichia coli strains used in this study

\begin{tabular}{|c|c|c|}
\hline Strain & Description & Source \\
\hline SOS-R2 & $\begin{array}{l}\text { ADA-510 } \Delta n f s A \Delta n f s B \Delta a z o R \Delta n e m A \\
\Delta \text { tolC }\end{array}$ & $\begin{array}{l}\text { (Prosser et al., } \\
2013 \text { ) }\end{array}$ \\
\hline $7 \mathrm{NT}$ & $\begin{array}{l}\text { W3110 } \Delta n f s A \Delta n f s B \Delta y i e F \Delta y c a K \Delta m d a B \\
\Delta a z o R \Delta n e m A \Delta t o l C\end{array}$ & (Copp et al., 2014) \\
\hline SOS-R4 & 7NT bearing pANODuet-1:GFPmut3b & (Copp et al., 2014) \\
\hline DH5 $\alpha$ & 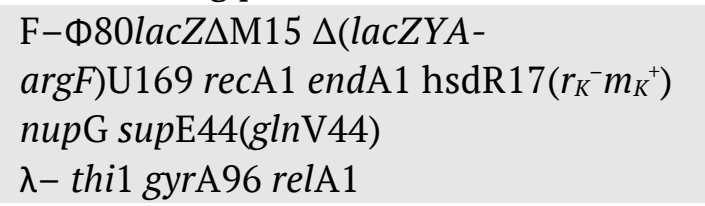 & Invitrogen \\
\hline $\begin{array}{l}\text { BL21 } \\
\text { (DE3) }\end{array}$ & $\begin{array}{l}\mathrm{F}^{-} \text {omp } \mathrm{T} \text { gal dcm lon hsd } \mathrm{S}_{\mathrm{B}}\left(\mathrm{r}_{\mathrm{B}}^{-} \mathrm{m}_{\mathrm{B}}^{-}\right) \\
\lambda(\mathrm{DE} 3)\end{array}$ & Novagen \\
\hline $7 \mathrm{TL}$ & $7 \mathrm{NT} \lambda(\mathrm{DE} 3)$ & (Little, 2015) \\
\hline EC100 & $\begin{array}{l}\mathrm{F}^{-} \text {mcrA } \Delta(\text { mrr- } h s d \mathrm{RMS}-\text { mcrBC }) \\
\text { Ф80dlacZ } \Delta \mathrm{M} 15 \Delta \text { lacX74 recA1 endA1 } \\
\text { araD139 } \Delta(\text { ara, leu }) 7697 \text { galU gal } \mathrm{K} \\
\lambda^{-} \text {rpsL }\left(\mathrm{Str}^{\mathrm{R}}\right) \text { nup } \mathrm{G}\end{array}$ & Epicenter \\
\hline
\end{tabular}

Table 2-3 Bacterial strains used as sources of genomic DNA in this study

\begin{tabular}{ll} 
Strain & Source \\
\hline Escherichia coli W3110 & Lab stock \\
Bacillus subtilis subsp. subtilis str. 168 & Brady lab \\
Citrobacter koseri ATCC 27156 & Lab stock \\
Pseudomonas putida KT2440 & Lab stock \\
Burkholderia thailandensis E264 & Brady lab
\end{tabular}




\subsubsection{Plasmids}

All plasmids used for transformation of $E$. coli cells are listed in Table 2-4.

Also shown are plasmid maps of the vectors used for expression of bacterial nitroreductase (NTR) enzymes, metagenomic fragments, and GFP in E. coli.

Table 2-4 Plasmids used for cloning in this study

\begin{tabular}{|c|c|c|c|}
\hline Plasmid & Resistance & Description & Source \\
\hline pUCX & $A m p^{R}$ & $\begin{array}{l}\text { Nitroreductase expression } \\
\text { vector. pUC ori, lacI gene, } \\
\text { tac promoter, lac operator, } \\
\text { pET28a+ RBS, rrnB } \\
\text { terminator. }\end{array}$ & $\begin{array}{l}\text { (Prosser et } \\
\text { al., 2010a) }\end{array}$ \\
\hline pUCX:KG & $\mathrm{Amp}^{\mathrm{R}}, \mathrm{Kan}^{\mathrm{R}}$ & $\begin{array}{l}\text { pUCX with nitroreductase } \\
\text { gene insert which } \\
\text { reconstitutes truncated } \\
\text { kan }^{R} \text { gene downstream of } \\
\text { MCS. }\end{array}$ & $\begin{array}{l}\text { (Prosser et } \\
\text { al., 2015) }\end{array}$ \\
\hline pET28a+ & $\operatorname{Kan}^{\mathrm{R}}$ & $\begin{array}{l}\text { Expression vector for } \mathrm{His}_{6}{ }^{-} \\
\text {tagged enzyme } \\
\text { purification. pBR322 ori, } \\
\text { T7 promoter, lac operator. }\end{array}$ & Novagen \\
\hline pCDFDuet-1 & $\operatorname{Spec}^{\mathrm{R}}$ & $\begin{array}{l}\text { Expression vector for co- } \\
\text { expression of two target } \\
\text { ORFs, each preceded by } T 7 \\
\text { promoter, lac operator and } \\
\text { rbs. pCDF ori. }\end{array}$ & Novagen \\
\hline $\begin{array}{l}\text { pANODuet- } \\
1: G F P\end{array}$ & $\mathrm{Spec}^{\mathrm{R}}, \mathrm{Cm}^{\mathrm{R}}$ & $\begin{array}{l}\text { GFPmut3b under control of } \\
\text { sulA SOS promoter, and } \\
\mathrm{cm}^{R} \text { cloned into MCS1 of } \\
\text { pCDFDuet- } 1 .\end{array}$ & $\begin{array}{l}\text { (Copp et al., } \\
\text { 2014) }\end{array}$ \\
\hline
\end{tabular}




\subsubsection{Plasmid map of expression vector pUCX:AzoR_EC (high expression)}

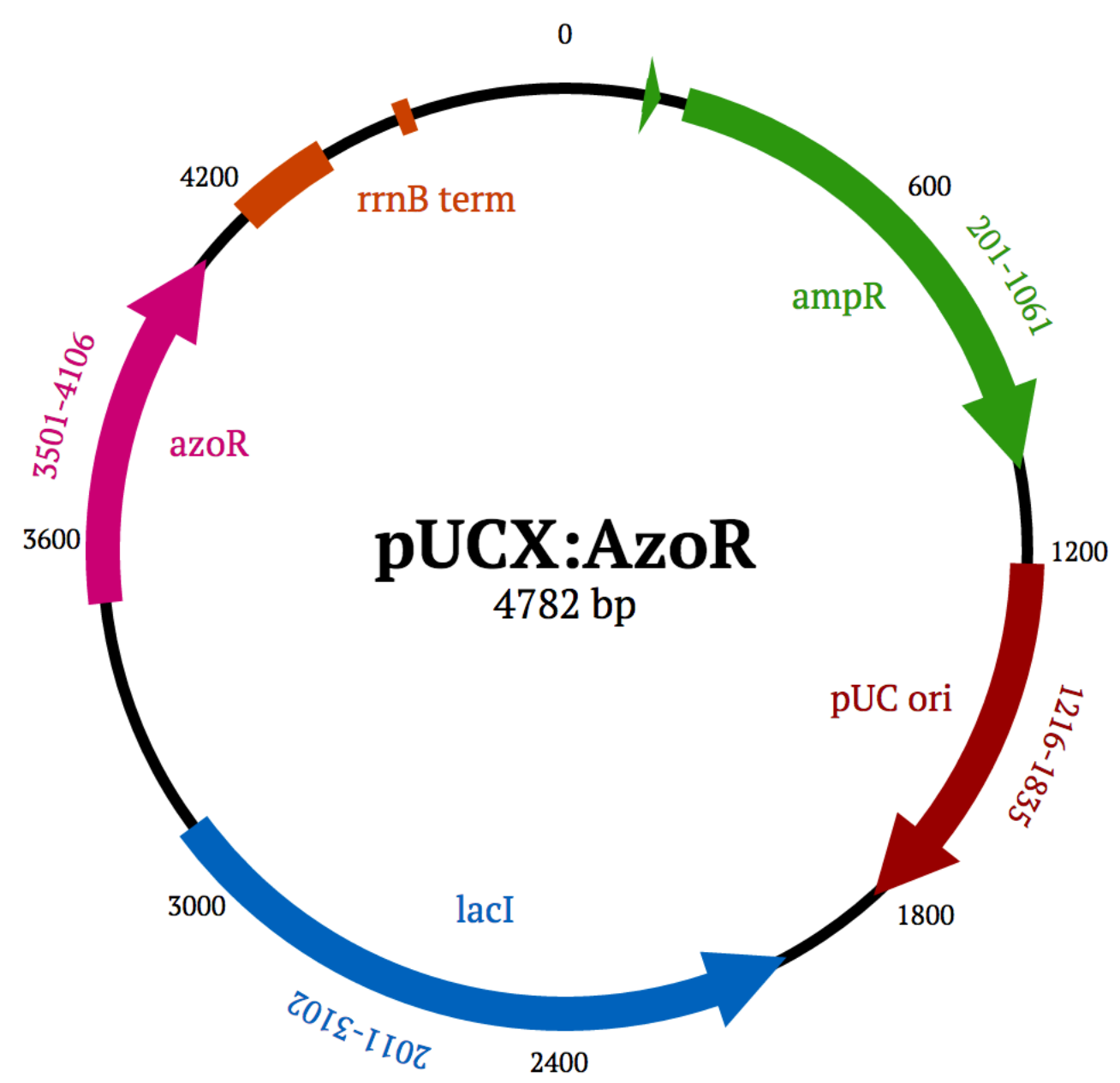

Figure 2-1 Plasmid map of nitroreductase expression vector pUCX:AzoR_Ec (high expression). Numbers denote the base pair location of genes of interest. Key features of the plasmid include the ampicillin resistance cassette (green), pUC ori (red), lacI repressor (blue), AzoR_Ec nitroreductase in the MCS (pink), and the rrnB terminators (orange). Differences between low-expressing and highexpressing $p U C X$ vectors are explained in Section 3.3.1.1. 


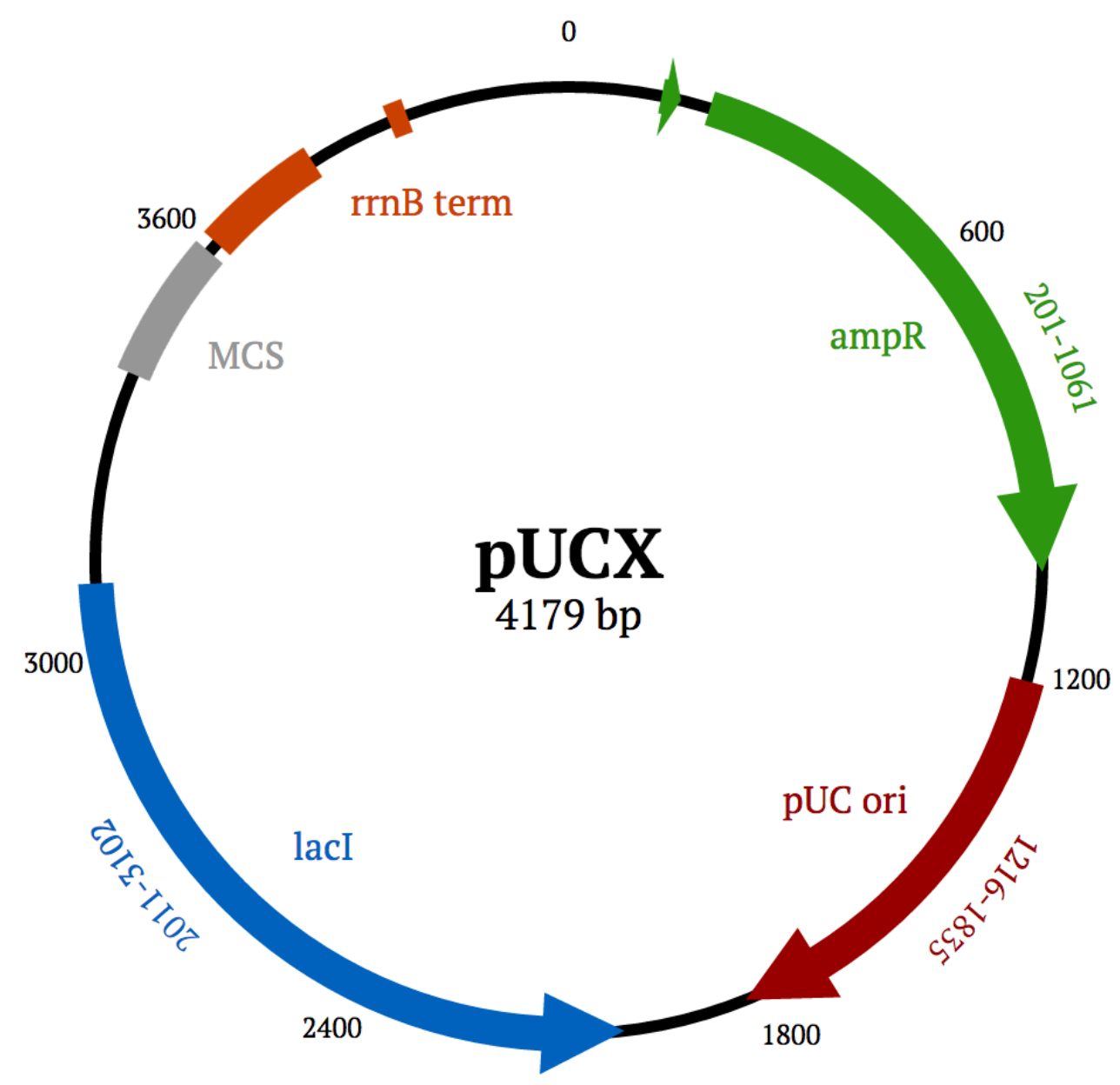

Figure 2-2 Plasmid map of nitroreductase expression vector pUCX (high expression). Numbers denote the base pair location of genes of interest. Key features of the plasmid include the ampicillin resistance cassette (green), pUC ori (red), lacI repressor (blue), multiple cloning site (includes promoter, operator and ribosome binding sites, shown in greater detail in Figure 2-3), and the rrnB terminators (orange). Differences between low-expressing and high-expressing pUCX vectors are explained in Section 3.3.1.1. 


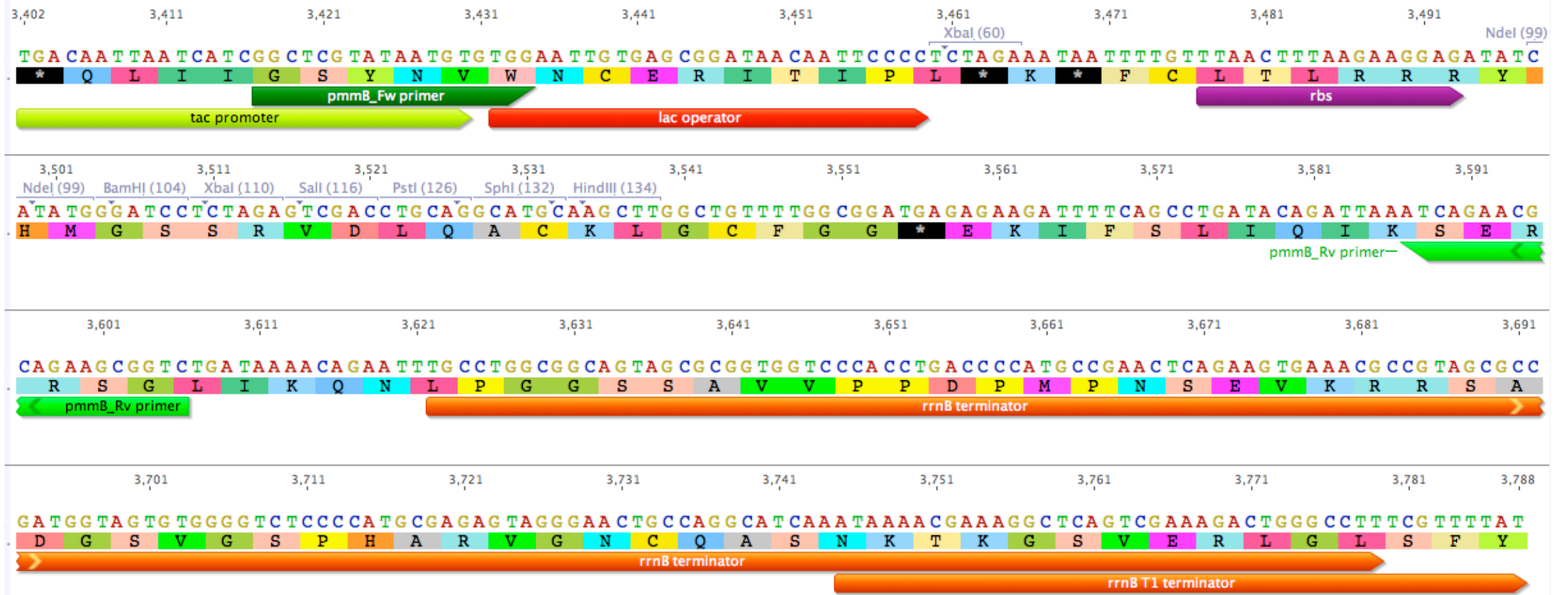

Figure 2-3 Multiple-cloning site of nitroreductase expression vector pUCX (low expression). Numbers denote the base pair location of sequences of interest. Key features of the multiple-cloning site (MCS) include the tac promoter (light green), pmmB_Fw primer binding site (dark green), lac operator (red), ribosome binding site (purple), pmmB_Rv primer binding site (green), rrnB and rrnB T1 terminators (orange), and restriction enzyme sites. NdeI, BamHI, SalI, PstI, SphI, and HindIII restriction enzyme cleavage sites are unique to the MCS. 


\subsubsection{Multiple-cloning site of E. coli expression vector pUCX (high expression)}

TGACAATTATCATCGGCTCGTATAATGTTGGAATG TGAGCGGATAACAATCCCCTCTAGAAATATTTGTTTAATTTAAAAGGAGAT

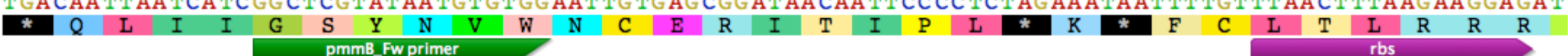
tac promoter lac operator

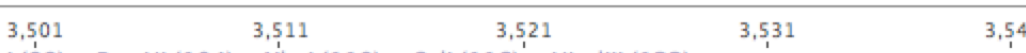
$3,5,41$ $3,5,61$ 3,581

ATCATATGGEATCCTCTAGAGTCGACAAGCTTGGCTGTTTTGCGGATGAGAGAAGATTTCAGCCTGATACAGATTAAATCAGAACGCAGAAG \begin{tabular}{lllllllllllllllllllllllllllllllllllllllll}
$\mathrm{Y}$ & $\mathrm{H}$ & $\mathrm{M}$ & $\mathrm{G}$ & $\mathrm{S}$ & $\mathrm{S}$ & $\mathrm{R}$ & $\mathrm{V}$ & $\mathrm{D}$ & $\mathrm{K}$ & $\mathrm{L}$ & $\mathrm{G}$ & $\mathrm{C}$ & $\mathrm{F}$ & $\mathrm{G}$ & $\mathrm{G}$ & $\boldsymbol{*}$ & $\mathrm{E}$ & $\mathrm{K}$ & $\mathrm{I}$ & $\mathrm{F}$ & $\mathrm{S}$ & $\mathrm{L}$ & $\mathrm{I}$ & $\mathrm{Q}$ & $\mathrm{I}$ & $\mathrm{K}$ & $\mathrm{S}$ & $\mathrm{E}$ & $\mathrm{R}$ & $\mathrm{R}$ & $\mathrm{S}$ \\
\hline
\end{tabular}

\begin{tabular}{llllllll}
\hline $3,5,91$ & 3,601 & 3,611 & 3,6121 & 3,631 & 3,641 & 3,651 & 3,61
\end{tabular}

CGGTCTGATAAAACA GAATTGCCTGGCGGCAGTAGCGCGGTGGTCCCACCTGACCCCATGCCGAACTCAGAAGTGAACGCCGTAGCGCCGAT \begin{tabular}{llllllllllllll|llllllllllllllllllll} 
G & L & I & K & Q & N & L & P & G & G & S & S & A & V & V & P & P & D & P & M & P & N & S & E & V & K & R & R & S & A & D &
\end{tabular} - pmmB_Rv primer

3,691

3,701

3,711

3,721

3.731

3.741

3,751

3.761

G GTAGTTGG G T CTCCCCATGCGAGAGTAG GGACTGCCAGGCATCAAATAAAACGAAAGGCTCAGTCGAAAGACTGGGCCTTTCGTTTAT $\begin{array}{llllllllllllllllllllllllllllllllllllll}\text { G } & \text { S } & \text { V } & \text { G } & \text { S } & \text { P } & \text { H } & \text { A } & \text { R } & \text { V } & \text { G } & \text { N } & \text { C } & \text { Q } & \text { A } & \text { S } & \text { N } & \text { K } & \text { T } & \text { K } & \text { G } & \text { S } & \text { V } & \text { E } & \text { R } & \text { L } & \text { G } & \text { L } & \text { S } & \text { F } & \text { Y } & \end{array}$

Figure 2-4 Multiple-cloning site of nitroreductase expression vector pUCX (high expression). Numbers denote the base pair location of sequences of interest. Key features of the MCS include the tac promoter (light green), pmmB_Fw primer binding site (dark green), lac operator (red), ribosome binding site (purple), pmmB_Rv primer binding site (green), rrnB and rrnB T1 terminators (orange), and restriction enzyme sites. NdeI, BamHI, SalI, and HindIII restriction enzyme cleavage sites are unique to the MCS. 


\subsubsection{Plasmid map and multiple-cloning site of E. coli SOS plasmid pANODuet-}

\section{1:GFP}

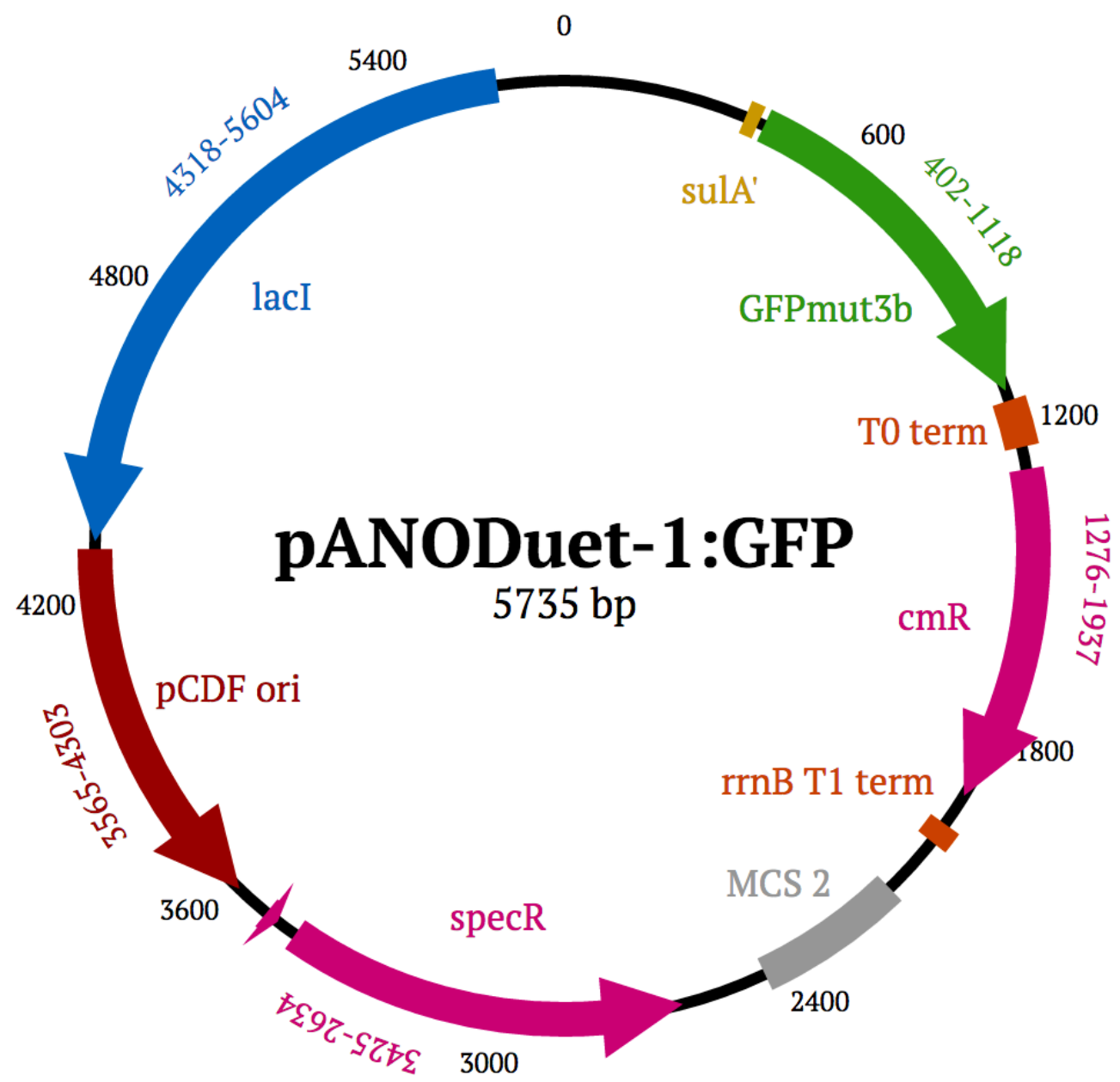

Figure 2-5 Plasmid map of SOS-inducible GFP plasmid pANODuet-1:GFP. Numbers denote the base pair location of genes of interest. Key features of the plasmid include the sulA' SOS promoter (yellow), GFPmut3b gene (green), lambda TO and $\mathrm{rrnB} T 1$ terminators (orange), chloramphenicol ( $\mathrm{cm}$ ) and spectinomycin (spec) resistance cassettes (pink), MCS2 of pCDFDuet-1 (grey), pCDF ori (red), and lacI repressor (blue). 


\subsubsection{Plasmid map and multiple-cloning site of E. coli expression vector} pET28a+

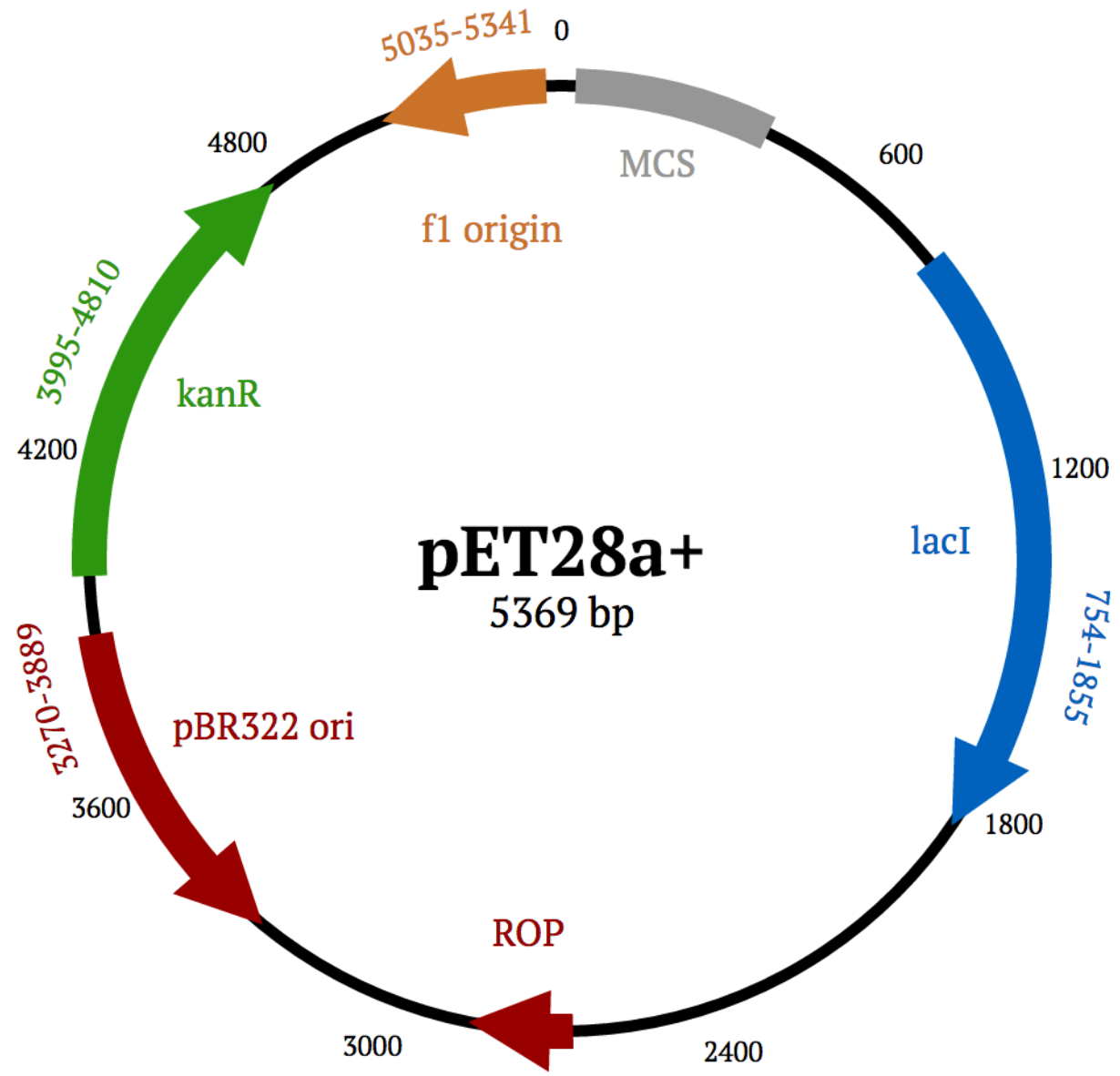

Figure 2-6 Plasmid map of expression vector pET28a+, used for His ${ }_{6}$-tag protein expression and expression of multi-genomic DNA in this study. Numbers denote the base pair location of genes of interest. Key features of the plasmid include the kanamycin (Kan) resistance cassettes (green), f1 origin (orange), lacI repressor (blue), MCS (grey, includes promoter, operator and ribosome binding sites, shown in greater detail in Figure 2-7), pBR322 ori and ROP regulatory protein (red). 
AAATTATACGACTCACTATAGGGGATTGTAGCGGATAACAATTCCCCTCTAGAAATATTTGTTTACTTTAAGAAGGAGATATCCATGG

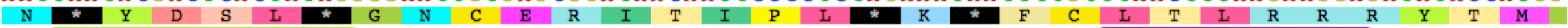
T7 promoter

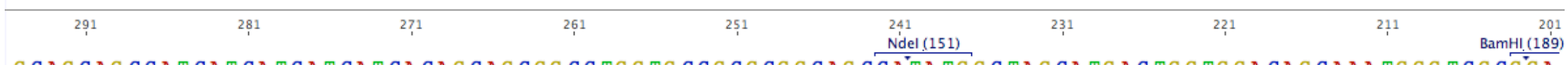

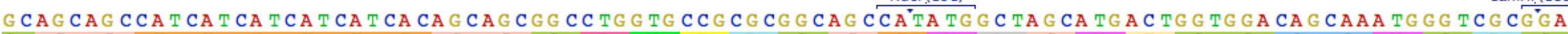

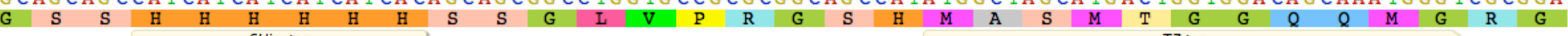
6 His-tag $\quad$ T7 tag

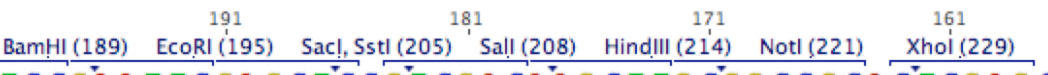

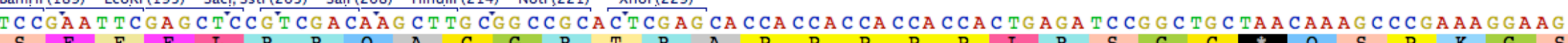

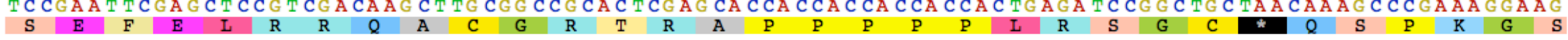

101 91

81

31

CTGAGTTGGCTGCTGCCACCGCTGAGCAATAACTAGCATAACCCTTGGGGCCTCTAAACGGTCTTGAGGGTTTTTGCTGAAAGAGGA
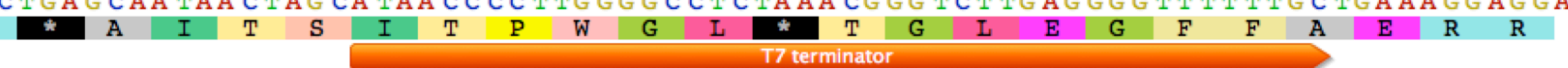

Figure 2-7 Multiple-cloning site of expression vector pET28a+. Numbers denote the base pair location of sequences of interest. Key features of the MCS include the T7 promoter binding site (light green), lac operator (red), rbs (purple), His $6_{-}$and T7 tags (yellow), T7 terminator (orange), and restriction enzyme sites. All restriction enzyme sites listed are unique to the multiple-cloning site. 


\subsection{Bacterial growth and maintenance}

\subsubsection{Growth media}

Unless otherwise stated, all media were made up in $\mathrm{ddH}_{2} \mathrm{O}$ and sterilised by autoclaving at $121^{\circ} \mathrm{C}$ for $30 \mathrm{~min}$, and stored at room temperature.

\subsubsection{Liquid media}

Table 2-5 Liquid media recipes

\begin{tabular}{|c|c|c|}
\hline Lysogeny broth (LB) ${ }^{a, b}$ & $\begin{array}{l}\text { Superoptimal broth } \\
\left(\text { SOB) }(-)^{b, c}\right.\end{array}$ & M9 minimal media \\
\hline Tryptone 10 g.L $\mathrm{L}^{-1}$ & Tryptone 20 g.L. ${ }^{-1}$ & 1x M9 salts (6.78 g...-1 \\
\hline Yeast extract 5 g. $\mathrm{L}^{-1}$ & Yeast extract $5 \mathrm{~g} \cdot \mathrm{L}^{-1}$ & $\mathrm{Na}_{2} \mathrm{HPO}_{4}, 3$ g.L. $\mathrm{L}^{-1} \mathrm{KH}_{2} \mathrm{PO}_{4}$, \\
\hline \multirow[t]{5}{*}{$\mathrm{NaCl} 5$ g.L $\mathrm{L}^{-1}$} & $\mathrm{NaCl} 10 \mathrm{mM}$ & $\begin{array}{l}1 \text { g. } \mathrm{L}^{-1} \mathrm{NH}_{4} \mathrm{Cl} \\
\left.0.5 \text { g. } \mathrm{L}^{-1} \mathrm{NaCl}\right)\end{array}$ \\
\hline & $\mathrm{KCl} 2.5 \mathrm{mM}$ & $2 \mathrm{mM} \mathrm{MgSO}_{4}{ }^{\mathrm{d}}$ \\
\hline & $\mathrm{MgCl}_{2} 10 \mathrm{mM}^{\mathrm{d}}$ & $100 \mu \mathrm{M} \mathrm{CaCl}{ }_{2}^{\mathrm{d}}$ \\
\hline & Glucose $20 \mathrm{mM}^{\mathrm{d}}$ & $0.4 \%$ glucose $^{\mathrm{d}}$ \\
\hline & $\mathrm{MgSO}_{4} 10 \mathrm{mM}^{\mathrm{d}}$ & \\
\hline
\end{tabular}

${ }^{a} \mathrm{LB}$ was provided as a premixed powder by manufacturer

${ }^{b}$ Adjusted to $\mathrm{pH} 7.0$ using $\mathrm{NaOH}$

'Superoptimal broth, when lacking Mg2+ ions and glucose, was denoted SOB (-

). After these extra reagents were added post-autoclave the media was renamed SOC.

${ }^{d}$ Filter-sterilized and added post-autoclave

\subsubsection{Solid media}

Agar plates for growth on solid media were made by addition of $1.5 \%(\mathrm{w} / \mathrm{v})$ agar to LB prior to autoclaving. Any necessary antibiotics were added post autoclave once the media had cooled to $<50^{\circ} \mathrm{C}$.

\subsubsection{Bacterial antibiotics and induction agents}

Antibiotics were made up in $\mathrm{diH}_{2} \mathrm{O}$ as stock solutions at 1000x final concentration, filter sterilised using a $0.22 \mu \mathrm{M}$ filter and stored in aliquots at $-20^{\circ} \mathrm{C}$. Final concentrations of antibiotics used to propagate plasmids in this study are described in Table 2-6. 
Table 2-6 Antibiotic final concentrations

\begin{tabular}{ll} 
Antibiotic & Final concentration \\
\hline Ampicillin (Amp) & $100 \mu \mathrm{g} \cdot \mathrm{mL}^{-1}$ \\
Spectinomycin (Spec) & $50 \mu \mathrm{g} \cdot \mathrm{mL}^{-1}$ \\
Kanamycin (Kan) & $50 \mu \mathrm{g} \cdot \mathrm{mL}^{-1}$ \\
Gentamicin (Gen) & $20 \mu \mathrm{g} \cdot \mathrm{mL}^{-1}$
\end{tabular}

Isopropyl $\beta$-D-1-thiogalactopyranoside (IPTG) for induction of genes from the lac operator was made up in $\mathrm{diH}_{2} \mathrm{O}$ to a concentration of $420 \mathrm{mM}(10 \%$ $\mathrm{w} / \mathrm{v}$ ), filter sterilised using a $0.22 \mu \mathrm{M}$ filter and stored in aliquots at $-20^{\circ} \mathrm{C}$.

\subsubsection{Growth and storage}

Unless otherwise stated, bacteria were grown at $37^{\circ} \mathrm{C}$, in a static incubator on solid media or at $200 \mathrm{rpm}$ in liquid media. For short term storage, strains were kept at $4{ }^{\circ} \mathrm{C}$ on solid agar plates. For long term storage, liquid cultures were mixed 1:1 with autoclaved $80 \%(\mathrm{v} / \mathrm{v})$ glycerol and stored indefinitely at $-80^{\circ} \mathrm{C}$.

\subsection{Standard molecular biology protocols}

\subsubsection{Isolation of plasmid DNA}

\subsubsection{Miniprep}

Preparation of plasmid DNA was carried out using the High-Speed Plasmid Mini Kit (Geneaid Biotech Ltd; New Taipei City, Taiwan) according to the manufacturer's instructions.

\subsubsection{Midiprep}

DNA midipreps were carried out using the Qiagen Plasmid Midi Kit (Qiagen N.V., Hilden, Germany) according to the manufacturer's instructions. 


\subsubsection{Polymerase chain reaction (PCR)}

Where PCR products were to be used in downstream cloning applications, amplification was carried out using Phusion ${ }^{\mathrm{TM}}$ high-fidelity polymerase. For colony screening and diagnostic PCR purposes Biomix Red ${ }^{\mathrm{TM}}$ was used. For the creation of error-prone libraries we used GeneMorph ${ }^{\circledR}$ II. PCR components and standard amplification protocols are shown below. Protocols for overlap PCR are described in Section 2.9.

\subsubsection{PCR components and protocol using Biomix Red}

For colony screens and diagnostic PCRs, a $10 \mu \mathrm{L}$ reaction consisting of $1 \mathrm{x}$ Biomix Red ${ }^{\mathrm{TM}}, 10$ - 50 ng plasmid DNA (or toothpick stab of a colony) and $0.5 \mu \mathrm{M}$ forward and reverse primers was used. The standard PCR protocol used is shown in Table 2-7.

Table 2-7 PCR protocol for Biomix Red

\begin{tabular}{ll|l} 
Temperature & Time & \\
\hline $98{ }^{\circ} \mathrm{C}$ & $3 \mathrm{~min}$ & \\
$95{ }^{\circ} \mathrm{C}$ & $30 \mathrm{~s}$ & 30 cycles \\
$\mathrm{T}_{\mathrm{A}}^{\mathrm{a}}$ & $30 \mathrm{~s}$ & \\
$72{ }^{\circ} \mathrm{C}$ & $30 \mathrm{~s}$ per kb DNA & \\
$72{ }^{\circ} \mathrm{C}$ & $10 \mathrm{~min}$ & \\
\hline${ }^{a} T_{\mathrm{A}} 2{ }^{\circ} \mathrm{C}$ below lowest $T_{M}$ of primers &
\end{tabular}

\subsubsection{PCR components and protocol using Phusion ${ }^{\mathrm{TM}}$}

For high-fidelity PCRs, a 20 - $200 \mu \mathrm{L}$ PCR using Phusion ${ }^{\mathrm{TM}}$ polymerase was used. Use of the HF buffer was preferred; however, if there were difficulties generating a PCR product, a switch to the GC buffer (which provides an error rate of $9.5 \times 10^{-7}$, compared to an error rate of $4.4 \times 10^{-7}$ for HF buffer) was used. All gene products amplified by Phusion ${ }^{\mathrm{TM}}$ PCR and cloned into a plasmid were sequence verified before use in downstream applications. PCR components and protocol are detailed in Table 2-8. 
Table 2-8 PCR components and protocol for Phusion ${ }^{\mathrm{TM}}$

\begin{tabular}{|c|c|c|c|}
\hline PCR component & $\begin{array}{l}\text { Final } \\
\text { concentration }\end{array}$ & $\begin{array}{l}\text { PCR } \\
\text { temperature }\end{array}$ & Time \\
\hline DNA template & $1-25 n g$ & $98^{\circ} \mathrm{C}$ & $30 \mathrm{~s}$ \\
\hline $\begin{array}{l}\text { Phusion }^{\mathrm{TM}} \\
\text { polymerase }\end{array}$ & $1 \mathrm{U} / 50 \mu \mathrm{L} \mathrm{PCR}$ & $98^{\circ} \mathrm{C}$ & $10 \mathrm{~s}$ \\
\hline Forward primer & $0.5 \mu \mathrm{M}$ & $\mathrm{T}_{\mathrm{A}}^{\mathrm{a}}$ & $20 \mathrm{~s}$ \\
\hline Reverse primer & $0.5 \mu \mathrm{M}$ & $72^{\circ} \mathrm{C}$ & $20 \mathrm{~s}$ per kb DNA \\
\hline $\begin{array}{l}5 x \text { HF or GC } \\
\text { buffer }\end{array}$ & $1 \mathrm{x}$ & $72{ }^{\circ} \mathrm{C}$ & $10 \mathrm{~min}$ \\
\hline DMSO & $3 \%$ & & \\
\hline dNTPs & $200 \mu \mathrm{M}$ & & \\
\hline $\mathrm{diH}_{2} \mathrm{O}$ & To volume & & \\
\hline
\end{tabular}

\subsubsection{PCR components and protocol using GeneMorph @ II}

For creation of error-prone gene libraries, the GeneMorph ${ }^{\circledR}$ II Random Mutagenesis Kit was used, which is formulated to provide minimal mutational bias in its libraries. Error rate is controlled by the amount of initial template DNA added to the PCR, and for most libraries generated we used approximately $10 \mathrm{ng}$ starting template and $30-40$ cycles, which gives 1 - 10 mutations per gene. PCR components and protocol are listed in Table 2-9.

Table 2-9 PCR components and thermocycling parameters for GeneMorph @ II

\begin{tabular}{|c|c|c|c|c|}
\hline PCR component & $\begin{array}{l}\text { Final } \\
\text { concentration }\end{array}$ & $\begin{array}{l}\text { PCR } \\
\text { temperature }\end{array}$ & Time & \multirow{8}{*}{$\begin{array}{l}30- \\
10,000 \\
\text { cycles }\end{array}$} \\
\hline DNA template & $1-10 \mathrm{ng}$ & $95^{\circ} \mathrm{C}$ & $2 \min$ & \\
\hline $\begin{array}{l}\text { Mutazyme II } \\
\text { polymerase }\end{array}$ & $\begin{array}{l}2.5 \mathrm{U} / 50 \mu \mathrm{L} \\
\text { PCR }\end{array}$ & $95^{\circ} \mathrm{C}$ & $30 \mathrm{~s}$ & \\
\hline Forward primer & $250 \mathrm{nM}$ & $\mathrm{T}_{\mathrm{A}}^{\mathrm{a}}$ & $30 \mathrm{~s}$ & \\
\hline Reverse primer & $250 \mathrm{nM}$ & $72^{\circ} \mathrm{C}$ & 1 min per kb DNA & \\
\hline $\begin{array}{l}\text { 10x Mutazyme II } \\
\text { buffer }\end{array}$ & $1 \mathrm{x}$ & $72^{\circ} \mathrm{C}$ & $10 \mathrm{~min}$ & \\
\hline dNTPs & $800 \mu \mathrm{M}$ & & & \\
\hline $\mathrm{diH}_{2} \mathrm{O}$ & To volume & & & \\
\hline
\end{tabular}




\subsubsection{Agarose gel electrophoresis}

When analysis of DNA samples was necessary, e.g. for examining results of PCR reactions or restriction digests, aliquots of DNA were mixed 6:1 with $6 \mathrm{x}$ DNA loading dye (30\% (v/v) glycerol, $0.25 \%(\mathrm{w} / \mathrm{v})$ bromophenol blue) where necessary and run on agarose gels (1\% w/v agarose in 1x TAE buffer $(40 \mathrm{mM}$ Tris-acetate, 1 mM EDTA, pH 8.3), $1 \mu \mathrm{g} . \mathrm{mL}^{-1}$ ethidium bromide) covered with 1x TAE in a suitable horizontal electrophoresis tank at 120-140 V for 20-40 min. DNA was visualised under UV light and size determined by comparison to Hyperladder I (Bioline, London, UK).

\subsubsection{DNA quantification}

Where required, purity and concentration of DNA products were determined using a NanoDrop ND-1000 spectrophotometer (Thermo Fisher Scientific; Waltham, MA, USA), according to the manufacturer's instructions.

\subsubsection{DNA product purification}

DNA products were purified using the DNA Clean and Concentrator ${ }^{\mathrm{TM}}-5 \mathrm{Kit}$ (Zymo Research; Irvine, CA, USA) according to manufacturer's instructions. DNA products that required purification included PCR products and DNA following restriction digests for cloning reactions. Following purification the DNA concentration was determined as per Section 2.6.4.

\subsubsection{Restriction enzyme digest}

When restriction digests were required for standard gene cloning and diagnostic purposes, digests were carried out as per manufacturer's instructions and heat inactivated if possible, then 50-200 ng of DNA was run on an agarose gel as per Section 2.6.3 to confirm successful cleavage. If digests were to be used in downstream cloning reactions, they were purified using DNA Clean and Concentrator ${ }^{\mathrm{TM}}-5$ columns (Section 2.6.5). 


\subsubsection{Restriction digest for mutant gene libraries}

For cloning of mutant gene libraries, which ideally required $>85 \%$ of plasmids to carry the mutant gene insert, certain modifications to restriction digest protocols were made. Prior to digestion, the vector was heated to 70 ${ }^{\circ} \mathrm{C}$ for 10 minutes before cooling to $37^{\circ} \mathrm{C}$ to relieve any backbone supercoiling and allow restriction enzymes unimpeded access to the restriction sites. Vector and insert were digested to the upper limit of recommended time according to manufacturers' instructions. When digesting a vector where a small region of DNA (> $100 \mathrm{bp}$ ) from the MCS was 'dropped out' following double restriction enzyme cleavage, the vector was purified as per Section 2.6.5 using a Geneaid miniprep column to capture the vector DNA, and the drop-out would not be bound due to its much smaller negative charge. Digested inserts were cleaned up on a DNA Clean and Concentrator ${ }^{\mathrm{TM}}-5$ column as per Section 2.6.5. DNA was stored in single use aliquots at $-20^{\circ} \mathrm{C}$ (to minimise free-thaw cycles) or used immediately in ligation protocols.

\subsubsection{Ligation}

Invitrogen T4 DNA Ligase was used for ligation reactions as shown in Table 2-10. Ligations were incubated overnight at $16^{\circ} \mathrm{C}$ before being used to transform cells.

Table 2-10 Ligation protocol with T4 DNA ligase

Component Final concentration

\begin{tabular}{ll}
\hline Linear vector DNA & $>100 \mathrm{ng}$ \\
Insert & $\begin{array}{l}\text { For small inserts: } 6: 1 \text { molar ratio over vector } \\
\text { For large inserts: } 3: 1 \text { molar ratio over vector }\end{array}$ \\
10x ligase buffer & To 1x final concentration \\
Ligase $\left(1 \mathrm{U} \cdot \mu \mathrm{L}^{-1}\right)$ & $1 \mu \mathrm{L}$ per $200 \mathrm{ng}$ total DNA \\
$\operatorname{diH}_{2} \mathrm{O}$ & To make to $20 \mu \mathrm{L}$ total volume per $100 \mathrm{ng}$ total \\
& DNA
\end{tabular}




\subsubsection{DNA sequencing}

Sequencing was carried out by Macrogen Inc. (Seoul, South Korea) to determine the sequence of any mutants of interest and verify correct insertion and sequence of every gene post insertion into a new vector backbone.

\subsubsection{Transformation of competent $E$. coli}

\subsubsection{Preparation of chemically competent cells}

An overnight culture of the strain to be made competent was grown in $2 \mathrm{ml}$ LB amended with any relevant antibiotics at $37^{\circ} \mathrm{C}, 200 \mathrm{rpm}$ overnight. The overnight culture was diluted 100-fold into a 20-50 mL flask of LB and the culture was grown at $37^{\circ} \mathrm{C}, 200 \mathrm{rpm}$ until an $\mathrm{OD}_{600}$ of 0.4-0.6 was reached. The culture was then placed on ice for 10 minutes in a $50 \mathrm{~mL}$ centrifuge tube, spun down at $4000 \mathrm{rpm} 4{ }^{\circ} \mathrm{C}$, for $10 \mathrm{~min}$, the supernatant removed and the cell pellet resuspended in $0.1 \mathrm{x}$ volume of ice cold TSS buffer (Table 2-11). Aliquots of the cells were then transferred into ice cold microcentrifuge tubes and either immediately transformed with plasmid DNA (Section 2.6.9.2) or snap frozen and stored at $-80^{\circ} \mathrm{C}$ until required.

Table 2-11 TSS Buffer recipe

TSS buffer

PEG 8000 100g.L -1 $^{-1}$

$\mathrm{MgCl}_{2} 30 \mathrm{mM}$

DMSO 0.05\% (v/v)

LB to make to volume

\subsubsection{Transformation of chemically competent cells}

Frozen aliquots of cells to be transformed were removed from $-80^{\circ} \mathrm{C}$ storage and fully defrosted on ice. DNA to be transformed into the cells was added and the mix left on ice for $30 \mathrm{~min}$. The cells were then heat shocked by incubation in a $42{ }^{\circ} \mathrm{C}$ heat block for $45 \mathrm{~s}$ and placed back on ice for 3-5 min. 
$100-200 \mu \mathrm{L}$ of LB or SOC was added to the cell mix and the cells allowed to recover at $37^{\circ} \mathrm{C}, 200 \mathrm{rpm}$ for $60 \mathrm{~min}$. Appropriate dilutions of recovered cells were then spread onto an agar plate containing the appropriate antibiotics. Plates were incubated overnight at $37^{\circ} \mathrm{C}$.

\subsubsection{Preparation of electrocompetent cells}

An LB agar plate (containing antibiotics where relevant) was streaked with the strain to be made competent and incubated overnight at $37^{\circ} \mathrm{C}$. A single colony from this agar plate was used to inoculate $4 \mathrm{~mL}$ of LB overnight at 37 ${ }^{\circ} \mathrm{C}, 200 \mathrm{rpm}$. This overnight was transferred to a $2.5 \mathrm{~L}$ flask containing 400 $\mathrm{mL} \mathrm{SOB}(-)$ pre-warmed to $37^{\circ} \mathrm{C}$ and appropriate antibiotics and the resulting culture was grown at $37^{\circ} \mathrm{C}, 200 \mathrm{rpm}$ until an OD600 of $0.4-0.7$ was reached, at which point the culture was transferred into eight $50 \mathrm{ml}$ centrifuge tubes and placed in an ice/water bath for 15-30 minutes with occasional swirling. The cells were then collected by centrifugation (4000 rpm, $10 \mathrm{~min}, 4^{\circ} \mathrm{C}$ ), supernatant discarded and the pellets resuspended in a total volume of $400 \mathrm{~mL}$ ice cold sterile $\mathrm{diH}_{2} \mathrm{O}$ by gentle pipetting. The cells were again collected by centrifugation $\left(4000 \mathrm{rpm}, 10 \mathrm{~min}, 4^{\circ} \mathrm{C}\right)$ and each resulting pellet resuspended in $25 \mathrm{ml}$ ice-cold sterile $10 \%(\mathrm{v} / \mathrm{v})$ glycerol. The cells were pelleted by centrifugation $\left(4000 \mathrm{rpm}, 15 \mathrm{~min}, 4^{\circ} \mathrm{C}\right)$ and all the resulting pellets resuspended in a total volume of $15 \mathrm{ml}$ ice cold sterile $10 \%$ v/v glycerol and placed into one $15 \mathrm{~mL}$ centrifuge tube. The cells were subjected to a final centrifugation $\left(4000 \mathrm{rpm}, 15 \mathrm{~min}, 4^{\circ} \mathrm{C}\right.$ ) and the supernatant fully discarded. The pellet was resuspended in as little 10\% glycerol as needed to form a liquid suspension of cells, resulting in a 200$500 \mu \mathrm{L}$ volume of cells. $40 \mu \mathrm{L}$ aliquots were distributed into pre-chilled 1.5 $\mathrm{mL}$ microcentrifuge tubes on ice and snap-frozen using a metal tube block cooled to $-80{ }^{\circ} \mathrm{C}$, and stored at $-80^{\circ} \mathrm{C}$ until needed. 


\subsubsection{Transformation of electrocompetent cells}

Aliquots of frozen cells $(40 \mu \mathrm{L})$ were defrosted on ice. DNA to be electroporated ( 0.2 to $0.5 \mu \mathrm{L}$ of a ligation, and up to $10 \mathrm{ng}$ of a purified plasmid) was diluted out to $5 \mu \mathrm{L}$ with $\mathrm{diH}_{2} \mathrm{O}$, then added to the cells and gently mixed. The cells were then moved to $0.2 \mathrm{~mm}$ gap electroporation cuvettes pre-chilled on ice and electroporated using a Bio-Rad GenePulser Xcell $^{\mathrm{TM}}$ (Hercules, CA, USA). The cells were pulsed $(2.5 \mathrm{kV}, 25 \mu \mathrm{F}, 200 \Omega)$ and 2 sequential steps of $750 \mu \mathrm{L}$ room temperature SOC added immediately and the cell resuspension transferred to a $15 \mathrm{~mL}$ centrifuge tube for recovery. For transformation of a ligation where merely a screenable number of colonies was required, cells were allowed to recover for 60 minutes at $37^{\circ} \mathrm{C}, 200 \mathrm{rpm}$, after which multiple dilutions were plated on agar plates containing the appropriate antibiotic. For transformation of a library, the post-recovery protocol is detailed in Section 2.6.9.5.

\subsubsection{Recovery of electrocompetent cells transformed with library DNA}

Following recovery of transformed cells in SOC for $60 \mathrm{~min}$, all transformations except for the negative control were combined in a $50 \mathrm{~mL}$ centrifuge tube, and $0.01 \mu \mathrm{L}, 0.1 \mu \mathrm{L}, 1 \mu \mathrm{L}$ and $10 \mu \mathrm{L}$ plated in triplicate on $\mathrm{LB}$ agar plates containing the appropriate antibiotic and incubated overnight at $37^{\circ} \mathrm{C}$. The rest of the library was transferred to a suitable sized flask (with a 5 -fold larger volume than the total final volume of growth media) containing $20 \mathrm{~mL} \mathrm{LB}$ per transformation and relevant antibiotics and recovered at $37^{\circ} \mathrm{C}$, $200 \mathrm{rpm}$ for $6 \mathrm{~h}$, then spun in $50 \mathrm{~mL}$ centrifuge tubes at $4000 \mathrm{rpm}, 4{ }^{\circ} \mathrm{C}$ for 10 min. The supernatant was discarded and each cell pellet resuspended in 100 - $200 \mu \mathrm{L} \mathrm{LB}$ and the cells combined in a $15 \mathrm{~mL}$ centrifuge tube. An $\mathrm{OD}_{600}$ reading of $5-10 \mu \mathrm{L}$ of resuspended cells was taken to determine the fold coverage of the library in the resuspension (an $\mathrm{OD}_{600}$ of 1.0 was determined to contain on average $1 \times 10^{9}$ cells), with a count of colonies on agar plates used to determine the transformed library size. 


\subsection{Nitroreductase candidate library}

The bacterial oxidoreductase enzymes tested for activity with various nitroaromatic substrates are shown in Column 1 of Table 2-12. Each had been individually PCR amplified from gDNA preparations of indicated bacterial species and cloned using restriction digest into the pUCX expression vector before transformation into specialised $E$. coli assay strains by previous members of the Ackerley lab (Prosser et al., 2013; Williams, 2013). To distinguish genes or enzymes having the same family name, each oxidoreductase was referred to using standard nomenclature followed by a two-letter abbreviation from the genus and species of bacteria the enzyme originated from. Enzymes are colour-coded by family (Section 2.7.1). Five additional nitroreductases (YcnD_Bli, NfrA_Bli, YfkO_Bli, NfsB_Nme, YwrO_Bam) were added to the library over the course of this thesis to replace nitroreductases that had little activity with nitroaromatic substrates of interest in the core 58 nitroreductase pool (NQO1_Pp, NQO1_Pa, YdjA_Kp, YwrO_Vf, KefF_Ec). These five replacement nitroreductases were ordered as synthetic genes (gBlocks) from Integrated DNA Technologies (IDT; Coralville, IA, USA) with restriction enzyme cleavage sites at either end of the gene, and cloned using restriction digest into the pUCX expression vector before transformation into specialised $E$. coli assay strains. 
Table 2-12 Oxidoreductase enzymes in the expanded nitroreductase candidate library

\begin{tabular}{|c|c|c|c|c|}
\hline Gene & Organism & Family & Superfamily & Accession number \\
\hline NfsA_Ec & Escherichia coli & NfsA & Nitro FMN reductase & $\underline{\mathrm{APC} 51123.1}$ \\
\hline NfsA_St & Salmonella enterica serovar Typhimurium & NfsA & Nitro FMN reductase & WP 000075306.1 \\
\hline NfsA_Ck & Citrobacter koseri & NfsA & Nitro FMN reductase & WP 012133106.1 \\
\hline NfsA_Kp & Klebsiella pneumoniae & NfsA & Nitro FMN reductase & WP 004179133.1 \\
\hline NfsA_Es & Cronobacter sakazakii & NfsA & Nitro FMN reductase & WP 007848889.1 \\
\hline NfsA_Eca & $\begin{array}{l}\text { Pectobacterium atrosepticum } \\
\text { (previously Erwinia carotovora) }\end{array}$ & NfsA & Nitro FMN reductase & WP 039295639.1 \\
\hline NfsA_Vf & $\begin{array}{l}\text { Aliivibrio fischeri (previously Vibrio } \\
\text { fischeri) }\end{array}$ & NfsA & Nitro FMN reductase & WP 054775176.1 \\
\hline NfsA_Vv & Vibrio vulnificus & NfsA & Nitro FMN reductase & WP 017421853.1 \\
\hline Frp_Vh & Vibrio harveyi & NfsA & Nitro FMN reductase & WP 038898257.1 \\
\hline CO-Frp_Vh & Vibrio harveyi & NfsA & Nitro FMN reductase & $\underline{\mathrm{Q} 56691.1}$ \\
\hline NfrA_Bs & Bacillus subtilis & NfsA & Nitro FMN reductase & WP 003222161.1 \\
\hline NfsA_Lw & Listeria welshimeri & NfsA & Nitro FMN reductase & $\overline{\text { WP } 011701746.1}$ \\
\hline NfsA_Li & Listeria innocua & NfsA & Nitro FMN reductase & WP 003761397.1 \\
\hline NfsA_Bc & Bacillus coagulans & NfsA & Nitro FMN reductase & WP 029141841.1 \\
\hline NfsA_Np & Nostoc punctiforme & NfsA & Nitro FMN reductase & $\underline{\mathrm{ACC} 79374.1}$ \\
\hline NfsA_Bt & Bacillus thuringiensis & NfsA & Nitro FMN reductase & WP 070179995.1 \\
\hline NfsA_Ls & Lactobacillus sakei & NfsA & Nitro FMN reductase & WP 035146643.1 \\
\hline EcD_Pp & Pseudomonas putida & NfsA & Nitro FMN reductase & WP 010953425.1 \\
\hline YcnD_Bs & Bacillus subtilis & NfsA & Nitro FMN reductase & WP 003225013.1 \\
\hline NfsA_Ms & Mycobacterium smegmatis & NfsA & Nitro FMN reductase & WP 011728689.1 \\
\hline YcnD_Bli & Bacillus licheniformis & NfsA & Nitro FMN reductase & WP 003178951.1 \\
\hline NfrA_Bli & Bacillus licheniformis & NfsA & Nitro FMN reductase & WP 003186165.1 \\
\hline NfsB_Ec & Escherichia coli & NfsB & Nitro FMN reductase & CQR80176.1 \\
\hline
\end{tabular}


Gene

NfsB_Ck

NfsB_St

NfsB_Kp

NfsB_Vv

NfsB_Pp

NfsB_Es

FraseI_Vf

NfsB_Vh

YfkO_Bs

YfkO_Bli

NfsB_Nme

YdgI_Bs

NfsB_Pa

AzoR Ec

AzoR_St

AzoR_Vv

AzoR Pp

NemA_Ec

NemA_St

NemA Kp

NemA_Vv

YwrO_Bs

YwrO_Li

YwrO_Bam

YwrO Vf

YieF_Ec

YieF_Pa
Organism

Citrobacter koseri

Salmonella enterica

Klebsiella pneumoniae

Vibrio vulnificus

Pseudomonas putida

Cronobacter sakazakii

Aliivibrio fischeri

Vibrio harveyi

Bacillus subtilis

Bacillus licheniformis

Neisseria meningitidis

Bacillus subtilis

Pseudomonas aeruginosa

Escherichia coli

Salmonella enterica

Vibrio vulnificus

Pseudomonas putida

Escherichia coli

Salmonella enterica

Klebsiella pneumoniae

Vibrio vulnificus

Bacillus subtilis

Listeria innocua

Bacillus amyloliquefaciens

Aliivibrio fischeri

Escherichia coli

Pseudomonas aeruginosa
Family

NfsB

NfsB

NfsB

$\mathrm{NfsB}$

$\mathrm{NfsB}$

$\mathrm{NfsB}$

$\mathrm{NfsB}$

$\mathrm{NfsB}$

$\mathrm{NfsB}$

$\mathrm{NfsB}$

NfsB

Nitroreductase 1

Nitroreductase 1

AzoR

AzoR

AzoR

AzoR

OYE-like FMN

OYE-like FMN

OYE-like FMN

OYE-like FMN

$\mathrm{MdaB}$

$\mathrm{MdaB}$

$\mathrm{MdaB}$

$\mathrm{MdaB}$

SsuE

SsuE
Superfamily Nitro FMN reductase Nitro FMN reductase

Nitro FMN reductase Nitro FMN reductase

Nitro FMN reductase Nitro FMN reductase

Nitro FMN reductase Nitro FMN reductase Nitro FMN reductase Nitro FMN reductase Nitro FMN reductase Nitro FMN reductase Nitro FMN reductase FMN reductase FMN reductase FMN reductase FMN reductase TIM phosphate binding TIM phosphate binding TIM phosphate binding TIM phosphate binding FMN reductase FMN reductase FMN reductase FMN reductase

FMN reductase

FMN reductase
Accession number WP 047458272.1 WP 000355874.1

WP 004178896.1 WP 017421465.1

WP 010953384.1 WP 004384956.1

P46072.3

WP 005435698.1

ADE73858.1

WP 003179779.1

WP 002225416.1

WP 003225379.1

WP 003100063.1

WP 000048950.1

WP 000048924.1

WP 017421098.1

ABQ77513.1

WP 000093589.1

WP 000092935.1

WP 004148597.1

WP 017421369.1

WP 003219573.1

WP 003772432.1

WP 014471115.1

WP 012534890.1

WP 001291268.1

WP 003082483.1 


\begin{tabular}{lllll} 
Gene & Organism & Family & Superfamily & Accession number \\
\hline MdaB_Ec & Escherichia coli & MdaB & FMN reductase & $\underline{\text { WP_050864130.1 }}$ \\
MdaB_Ps & Pseudomonas syringae & MdaB & FMN reductase & $\underline{\text { WP 011167644.1 }}$ \\
WrbA_Ec & Escherichia coli & WrbA & FMN reductase & $\underline{\text { WP 001151437.1 }}$ \\
WrbA_Ps & Pseudomonas syringae & WrbA & FMN reductase & $\underline{\text { WP 004657185.1 }}$ \\
YdjA_Ec & Escherichia coli & Arsenite oxidase & Nitro FMN reductase & $\underline{\underline{E G I 21339.1}}$ \\
YdjA_Kp & Klebsiella pneumoniae & Arsenite oxidase & Nitro FMN reductase & $\underline{\text { EKF79369.1 }}$ \\
YcdI_Ec & Escherichia coli & Nitroreductase 5 & Nitro FMN reductase & $\underline{\text { WP 001001176.1 }}$ \\
YcdI_Kp & Klebsiella pneumoniae & Nitroreductase 5 & Nitro FMN reductase & $\underline{\text { WP_004141442.1 }}$ \\
YcaK_Ec & Escherichia coli & MdaB & FMN reductase & $\underline{\text { WP 001190363.1 }}$ \\
YcaK_Pa & Pseudomonas aeruginosa & MdaB & FMN reductase & $\underline{\text { WP 003114232.1 }}$ \\
NQO1_Pp & Pseudomonas putida & MdaB & FMN reductase & $\underline{\text { WP 010954556.1 }}$ \\
NQO1_Pa & Pseudomonas aeruginosa & MdaB & FMN reductase & $\underline{\text { WP 003102071.1 }}$ \\
KefF_Ec & Escherichia coli & MdaB & FMN reductase & $\underline{\text { ERA11253.1 }}$ \\
Empty & & & &
\end{tabular}




\subsubsection{Bioinformatics}

Accession numbers were found by entering the oxidoreductase protein sequence into the algorithm protein-protein BLAST (blastp;

http://blast.ncbi.nlm.nih.gov/), run by the National Centre for Biotechnology Information (NCBI). Family and superfamily information was obtained from BLAST results.

\subsubsection{Expanded nitroreductase family tree}

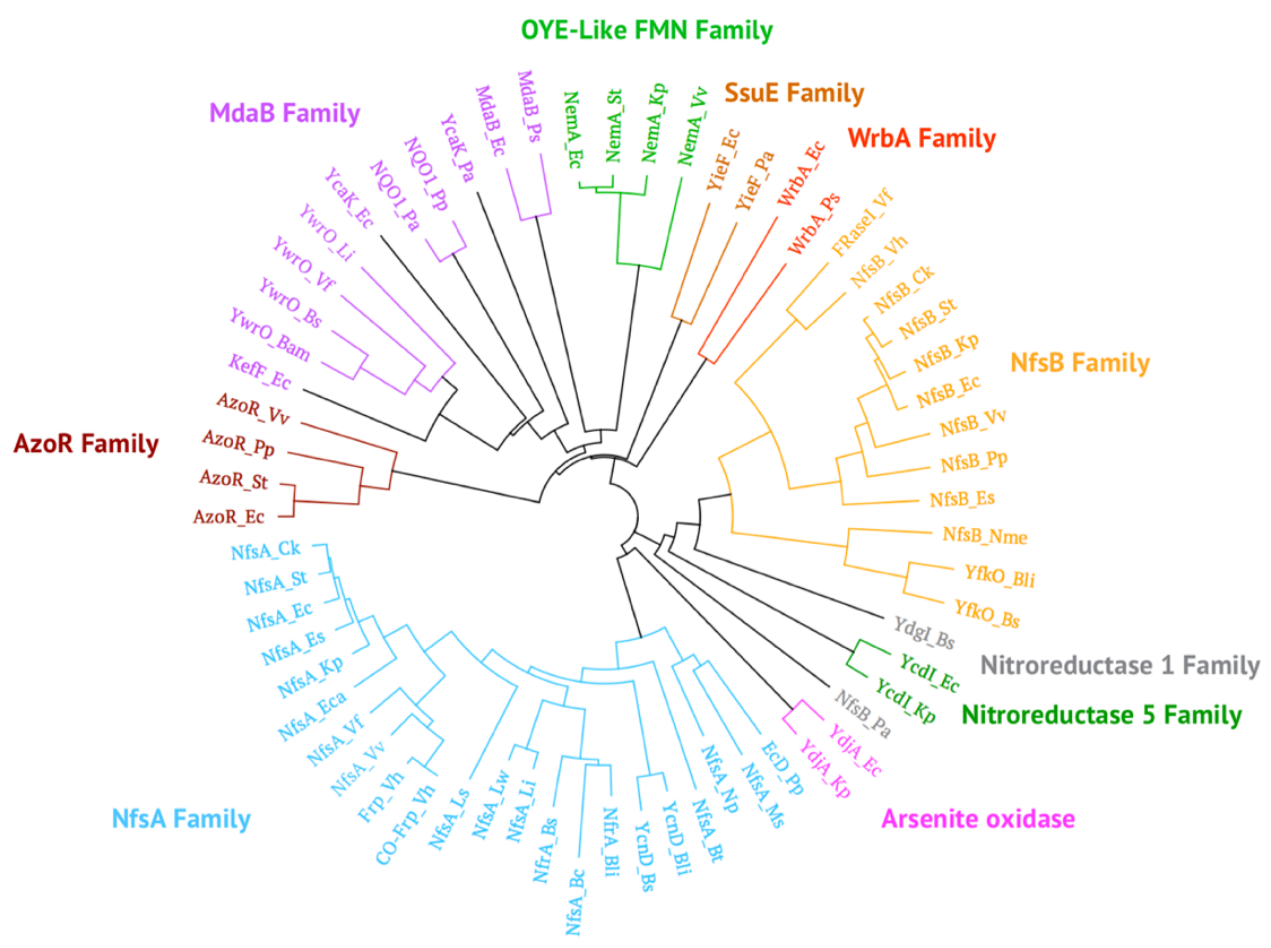

Figure 2-8 Phylogenetic tree of enzymes in the expanded nitroreductase candidate library. Further detail of enzymes in this tree can be found in Table 2-12. Enzymes were aligned by an identity cost matrix using Geneious 8.0, and are colour-coded by family (as defined by NCBI BLAST).

Geneious 8.0 (Biomatters Ltd; Auckland, NZ) was used to build a protein phylogenetic tree for the nitroreductases listed in Table 2-12, using a global alignment with free end gaps, an identity cost matrix, Jukes-Cantor genetic distance model and neighbour-joining tree build method. The phylogenetic tree (Figure 2-8) shows the enzymes clustered into 2 distinct arms: the first, 
consisting of enzymes from the Nitro FMN reductase superfamily, contains the members from the NfsA, NfsB, Nitroreductase 1 and 5, and Arsenite Oxidase families; the second consists of families located in the FMN reductase superfamily and the NemA enzyme family.

\subsection{NfsA_Ec 7SM site saturation mutagenesis library}

This library was designed by Drs Copp and Williams, and utilised the NDT degenerate codon $(\mathrm{N}$ : adenine, cytosine, guanine, thymine; $\mathrm{D}$ : adenine/guanine/thymine, $\mathrm{T}$ : thymine) at six selected active site residues (S41, F42, F83, S224, R225, F227) as it also allowed for the wild-type amino acid to be coded for at that site. For amino acid site K222, as wild-type Lys could not be coded for with NDT, the degenerate codon NNK (K: guanine/thymine) was utilised instead, which allows for 32 codons and all 20 proteinogenic amino acids (Reetz et al., 2008). The 7SM library had in total nearly 96 million possible codon combinations, and was ordered as a synthetic gene cloned into the vector pUCX:empty (low-expressing) by GenScript (Piscataway, New Jersey, USA), which guaranteed a total library size of 10 million unique variants. The lyophilised library was resuspended in $100 \mu \mathrm{L}$ TE and either used immediately in transformations or aliquoted into microcentrifuge tubes and frozen at $-20{ }^{\circ} \mathrm{C}$ indefinitely.

\subsection{Overlap PCR for targeted amino acid substitution of a single site residue}

Nitroreductase single site variants were created using overlap PCR as per Dr Williams' protocol which is summarised below (Figure 2-10; Williams et al., 2014). For each substitution to be made, two internal gene primers were designed. The forward internal primer was comprised of three parts, first a $\sim 18$ nucleotide section homologous to the gene section immediately upstream of the substitution site, next the actual mutant codon and finally $\sim 15$ nucleotides homologous to the gene region immediately after the 
substitution site. The reverse internal primer was the reverse complement of the first $\sim 18$ nucleotides of the forward internal primer. An example of such primers can be seen in Figure 2-9. A schematic of how such internal primers, in combination with flanking gene primers, can be used to generate a full-length gene using overlap PCR can be seen in Figure 2-10. The initial gene fragments were amplified in separate PCR reactions from a pUCX: wild type nitroreductase gene template ( $500 \mathrm{pg}$ of plasmid per reaction), using a standard Phusion ${ }^{\mathrm{TM}}$ protocol (Section 2.6.2.2). PCR products were then purified, combined in a 1:1 molar ratio and a full length gene product amplified using an overlap PCR protocol (Figure 2-10). Note that for targeted residues that occurred within 45 nucleotides from either the start or the end of the gene, a longer flanking gene primer which contained the desired codon change, combined with the standard reverse gene flanking primer, was all that was required, and a standard Phusion ${ }^{\mathrm{TM}}$ PCR reaction on the gene template (Section $\mathbf{2 . 6 . 2 . 2}$ ) was run using these two primers.

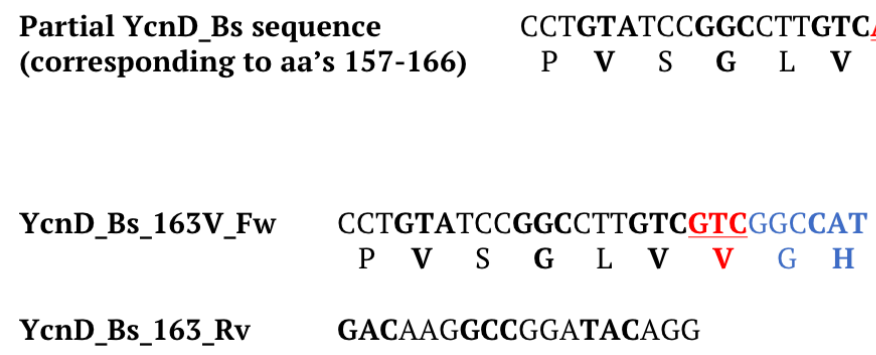

Figure 2-9 Example of primers used to generate site targeted mutants. Primers designed to generate the ycnD_Bs I163V mutant from wild-type ycnd_Bs. The site of amino acid substitution is marked in red and primer overlap region in blue. Primers are displayed in the 5'-3' direction. 

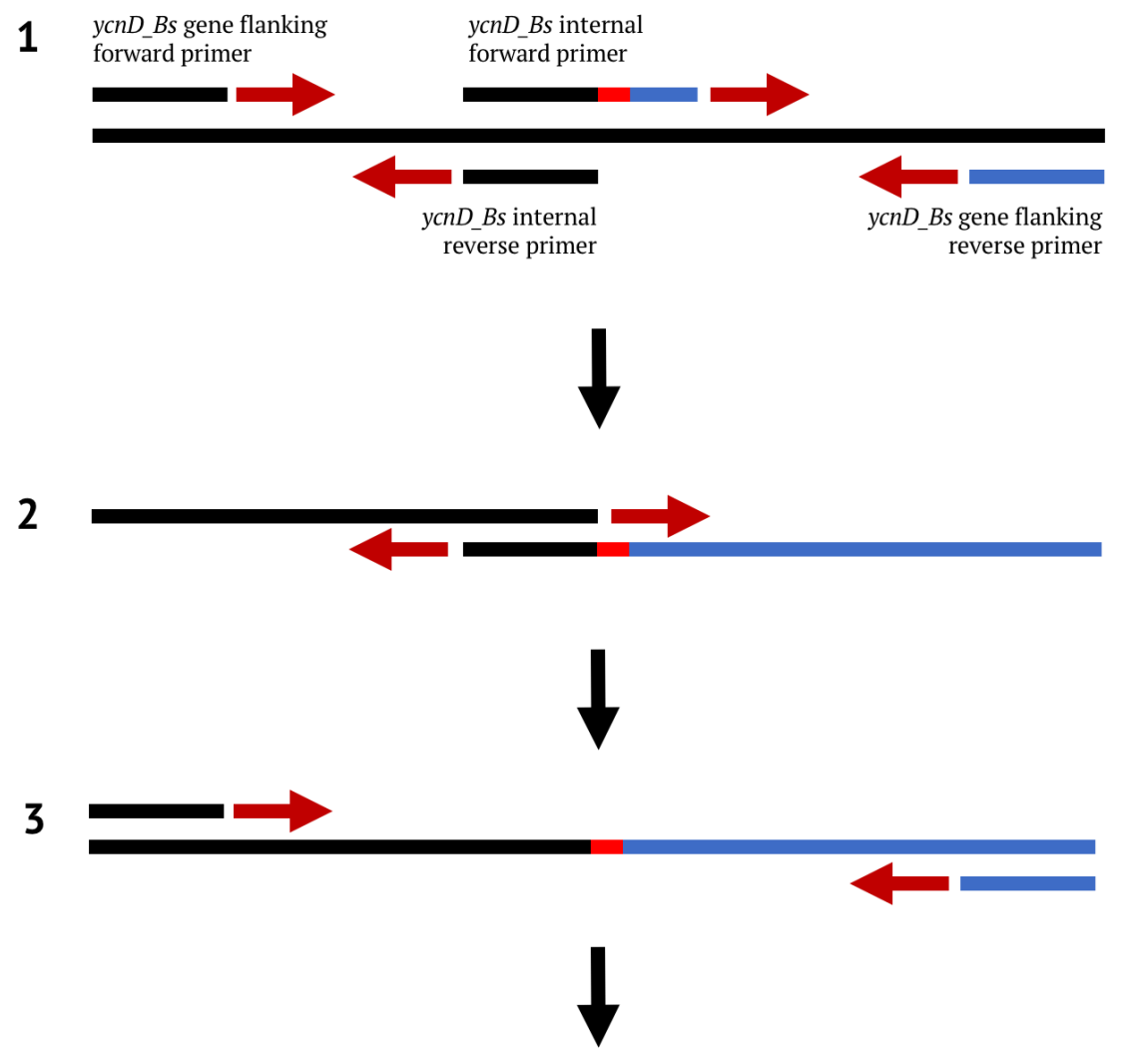

Figure 2-10 Schematic of overlap PCR for generation of site directed mutants.Two gene fragments are separately amplified using the wild type gene as template, with one internal primer and complementary gene specific primer in each reaction. The second fragment contains the amino acid substitution specified by the internal primer. 2) These fragments, which have a 18 base pair overlap due to the specific design of the internal primers are combined. A primer-less PCR reaction is commenced and due to this overlap region the fragments self-prime. 3) After a number of PCR cycles gene specific primers are added and increase amplification of the full-length gene, which now contains the specified amino acid substitution.

\subsection{Generation of error-prone nitroreductase libraries}

\subsubsection{PCR creation and cloning of error-prone libraries}

\subsubsection{Phusion ${ }^{T M}$ and Genemorph ${ }^{T M} / /$ PCR}

Genes that were to be used as template for an error-prone library were first amplified using Phusion ${ }^{\mathrm{TM}}$ polymerase as shown in Section 2.6.2.2.

Following DNA purification and quantification, the PCR product was used 
for an error-prone PCR as shown in Section 2.10.1. The PCR product was cleaned up using a DNA Clean and Concentrator ${ }^{\mathrm{TM}}-5$ Column, and restriction enzymes used to cleave both the PCR product and plasmid.

\subsubsection{Ligation}

Small scale ligations (approx. $100 \mathrm{ng}$ total DNA) were performed with multiple different insert:vector ratios (as per Section 2.6.7) and transformed into electrocompetent $E$. coli, and the resulting colonies PCR screened to determine the insert:vector ratio that generated the highest proportion of successfully ligated inserts. A random selection of colonies containing ligated inserts were then sequenced to confirm the desired mutation rate, after which a ligation was performed using the rest of the digested PCR and vector. The ligation was heat-inactivated and either stored at $4{ }^{\circ} \mathrm{C}$ for up to one month, or $5-10 \mu \mathrm{L}$ aliquoted into microcentrifuge tubes and stored at $20^{\circ} \mathrm{C}$. Care was taken to thaw each ligation only once prior to transformation.

\subsubsection{Transformation}

Electrocompetent $E$. coli cells were made according to the protocol in Section 2.6.9.3 and the ligation transformed in as per Section 2.6.9.4. For libraries where $<1 \times 10^{6}$ transformants were achieved upon transformation of the ligation into one aliquot of an $E$. coli screening strain, the ligation was first transformed into electrocompetent E. coli EC100 cells (made using the same protocol in Section 2.6.9.3), a highly competent strain for ligation transformations, and the cells recovered as per Section 2.6.9.5. A miniprep of the cells was made and the library transformed into the relevant E. coli screening strain. 


\subsection{Selection of nitroreductase libraries using positive selection compounds}

2.11.1 Niclosamide selection of nitroreductase libraries in SOS-R4 cell strain In SOS-R4 the transformed gene library, following 1 hour recovery in SOC, was plated directly on $150 \mathrm{~mm}$ petri dishes containing LB agar + Amp + Spec $+0.5 \mu \mathrm{M}$ niclosamide, with dilutions of the transformation plated on small plates with and without niclosamide to estimate library size and percentage niclosamide selection. Colonies that were present the following day on each $150 \mathrm{~mm}$ petri dish were resuspended in $2 \mathrm{~mL}$ LB using a glass spreader and transferred to a $50 \mathrm{~mL}$ centrifuge tube. The cells were spun at $4000 \mathrm{rpm}$ for 30 minutes and the resulting pellet resuspended in 5-10 mL LB and mixed $1: 1$ with $80 \%$ glycerol $(\mathrm{v} / \mathrm{v})$ and the $\mathrm{OD}_{600}$ noted. The resuspension was stored at $-80^{\circ} \mathrm{C}$ in microcentrifuge tubes. For flow cytometry experiments, enough of the resuspension to allow for at least 100 -fold coverage of the library was used as a starter for the overnight culture (see Section 2.15.2 for FACS protocol).

\subsubsection{Niclosamide or chloramphenicol selection of nitroreductase libraries in 7NT or 7TL cell strains}

7NT or 7TL cells transformed with the variant library were recovered as per Section 2.6.9.5, resuspended in 1:1 LB and 80\% (w/v) glycerol and stored as aliquots at $-80^{\circ} \mathrm{C}$. The resuspended library was plated on large LB agar + Amp plates containing concentrations of niclosamide that allowed for growth of colonies above that of which wild-type enzymes could grow. If issues with growth were observed then varying concentrations of IPTG, allowing for upregulation of niclosamide-detoxifying gene expression, were also used. These colonies were subjected to further screening with specific prodrugs using growth inhibition assays (Section 2.15.3). 


\subsection{Protein purification}

\subsubsection{Expression using low temperature LB + supplements}

Cells containing pET28a+:nitroreductase in the expression strain BL21(DE3) were used to inoculate a $1-4 \mathrm{~mL}$ culture of $\mathrm{LB}+$ Kan overnight at $37^{\circ} \mathrm{C}, 200$ rpm. The following day the overnight was diluted 100-fold into $50-100 \mathrm{~mL}$ fresh $\mathrm{LB}+$ Kan in a culture flask and incubated at $37^{\circ} \mathrm{C}, 200 \mathrm{rpm}$ until it reached an $\mathrm{OD}_{600}$ of $0.5-0.7$. The culture and flask were chilled on ice for 20 minutes, after which IPTG was added to a final concentration of $0.5 \mathrm{M}$. The flask was then incubated in a chilled incubator at $18{ }^{\circ} \mathrm{C}, 200 \mathrm{rpm}$ for $16-18$ hours. The culture was transferred to $50 \mathrm{~mL}$ centrifuge tubes and centrifuged at $4000 \mathrm{rpm}, 4^{\circ} \mathrm{C}$ for 15 minutes, after which the supernatant was discarded and the pellet was either frozen at $-80^{\circ} \mathrm{C}$ or processed immediately.

\subsubsection{Enzyme purification using $\mathrm{His}_{6}$-tag and Ni-NTA column}

Cell pellets containing His $_{6}$-tagged over-expressed protein were resuspended in $2.5 \mathrm{~mL}$ Bugbuster ${ }^{\circledR}$ per $50 \mathrm{~mL}$ induced culture. The cell resuspension was mixed gently on a shaking platform for $30 \mathrm{~min}$, following which the solution was transferred into $1.5 \mathrm{~mL}$ microcentrifuge tubes and placed on ice for $10 \mathrm{~min}$. The tubes were centrifuged at max speed $(>13,000$ $\mathrm{rpm}), 4^{\circ} \mathrm{C}$ for 30 minutes and the yellow soluble protein fractions pooled in a $15 \mathrm{~mL}$ centrifuge tube on ice. Purification of $\mathrm{His}_{6}$-tagged proteins from the soluble fraction was carried out using Ni-NTA His.Bind $\circledast$ Resin (Merck Milipore, MA, USA) and a peristaltic pump. Recipes for protein purification buffers used in the following protocol are listed in Table 2-13.

First, $2-4 \mathrm{~mL}$ of Ni-NTA resin was added to a Pierce $10 \mathrm{~mL}$ disposable column (Thermo Fisher Scientific, Waltham, MA, USA) connected to the peristaltic pump and excess liquid was left to flow through until the liquid level was just above the settled resin bed. $4 \mathrm{~mL}$ of $\mathrm{ddH}_{2} \mathrm{O}$ was added to the top of the resin and allowed to flow through until the liquid level was just above the resin bed again. $8 \mathrm{~mL}$ of Charge buffer was added to the column 
and allowed to flow through, followed by $6 \mathrm{~mL}$ of Bind buffer, which was allowed to flow through until liquid level was $1 \mathrm{~cm}$ above settled resin bed. The soluble protein fraction was added to the column and mixed with the remaining Bind buffer and resin by gentle pipetting, then pumped through, during which the column turned yellow from binding FMN-containing protein. Another $10 \mathrm{~mL}$ of Bind buffer was washed through the column, followed by $8 \mathrm{~mL}$ of Wash buffer, which was pumped through until liquid level was level with the top of the resin bed. $4 \mathrm{~mL}$ of Elute buffer was added to the column, and flow-through was collected in $500 \mu \mathrm{L}$ fractions until the resin bed started to run dry. $4 \mathrm{~mL}$ of Strip buffer was then run through the column in preparation for the next protein sample, and the protocol starting from the addition of $\mathrm{ddH}_{2} \mathrm{O}$ to the column repeated. If no more protein samples were to be purified, the resin beads were stored in Strip buffer at 4 ${ }^{\circ} \mathrm{C}$. For each eluted protein, the three most yellow fractions by eye were pooled and incubated with $1 \mathrm{mM}$ excess FMN cofactor for 1 hour on ice. Buffer-exchange into $40 \mathrm{mM}$ Tris- $\mathrm{Cl} \mathrm{pH} 7.0$ was then conducted using a $5 \mathrm{~mL}$ HiTrap $^{\mathrm{TM}}$ desalting column (GE Healthcare, Little Chalfont, UK) according to the manufacturer's instructions. Only $1.5 \mathrm{~mL}$ of column flow-through was collected upon elution to minimize potential contamination with excess free FMN. The purified protein was then mixed 1:1 with $80 \%$ glycerol prior to storage and determination of concentration. His $_{6}$-tags were not removed from purified proteins as tag presence had previously been determined to not affect the catalytic activity of purified nitroreductases (Prosser et al., 2013).

Table 2-13 His-tag purification buffer recipes

\begin{tabular}{lllll} 
Charge buffer & Bind buffer & Wash buffer & Elute buffer & Strip buffer \\
\hline $50 \mathrm{mM}$ & $500 \mathrm{mM}$ & $500 \mathrm{mM}$ & $500 \mathrm{mM}$ & $500 \mathrm{mM}$ \\
$\mathrm{NiSO}_{4}$ & $\mathrm{NaCl}$ & $\mathrm{NaCl}$ & $\mathrm{NaCl}$ & $\mathrm{NaCl}$ \\
& $20 \mathrm{mM}$ Tris- & $20 \mathrm{mM}$ Tris- & $20 \mathrm{mM}$ Tris- & $20 \mathrm{mM}$ Tris- \\
& $\mathrm{HCl} \mathrm{pH} 7.9$ & $\mathrm{HCl} \mathrm{pH} 7.9$ & $\mathrm{HCl} \mathrm{pH} 7.9$ & $\mathrm{HCl} \mathrm{pH} 7.9$ \\
& $5 \mathrm{mM}$ & $60 \mathrm{mM}$ & $1 \mathrm{M}$ & $100 \mathrm{mM}$ \\
& imidazole & imidazole & imidazole & EDTA
\end{tabular}




\subsubsection{Enzyme concentration determination}

Enzyme concentrations were determined using the Protein $\mathrm{A}_{280}$ setting on a NanoDrop ND-1000 spectrophotometer (Thermo Fisher Scientific; Waltham, $M A, U S A)$, according to the manufacturer's instructions. The protein concentration (using the reference 1 Absorbance unit $=1 \mathrm{mg} \cdot \mathrm{mL}^{-1}$ ) was then divided by its specific calculated $1 \mathrm{mg} \cdot \mathrm{mL}^{-1}$ Absorbance unit (calculation performed by entering the protein amino acid sequence into the online software ProtParam http://web.expasy.org/protparam/ using the EXPASY server (the proteomic server of Swiss Institute of Bioinformatics)).

\subsubsection{Enzyme storage}

Purified proteins (mixed 1:1 with 80\% glycerol) were aliquoted into microcentrifuge tubes and stored at $-80^{\circ} \mathrm{C}$ for up to six months. Once defrosted, they were stored at $-20^{\circ} \mathrm{C}$ for up to one week, and disposed of after this point to avoid any loss of protein activity.

\subsection{In vitro purified protein kinetics}

\subsubsection{Determination of S33 and NADPH extinction coefficients}

Both the oxidation of $\mathrm{NAD}(\mathrm{P}) \mathrm{H}$ and the reduction of $\mathrm{S} 33$ substrates (absorbance maximas typically near $320 \mathrm{~nm}$; Olekhnovich et al., (2009); contribute to the decrease in light absorbance at wavelengths ranging from 320 to $360 \mathrm{~nm}$ as the oxidoreductase reaction proceeds; Varghese and Whitmore, 1980). As such, combined molar extinction coefficients encompassing the change in absorbance due to both compounds were derived as previously described (Olekhnovich et al., 2009).

The molar extinction coefficient for reduction of S33 combined the $350 \mathrm{~nm}$ extinction coefficient of the S33 (experimentally determined to be $1,610 \mathrm{M}^{-1}$ $\left.\mathrm{cm}^{-1}\right)$ with the assumed oxidation of two moles of $\operatorname{NAD}(\mathrm{P}) \mathrm{H}\left(2 \mathrm{x} \varepsilon=4,020 \mathrm{M}^{-1}\right.$ $\mathrm{cm}^{-1}$ ) for every mole of S33 reduced, with a final extinction of $9,650 \mathrm{M}^{-1} \mathrm{~cm}^{-1}$ used. The extinction coefficient for S33 was experimentally determined by 
measuring the decrease in $350 \mathrm{~nm}$ absorbance (-0.161) that occurred when $100 \mu \mathrm{M}$ of S33 was incubated with $300 \mu \mathrm{M}$ NADPH and excess NfsA_Ec (purified enzyme) for $30 \mathrm{~min}$. NfsA_Ec was used as it possesses low-level NADPH oxidase activity, such that it slowly reduces dissolved oxygen in the buffer, ensuring the eventual oxidation of all NADPH remaining in the cuvette post full reduction of S33. The extinction coefficient of NADPH (0.402 ) was experimentally determined by measuring the decrease in $350 \mathrm{~nm}$ absorbance when $100 \mu \mathrm{M}$ NADPH was incubated with excess NfsA_Ec (purified enzyme) for $30 \mathrm{~min}$.

\subsubsection{Glucose oxidase-catalase oxygen scavenging system}

A glucose oxidase-catalase oxygen scavenging system was employed for S33 enzyme kinetics to reduce the amount of oxygen in the reaction and subsequently the level of background nitroreductase NAD(P)H oxidase activity taking place. The system consisted of 12 units $\mathrm{mL}^{-1}$ glucose oxidase from Aspergillus niger (Sigma-Aldrich; St. Louis, MO, USA), 12 units $\mathrm{mL}^{-1}$ catalase from bovine liver (Sigma-Aldrich; St. Louis, MO, USA), and $50 \mathrm{mM}$ glucose added to the Uvette ${ }^{\mathrm{TM}}$ already containing Tris- $\mathrm{Cl} \mathrm{pH} 7.0$ and DMSO and/or S33 (Section 2.13.3) and the reaction left to incubate at room temperature for 6 minutes prior to addition of enzyme and NADPH and commencement of kinetic reaction.

\subsubsection{Purified protein kinetics}

Apparent steady state enzyme kinetics for nitroreductases and S33 were assessed by the monitoring of decreases in absorbance at $350 \mathrm{~nm}$, with extinction coefficient determined as outlined in Section 2.13.1. Reactions were carried out in $60 \mu \mathrm{L}$ volumes in UVettes ${ }^{\mathrm{TM}}$ (Eppendorf, Hamburg, Germany), using a $2 \mathrm{~mm}$ path length, and the wavelength was measured using a Helios y UV-Vis spectrophotometer (Thermo Fisher Scientific; Waltham, MA, USA). Reactions were conducted in $10 \mathrm{mM}$ Tris-Cl pH 7.0 
buffer and consisted of 4\% DMSO and S33 concentrations ranging from 0$1200 \mu \mathrm{M}$. Where relevant, a glucose oxidase-catalase oxygen scavenging system comprising 12 units $\mathrm{mL}^{-1}$ glucose oxidase from Aspergillus niger (Sigma-Aldrich; St. Louis, MO, USA), 12 units $\mathrm{mL}^{-1}$ catalase from bovine liver (Sigma-Aldrich; St. Louis, MO, USA), and $50 \mathrm{mM}$ glucose (Section 2.13.2) was included in the Uvette ${ }^{\mathrm{TM}}$ and left for 6 minutes prior to commencement of the nitroreductase reaction. Reactions were initiated by addition of 250 $\mu \mathrm{M}$ NADPH and $6 \mu \mathrm{L}$ of an appropriate dilution of enzyme and the linear decrease in absorbance monitored for the first $60 \mathrm{~s}$ post enzyme addition. Where possible, reaction rates were measured at substrate concentrations ranging from $0.2-5 \mathrm{x}$ apparent $K_{M}$, although for some enzymes this was not possible as the sensitivity and linear absorbance maxima of the spectrophotometer meant only concentrations ranging from 20-1200 $\mu \mathrm{M}$ substrate could be accurately assayed. Non-linear regression analyses and Michaelis-Menten curve fitting were performed using GraphPad Prism 7.0 (GraphPad Software Inc.; La Jolla, CA, USA).

\subsection{Sodium Dodecyl Sulphate Polyacrylamide Gel Electrophoresis (SDS-}

\section{PAGE)}

\subsubsection{SDS-PAGE}

For visualisation of proteins, SDS-PAGE gels were cast using a BioRad MiniPROTEAN $®$ Tetra Cell Casting Stand. Roughly $5 \mathrm{~mL}$ of separating gel (Table 2-14) was applied to the gel cast, covered with $1 \mathrm{~mL}$ of $100 \%$ drum isopropanol and left to set for $30 \mathrm{~min}$. The isopropanol was then discarded and roughly $3 \mathrm{~mL}$ of $5 \%$ stacking gel poured over, a multi-well comb inserted and the gel left to solidify for $30 \mathrm{~min} .10 \mu \mathrm{L}$ of PageRuler ${ }^{\mathrm{TM}}$ Plus Prestained Protein Ladder (10 to $250 \mathrm{kDa}$; Thermo Fisher Scientific; Waltham, MA, USA) and treated protein samples were loaded into the wells and gels were run in 1x SDS Run buffer at constant voltage $(150 \mathrm{~V})$ for approximately $60 \mathrm{~min}$, roughly corresponding to the time at which the dye front ran off the gel.

Protein bands were stained by gentle shaking in Coomassie blue stain 
solution for $30 \mathrm{~min}$. Destaining was carried out by washing the gel of all Coomassie blue stain in running tap water, followed by gentle shaking in Destain solution for as long as necessary to achieve the desired contrast. Protein size was determined by comparison to the Protein ladder.

\subsubsection{SDS-PAGE of His-tag purified proteins for enzyme kinetics}

Cells containing pET28a+:nitroreductase were expressed using the protocol in Section 2.12.1. $20 \mu \mathrm{L}$ aliquots of samples taken for SDS-PAGE analysis included: a pre-induction sample, an induced sample, soluble and insoluble fraction samples, flow-through from the Nickel resin column for each bind and wash step, the pooled eluted protein, and protein following desalting. These were mixed 1:2 with 3x SDS-PAGE loading buffer and heated at $95^{\circ} \mathrm{C}$ for 10 minutes before $10 \mu \mathrm{L}$ run out on a $15 \%$ separating SDS-PAGE gel.

\subsubsection{SDS-PAGE of intracellular over-expressed nitroreductases}

Cells containing pUCX:nitroreductase or pET:nitroreductase were inoculated with $200 \mu \mathrm{L} \mathrm{LB}+\mathrm{Amp}+0.2 \%$ (w/v) glucose overnight at $30{ }^{\circ} \mathrm{C}$ in a 96-well plate, $200 \mathrm{rpm}$. The following day $100 \mu \mathrm{L}$ of overnight was used to inoculate $2 \mathrm{~mL} \mathrm{LB}+\mathrm{Amp}+0.2 \%(\mathrm{w} / \mathrm{v})$ glucose + IPTG (50 mM unless stated otherwise) in $15 \mathrm{~mL}$ centrifuge tubes, $30^{\circ} \mathrm{C}$ for $4 \mathrm{~h}$. The cell pellet was harvested and resuspended in an appropriate volume of media such that a 1:500 dilution would have an $\mathrm{OD}_{600}$ of 0.100 , and $20 \mu \mathrm{L}$ of the undiluted sample was mixed 1:3 with 3x SDS-PAGE loading buffer. This was heated at $95^{\circ} \mathrm{C}$ for 10 minutes before $10 \mu \mathrm{L}$ run out on a $12.5 \%$ separating SDS-PAGE gel. 


\subsubsection{SDS-PAGE recipes}

Table 2-14 SDS-PAGE gel recipes

\begin{tabular}{llll} 
Reagents & $\begin{array}{l}\mathbf{1 2 . 5 \%} \\
\text { separating gel }\end{array}$ & $\begin{array}{l}\mathbf{1 5 \%} \text { separating } \\
\text { gel }\end{array}$ & $\begin{array}{l}\mathbf{5 \%} \text { stacking } \\
\text { gel }\end{array}$ \\
\hline 2\% Bisacrylamide & $0.81 \mathrm{~mL}$ & $1.0 \mathrm{~mL}$ & $0.16 \mathrm{~mL}$ \\
Linear acrylamide & $1.52 \mathrm{~mL}$ & $1.82 \mathrm{~mL}$ & $0.29 \mathrm{~mL}$ \\
$1.5 \mathrm{M}$ Tris-HCl pH 8.8 & $1.13 \mathrm{~mL}$ & $1.13 \mathrm{~mL}$ & - \\
\hline $0.5 \mathrm{M}$ Tris-HCl pH 6.8 & - & - & $0.75 \mathrm{~mL}$ \\
$10 \%(w / v)$ SDS & $50 \mu \mathrm{L}$ & $50 \mu \mathrm{L}$ & $30 \mu \mathrm{L}$ \\
$10 \%$ APS $^{\text {a }}$ & $38 \mu \mathrm{L}$ & $38 \mu \mathrm{L}$ & $23 \mu \mathrm{L}$ \\
TEMED $^{\text {a }}$ & $3.75 \mu \mathrm{L}$ & $3.75 \mu \mathrm{L}$ & $3.75 \mu \mathrm{L}$ \\
\hline diH $_{2} \mathrm{O}$ & $1.43 \mathrm{~mL}$ & $0.95 \mathrm{~mL}$ & $1.74 \mathrm{~mL}$ \\
TOTAL $^{\text {TOT }}$ & $5 \mathrm{~mL}$ & $5 \mathrm{~mL}$ & $3 \mathrm{~mL}$ \\
\hline
\end{tabular}

${ }^{a}$ Added just prior to pouring of gel

Table 2-15 SDS buffer recipes

\begin{tabular}{|c|c|c|c|}
\hline $\begin{array}{l}\text { 3x SDS-PAGE } \\
\text { loading buffer }\end{array}$ & SDS run buffer & $\begin{array}{l}\text { Coomassie stain } \\
\text { solution }\end{array}$ & $\begin{array}{l}\text { Destain } \\
\text { solution }\end{array}$ \\
\hline $\begin{array}{l}187.5 \text { mM Tris- } \\
\mathrm{HCl} \mathrm{pH} 6.8\end{array}$ & $\begin{array}{l}25 \text { mM Tris- } \\
\mathrm{HCl} \mathrm{pH} 8.3\end{array}$ & $\begin{array}{l}0.1 \%(\mathrm{w} / \mathrm{v}) \\
\text { Coomassie brilliant } \\
\text { blue R250 }\end{array}$ & $\begin{array}{l}30 \% \\
\text { ethanol }\end{array}$ \\
\hline $6 \%(\mathrm{w} / \mathrm{v}) \mathrm{SDS}$ & $\begin{array}{l}192 \mathrm{mM} \\
\text { glycine }\end{array}$ & $41.6 \%$ methanol & $\begin{array}{l}10 \% \text { acetic } \\
\text { acid }\end{array}$ \\
\hline $\begin{array}{l}15 \%(\mathrm{w} / \mathrm{v}) \beta- \\
\text { mercaptoethanol } \\
30 \%(\mathrm{v} / \mathrm{v}) \text { glycerol } \\
0.006 \%(\mathrm{w} / \mathrm{v}) \\
\text { bromophenol } \\
\text { blue }\end{array}$ & $0.1 \%(\mathrm{w} / \mathrm{v}) \mathrm{SDS}$ & $0.168 \%$ acetic acid & \\
\hline
\end{tabular}

\subsection{Assays for nitroreductase activity with nitroaromatic substrates}

\subsubsection{SOS assay}

\subsubsection{SOS assay using a lacZ reporter in SOS-R2}

Individual microtitre plate wells containing $200 \mu \mathrm{L} L B+A m p+0.4 \%(w / v)$ glucose were inoculated with SOS-R2 pUCX:nitroreductase strains and incubated overnight at $30^{\circ} \mathrm{C}, 200 \mathrm{rpm} .15 \mu \mathrm{L}$ of the overnight cultures were used to inoculate $200 \mu \mathrm{L}$ of $\mathrm{LB}+\mathrm{Amp}+50 \mu \mathrm{M}$ IPTG $+0.2 \%$ glucose in a sterile microtitre plate and incubated at $30{ }^{\circ} \mathrm{C}, 200 \mathrm{rpm}$ for $3 \mathrm{~h}$. Cultures 
were then split into $100 \mu \mathrm{L}$ duplicates into a clean microtitre plate and supplemented with either $100 \mu \mathrm{L}$ challenge media (assay media +prodrug, $2 \%$ DMSO) or $100 \mu \mathrm{L}$ control media (assay media $+2 \%$ DMSO). OD $_{600}$ readings were recorded using an EnSpire ${ }^{\mathrm{TM}} 2300$ Multilabel Reader (PerkinElmer, Waltham, MA, USA) and the plate returned to the incubator at $30{ }^{\circ} \mathrm{C}, 200 \mathrm{rpm}$. Following 4 hours the plates were removed, $\mathrm{OD}_{600}$ readings recorded and $10 \mu \mathrm{L}$ cells removed for $\beta$-galactosidase analysis. The $10 \mu \mathrm{L}$ aliquots were added to $90 \mu \mathrm{L}$ of $40 \mathrm{mM}$ sodium phosphate buffer $\mathrm{pH} 7.0$ and $50 \mu \mathrm{L}$ ZOB buffer (4 parts Z buffer, 1 part T-Base, see Table 2-16 for recipes; Alksne et al., (2000)) in a sterile microtitre plate, which was incubated at 37 ${ }^{\circ} \mathrm{C}$ without shaking for 10 - 15 minutes until visible colour development from the ortho-Nitrophenyl- $\beta$-galactoside (ONPG) substrate was observed. Reactions were then terminated by addition of $50 \mu \mathrm{L}$ of $1 \mathrm{M} \mathrm{Na}_{2} \mathrm{CO}_{3}$ to each well. Absorbance readings at $420 \mathrm{~nm}$ and $550 \mathrm{~nm}$ were recorded and Miller units were calculated by the Miller equation $\left(1\right.$ Miller Unit $=\left(\mathrm{OD}_{420}-[1.75 \times\right.$ $\left.\left.\mathrm{OD}_{550}\right]\right) /\left(\mathrm{OD}_{600} \times \mathrm{t} \times \mathrm{v}\right)$; Miller, 1972). The SOS response was graphed as challenged Miller units - unchallenged Miller units.

Table 2-16 Recipes of buffers required for measurement of $\beta$-galactosidase activity in LacZ SOS assays

\begin{tabular}{|c|c|c|}
\hline $\begin{array}{l}1 \text { M sodium phosphate } \\
\text { buffer } \mathrm{pH} 7.0\end{array}$ & Z-buffer & T-base \\
\hline $\mathrm{Na}_{2} \mathrm{HPO}_{4} 577 \mathrm{mM}$ & $\mathrm{Na}_{2} \mathrm{HPO}_{4} 126 \mathrm{mM}$ & $\mathrm{K}_{2} \mathrm{HPO}_{4} 80 \mathrm{mM}$ \\
\hline \multirow[t]{6}{*}{$\mathrm{NaH}_{2} \mathrm{PO}_{4} 423 \mathrm{mM}$} & $\mathrm{NaH}_{2} \mathrm{PO}_{4} 74 \mathrm{mM}$ & $\mathrm{KH}_{2} \mathrm{PO}_{4} 44 \mathrm{mM}$ \\
\hline & $\mathrm{MgSO}_{4} 2 \mathrm{mM}$ & $\left(\mathrm{NH}_{4}\right)_{2} \mathrm{SO}_{4} 15.1 \mathrm{mM}$ \\
\hline & $\mathrm{MnSO}_{4} 400 \mu \mathrm{M}$ & $\begin{array}{l}\text { Tris-sodium citrate } \\
\text { 1g. } \mathrm{L}^{-1}\end{array}$ \\
\hline & CTAB 399 mg.L $\mathrm{L}^{-1}$ & \multirow[t]{3}{*}{$\mathrm{ONPG}^{\mathrm{b}} 8 \mathrm{mg} \cdot \mathrm{mL}^{-1}$} \\
\hline & $\begin{array}{l}\text { Sodium deoxycholate } \\
199.5 \mathrm{mg} . \mathrm{L}^{-1}\end{array}$ & \\
\hline & $\begin{array}{l}\beta \text {-mercaptoethanol } \\
174 \mathrm{mM}\end{array}$ & \\
\hline
\end{tabular}

${ }^{a} \beta$-mercaptoethanol was added to Z-buffer directly prior to use. Z-buffer without $\beta$-mercaptoethanol was stored long-term at $4{ }^{\circ} \mathrm{C}$.

${ }^{b}$ After ONPG was added, T-base was stored in aliquots at $-20{ }^{\circ} \mathrm{C}$. 


\subsubsection{SOS assay using a GFP reporter in SOS-R4}

Individual microtitre plate wells containing $200 \mu \mathrm{L} L B+\mathrm{Amp}+\mathrm{Spec}+0.2 \%$ $(\mathrm{w} / \mathrm{v})$ glucose were inoculated with SOS-R4 pUCX:nitroreductase strains and incubated overnight at $30^{\circ} \mathrm{C}, 200 \mathrm{rpm} .15 \mu \mathrm{L}$ of the overnight cultures were used to inoculate $200 \mu \mathrm{L}$ of fresh assay media $(\mathrm{LB}+\mathrm{Amp}+\mathrm{Spec}+50 \mu \mathrm{M}$ IPTG $+0.2 \%(\mathrm{w} / \mathrm{v})$ glucose $)$ in a sterile microtitre plate and incubated at 30 ${ }^{\circ} \mathrm{C}, 200 \mathrm{rpm}$ for $2-2.5 \mathrm{~h}$. Cultures were then split into four $30 \mu \mathrm{L}$ aliquots in a clean 384-well microtitre plate, two wells supplemented with $30 \mu \mathrm{L}$ challenge media (assay media + drug, 2\% DMSO), and two wells supplemented with $30 \mu \mathrm{L}$ control media (assay media $+2 \%$ DMSO), and the plate returned to the incubator. Following 4 hours of incubation plates were removed, and $\mathrm{OD}_{600}$ and fluorescence (excitation $490 \mathrm{~nm} /$ emission $503 \mathrm{~nm}$ ) readings recorded using an EnSpire ${ }^{\mathrm{TM}} 2300$ Multilabel Reader (PerkinElmer, Waltham, MA, USA). Relative fluorescence for each well was calculated by dividing the fluorescence reading by $\mathrm{OD}_{600}$, and fold increase in fluorescence calculated by dividing the relative fluorescence for a challenged well by its unchallenged counterpart.

\subsubsection{Fluorescence-activated cell sorting (FACS) of nitroreductase libraries}

Variant libraries in SOS-R4 selected with $0.5 \mu \mathrm{M}$ NCS as per Section 2.11.1 were used to inoculate 10 wells of a 96 well microplate, each filled with 200 $\mu \mathrm{L}$ of $\mathrm{LB}+\mathrm{Amp}+\mathrm{Spec}+0.4 \%(\mathrm{w} / \mathrm{v})$ glucose, along with two wells each of a wild-type nitroreductase and empty pUCX control, and grown overnight at $30{ }^{\circ} \mathrm{C}, 200 \mathrm{rpm}$. The next morning cultures from these wells were pooled and $10 \times 15 \mu \mathrm{L}$ aliquots used to inoculate $200 \mu \mathrm{L}$ apiece of M9 assay media (M9 minimal media + Amp + Spec $+50 \mu \mathrm{M}$ IPTG) in a sterile microtitre plate. Cultures were grown for $2 \mathrm{~h}$, split into $100 \mu \mathrm{L}$ duplicates and challenged with varying concentrations of S33 $(0.5-10 \mu \mathrm{M})$ as described in the standard GFP-based SOS assay, except with M9 minimal media used in place of LB (Section 2.15.1.2). After 4 hours incubation, $10 \mu \mathrm{L}$ of the unchallenged and drug-challenged culture were diluted into $1000 \mu \mathrm{L}$ M9 in FACS tubes. 
Samples were analysed on a FACS Vantage SE-Diva (Becton Dickinson Biosciences, Bedford, MA) with GFP fluorescence excited using a $100 \mathrm{~mW}$ blue laser $(488 \mathrm{~nm})$ and detected using a photomultiplier tube with a 530/30 nm band pass filter. Gates for sorting were set using the challenged wild-type nitroreductase (positive fluorescence) and unchallenged wild-type nitroreductase (negative control). Sorted events were collected, spun down in a microcentrifuge tube and then resuspended in $1 \mathrm{~mL}$ of $\mathrm{LB}+\mathrm{Amp}+\mathrm{Spec}$ $+0.4 \%(\mathrm{w} / \mathrm{v})$ glucose and allowed to recover at $37{ }^{\circ} \mathrm{C} 200 \mathrm{rpm}$ for 1 hour before plating in $100 \mu \mathrm{L}$ aliquots on solid media plates containing Spec + Amp antibiotics. Plates were grown overnight at $37^{\circ} \mathrm{C}$, before colonies were picked individually into separate media filled wells of sterile microtitre plates. These plates were grown overnight, mixed with glycerol and then stored at $-80{ }^{\circ} \mathrm{C}$ until required to inoculate day cultures for activity screening with nitroaromatic substrates.

\subsubsection{Growth inhibition assay}

Individual microtitre plate wells containing $200 \mu \mathrm{L} L B+A m p+0.2 \%(w / v)$ glucose were inoculated with SOS-R2 or 7NT pUCX:nitroreductase strains and incubated overnight at $30{ }^{\circ} \mathrm{C}, 200 \mathrm{rpm} .15 \mu \mathrm{L}$ of the overnight cultures were used to inoculate $200 \mu \mathrm{L}$ of fresh assay media $(\mathrm{LB}+\mathrm{Amp}+50 \mu \mathrm{M}$ IPTG $+0.2 \%(\mathrm{w} / \mathrm{v})$ glucose) in a sterile microtitre plate and incubated at $30{ }^{\circ} \mathrm{C}, 200$ rpm for $2-2.5 \mathrm{~h}$. Cultures were then split into four $30 \mu \mathrm{L}$ aliquots in a sterile 384-well microtitre plate, two wells supplemented with $30 \mu \mathrm{L}$ challenge media (assay media + drug, 2\% DMSO), and two wells supplemented with 30 $\mu \mathrm{L}$ control media (assay media $+2 \% \mathrm{DMSO}$ ). $\mathrm{OD}_{600}$ readings were recorded using an EnSpire ${ }^{\mathrm{TM}} 2300$ Multilabel Reader (PerkinElmer, Waltham, MA, USA) and the plate returned to the incubator. Following 4 hours, plates were removed, and $\mathrm{OD}_{600}$ readings recorded. Relative increases in $\mathrm{OD}_{600}$ for challenged vs unchallenged wells were then used to calculate percentage growth inhibition. 


\subsubsection{IC 50 assay}

Individual microtitre plate wells containing $200 \mu \mathrm{L} \mathrm{LB}+\mathrm{Amp}+0.2 \%(\mathrm{w} / \mathrm{v})$ glucose were inoculated with SOS-R2 or 7NT pUCX:nitroreductase strains and incubated overnight at $30{ }^{\circ} \mathrm{C}, 200 \mathrm{rpm} .100 \mu \mathrm{L}$ of the overnight cultures were used to inoculate $2 \mathrm{~mL}$ of LB + Amp + IPTG (concentrations ranged from $5 \mu \mathrm{M}$ up to $250 \mu \mathrm{M})+0.2 \%$ (w/v) glucose in sterile $15 \mathrm{~mL}$ centrifuge tubes and incubated at $30{ }^{\circ} \mathrm{C}, 200 \mathrm{rpm}$ for $2 \mathrm{~h} .40 \mu \mathrm{L}$ aliquots of each culture were added to wells of a sterile 384 well microplate containing $40 \mu \mathrm{L}$ of assay media $\pm 2 \mathrm{x}$ final drug concentration. Each culture was exposed, in duplicate, to at least seven wells containing a two-fold increasing titration of drug and one media only control. Where drug solubility in the assay media allowed, the highest concentration selected for each drug in these assays allowed for over $50 \%$ growth inhibition of strains bearing nitroreductases of interest. Media was supplemented with DMSO as appropriate and the DMSO in media never exceeded 4\%. Percentage growth inhibition at each drug concentration was determined as per the growth inhibition assay (Section

2.15.3) and $\mathrm{IC}_{50}$ values (the drug concentration which caused $50 \%$ strain growth inhibition) were calculated using dose-response inhibition response and a four-parameter variable slope in GraphPad Prism 7.0 (GraphPad Software Inc.; La Jolla, CA, USA).

\subsubsection{Biological and technical replicates}

For experiments described in this thesis, biological replicates (i.e. those that evaluate variation within the system under investigation) refer to independent overnight and day cultures of a particular nitroreductase overexpressing E. coli strain. Technical replicates (i.e. those that evaluate variation due to measurement) refer to assays performed using the same overnight and day cultures but in different wells. 


\subsection{Creation of metagenomic libraries}

\subsubsection{Preparation of genomic DNA}

Genomic DNA from Gram-positive and Gram-negative bacterial species was extracted using the genomic DNA preparation protocol in the book Practical Streptomyces Genetics (Kieser, 2000). In short, bacterial species were grown overnight in $100 \mathrm{~mL} \mathrm{LB}$ at $37^{\circ} \mathrm{C}, 200 \mathrm{rpm}$. The following day, cultures were pelleted at $4000 \mathrm{~g}$ for $10 \mathrm{~min}$, the supernatant discarded and the pellet resuspended in $1.5 \mathrm{~mL}$ SET buffer $(75 \mathrm{mM} \mathrm{NaCl}, 25 \mathrm{mM}$ EDTA pH 8.0, $20 \mathrm{mM}$ Tris- $\mathrm{HCl} \mathrm{pH} 7.5$ ) also containing $1.25 \mathrm{mg}$ lysozyme and incubated at $37^{\circ} \mathrm{C}$ for $1 \mathrm{~h} .0 .7 \mathrm{mg}$ proteinase $\mathrm{K}$ and $150 \mu \mathrm{L} 10 \%$ SDS was added and the solutions mixed by inversion, then incubated at $55^{\circ} \mathrm{C}$ for 2 hours with occasional inversion. $500 \mu \mathrm{L}$ of $5 \mathrm{M} \mathrm{NaCl}$ was added and inverted thoroughly to mix. $1.25 \mathrm{~mL}$ of chloroform was added and the solution inverted to mix for $30 \mathrm{~min}$. The solution was then centrifuged at max speed in $1.5 \mathrm{~mL}$ microcentrifuge tubes for 10 minutes and the top aqueous layer transferred into a fresh microcentrifuge tube. $0.6 \mathrm{x}$ volume isopropanol and $250 \mu \mathrm{g}$ RNAse A was added to each tube and the tube inverted to mix, then centrifuged at max speed for 15 minutes at $4{ }^{\circ} \mathrm{C}$. The supernatant was discarded and the pellet washed with $1 \mathrm{~mL}$ of ice cold $70 \%$ ethanol, then centrifuged for a further 15 minutes at max speed, $4{ }^{\circ} \mathrm{C}$. The supernatant was then discarded and the pellet resuspended in $100 \mu \mathrm{L}$ TE $(10 \mathrm{mM}$ Tris- $\mathrm{HCl} \mathrm{pH}$ 8.0, $0.1 \mathrm{mM}$ EDTA) and left at $55^{\circ} \mathrm{C}$ for 1 hours for the pellet to fully redissolve in the solution.

\subsubsection{Electro-elution of restriction enzyme-cleaved genomic DNA}

Restriction enzyme-cleaved genomic DNA was mixed 6:1 with 6x DNA loading dye (30\% (v/v) glycerol, $0.25 \%(\mathrm{w} / \mathrm{v})$ bromophenol blue) and run on an agarose gel (1\% w/v agarose in 1x TAE buffer ( $40 \mathrm{mM}$ Tris-acetate, $1 \mathrm{mM}$ EDTA, pH 8.3), containing 1x SYBR Safe DNA gel stain (Thermo Fisher Scientific; Waltham, MA, USA)) and covered with $1 \mathrm{x}$ TAE in a suitable horizontal electrophoresis tank at $100 \mathrm{~V}$ for $90 \mathrm{~min}$. DNA was visualised 
using a blue light transilluminator and size compared by comparison to Hyperladder I (Bioline, London, UK). The section of gel containing $1 \mathrm{~kb}-2.5 \mathrm{~kb}$ DNA fragments was excised and the DNA electro-eluted from the gel using an Electro-Eluter/Concentrator (C.B.S. Scientific Company Inc.,San Diego, CA, USA) overnight at $15 \mathrm{~mA}$. The electro-eluted DNA was concentrated using an Amicon Ultra-0.5 Centrifugal Filter Unit with Ultracel-3 membrane (Merck Millipore, Billerica, Massachusetts, USA) and washed once with TE (10 mM Tris- $\mathrm{HCl} \mathrm{pH}$ 8.0, 0.1 mM EDTA). The DNA was concentrated down to less than $200 \mu \mathrm{L}$ and concentration determined using a NanoDrop (Section 2.6.4).

\subsubsection{Top agarose selection of metagenomic libraries}

7TL cells transformed with the pET28a + multi-genomic library were plated on LB agar plates containing Kan and $50 \mu \mathrm{M}$ IPTG and incubated overnight at $37^{\circ} \mathrm{C}$. The following day a suitable volume of top agarose $(0.6 \%$ agarose (w/v), $5 \mathrm{mg} \cdot \mathrm{mL}^{-1} \mathrm{NaCl}$ ) heated to $55^{\circ} \mathrm{C}$ and containing either $5 \mathrm{mM} 4(5)$ nitroimidazole or $10 \mathrm{mM}$ 2-methyl-4(5)-nitroimidazole was poured over the agar plate. The plate was left at room temperature for up to 24 hours and changes in colour of colonies were observed at multiple time points over this period. 


\section{Chapter 3. Screening of native and targeted- mutagenesis libraries for nitroreductases capable of metabolising S33}

\subsection{Introduction}

Prior attempts by the Ackerley research group to repurpose hypoxiaactivated 2-nitroimidazole PET capable probes, such as EF5, for nitroreductase activation have been confounded by relatively high background activation under hypoxia alone (Williams, 2013). Subsequently, to enable more specific imaging of bacterial nitroreductases, Drs Smaill and Patterson at the ACSRC designed a 5-nitroimidazole PET-capable analogue of EF5, S33 (chemical structure shown in Figure 3-1), which has a lower capability to be activated by endogenous human one-electron reductases. This chapter describes the interrogation of nitroreductase candidates from multiple phylogenetically diverse bacteria species and oxidoreductase families for their ability to activate S33. The first part of this chapter describes the evaluation of wild-type nitroreductase candidates for their ability to metabolise S33, followed by the screening of nitroreductase variant libraries that had been previously created in the Ackerley lab to improve metabolism of the hypoxia-activated prodrug PR-104A, for selection of mutants with improved activation of S33.

\subsubsection{S33}<smiles>Cc1ncc([N+](=O)[O-])n1CC(=O)NCC(F)(F)F</smiles>

Figure 3-1 Chemical structures of the 5-nitroimidazole PET imaging-capable probe S33. 
The rationale behind the design of S33 involved the alteration of the 2nitroimidazole scaffold of EF5 to a 5-nitroimidazole in an attempt to lower the compound's one-electron reduction potential (an experimentally derived measure), which would decrease its ability to be activated by human one-electron reductases. Hypoxia-activated compounds such as EF5 are designed to have a reduction potential less negative than $-450 \mathrm{mV}$ to enable their activation by these reductases (Denny, 2000). However, this feature is undesirable for probes that are to be activated solely by nitroreductases, and the switching to 5-nitroimidazole or 4-nitroimidazole scaffolds could increase the negativity of their one-electron reduction potentials (Wardman and Clarke, 1976).

The switch from a 2-nitroimidazole to a 5-nitroimidazole scaffold had the effect of lowering the one-electron reduction potential from $-395 \mathrm{mV}$ for EF5 to -501 mV for S33 (Anderson et al., 2014). To allow for immunohistochemical detection of S33 entrapment both under hypoxia and following nitroreductase activation in mice xenograft tumours, S33 was designed to have an identical side chain to EF5, which allowed the use of EF5-selective fluorescent antibodies to detect S33 binding in immunohistochemistry experiments (Anderson et al., 2014).

Testing of S33 activation by human reductases in vivo showed trace levels of S33 retention in tissue when cells were exposed to the compound under strict anoxia but this was far lower than that seen with EF5, as detected using these antibodies (Figure 3-2; Anderson et al., 2014). In contrast, NfsA was found to be capable of activating both EF5 and S33 (Figure 3-3; Anderson et al., 2014). Moreover, and of great relevance to this study, although reduction of the 2-nitroimidazole EF5 yields a non-genotoxic product, the reduced form of S33 is mildly genotoxic and able to evoke the $E$. coli SOS response (Anderson et al., 2014; Williams, 2013), providing an additional mechanism for monitoring activity using SOS-responsive reporter strains of E. coli (e.g. Copp et al., 2017; Prosser et al., 2013). 
A
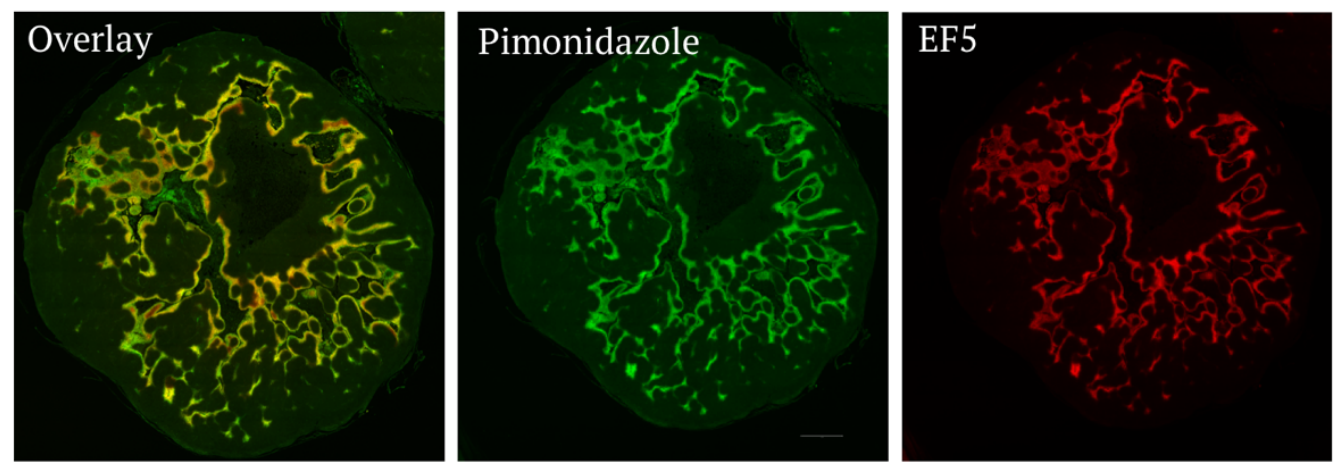

B
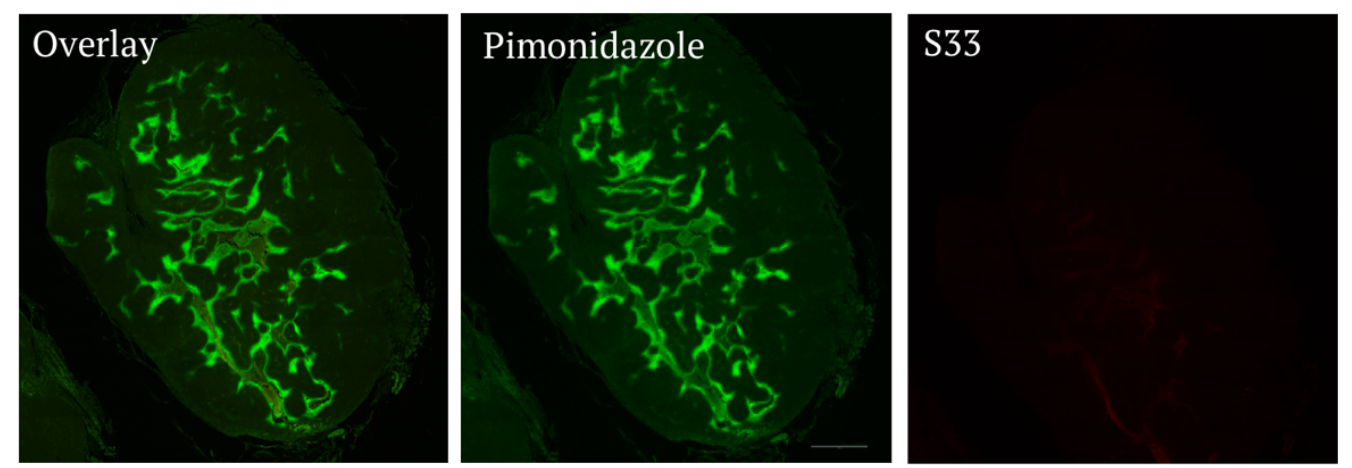

Figure 3-2 Hypoxia dependent binding of A) EF5 and B) S33 in a mouse HCT-116 tumour xenograft model. Subcutaneous HCT-116 tumours were established in NIH III nude mice and grown to $\sim 10 \mathrm{~mm}$ diameter. Mice were treated with the 2-nitroimidazole hypoxia-activated probe pimonidazole and either EF5 or S33 (dosing of all compounds at $60 \mathrm{mg} / \mathrm{kg}$ via intraperitoneal injection) and tumours excised, fixed, paraffin embedded, sectioned and mounted on glass slides for immunohistochemical staining with $(40 \mu \mathrm{g} / \mathrm{mL})$ anti-pimonidazole antibody FITC-Hypoxyprobe-1 (Chemicon ${ }^{\circledR}$ International, clone 4.3.11.3) followed by staining with $(75 \mu \mathrm{g} / \mathrm{mL})$ anti-EF5/S33 antibody Cy5-ELK5.1. Images were acquired and overlaid using a Zeiss LSM 710 confocal microscope ( $x 20$ magnification - $1 \mathrm{~mm}$ scale marked on pimonidazole images) and Zen2010 software. The separate images taken to detect pimonidazole binding (green) and S33/EF5 (red) binding are depicted both individually (middle and right-hand panels) and in overlay (left panel; dual registration appearing as yellow). Figure from Anderson et al., (2014) with permission of Dr Ackerley. 


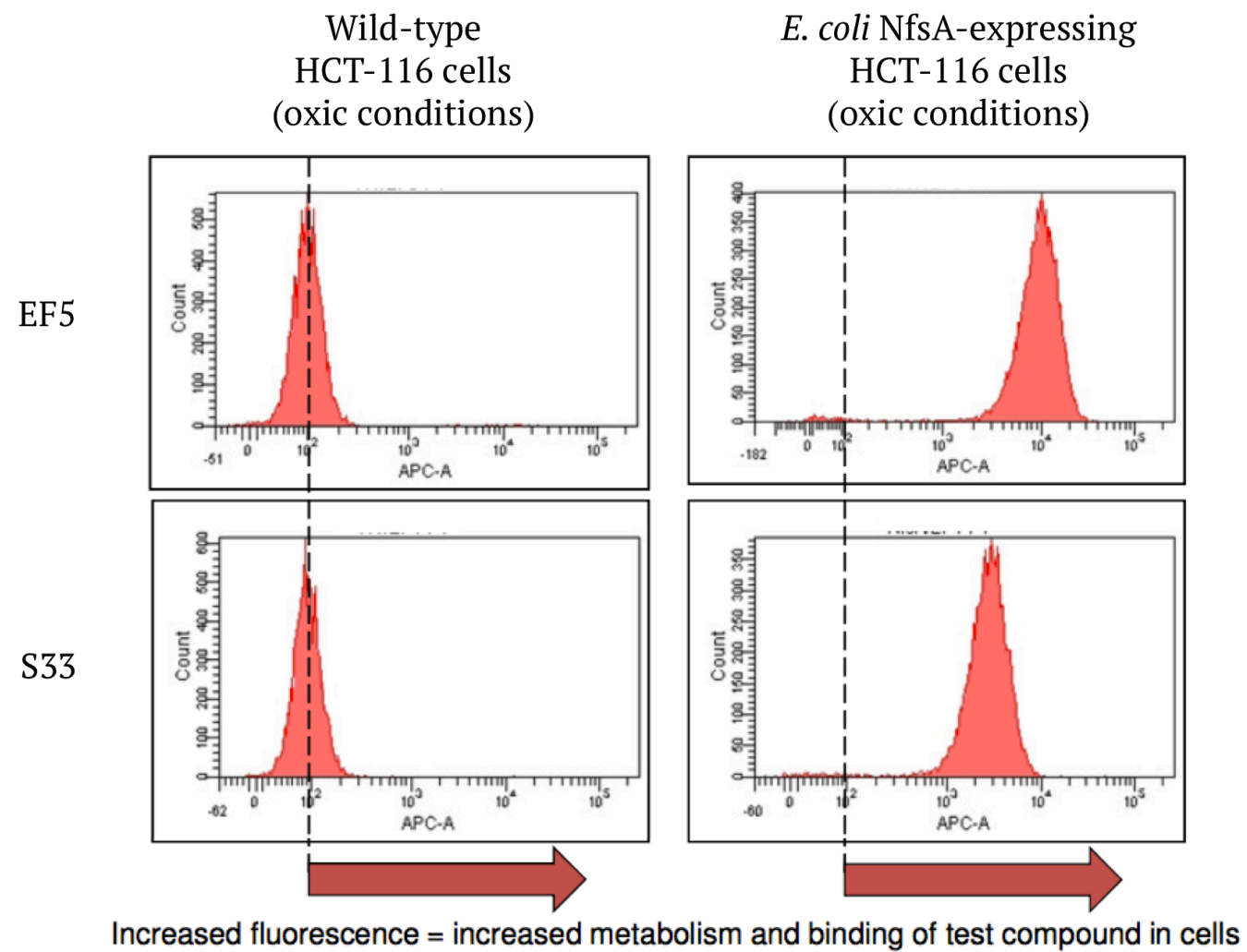

Figure 3-3 Flow cytometry analysis of wild-type HCT-116 cells or HCT-116 cells stably expressing E. coli NfsA after in vitro exposure to EF5 or S33. Wild-type HCT-116 cells (left panels) or HCT-116 cells stably expressing E. coli Nfs A (right panels) were exposed to $20 \mu M$ EF5 (top panels) or S33 (bottom panels) in vitro under oxic conditions for 2 hours. Samples were fixed and stained with EF5 antibody Alexa 488 ELK3.51 at $100 \mu \mathrm{g} / \mathrm{ml}$ and analysed on a Becton Dickinson FACscan flow cytometer. Figure reproduced from Anderson et al., (2014) with permission of Dr Ackerley.

\subsubsection{Expanded nitroreductase candidate library}

Numerous researchers in the Ackerley group have collaborated in the construction of an E. coli over-expression library that to date comprises 58 nitroreductase candidate enzymes from 20 different bacterial species. This library has been used for rapid screening of a variety of oxidoreductase enzymes for different biotechnological purposes, including activation of DNA-damaging prodrugs (Little, 2015; Prosser et al., 2013), activation of imaging probes (Williams, 2013), chromate reduction for bioremediation purposes (Robins et al., 2013), and activation of novel nitro-quenched fluorogenic probes (Horvat, 2012). The creation of a 47-membered library 
was first described by Prosser et al. (2013); this was later expanded to a panel of 58 members (Williams, 2013). In brief, orthologues of E. coli oxidoreductases NfsA, NfsB, AzoR, NemA, YieF, WrbA, YdjA, YcdI, YcaK, $\mathrm{MdaB}, \mathrm{KefF}$, and two additional reductases previously shown to metabolise CB1954 - the rat oxidoreductase NQO1 (Knox et al., 1988b) and the enzyme YwrO from B. amyloliquefaciens (Anlezark et al., 2002) were identified within genome-sequenced culturable bacterial species, amplified by PCR, and cloned into the expression vector pUCX (Figure 2-1). YdgI from Bacillus subtilis and NfsB from Pseudomonas aeruginosa were both previously described as NfsB orthologues (Prosser et al., 2013); however they have since been updated to belong to the Nitroreductase 1 family as indicated by NCBI BLAST results. The full list of enzymes and corresponding phylogenetic family tree are shown in Table 2-12 and Figure 2-8 respectively. Each oxidoreductase enzyme is referred to in this study by standard nomenclature (where names given from previous characterisation were retained, e.g. YcnD from Bacillus subtilis (Kunst et al., 1997); else the nitroreductase family to which the enzyme belonged to was assigned) followed by a two-letter abbreviation of the genus and species of bacteria from which the enzyme originated (Table $2-12$ ).

For the research described in this chapter, nitroreductases from the 58 nitroreductase core library were cloned into the $E$. coli screening strain $7 \mathrm{NT}$ (carrying knockouts of seven endogenous oxidoreductases - $n f s A, n f s B$, yieF, ycaK, $m d a B, a z o R$, nemA - and the efflux pump gene tolC; Copp et al., (2014)), and/or co-expressed with the plasmid pANODuet-1:GFP (Figure 2-5) for SOS assays, as explained in Sections 3.1.3 and 3.1.4.

\subsubsection{LacZ SOS assay}

To efficiently assess a large number of nitroreductases using low concentrations of bespoke compounds (e.g. S33), a simple, accurate and rapid microplate-based screening assay had previously been developed and optimized by the Ackerley group (Prosser et al., 2010a). Based on the SOS 
chromotest assay that was first developed to assess DNA-damaging agents by Quillardet et al., (1982), our assay allowed for the detection of nitroreductase-catalysed activation of DNA damaging prodrugs (Prosser et al., 2010a). The basis of this assay involves the endogenous E. coli SOS response, which is a group of around 40 genes (mostly involved in DNA repair) that are activated following DNA damage, resulting in cleavage of the SOS repressor LexA (Michel, 2005). Quillardet et al. discovered that the placement of a lacZ gene under control of the late-stage SOS-induced promoter taken from gene $s f i A$ yielded a linear, quantitative production of $\beta$ galactosidase in response to incubation with genotoxic agents (Quillardet et al., 1982). Our group designed an E. coli screening strain called SOS-R2 comprising a lacZ gene behind a $s f i A$ promoter, with increased sensitivity generated through the deletion of four endogenous genes encoding proven nitroreductases ( $n f_{s} A, n f_{s} B, a z o R$, nem $A$ ) and the tolC gene, which codes for an outer membrane pore protein involved in active expulsion of genotoxic compounds (Prosser et al., 2013). Deletion of tolC has been shown to improve sensitivity in the SOS assay for some, but not all, nitroreductaseactivated prodrugs (Prosser et al., 2013; Williams, 2013).

Exposure of an SOS-R2 strain over-expressing a nitroreductase to sub-lethal concentrations of a nitroaromatic compound leads to nitroreductasemediated reduction of the compound, which goes on to induce the SOS response. This leads to the production of higher levels of $\beta$-galactosidase, which can cleave the reporter substrate ortho-nitrophenyl galactoside (ONPG) to yield galactose and the yellow coloured o-nitrophenol in a quantifiable fashion. The measurement of product formation thus directly correlates to the activity of the prodrug-activating nitroreductase (Figure 3-4; Prosser et al., 2013). Previous research showed that sub-lethal concentrations of S33 (at levels far above those that would be required for PET clinical imaging) could induce the LacZ SOS response in SOS-R2 (Williams, 2013). 


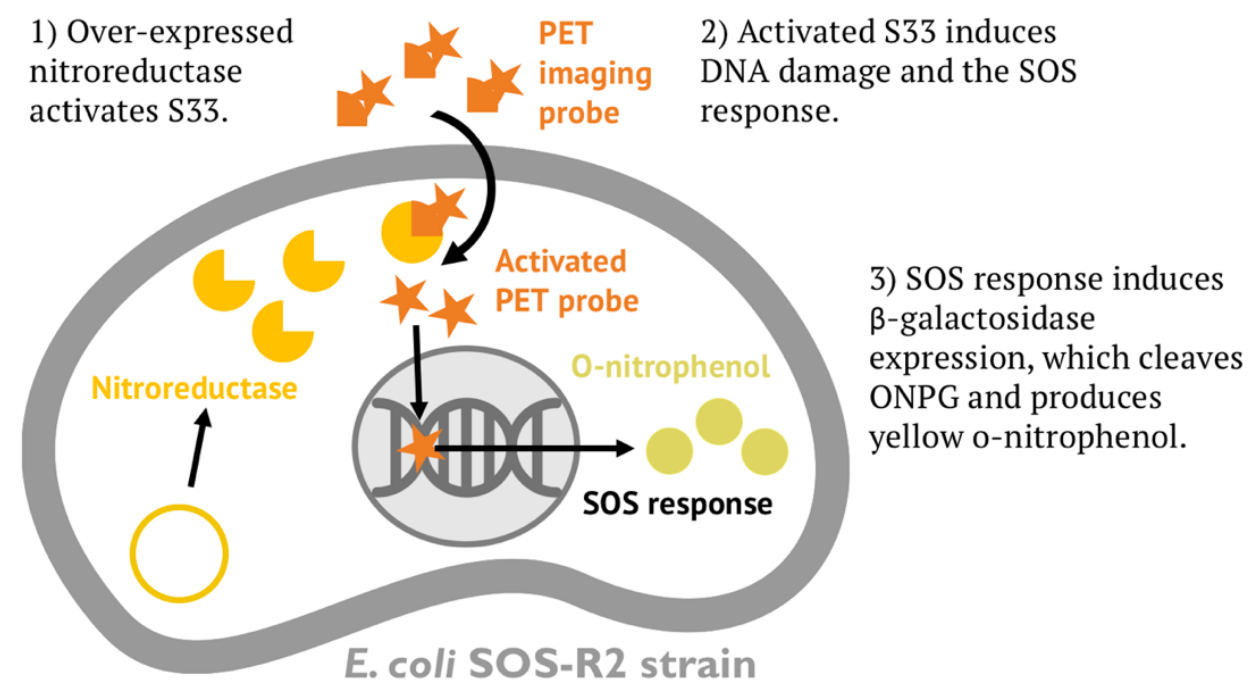

Figure 3-4 Schematic showing the LacZ SOS assay in E. coli strain SOS-R2.

\subsubsection{GFP SOS assay}

Following the design of the LacZ-based SOS screening strain, attention was turned to further improvement of this assay to a GFP-based format (Figure 3-5). This alleviated the need for cell lysis and performance of a timeconsuming activity assay, as well as allowing for increased assay sensitivity and thereby reducing the volume of prodrug required for a single 384-well microplate based assay (as opposed to the LacZ SOS assay, which is conducted in multiple 96-well plates). Furthermore, it allowed for rapid, high-throughput screening of directed evolution libraries using fluorescence-activated cell sorting (FACS) (Copp et al., 2014). This strain was generated by the introduction of the pANODuet-1:GFP plasmid (see Figure 2-5 for plasmid map) into the E. coli 7NT strain, and the strain was subsequently designated SOS-R4. The GFP plasmid was created through introduction of a fragment from pANO1, created by Norman et al. (2005), including a mutated $g f p$ gene under control of a $s f i A$ promoter and a chloramphenicol resistance cassette, into the first MCS of the plasmid pCDFDuet:1 (Copp et al., 2014). The mutated gfp gene (known in literature as gfpmut $3 b$ ) is a FACS-optimised mutated variant of Aequorea victoria $g f p$ that contains two amino acid substitutions, S65G and S72A, which enable it 
to exhibit greatly increased fluorescence intensity and also allows for excitation by a standard flow cytometry $488 \mathrm{~nm}$ argon laser (Cormack et al., 1996). A strong correlation $\left(\mathrm{R}^{2}=0.71\right)$ between the LacZ SOS response in SOS-R2 and the GFP SOS response in SOS-R4 following challenge of strains over-expressing wild-type nitroreductases with CB1954 was shown (Copp et al., 2014), suggesting that the GFP SOS assay would give an accurate prediction of activity of candidate nitroreductases with S33.

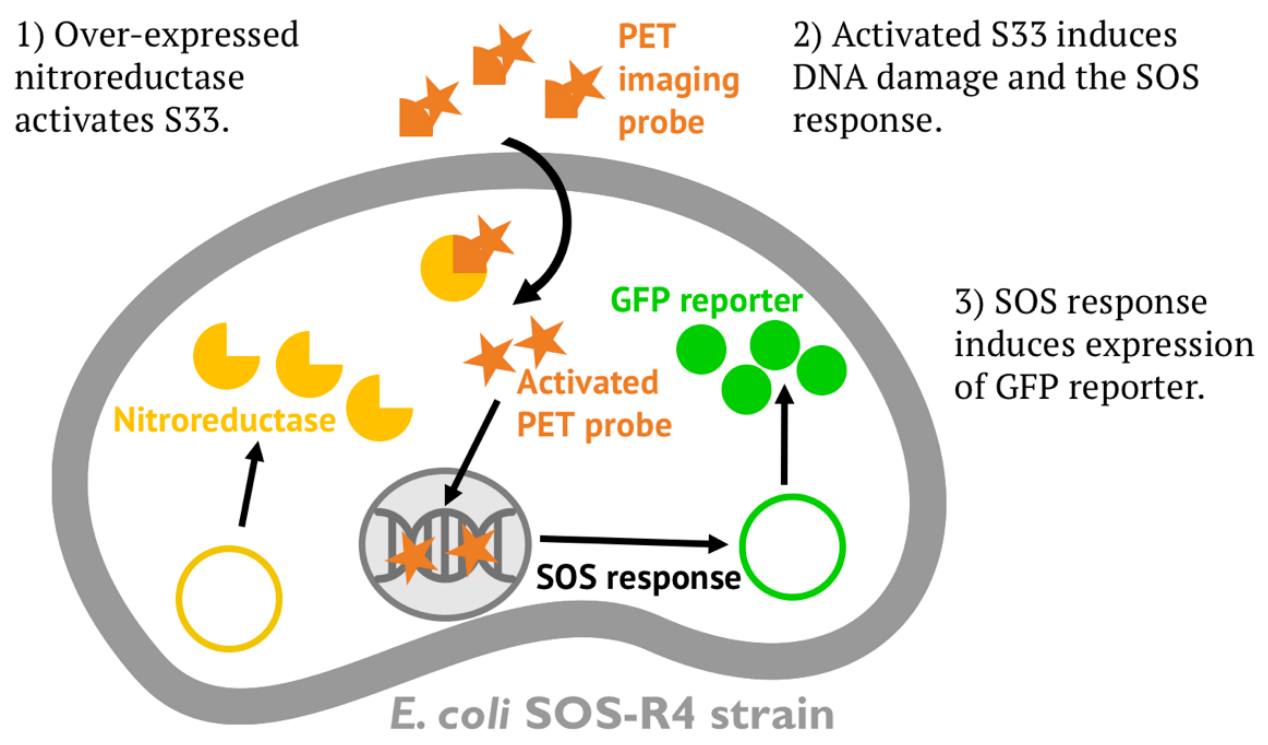

Figure 3-5 Schematic showing the GFP SOS assay in E. coli strain SOS-R4.

\subsubsection{Bacterial nitroreductase expression and stability in human cell lines}

Though long-term stable expression of a bacterial nitroreductase is not required in a human cell in GDEPT applications, as this mimics neither bacterial nor viral delivery vector infection, stably transfected human cell lines are important for cell culture assays and mouse xenograft models to test in vivo activity without requirement of an elaborate vector infection scenario.

However one of the major issues that the Ackerley group has faced is the widely varying degrees of expression of many different nitroreductase candidates following stable transfection in HCT-116 cells, with some failing 
to express at all (Figure 3-6), irrespective of whether the human EF-1 $\alpha$ promoter (Prosser et al., 2010a) or the cytomegalovirus (CMV) early immediate promoter (Prosser et al., 2013) was used to drive gene expression. This difference in expression does not appear to reflect enzyme thermostability (Dr Janine Copp, personal communication).
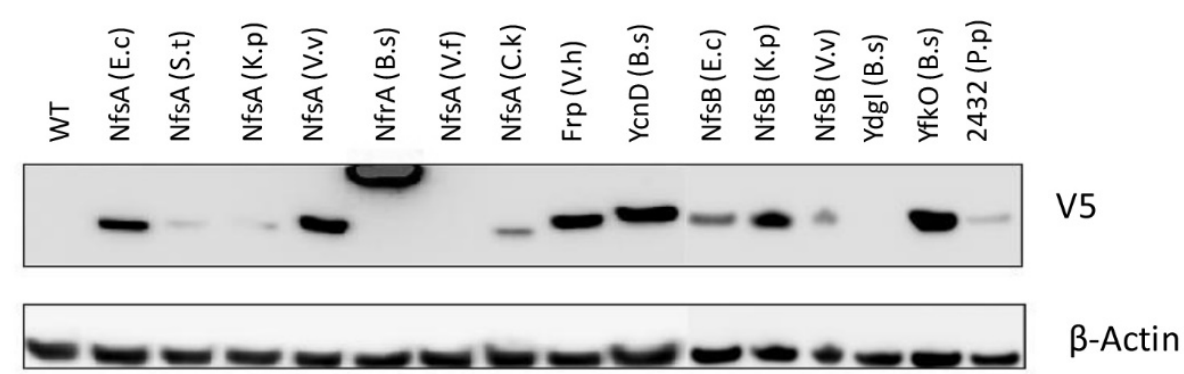

Figure 3-6 Western blot of nitroreductases stably expressed in HCT-116 cell line. Stable long-term expression of 15 wild-type nitroreductase enzymes, recombined with mammalian promoter and enhancer sequences and transfected into HCT116 cells, generated by Dr Gareth Prosser. Nitroreductase expression was analysed by Western blot analysis of V5 tag expression following cell lysis. Reprinted from Prosser et al. (2013) with permission from Elsevier.

We hypothesise that this phenomenon stems from the general promiscuity of nitroreductases, which we postulate could allow for some enzymes to interfere with essential metabolic pathways in human cells to such an extent that their expression is poorly or not at all not tolerated (Prosser et al., 2013; Williams et al., 2015). Therefore, we considered a necessary constraint on selection of nitroreductases for further evolution to improve their activation of S33 was to ensure that the wild-type nitroreductase was capable of stable expression in human cell lines. 


\subsubsection{Nitroreductase variant libraries previously created by the Ackerley group}

Two different nitroreductase variant libraries that had been previously created by the Ackerley group were screened as part of this chapter's attempts to discover variants with improved S33-activating capabilities.

\subsubsection{YcnD_Bs site-saturation mutagenesis (SSM) library}

A SSM library targeting the nitroreductase YcnD from Bacillus subtilis was created by our research group in an attempt to improve enzyme activation of the prodrug PR-104A (Prosser, 2011). A crystal structure of YcnD_Bs with the bound FMN cofactor had been generated by other research groups; however this gave minimal indication as to which active site residues could have roles in NADPH cofactor or substrate stabilisation (Morokutti et al., 2005). In an attempt to select residues that could have roles in cofactor, substrate or active site stabilisation, the active site of YcnD_Bs was superimposed over that of Frp_Vh, an NfsA family enzyme to which it shared 34\% identity with, that had been co-crystallised with a NAD inhibitor (Tanner et al., 1999). Nine different residues in the active site of YcnD_Bs were then selected for individual targeted NNK codon substitution (N: A/C/G/T; K: G/T) which allowed for the coding of all 20 proteinogenic amino acids at a single site (Prosser, 2011). Each resulting YcnD_Bs NNK residue library was cloned into the positive selection vector pUCX:KG, which allowed only vector that had successfully ligated with a desired gene insert to code for an entire kanamycin resistance cassette (Prosser et al., 2015). Five YcnD_Bs SSM active site libraries (at residues I41, G65, R139, Y227, Y233) in the E. coli strain SOS-R2 had been previously screened over the course of my Honours research for S33 activity using $2.5 \mu \mathrm{M}$ S33 and the LacZ SOS assay (results not shown; Rich, 2012). The activities of lead candidates from this screen were analysed further via IC $_{50}$ assays (Table 3-1; Rich, 2012). 
All three leading candidates showed amino acid substitutions at residue Y227, with two variants exhibiting the single Y227G substitution, suggesting that a change at this residue from a bulky Tyr amino acid to a smaller amino acid (Val, Gly, Leu) could allow the active site to more efficiently bind S33 (see modelling of active sites in Figure 3-15). The most active variant, 227_60 (I163V/Y227V), exhibited a 1.6-fold improvement in sensitising SOSR2 to S33 over YcnD_Bs, whereas the single Y227G variant showed a 1.4-fold reduction in $\mathrm{IC}_{50}$ over YcnD_Bs, and 227_35 (Y227L/M239V) demonstrating a 1.3-fold reduction in $\mathrm{IC}_{50}$ (Rich, 2012). The remaining three variants exhibited substitutions in I41 to Met or Trp, which are both hydrophobic residues. Sequencing results revealed that due to the use of a non highfidelity polymerase by Dr Prosser during library construction, some of the variants also had additional amino acid substitutions elsewhere in the gene (as seen in variants 227_60 and 227_35; Rich, 2012).

\begin{tabular}{|c|c|c|c|}
\hline Enzyme & $\mathrm{IC}_{50}(\boldsymbol{\mu} \mathrm{M})$ & $\begin{array}{l}\text { Fold } \\
\text { reduction in } \\
\mathrm{IC}_{50} \text { over } \\
\text { YcnD_Bs }\end{array}$ & $\begin{array}{c}\text { aa } \\
\text { substitutions }\end{array}$ \\
\hline pUCX:KG_YcnD_227_60 & $23.5 \pm 0.7$ & 1.6 & I163V/Y227V \\
\hline pUCX:KG_YcnD_227_24 & $26.4 \pm 5.0$ & 1.4 & Y227G \\
\hline pUCX:KG_YcnD_227_74 & $27.1 \pm 5.0$ & 1.4 & Y227G \\
\hline pUCX:KG_YcnD_227_35 & $28.7 \pm 6.2$ & 1.3 & Y227L/M239V \\
\hline pUCX:KG_YcnD_41_17 & $30.0+2.3$ & 1.2 & $\mathrm{I} 41 \mathrm{~T}$ \\
\hline pUCX:KG_YcnD_41_26 & $31.2 \pm 1.6$ & 1.2 & $\mathrm{I} 41 \mathrm{M}$ \\
\hline pUCX:KG_YcnD_41_68 & $33.3 \pm 1.1$ & 1.1 & D19G/I41T \\
\hline pUCX:KG_YcnD_Bs & $36.6 \pm 2.4$ & 1.0 & \\
\hline Empty pUCX:KG & $>>70$ & & \\
\hline
\end{tabular}

Table 3-1 IC 50 values of $S 33$ mediated growth inhibition of lead variants from SOSR2 YcnD_Bs SSM library in pUCX:KG challenged with up to $70 \mu M$ S33. S33 mediated growth inhibition was monitored by measuring strain turbidity $\left(\mathrm{OD}_{600}\right)$ pre-and post-four hour incubation across a two-fold dilution series of S33, with a top concentration of $70 \mu M$ S33. Percentage growth relative to unchallenged controls was determined and used to calculate the concentration at which 50\% growth inhibition was seen using GraphPad Prism 7.0. Data are the average of 3 biological replicates $\pm S D$. Data duplicated from Rich, (2012). 


\subsubsection{NfsA_Ec SDM library}

In previous Ackerley lab research aiming to improve NfsA_Ec activation of the prodrug PR-104A, ten different individual amino acid substitutions in NfsA_Ec were found to enable this function (I5T, S41Y, E99G, L103M, K222E, R225A, R225G, R225P, F227S, L229V). To allow for the discovery of any synergistic interactions between individually beneficial substitutions, an NfsA_Ec site-targeted library called the SDM library was created (Copp et al., 2017). In this library, at each of the eight different amino acid residues either the wild-type or modified residue could occur, generating a pool of hundreds of possible variants. The library was cloned into pUCX and transformed into the $E$. coli SOS screening strain SOS-R2. From this library, variants were recovered that induced a 25-fold increase in LacZ SOS response over NfsA_Ec following exposure to PR-104A, and sensitised E. coli SOS-R2 to up to 3.8-fold lower concentrations of PR-104A than the wildtype parent enzyme (Copp et al., 2017).

\section{$3.2 \quad$ Aims}

- Identify nitroreductases from the 58 nitroreductase core library that exhibit the ability to activate S33.

- Select nitroreductases known to be capable of stable transfection in HCT-116 cell lines for directed evolution to improve their activity with S33.

- Screen previously generated variant libraries of the nitroreductases selected in Aim 2 for improved activation of S33.

\subsection{Results}

\subsection{1 pUCX high and low-expressing plasmids}

Prior to the discussion of major findings discovered in this chapter, a key problem encountered over the course of the research conducted in this thesis will be briefly summarised. This issue involved the unknowing and 
interchanging use of two different pUCX plasmids - the original highexpressing pUCX (plasmid map shown in Figure 2-1) and a derived lowexpressing pUCX (plasmid map shown in Figure 2-2) -to compare nitroreductase activity levels during over-expression assays. The former pUCX plasmid, created with the inclusion of a nitroreductase gene $a z o R_{-} E c$ in the MCS, was amplified by PCR, restriction enzyme-cleaved and selfligated to form an empty pUCX derivative (in the process introducing two additional restriction enzyme sites for PstI and SphI in the MCS; Figure 3-7; Prosser, (2011)). However the use of a non-high-fidelity DNA polymerase during this process unknowingly introduced 9 base pair mutations into the plasmid backbone of empty pUCX (Figure 3-8 and Table 3-2), and any nitroreductase genes subsequently cloned into this low-expressing pUCX plasmid showed substantially lower levels of enzyme expression (Figure 3-9).

An analysis of the nine base mutations present in low-expressing pUCX (Table 3-2) revealed four occurring in the ampicillin resistance cassette and two in the plasmid backbone, and these were determined unlikely to have an effect on plasmid copy number or gene expression. Of two other mutations present in the lacI repressor gene, the first (2965 C>T) was silent; however the second (2643 $A>G$ ) conferred a S151P substitution in the lac repressor protein. This latter substitution has been shown to both increase binding affinity of the lac repressor for the operator and lower its ability to bind (and be repressed) by IPTG (Swint-Kruse et al., 2003). We postulated this might in turn confer lower levels of IPTG-induced expression of nitroreductases from the plasmid due to higher repression. The final mutation, $1441 \mathrm{~T}>\mathrm{C}$, occurred in the pUC origin of replication, and codes for the RNAII transcript involved in initiation of plasmid replication. This mutation (174 bp into the RNAII transcript) is involved in forming the fourth major loop of the RNAII secondary structure (Tomizawa, 1986), and could potentially affect the secondary structure of RNAII and subsequently its ability to hybridise with template DNA and initiate replication. Therefore a lower copy number of 
this plasmid could also be a contributing factor to differences in nitroreductase enzyme expression.

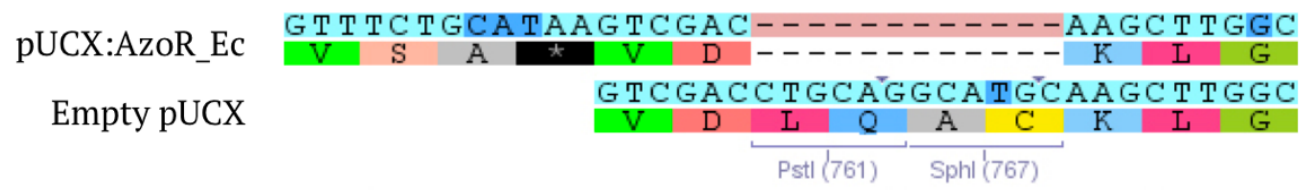

Figure 3-7 Sequence alignment of pUCX:AzoR_Ec and empty $p U C X$. Alignment shows the final three amino acids of AzoR_Ec (VSA) followed by the stop codon and the multiple cloning site downstream in both plasmids. Empty pUCX contains a 12-bp section in the downstream multiple cloning site which is not present in $p U C X: a z o R_{-} E c$; this section contains PstI and SphI restriction enzyme sites.

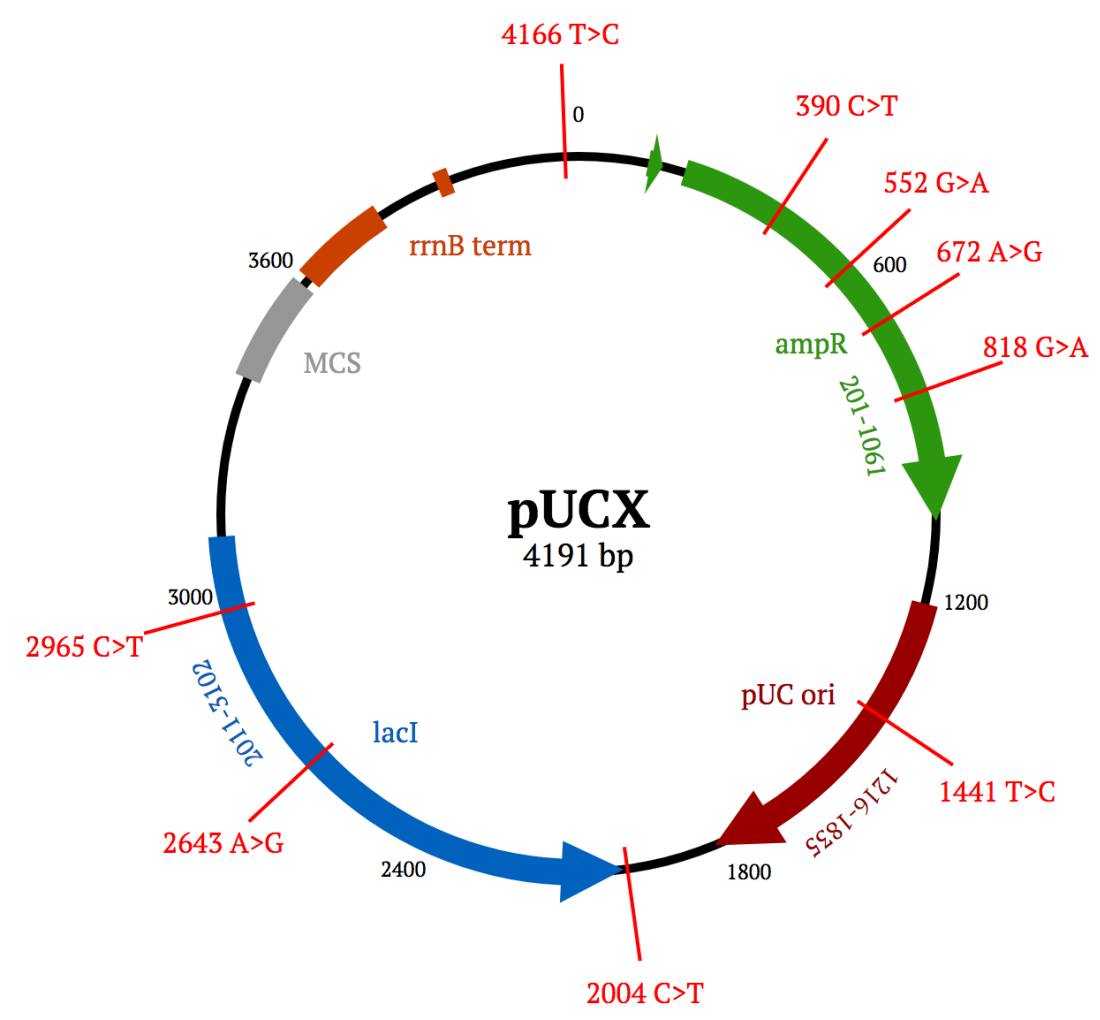

Figure 3-8 Plasmid map and mutations present in expression vector pUCX (lowexpressing). Numbers denote the base pair location of genes of interest. Key features of the plasmid include the Ampicillin resistance cassette (green), pUC ori (red), lacI repressor (blue), multiple cloning site (includes promoter, operator and ribosome binding sites, shown in greater detail in Figure 2-4), and the rrnB terminators (orange). Mutations indicating differences between in lowexpressing $p U C X$ and high-expressing $p U C X$ are labelled in red. 


\begin{tabular}{c|c} 
Base-pair mutation & Gene location of mutation \\
\hline $390 \mathrm{C}>\mathrm{T}$ & $a m p^{R}$ \\
$552 \mathrm{G}>\mathrm{A}$ & $a m p^{R}$ \\
$672 \mathrm{~A}>\mathrm{G}$ & $a m p^{R}$ \\
$818 \mathrm{G}>\mathrm{A}$ & $a m p^{R}$ \\
$1441 \mathrm{~T}>\mathrm{C}$ & $p U C$ ori \\
$2004 \mathrm{C}>\mathrm{T}$ & Plasmid backbone \\
$2643 \mathrm{~A}>\mathrm{G}$ & lacI \\
$2965 \mathrm{C}>\mathrm{T}$ & lacI \\
$4166 \mathrm{~T}>\mathrm{C}$ & Plasmid backbone
\end{tabular}

Table 3-2 Plasmid mutations differentiating pUCX (high-expressing) and pUCX (low-expressing). The location of base pair mutations introduced into $p U C X$ during the creation of pUCX:empty are listed in the left-hand column; the righthand column lists the plasmid genes within which these mutations occurred.

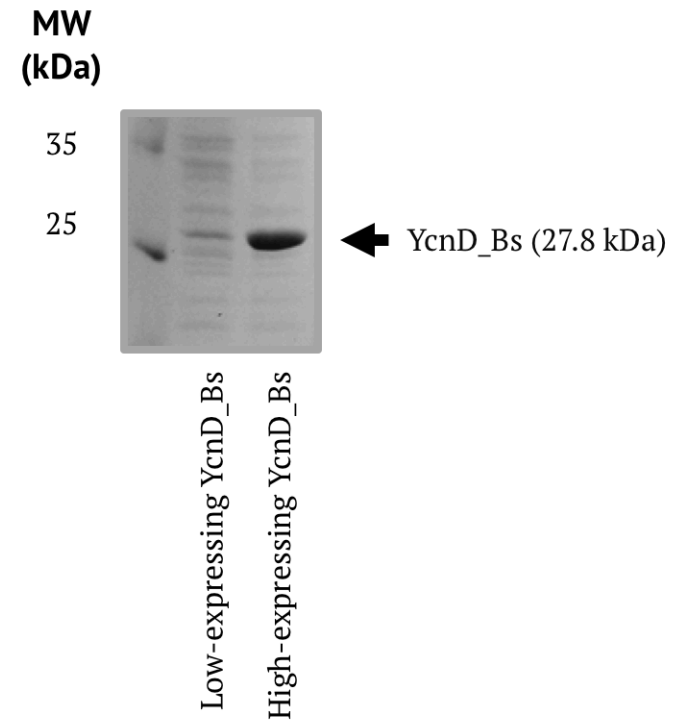

Figure 3-9 SDS-PAGE gel showing low- and high-expressing $p U C X: Y C n D \_B s$ relative protein expression levels from $E$. coli $7 N T$ cells. Nitroreductase over-expression was induced with $50 \mu M$ IPTG for 4 hours prior to sample collection. Samples were normalised for cell density $\left(\mathrm{OD}_{600}\right)$ and $10 \mu \mathrm{L}$ of sample loaded per lane. 


\subsubsection{Difference in expression between low-expressing and high-expressing pUCX}

I was over two years into my $\mathrm{PhD}$ research when I discovered the existence of the lower-expressing variant of pUCX, and hence much of my earlier research was confounded by the comparison of enzyme variants in different plasmid backbones. Consequently, several sets of results in this chapter will be compared against positive controls in both plasmid backbones. (Note: subsequent testing revealed that six nitroreductases NfsA_Es, NemA_St, YwrO_Bs, YwrO_Li, YwrO_Vf and YdjA_Kp from the 58 core nitroreductase library had all been unknowingly cloned into the low-expressing variant of pUCX. The former four variants were later re-cloned into the highexpressing pUCX plasmid).

Once it became apparent that there were two variant forms of pUCX, I sought to quantify the differences in nitroreductase enzyme expression between low-expressing and high-expressing pUCX. To achieve this, E. coli 7NT cells expressing the Bacillus subtilis nitroreductase YcnD (YcnD_Bs) from either low-expressing or high-expressing pUCX were induced with varying concentrations of IPTG $(0 \mu \mathrm{M}$ to $50 \mu \mathrm{M})$. These cells were then subjected to titrated concentrations of the 5-nitroimidazole prodrug metronidazole to determine $\mathrm{IC}_{50}$ values (i.e., the concentration of metronidazole required to reduce growth in culture of a particular E. coli strain to $50 \%$ that of an unchallenged control). High-expressing $\mathrm{YcnD}_{-} \mathrm{Bs}$, when induced with only $5 \mu \mathrm{M}$ IPTG, was found to sensitise 7NT cells to a similar concentration of metronidazole to low-expressing YcnD_Bs induced with the standard $50 \mu \mathrm{M}$ IPTG (Table 3-3), suggesting that levels of overexpressed enzyme were similar across both conditions. 


\begin{tabular}{c|c|c|c}
$\begin{array}{c}\text { IPTG } \\
\text { concentration }\end{array}$ & $\begin{array}{c}\text { Metronidazole IC } 50(\mu \mathrm{M}) \\
\text { HUCX:expressing } \\
\text { pUCXnD_Bs }\end{array}$ & $\begin{array}{c}\text { Low-expressing } \\
\text { pUCX:YcnD_Bs }\end{array}$ & $\begin{array}{c}\text { Empty } \\
\text { pUCX }\end{array}$ \\
\hline $0 \mu$ M IPTG & $51.3 \pm 0.7$ & $>200$ & $>>200$ \\
$2.5 \mu$ M IPTG & $45.4 \pm 4.8$ & $>200$ & $>>200$ \\
$5 \mu$ IPTG & $36.0 \pm 5.0$ & $>200$ & $>>200$ \\
$10 \mu$ IPTG & $13.8 \pm 2.3$ & $101.8 \pm 9.8$ & $>>200$ \\
$25 \mu$ M IPTG & $7.1 \pm 1.4$ & $53.4 \pm 8.1$ & $>>200$ \\
$50 \mu$ M IPTG & $6.7 \pm 0.4$ & $38.8 \pm 5.6$ & $>>200$
\end{tabular}

Table 3-3 IC 50 values of S33 mediated growth inhibition of 7NT bearing highexpressing or low-expressing YcnD_Bs challenged with up to $200 \mu \mathrm{M}$ metronidazole. S33 mediated growth inhibition was monitored by measuring strain turbidity $\left(O D_{600}\right)$ pre- and post-four hour incubation with a two-fold dilution series of metronidazole, with a top concentration of $200 \mu \mathrm{M}$ metronidazole. IPTG concentration used in these assays varied from $0 \mu M$ to 50 $\mu M$. Percentage growth relative to unchallenged controls was determined and used to calculate the concentration at which 50\% growth inhibition was observed using GraphPad Prism 7.0. Data are the average of 3 biological replicates $\pm S D$.

A linear correlation was observed for high-expressing YcnD_Bs and metronidazole $\mathrm{IC}_{50}$ values between IPTG concentrations of $0 \mu \mathrm{M}$ to $10 \mu \mathrm{M}$, suggesting a linear relationship between levels of induced enzyme and the ability of the host strain to reduce metronidazole (Figure 3-10). At IPTG concentrations above $10 \mu \mathrm{M}$ this linear relationship disappeared, suggesting a diminished ability of higher intracellular levels of over-expressed YcnD_Bs to reduce metronidazole as efficiently as lower concentrations. It has been postulated by our lab that this could be due to the inability of $E$. coli cells to continually generate high enough levels of NADPH to keep up with the reaction; however this has not been shown definitively by our group. As a consequence of this discovery, some $\mathrm{IC}_{50}$ assays performed in the research conducted for this thesis utilised lower concentrations of IPTG, to allow for more accurate determination of the activity of improved variants relative to wild-type parent enzymes. 


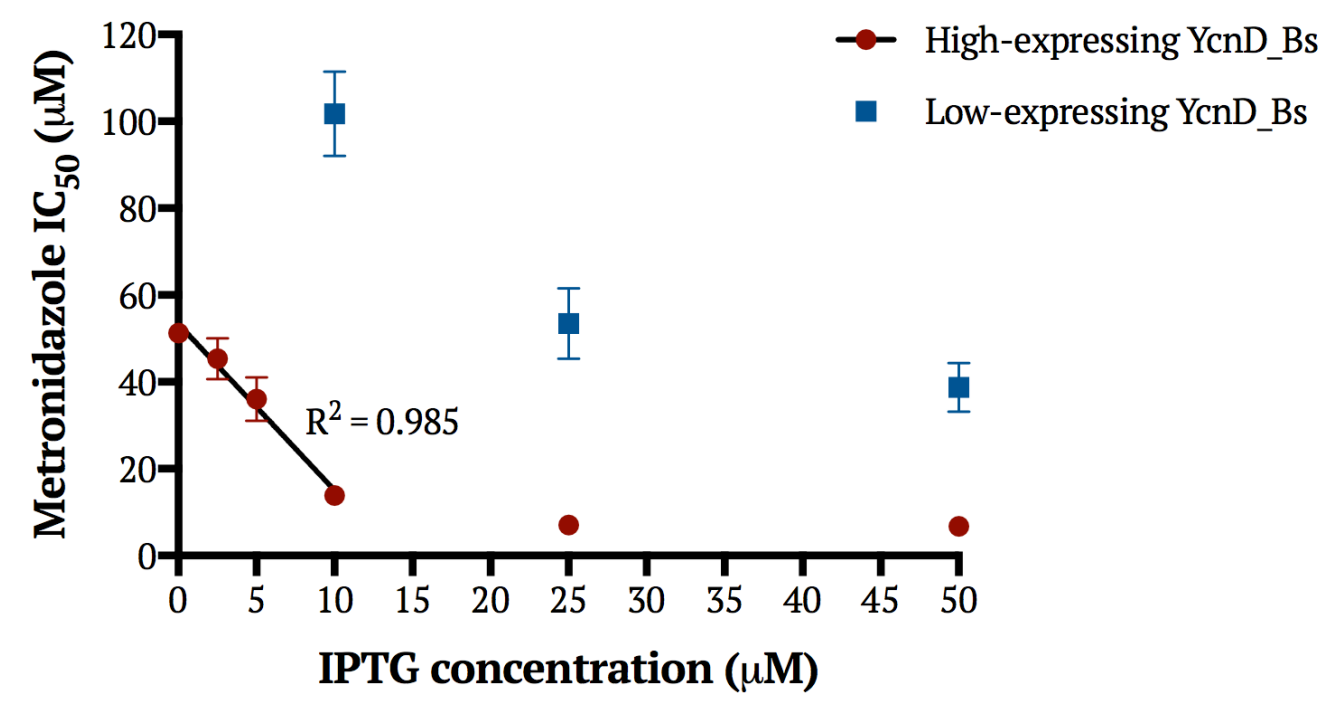

Figure 3-10 Correlation between metronidazole $I C_{50}$ values of low-expressing and high-expressing YCnD_Bs at IPTG concentrations ranging from $0 \mu \mathrm{M}$ to $50 \mu \mathrm{M}$. Data from Table 3-3. A linear correlation for high-expressing YcnD_Bs (red) was observed from $0 \mu M$ IPTG to $10 \mu M$ IPTG, with an $R^{2}$ value of 0.985 .

\subsubsection{Evaluation of wild-type nitroreductase activity with S33}

\subsubsection{GFP SOS assay}

The 58 nitroreductase library in SOS-R4 was screened with sub-lethal concentrations of S33, which allowed for the generation of the GFP SOS response without noticeable growth inhibition of $E$. coli stemming from toxic effects of the reduced S33 metabolites. Following pilot testing across a range of concentrations, final concentrations of S33 of 2.5 and $5 \mu \mathrm{M}$ were selected as yielding a detectable SOS response (Figure 3-11) with minimal growth inhibition (> 75\% growth relative to unchallenged control).

Nearly all members of the NfsA family demonstrated the ability to activate S33, whereas only members of the NfsB family with $<45 \%$ identity to $E$. coli NfsB exhibited activity with S33 (e.g. NfsB_Es, FRaseI_Vf, NfsB_Vh and YfkO_Bs). With the exception of YdgI_Bs from the Nitroreductase 1 family, no nitroreductases from the other families in our library showed any appreciable levels activity with S33. 


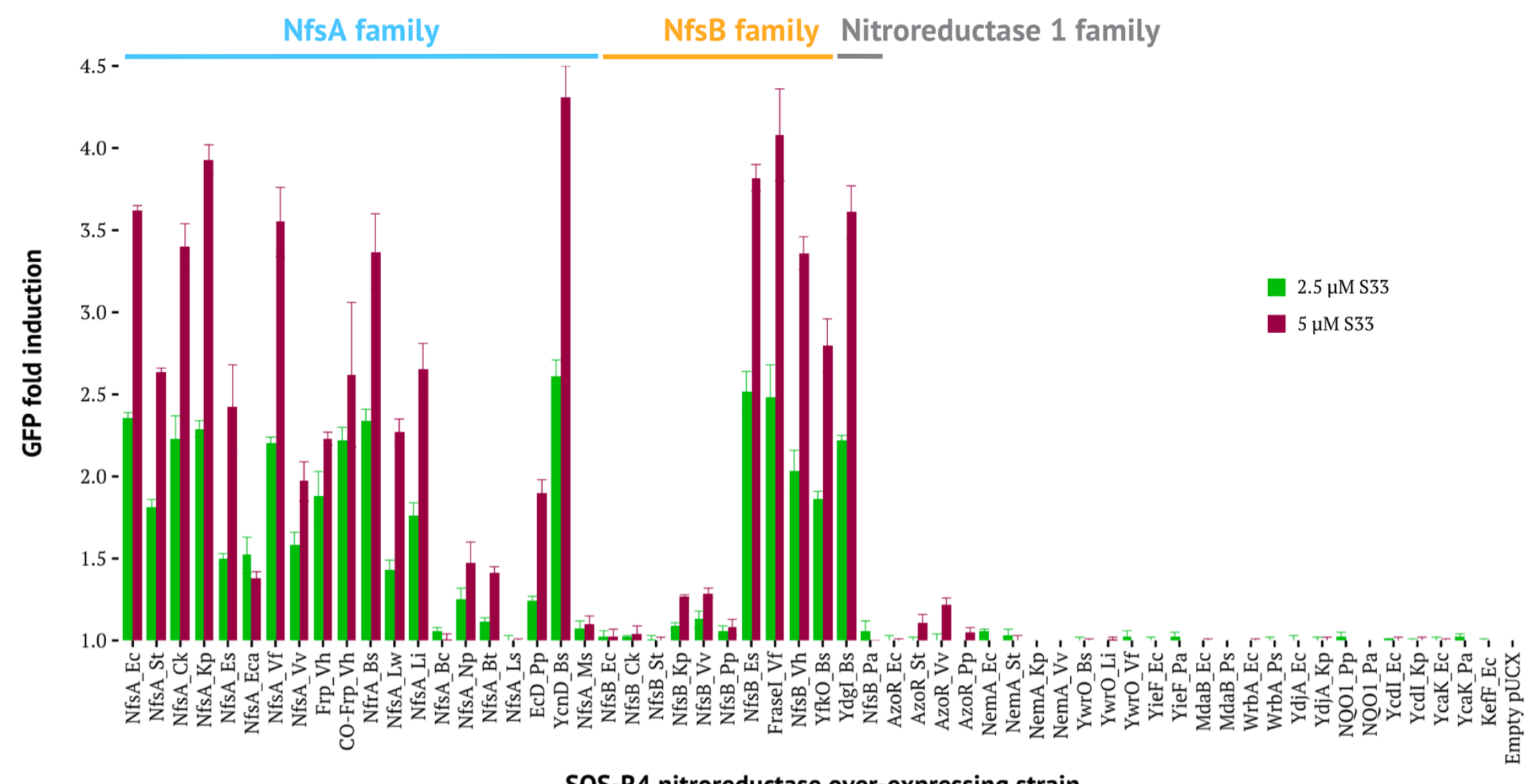

Figure 3-11 S33 mediated induction of the GFP SOS response in E. coli SOS-R4 nitroreductase over-expression strains, challenged with either 2.5 $\mu \mathrm{M}$ or $5 \mu \mathrm{M}$ S33. Over-expression strains were grown in 384-well microplates and challenged with $2.5 \mu M$ and $5 \mu M$ S33 for four hours prior to quantification of SOS response by measurement of GFP fluorescence. Fold induction is derived from the fluorescence recorded on drug exposure divided by that of unchallenged control wells. Data are the average of 3 biological replicates $\pm S E M$. 


\subsubsection{Growth inhibition $\left(I C_{50}\right)$ assays with select candidates from the 58 nitroreductase library and S33}

Nineteen lead nitroreductase candidate enzymes for S33 evolution as determined from the data presented in Figure 3-11 were subjected to more sensitive growth inhibition assays to more accurately assess their S33reducing activity. The determination of $\mathrm{IC}_{50}$ values, which here indicate the concentration of S33 required to reduce growth of a particular nitroreductase over-expressing E. coli strain to $50 \%$ that of an unchallenged control, was conducted in the E. coli 7NT strain by exposure of cells to titrated concentrations of S33 for 4 hours. The 19 candidates included 14 NfsA enzymes, four NfsB enzymes, one Nitroreductase 1 enzyme and an empty pUCX control (Table 3-4). CO-Frp_Vh and Frp_Vh are strain variants of the same enzyme, derived from two different $V$. harveyi isolates; remarkably, the former was found to sensitise E. coli $7 \mathrm{NT}$ cells to a 10 -fold lower concentration of S33 than the latter (Table 3-4).

Of the NfsA enzymes, YcnD_Bs, CO-Frp_Vh and NfsA_Vf were able to induce the lowest $\mathrm{IC}_{50}$ values with $\mathrm{S33}$; over 1.5-fold lower than the most active NfsB variant, NfsB_Es. Of these three NfsA enzymes, YcnD_Bs was the only one known to exhibit stable expression in HCT-116 cells (Figure 3-6); NfsA_Vf was not capable of stable transfection (Figure 3-6; Prosser et al., 2013) and no previous attempts to stably transfect CO-Frp_Vh into human cell lines had been conducted. Of the NfsB enzymes, NfsB_Es, FRaseI_Vf and YfkO_Bs generated the lowest $\mathrm{IC}_{50}$ values with S33; however at the time only YfkO_Bs was known to be capable of stable expression in HCT-116 cells (Figure 3-6; Prosser et al., (2013), Swe at al., (2012); although NfsB_Es was later discovered to also exhibit stable expression in HCT-116 cells; Dr Elsie Williams, personal communication). 


\begin{tabular}{c|c|c}
\multicolumn{1}{c}{ Family } & Enzyme & IC $_{50}(\boldsymbol{\mu M})$ \\
\hline NfsA & YcnD_Bs & $19.4 \pm 1.5$ \\
NfsA & CO-Frp_Vh & $21.0 \pm 4.7$ \\
NfsA & NfsA_Vf & $22.2 \pm 1.5$ \\
NfsA & NfsA_Vv & $26.8 \pm 4.5$ \\
NfsB & NfsB_Es & $33.3 \pm 1.3$ \\
NfsA & NfrA_Bs & $35.9 \pm 3.0$ \\
NfsB & FRaseI_Vf & $36.8 \pm 3.7$ \\
NfsA & NfsA_Ec & $40.0 \pm 1.3$ \\
NfsA & NfsA_St & $43.6 \pm 3.0$ \\
NfsA & NfsA_Ck & $44.9 \pm 3.7$ \\
NfsA & NfsA_Kp & $50.5 \pm 8.3$ \\
NfsB & YfkO_Bs & $52.1 \pm 6.7$ \\
NfsB & NfsB_Vh & $64.3 \pm 9.3$ \\
Nitroreductase 1 & YdgI_Bs & $64.6 \pm 9.5$ \\
NfsA & NfsA_Li & $119 \pm 11$ \\
NfsA & Frp_Vh & $213 \pm 19$ \\
NfsA & NfsA_Lw & $265 \pm 30$ \\
NfsA & EcD_Pp & $279 \pm 26$ \\
NfsA & NfsA_Es & $>300$ \\
& Empty pUCX & $>>300$
\end{tabular}

Table 3-4 IC 50 values of S33 mediated growth inhibition of 19 nitroreductases from the 7NT E. coli 58 nitroreductase over-expression library. S33-dependent growth inhibition was monitored by measuring strain turbidity $\left(O D_{600}\right)$ pre- and postfour hour incubation with a two-fold dilution series of S33, with a top concentration of $300 \mu \mathrm{MS} 33$ to conserve drug stocks. Percentage growth relative to unchallenged controls was determined and used to calculate the concentration at which 50\% growth inhibition was seen using GraphPad Prism 7.0. Data are the average of 3 biological replicates $\pm S D$. Enzymes are ranked in order of their ability to sensitise cells to S33.

\subsubsection{Correlation of GFP SOS response and growth inhibition}

Previous linear-logarithmic correlations conducted between nitroreductaseinduced LacZ SOS responses and $\mathrm{IC}_{50}$ values of SOS-R2 strains expressing the same nitroreductases showed strong correlations following challenge with CB1954 $\left(R^{2}=0.74\right)$ and PR-104A $\left(R^{2}=0.63\right)$ (Prosser, 2011). A correlation between S33-induced GFP responses in SOS-R4 and $\mathrm{IC}_{50}$ values in 7NT strains was conducted to see if similar correlations could be observed (Figure 3-12). 


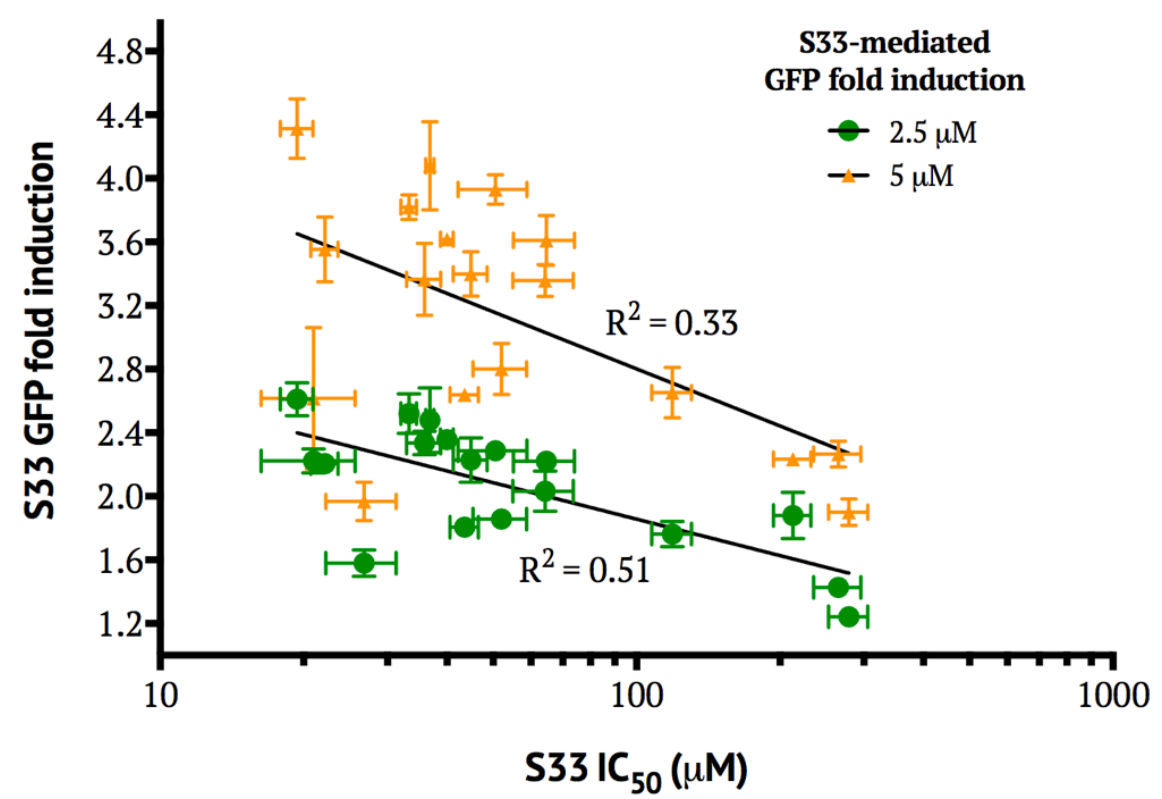

Figure 3-12 Linear-logarithmic correlation between GFP SOS response induced by $S 33$ in SOS-R4 nitroreductase over-expression strains and I $C_{50}$ growth inhibitory effects of S33 in 7NT nitroreductase over-expression strains. Response of 19 nitroreductases to $5 \mu M$ (orange) and $2.5 \mu M$ (green) S33 in the GFP SOS assay (data from Figure 3-11) and up to $300 \mu \mathrm{M} \mathrm{S33}$ in IC $\mathrm{C}_{50}$ assays (data from Table 3-4).

These results indicated only a slight to moderate correlation between $\mathrm{IC}_{50}$ values and GFP response induction at $2.5 \mu \mathrm{M} \mathrm{S33}\left(\mathrm{R}^{2}=0.51\right)$ and $5 \mu \mathrm{M}$ S33 $\left(\mathrm{R}^{2}=0.33\right)$ between the two $E$. coli strains, suggesting that the GFP signal might only provide a rough estimation of the activity of enzymes with S33.

\subsubsection{Glucose oxidase-catalase oxygen scavenging for kinetics}

Confirmation of nitroreductase activity with S33 in vitro was sought via evaluation of S33 reduction by purified $\mathrm{His}_{6}$-tagged nitroreductases. Kinetics were performed with two NfsA enzymes, YcnD_Bs and NfsA_Ec (the latter having a 2-fold higher $\mathrm{IC}_{50}$ with $\mathrm{S} 33$ than YcnD_Bs, similar to the $\mathrm{IC}_{50}$ of cells over-expressing YfkO_Bs), and the NfsB enzymes YfkO_Bs, NfsB_Es, and NfsB_Ec (the latter having no activity with S33 in the GFP SOS assay; Figure 3-11). 
Because nitroreductases are dual-substrate oxidoreductases that exhibit a ping pong bi-bi kinetic mechanism, the rate of reduction of a substrate will vary at different concentrations of $\mathrm{NAD}(\mathrm{P}) \mathrm{H}$, hence we describe apparent kinetic parameters (apparent $K_{M}$ and $k_{c a t}$ ) at the specific NADPH concentration used in the reaction (the $k_{c a t} / K_{M}$ ratio however is not affected by varying $\mathrm{NAD}(\mathrm{P}) \mathrm{H}$ concentrations).

Rates of reduction were monitored at a $350 \mathrm{~nm}$ wavelength (at which both the oxidation of NADPH and reduction of S33 contributed to a decrease in absorbance) for the purpose of this research. At this wavelength, initial rates of reduction of a combination of $250 \mu \mathrm{M}$ NADPH and up to $1200 \mu \mathrm{M}$ S33 could be accurately monitored in a $2 \mathrm{~mm}$ pathlength Uvette.

Previous attempts to generate kinetic data with S33 have had limited success, most likely due to the very low turnover $\left(k_{c a t}\right)$ of $\mathrm{S} 33$ by $\mathrm{His}_{6}$-purified nitroreductases from our library. We and others have found this to be typical of 5-nitroimidazole substrates in general, including metronidazole (Olekhnovich et al., 2009; Williams, 2013), however it is problematic for kinetic measurements owing to the low level background oxidase activity (direct electron transfer from $\mathrm{NADPH}$ to dissolved $\mathrm{O}_{2}$ in the reaction media, measured as the amount of enzyme-catalysed NADPH depletion observed in the absence of any additional substrate). This activity is characteristic of all nitroreductases, in particular NfsA family members, and confounds reaction measurements based on NADPH consumption for slow-turnover substrates (Williams, 2013).

Thus to allow for the measurement of S33 reduction, particularly at low concentrations of the probe, a glucose oxidase-catalase oxygen scavenging system modified from that described by Olekhnovich et al., (2009) was utilised. In this system, glucose oxidase enzyme added to the reaction system converts water, oxygen and added D-glucose ultimately to hydrogen peroxide and gluconic acid (Figure 3-13; Englander et al., 1987). The hydrogen peroxide is converted back to water by added catalase enzyme to 
minimise build-up of reaction oxygen species in the solution (Figure 3-13;

Englander et al., 1987).

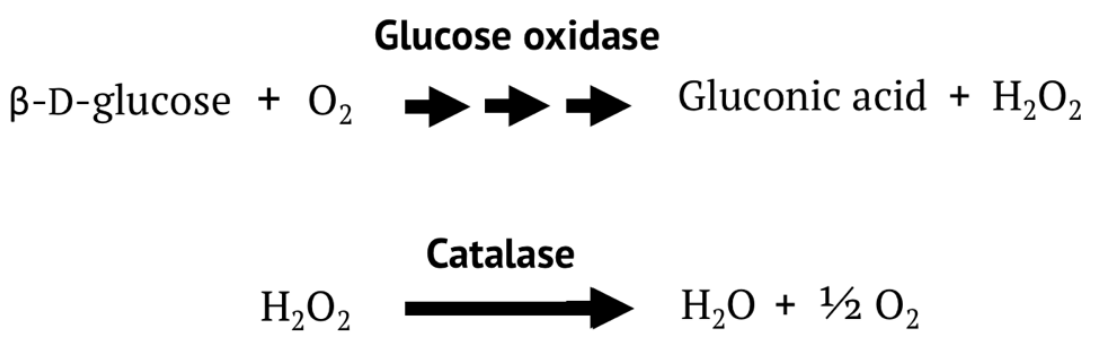

Figure 3-13 Mechanism of action of glucose oxidase-catalase oxygen scavenging system. Glucose oxidase catalyses the conversion of $\beta$ - $D$-glucose and oxygen to gluconic acid and hydrogen peroxide. Catalase catalyses the conversion of hydrogen peroxide to water and oxygen.

\subsubsection{Validation of the glucose oxidase-catalase oxygen scavenging system using CB1954 as a control substrate}

To confirm that the oxygen-scavenging system was not interfering significantly with nitroreductase-catalysed reduction of nitroaromatic compounds, control assays were conducted using $0.280 \mu \mathrm{M} \mathrm{His}$-purified $_{6}$ NfsA_Ec, $250 \mu \mathrm{M}$ NADPH and varying concentrations of CB1954 (up to 800 $\mu \mathrm{M}$ ) with and without the presence of a glucose oxidase-catalase oxygen scavenging system (Figure 3-14). Changes in absorbance (due to formation of reduced CB1954 products) were monitored at $420 \mathrm{~nm}$ wavelength, a wavelength at which both the 2-hydroxylamine and 4-hydroxylamine products of CB1954 absorb equally (Race et al., 2007).

No significant differences were noted between apparent $k_{\text {cat }}(\mathrm{p}$ value $=0.68$ ), $K_{M}(\mathrm{p}$ value $=0.18)$ or $k_{\text {cat }} / K_{M}(\mathrm{p}$ value $=0.20)$ parameters in the presence or absence of the glucose oxidase-catalase oxygen scavenging system (Table 3-5) when compared using Student's t-test. In both cases the $k_{\text {cat }} / K_{M}$ was similar to that previously reported by our group (Column 3 of Table 3-5; Prosser et al., 2013). We were thus satisfied that the oxygen-scavenging system did not substantially impair measurement of enzyme kinetic 
parameters with CB 1954, and proceeded to use this system for the determination of kinetic parameters of nitroreductase enzymes with S33.

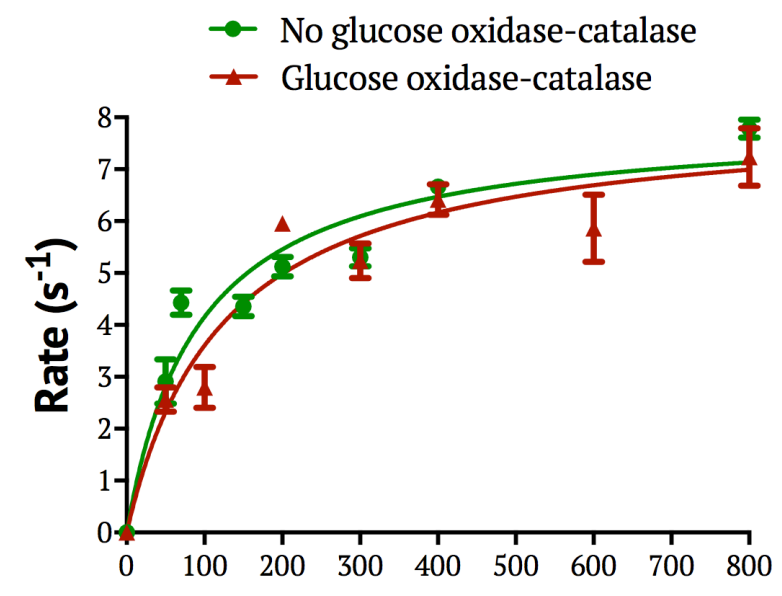

CB $1954(\mu \mathrm{M})$

Figure 3-14 Kinetic parameters for the reduction of CB1954 by purified (His $6^{-}$ tagged) NfsA_Ec. Initial rates of reaction with varying concentrations of CB1954 (0-200 $\mu M)$ and $250 \mu M N A D P H$, with and without the glucose oxidase-catalase oxygen scavenging system (12 units. $m L^{-1}$ glucose oxidase, 12 units. $m L^{-1}$ catalase, $50 \mathrm{mM}$ glucose). At each CB1954 concentration rates were measured in at least triplicate and errors represent $\pm S D$.

\begin{tabular}{|c|c|c|c|}
\hline & $\begin{array}{c}\text { No glucose } \\
\text { oxidase-catalase } \\
\text { oxygen } \\
\text { scavenging } \\
\text { system }\end{array}$ & $\begin{array}{l}\text { Glucose-oxidase- } \\
\text { catalase oxygen } \\
\text { scavenging system }\end{array}$ & $\begin{array}{c}\text { Kinetic } \\
\text { parameters } \\
\text { generated by } \\
\text { Prosser et al., } \\
\text { (2013) }\end{array}$ \\
\hline $\boldsymbol{k}_{\text {cat }}\left(\mathbf{s}^{-1}\right)$ & $7.9 \pm 0.5$ & $8.1 \pm 0.6$ & 16 \\
\hline$K_{M}(\mu \mathrm{M})$ & $91 \pm 18$ & $124 \pm 30$ & 220 \\
\hline$k_{\text {cat }} / K_{M}\left(M^{-1} \cdot s^{-1}\right)$ & $87,000 \pm 18,000$ & $65,000 \pm 17,000$ & $73,000 \pm 6,400$ \\
\hline
\end{tabular}

Table 3-5 Michaelis-Menten kinetic parameters for NfSA_Ec and CB1954. Initial rates of reduction at varying CB1954 concentrations and a fixed concentration of excess NADPH $(250 \mu \mathrm{M})$ were monitored at $350 \mathrm{~nm}$, with rates at each CB1954 concentration measured in at least triplicate. Apparent $K_{M}$ and $k_{\text {cat }}$ at $250 \mu \mathrm{M}$ $N A D P H$ with and without a glucose oxidase-catalase oxygen scavenging system (12 units. $\mathrm{mL}^{-1}$ glucose oxidase, 12 units. $\mathrm{mL}^{-1}$ catalase, $50 \mathrm{mM}$ glucose) were calculated using GraphPad 7.0. An extinction coefficient of $1,200 \mathrm{M}^{-1} \mathrm{~cm}^{-1}$ was used (Prosser et al., 2010a) and errors represent \pm SEM. Parameters in Column 3 were duplicated from Prosser et al, (2013) and represent apparent $K_{M}$ and $k_{\text {cat }}$ at $250 \mu M N A D P H$ in quadruplicate reactions. 


\subsubsection{Reduction of S33 using the glucose oxidase-catalase oxygen scavenging system and His $_{6}-$ purified nitroreductase proteins}

Kinetics for purified nitroreductases with $\mathrm{S} 33$ were measured at $350 \mathrm{~nm}$, using an extinction coefficient of $9650 \mathrm{M}^{-1} \mathrm{~cm}^{-1}$ to allow for the combined effects of NADPH oxidation and S33 reduction (see Section 2.13.1 for details regarding calculation of extinction coefficient). Low-level background NADPH oxidase activity was still observed in the presence of the glucose-oxidase catalase oxygen-scavenging system, and the decrease in absorbance in the absence of S33 was subtracted from readings generated in the presence of S33.

\begin{tabular}{|c|c|c|c|c|}
\hline Enzyme & $\boldsymbol{k}_{\text {cat }}\left(\mathbf{s}^{-1}\right)$ & $K_{M}(\mu \mathrm{M})$ & $\begin{array}{c}\boldsymbol{k}_{c a t} / K_{M} \\
\left(\mathbf{s}^{-1} \cdot \mathbf{M}^{-1}\right)\end{array}$ & $\begin{array}{c}\text { NADPH oxidase } \\
\text { activity }(\mu \mathrm{M} \\
\text { NADPH. } \mu \mathrm{M} \\
\left.\text { enzyme }^{-1} . \mathrm{s}^{-1}\right)\end{array}$ \\
\hline YcnD_Bs & $0.47 \pm 0.03$ & $570 \pm 80$ & $820 \pm 130$ & $0.47 \pm 0.12$ \\
\hline YfkO_Bs & $2.7 \pm 1$ & $4800 \pm 2400$ & $560 \pm 350$ & $0.12 \pm 0.00$ \\
\hline NfsA_Ec & $2.0 \pm 0.4$ & $3700 \pm 1100$ & $530 \pm 200$ & $0.22 \pm 0.04$ \\
\hline NfsB_Es & $2.1 \pm 0.8$ & $5000 \pm 2600$ & $420 \pm 270$ & $0.05 \pm 0.00$ \\
\hline NfsB_Ec & ND & ND & ND & ND \\
\hline
\end{tabular}

Table 3-6 Michaelis-Menten kinetic parameters estimated for nitroreductases and S33. Initial rates of reduction at varying $S 33$ concentrations and a fixed concentration of excess NADPH $(250 \mu \mathrm{M})$ were monitored at $350 \mathrm{~nm}$, with rates at each $S 33$ concentration measured in at least triplicate. Apparent $K_{M}$ and $k_{\text {cat }}$ at $250 \mu \mathrm{MNADPH}$ with a glucose oxidase-catalase oxygen scavenging system (12 units. $\mathrm{mL}^{-1}$ glucose oxidase, 12 units. $\mathrm{mL}^{-1}$ catalase, $50 \mathrm{mM}$ glucose) were calculated using GraphPad 7.0. An extinction coefficient of $9,650 \mathrm{M}^{-1} \mathrm{~cm}^{-1}$ was used and errors represent \pm SEM. NADPH oxidase activity shows enzymemediated NADPH oxidation in the absence of a glucose oxidase-catalase oxygen scavenging system, with an extinction coefficient of $4020 \mathrm{M}^{-1} \mathrm{~cm}^{-1}$ used.

YcnD_Bs showed the most activity with S33, with at least a 6-fold lower apparent $K_{M}$ than other nitroreductases and the highest $k_{\text {cat }} / K_{M}$. YfkO_Bs, NfsB_Es and NfsA_Ec all exhibited higher apparent turnover rates $\left(k_{c a t}\right)$ than YcnD_Bs, but also much higher apparent $K_{M}$ values, and NfsB_Ec demonstrated no apparent activity with S33. The calculated $k_{\text {cat }} / K_{M}$ ratios are very low, which may reflect inaccuracies in estimating the extinction 
coefficient; nevertheless the relative ratios should be indicative of relative levels of enzyme activity (and hence useful for monitoring improvement of directed evolution as well).

Having established that we in vitro kinetic parameters for purified nitroreductase enzymes could be measured in a manner that was consistent with their in vivo activities (as determined by SOS and $\mathrm{IC}_{50}$ assays), I next sought to screen mutant libraries of YcnD_Bs and NfsA_Ec previously created by the Ackerley lab group (see Sections 3.1.6.1 and 3.1.6.2) as a possible source of improved S33-activating variants.

\subsubsection{Screening of YcnD_Bs SSM library for activity with S33}

\subsubsection{I $C_{50}$ analysis of YCnD_Bs SSM library variants with S33}

As the pUCX:KG plasmid developed by Dr Prosser was already known to express cloned genes at lower levels than pUCX (Prosser et al, 2015), to enable direct comparison of the activities of these variants with YcnD_Bs mutants generated subsequently through error-prone PCR directed evolution (Chapter 4), all variants were cloned into pUCX and transformed into the 7NT strain, for repeat $\mathrm{IC}_{50}$ assays. Simultaneously, overlap PCR was used to generate single site derivatives of the YcnD variants that contained double amino acid substitutions, to determine which of the substitutions had the highest direct effect on improvements in enzyme activity with S33. $\mathrm{IC}_{50}$ assays were performed with all YcnD_Bs single- and double-site variants in pUCX in E. coli 7NT cells (Table 3-7). Due to the different levels of expression between YcnD_Bs in high-expressing pUCX relative to pUCX:KG, the IPTG concentration was decreased from $50 \mu \mathrm{M}$ to $10 \mu \mathrm{M}$, empirical testing having indicated that this would allow the generation of similar IC $_{50}$ values to those measured with the pUCX:KG constructs (results not shown). 


\begin{tabular}{|c|c|c|c|}
\hline Enzyme & $I C_{50}(\mu \mathrm{M})$ & $\begin{array}{c}\text { Fold reduction } \\
\text { in } I C_{50} \text { over } \\
\text { YcnD_Bs }\end{array}$ & $\begin{array}{c}\text { aa } \\
\text { substitutions }\end{array}$ \\
\hline pUCX:YcnD 227_60 & $15.3 \pm 3.0$ & 2.0 & I163V/Y227V \\
\hline pUCX:YcnD_Y227L & $16.9 \pm 2.7$ & 1.8 & Y227L \\
\hline pUCX:YcnD_Y227V & $17.8 \pm 4.6$ & 1.7 & Y227V \\
\hline pUCX:YcnD_227_35 & $18.6 \pm 3.4$ & 1.7 & Y227L/M239V \\
\hline pUCX:YcnD_227_24 & $19.5 \pm 3.7$ & 1.6 & Y227G \\
\hline pUCX:YcnD_41_26 & $20.6 \pm 4.4$ & 1.5 & $\mathrm{I} 41 \mathrm{M}$ \\
\hline pUCX:YcnD_41_17 & $26.4 \pm 5.5$ & 1.2 & $\mathrm{I} 41 \mathrm{~T}$ \\
\hline pUCX:YcnD_I163V & $29.6 \pm 4.6$ & 1.0 & I163V \\
\hline pUCX:YcnD_41_68 & $30.1 \pm 4.3$ & 1.0 & D19G/I41T \\
\hline pUCX:YcnD_Bs & $30.9 \pm 5.4$ & 1.0 & - \\
\hline pUCX:YcnD_M239V & $33.1 \pm 6.5$ & 0.9 & M239V \\
\hline pUCX:YcnD_D19G & $37.2 \pm 7.7$ & 0.8 & D19G \\
\hline Empty pŪCX & >> 100 & & \\
\hline
\end{tabular}

Table 3-7 IC 50 values for S33 mediated growth inhibition of lead variants of YcnD_Bs in E. coli 7NT challenged with up to $100 \mu \mathrm{M}$ S33. S33 mediated growth inhibition was monitored by measuring strain turbidity $\left(\mathrm{OD}_{600}\right)$ pre- and postfour hour incubation across a two-fold dilution series of S33, with a top concentration of $100 \mu \mathrm{M}$. The IPTG concentration used in these assays was 10 $\mu M$. Percentage growth relative to unchallenged controls was determined and used to calculate the concentration at which 50\% growth inhibition was observed using GraphPad Prism 7.0. Data are the average of 3 biological replicates $\pm S D$.

Fold reductions in $\mathrm{IC}_{50}$ values of $7 \mathrm{NT}$ cells bearing pUCX YcnD variants over wild-type pUCX:YcnD_Bs (Table 3-7) were very similar to those seen in the pUCX:KG plasmid backbone (Table 3-1). The variant 227_60 (I163V/Y227V) again showed the highest activity with $\mathrm{S33}$, this time with a 2 -fold reduction in $\mathrm{IC}_{50}$ over YcnD_Bs. When the single amino acid substitutions were tested individually, Y227V demonstrated a 1.7 -fold reduction in $\mathrm{IC}_{50}$ over wild type YcnD_Bs, whereas the I163V substitution on its own showed no improvement in sensitising E. coli to S33 over WT YcnD_Bs. This suggested that the Y227V substitution was the main contributor to improvement in S33 activity, but I163V may also have contributed synergistically to improvements in enzyme activity. With the variant 227_35 (Y227L/M239V), the Y227L substitution on its own demonstrated a similar ability to sensitise E. coli to S33 as the double mutant, whereas the M239V substitution on its own showed no improvement over YcnD_Bs, suggesting that the latter was a 
passenger mutation only. The single Y227G substitution (variant 227_24) on its own conferred a 1.6-fold reduction in $\mathrm{IC}_{50}$ over YcnD_Bs. Thus, in all cases it was the residue intentionally targeted by Dr Prosser that yielded the improved nitroreductase activity.

\subsubsection{Active site modelling for selected YcnD Y227 mutants}

A

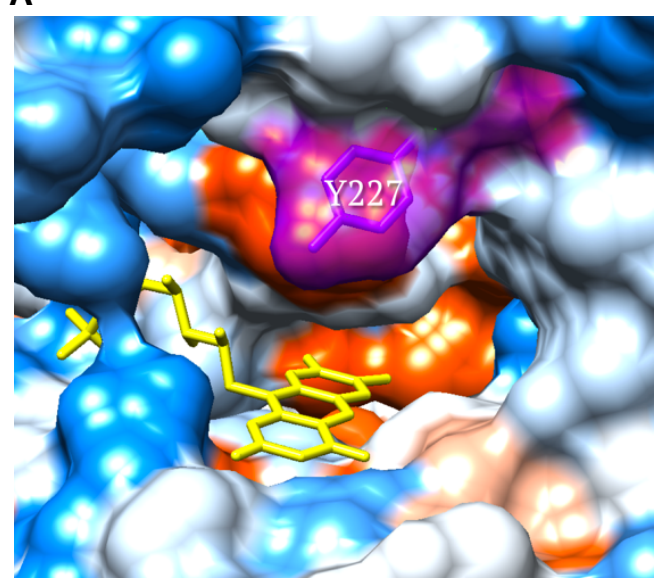

B

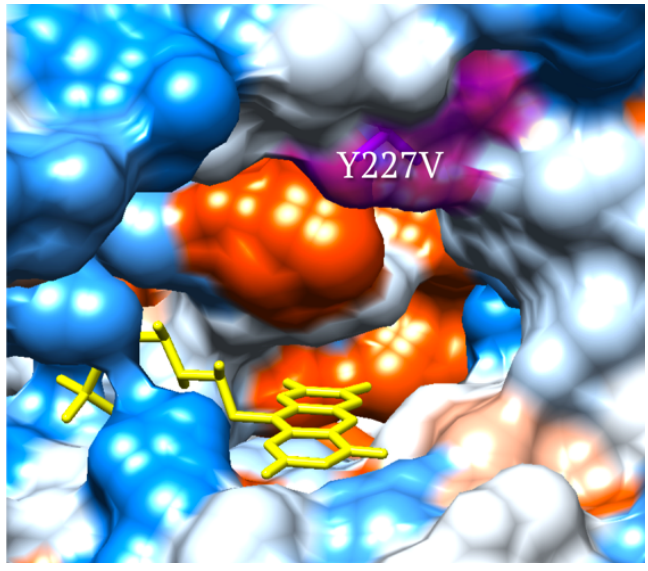

C

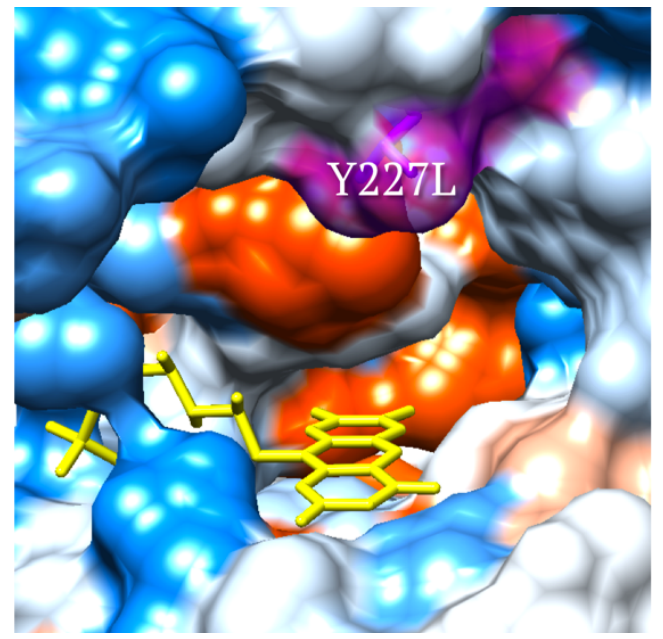

D

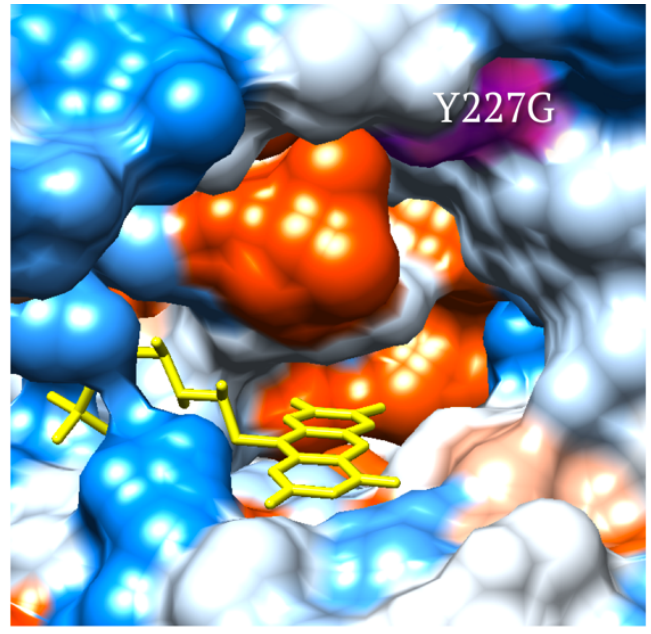

Figure 3-15 Modelling of active site of YCnD_Bs and three site-targeted variants. A) Modelling of Tyr 227 in active site of YcnD_Bs. Tyr 227 is coloured purple. The FMN molecule is coloured yellow. B) Modelling of active site of YcnD variant 227_60 (I163V/Y227V, only Y227V is in active site). C) Modelling of active site of YcnD variant 227_35 (Y227L/M239V, only Y227L is in active site). D) Modelling of active site of YcnD variant 227_24 (Y227G). 
Our modelling of the effects of amino acid substitutions at Y227 (Figure 3-15) suggested that substitutions of Val, Leu or Gly in place of Tyr at this position could allow for an enlarged enzyme active site and thereby facilitate the docking of S33.

\subsubsection{Reduction of S33 by purified His ${ }_{6}$-tagged purified YcnD variant proteins}

The YcnD_Bs variants 227_60, 227_35 and 227_24 were re-cloned into pET28a+ in the E. coli BL21 strain to allow His $_{6}$-tagged protein purification for the determination of their kinetic parameters with S33.

\begin{tabular}{|c|c|c|c|c|}
\hline Enzyme & $\boldsymbol{k}_{\text {cat }}\left(\mathbf{s}^{-1}\right)$ & $K_{M}(\mu \mathrm{M})$ & $\begin{array}{c}\boldsymbol{k}_{\text {cat }} / K_{M} \\
\left(\mathbf{s}^{-1} \cdot \mathbf{M}^{-1}\right)\end{array}$ & $\begin{array}{c}\text { NADPH } \\
\text { oxidase } \\
\text { activity }(\mu \mathrm{M} \\
\text { NADPH. } \mu \mathrm{M} \\
\text { enzyme }^{-1} \cdot \mathrm{s}^{-1} \text { ) }\end{array}$ \\
\hline YcnD I163V/Y227V & $1.0 \pm 0.07$ & $380 \pm 80$ & $2,600 \pm 600$ & $0.24 \pm 0.03$ \\
\hline YcnD Y227L/ M239V & $0.82 \pm 0.12$ & $2600 \pm 600$ & $320 \pm 90$ & $0.07 \pm 0.00$ \\
\hline YcnD Y227G & $0.22 \pm 0.02$ & $470 \pm 90$ & $470 \pm 100$ & $0.13 \pm 0.01$ \\
\hline YcnD_Bs & $0.47 \pm 0.03$ & $570 \pm 80$ & $820 \pm 130$ & $0.47 \pm 0.12$ \\
\hline
\end{tabular}

Table 3-8 Michaelis-Menten kinetic parameters for YcnD variants and S33. Initial rates of reduction at varying $S 33$ concentrations and a fixed concentration of excess NADPH $(250 \mu \mathrm{M})$ were monitored at $350 \mathrm{~nm}$, with rates at each $S 33$ concentration measured in at least triplicate. Apparent $K_{M}$ and $k_{\text {cat }}$ at $250 \mu \mathrm{M}$ NADPH with a glucose oxidase-catalase oxygen scavenging system (12 units. $\mathrm{mL}^{-}$ ${ }^{1}$ glucose oxidase, 12 units. $\mathrm{mL}^{-1}$ catalase, $50 \mathrm{mM}$ glucose) were calculated using GraphPad 7.0. An extinction coefficient of 9,650 $\mathrm{M}^{-1} \mathrm{~cm}^{-1}$ was used and errors represent \pm SEM. NADPH oxidase activity shows enzyme-mediated NADPH oxidation in the absence of a glucose oxidase-catalase oxygen scavenging system, with an extinction coefficient of $4020 \mathrm{M}^{-1} \mathrm{~cm}^{-1}$ used.

Variant 227_60 (I163V/Y227V) showed a 3.2-fold increase in $k_{\text {cat }} / K_{M}$ with S33 over WT YcnD_Bs. The other two variants 227_35 (Y227L/M239V) and Y227G both exhibited worse $k_{\text {cat }} / K_{M}$ than YcnD_Bs, which belied their demonstrated in vivo activity. However, these variants also exhibited a 3-6 fold lower NADPH oxidase activity, and it was postulated that their 
improved activity with S33 in vivo could be due either to their increased relative affinity for $\mathrm{S} 33$ over other substrates present in $E$. coli.

\subsubsection{SOSR2 NfsA_Ec SDM library screening for S33 activity}

\subsubsection{Screening of Nfs__Ec SDM library using the LacZ SOS assay}

From the NfsA_Ec SDM library, 1750 clones were screened for S33-activating variants using the LacZ SOS assay (across 19 96-well plates; results not shown). Each plate was screened once (two technical replicates) in the E. coli SOS-R2 strain using $2.5 \mu \mathrm{M} \mathrm{S33}$, and 57 promising mutants (three from each library plate) were selected for second-tier LacZ SOS assay screening in a single 96-well microplate, again with $2.5 \mu \mathrm{M}$ S33 (Figure 3-16).

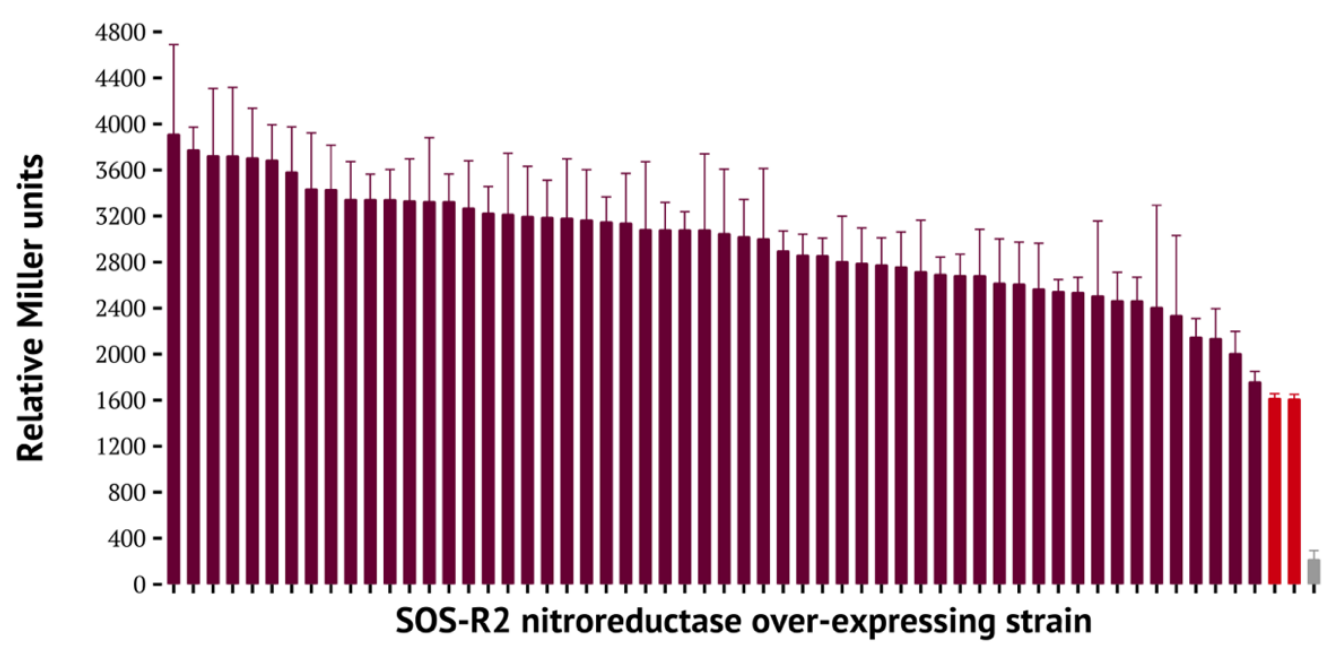

Figure 3-16 Second-tier screening of S33 mediated induction of LacZ SOS response in $E$. coli SOS-R2 nitroreductase over-expressing strains challenged with $2.5 \mu \mathrm{M}$ S33. Over-expression strains were grown in 96-well microplates and challenged with $2.5 \mu M S 33$ for 4 hours prior to quantification of SOS response by $\beta$ galactosidase assay. Relative Miller units were derived from subtraction of Miller units in unchallenged control wells from Miller units recorded upon drug exposure. Mutant variants are shown in purple, wild-type high-expressing $N f_{S} A_{-} E c$ in red, and empty $p U C X$ control in grey. Data are the average of 3 biological repeats \pm SEM (each assay consisting of two technical replicates).

All rescreened variants demonstrated improvements in their ability to induce S33 activation of the SOS response over wild-type NfsA_Ec. The most 
active variants in this assay exhibited a two-fold increase in SOS Miller units over what ultimately turned out to be high-expressing NfsA_Ec (see Section 3.3.1). The NfsA_Ec SDM library had been unknowingly cloned into lowexpressing pUCX, in which enzymes are expressed to a much lower extent (potentially as much as 10-fold lower than enzymes cloned into highexpressing pUCX; see Section 3.3.1.1); therefore their activities in Figure 3-16 were severely underreported relative to a much higher-expressing wildtype NfsA_Ec control.

\subsubsection{IC 50 values generated by lead NfsA_Ec variants with S33}

$\mathrm{IC}_{50}$ values for the lead five NfsA_Ec variants identified in the second-tier screen were determined by screening in SOS-R2 across a dilution series of up to $100 \mu \mathrm{M}$ S33 (Table 3-9). Following the discovery of high- and lowexpressing pUCX, the activities of these variants were compared to a lowexpressing pUCX NfsA_Ec control.

\begin{tabular}{c|c|c|c} 
& \multicolumn{3}{c}{$\begin{array}{c}\text { Fold } \\
\text { reduction } \\
\text { in } \text { IC }_{50} \\
\text { over } \\
\text { NfsA_Ec }\end{array}$} \\
\hline Enzyme & IC $50(\mu \mathrm{M})$ & aa substitutions \\
\hline NfsA SDM 11_9 & $23.0 \pm 0.8$ & 3.8 & R225A/F227S \\
\hline NfsA SDM 15_34 & $25.2 \pm 2.9$ & 3.5 & I5T/R225A/F227S \\
\hline NfsA SDM 11_78 & $25.3 \pm 1.1$ & 3.5 & S41Y/L103M/K222E/R225A \\
\hline NfsA SDM 12_54 & $25.5 \pm 0.8$ & 3.4 & R225A \\
NfsA SDM 1_1 & $25.9 \pm 2.0$ & 3.4 & R225A/F227S \\
\hline Low-expressing & $87.2 \pm 2.2$ & 1.0 & - \\
NfsA_Ec & $>>100$ & & \\
Empty pUCX & & & \\
\hline
\end{tabular}

Table 3-9 IC $I_{50}$ values of S33 mediated growth inhibition of lead variants from SOSR2 NfsA_Ec SDM library challenged with up to $100 \mu$ M S33. S33 mediated growth inhibition was monitored by measuring strain turbidity $\left(O D_{600}\right)$ pre- and postfour hour incubation with a two-fold dilution series of S33, with a top concentration of $100 \mu M$ S33. Percentage growth relative to unchallenged controls was determined and used to calculate the concentration at which 50\% growth inhibition was seen using GraphPad Prism 7.0. Data are the average of 3 biological replicates $\pm S D$. Fold reduction in $I C_{50}$ was calculated in relation to cells containing a low-expressing $N f s A_{-}$Ec plasmid. 
All five variants showed at least a 3.4-fold improvement in sensitising SOSR2 to S33. Two of these variants (11_9 and 1_1) showed identical amino acid substitutions (R225A/F227S), and all five variants contained the R225A substitution, suggesting that it could be a key substitution in improving NfsA_Ec activity with S33.

\subsubsection{Active site modelling for selected NfsA_Ec SDM library mutants}

A

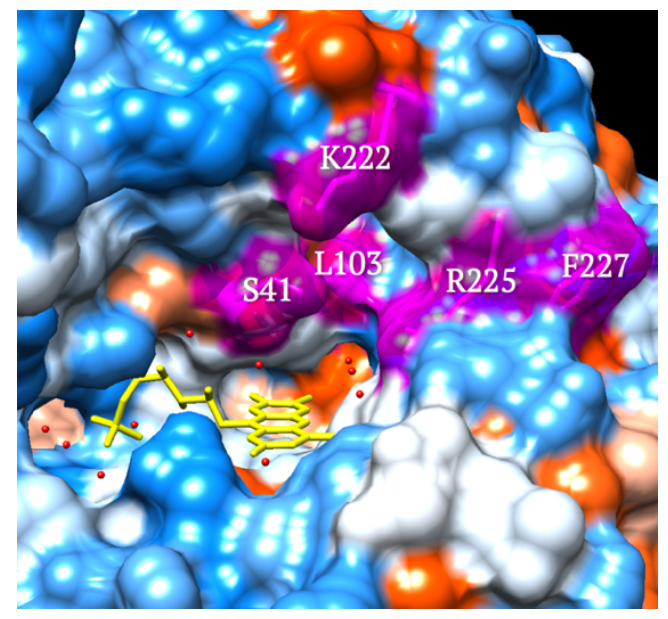

C

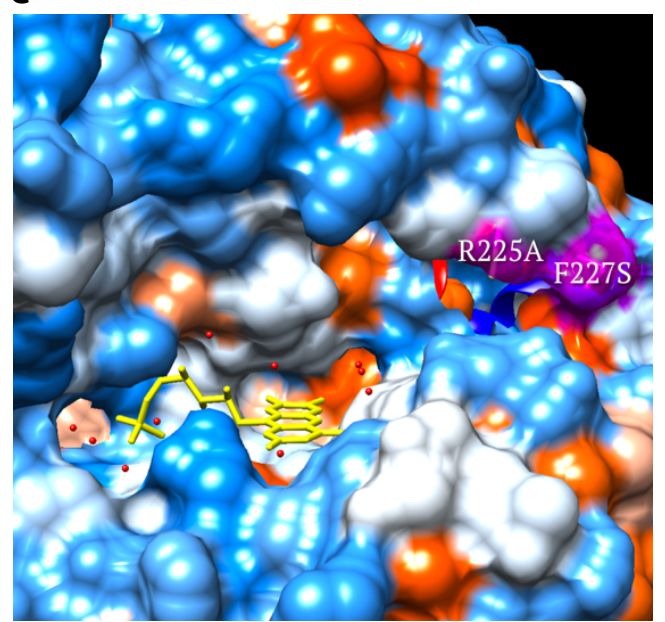

B

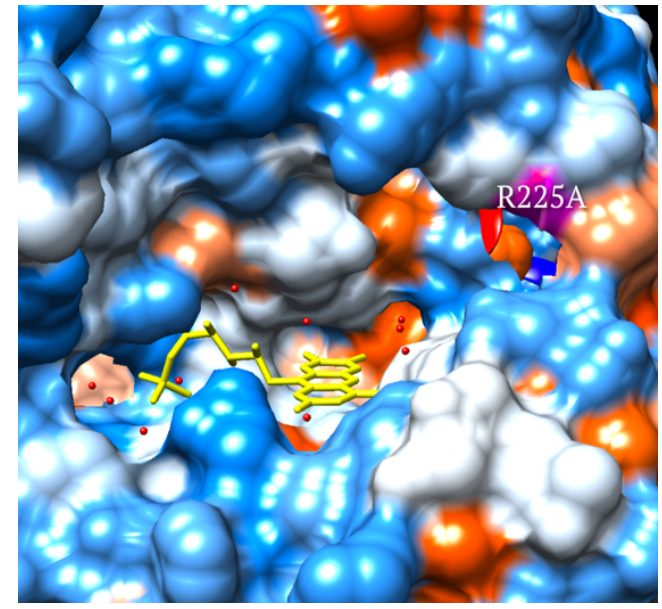

D

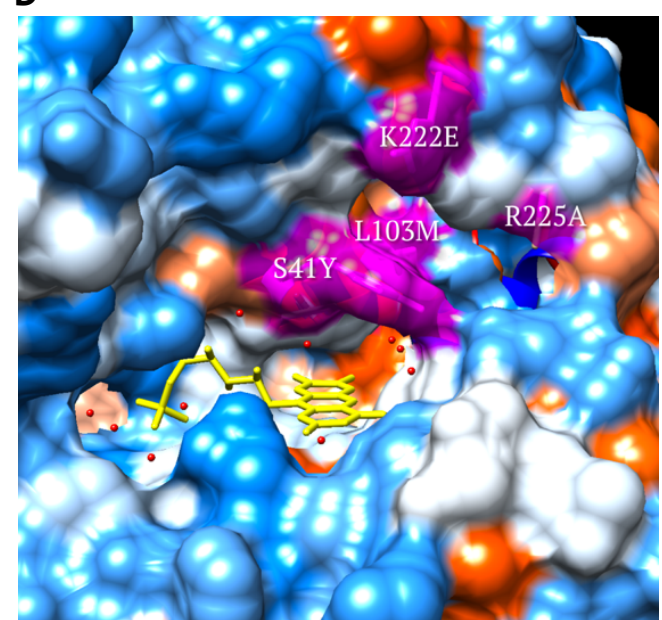

Figure 3-17 Modelling of active site of NfSA_Ec and SDM library variants. A) Modelling of active site residues in NfSA_Ec that are mutated in lead SDM variants, highlighted in purple. The FMN molecule is coloured yellow. B) Modelling of active site of NfSA variant R225A. C) Modelling of active site of $N f_{S} A$ variant R225A/F227S. D) Modelling of active site of $N f_{S} A$ variant $S 41 Y / L 103 M / K 222 E / R 225 A$. 
Modelling of the active site of NfsA_Ec SDM variants (Figure 3-17) show the opening of the right-hand access to the active site upon the conversion of Arg 225 to the much smaller hydrophobic amino acid Ala (Figure 3-17B). A substitution of Val at residue 227 in place of Phe also replaces the parent amino acid with a much small molecule (Figure 3-17C). The additional substitution of Thr at residue 5 in place of Ile does not appear be directly involved in the active site, as this residue occurs at the monomer-monomer interface. The I5T/R225A/F227S triple mutant showed a similar ability as the R225A/F227S to sensitise E. coli to lethal doses of S33 (Table 3-9), suggesting that this additional mutation does not substantially improve enzyme activity with S33.

Finally, the variant S41Y/L103M/K222E/R225A displays quite a different active site to the other three variants, as it involves the substitution of Ser 41 and Leu 103 with larger amino acids (Tyr and Met respectively) in the active site, and of positively charged Lys 222 with negatively charged Glu (Figure 3-17D).

\subsubsection{Reduction of S33 by purified His ${ }_{6}$-tagged purified NfsA_Ec variant proteins}

The four unique variants identified via $\mathrm{IC}_{50}$ assays were cloned into the $\mathrm{His}_{6}$ tag expression vector $\mathrm{pET} 28 \mathrm{a}+$ and purified protein kinetics determined with S33 to test whether activity had also been improved in vitro. The single mutant F227S, isolating an amino acid substitution that appeared in two variants (11_9 and 15_34), was also included (Table 3-10).

All variants exhibited at least 3.5-fold improvement in $k_{\text {cat }} / K_{M}$ with S33 over NfsA_Ec, confirming that these substitutions did improve ability of the enzyme to activate S33. The single R225A substitution lowered the apparent $K_{M}$ by 40 -fold, at the expense of a 3-fold reduction in apparent $k_{\text {cat }}$, with a 14 fold improvement in $k_{\text {cat }} / K_{M}$ overall. The single F227S substitution, on the other hand, retained NfsA_Ec $k_{c a t}$ and lowered the apparent $K_{M}$ by 6-fold, with a 7 -fold improvement in $k_{c a t} / K_{M}$. The combination of these two substitutions in the variant R225A/ F227S did not show a synergistic effect, 
and it only demonstrated a 11 -fold improvement in $k_{c a t} / K_{M}$ over NfsA_Ec. The additional substitution of I5T, which is located at the monomer-monomer interface, in variant I5T/R225A/F227S again worsened activity in vitro, and it only showed a 3.5 -fold improvement in $k_{c a t} / K_{M}$. The variant S41Y/L103M/K222E/R225A, with 4 substitutions in the active site (R225A being the only substitution it shared with other variants) showed a 7 -fold improvement in $k_{\text {cat }} / K_{M}$ Over NfsA_Ec.

In summary, the sole amino acid substitution of R225A generated the greatest improvement in NfsA_Ec activation of S33 in vitro, and additional substitutions in the other three variants only served to worsen apparent $k_{c a t}$ or $K_{M}$ with S33.

\begin{tabular}{|c|c|c|c|c|}
\hline Enzyme & $\boldsymbol{k}_{\text {cat }}\left(\mathbf{s}^{-1}\right)$ & $K_{M}(\mu \mathrm{M})$ & $\begin{array}{l}k_{\text {cat }} / K_{M} \\
\left(\mathbf{s}^{-1} \cdot \mathrm{M}^{-1}\right)\end{array}$ & $\begin{array}{c}\text { NADPH } \\
\text { oxidase } \\
\text { activity } \\
(\mu \mathrm{M} \\
\text { NADPH. } \mu \mathrm{M} \\
\left.\text { enzyme }{ }^{-1} \cdot \mathrm{s}^{-1}\right)\end{array}$ \\
\hline NfsA R225A & $0.69 \pm 0.04$ & $91 \pm 28$ & $7600 \pm 2400$ & $0.35 \pm 0.06$ \\
\hline $\begin{array}{c}\text { NfsA } \\
\text { R225A/F227S }\end{array}$ & $1.3 \pm 0.1$ & $220 \pm 40$ & $5900 \pm 1200$ & $0.23 \pm 0.09$ \\
\hline NfsA F227S & $2.5 \pm 0.2$ & $650 \pm 100$ & $3800 \pm 700$ & $0.51 \pm 0.09$ \\
\hline $\begin{array}{l}\text { NfsA S41Y/L103M } \\
\text { /K222E/R225A }\end{array}$ & $0.16 \pm 0.01$ & $52 \pm 16$ & $3100 \pm 1000$ & $0.16 \pm 0.02$ \\
\hline $\begin{array}{c}\text { NfsA I5T } \\
\text { /R225A/F227S }\end{array}$ & $2.6 \pm 0.4$ & $1400 \pm 400$ & $1900 \pm 600$ & $0.39 \pm 0.02$ \\
\hline NfsA_Ec & $2.0 \pm 0.4$ & $3700 \pm 1100$ & $530 \pm 200$ & $0.22 \pm 0.04$ \\
\hline
\end{tabular}

Table 3-10 Michaelis-Menten kinetic parameters for Nfs__Ec mutants and S33. Initial rates of reduction at varying $S 33$ concentrations and a fixed concentration of excess NADPH (250 $\mu \mathrm{M})$ were monitored at $350 \mathrm{~nm}$, with rates at each $S 33$ concentration measured in at least triplicate. Apparent $K_{M}$ and $k_{\text {cat }}$ at $250 \mu \mathrm{M}$ $N A D P H$ with a glucose oxidase-catalase oxygen scavenging system (12 units.mL ${ }^{1}$ glucose oxidase, 12 units. $\mathrm{mL}^{-1}$ catalase, $50 \mathrm{mM}$ glucose) were calculated using GraphPad 7.0. An extinction coefficient of $9,650 \mathrm{M}^{-1} \mathrm{~cm}^{-1}$ was used and errors represent \pm SEM. NADPH oxidase activity shows enzyme-mediated NADPH oxidation in the absence of a glucose oxidase-catalase oxygen scavenging system, with an extinction coefficient of $4020 \mathrm{M}^{-1} \mathrm{~cm}^{-1}$ used. 


\subsection{Discussion}

The research described in this chapter utilised an existing 58 nitroreductase library, a core resource in the Ackerley lab, for discovery of wild-type nitroreductases exhibiting activity with S33. This was achieved via a combination of in vivo E. coli S33-mediated SOS and growth inhibition assays, and in vitro confirmation of enzyme activity through the measurement of apparent kinetic parameters using His $_{6}$-tagged purified proteins and S33.

\subsubsection{Purified protein kinetics}

A glucose oxidase-catalase oxygen scavenging system was implemented and shown to be able to diminish the background NADPH oxidase activity of purified nitroreductases to allow apparent $K_{M}$ and $k_{\text {cat }}$ parameters to be determined. However, the saturation of S33 at $1.2 \mathrm{mM}$ in the $2 \mathrm{~mm}$ pathlength Uvette meant that only a rough estimation of kinetic parameters could be determined for some nitroreductases possessing a much high apparent $K_{M}$, generating in these cases a large margin of error. To allow for more accurate determination of enzyme reduction of S33 at compound concentrations higher than $1.2 \mathrm{mM}$, measurement through smaller pathlength cuvettes, such as $1 \mathrm{~mm}$ cuvettes could be utilised instead. Furthermore, the continued presence of background NAD(P)H oxidase activity from nitroreductase enzymes was presumably caused by the inability of the glucose oxidase-catalase oxygen scavenging system to scour all of the oxygen from the solution. This background consumption of NADPH impacted the ability to accurately determine initial velocities of S33 reduction by enzymes with low turnover rates $\left(k_{c a t}\right)$ with S33 or with high innate $\mathrm{NAD}(\mathrm{P}) \mathrm{H}$ oxidase activity.

Current efforts are underway in our research group to optimise a NADPH regeneration system, whereby any NADPH oxidised to NADP+ used in the reaction is immediately converted back by glucose dehydrogenase in the presence of D-glucose. This would allow for the sole monitoring of S33 
reduction as no measurement of background $\mathrm{NAD}(\mathrm{P}) \mathrm{H}$ oxidase activity would be required, and would also overcome the need for a glucose oxidasecatalase oxygen scavenging system. The inclusion of purified His $_{6}$-tagged glucose dehydrogenase from $B$. subtilis and D-glucose had been previously shown by the Ackerley group to be able to regenerate reduced NADH cofactor to allow for complete oxidoreductase reduction of $150 \mu \mathrm{M}$ environmental pollutant $\mathrm{Cr}(\mathrm{VI})$ with a limited supply $(50 \mu \mathrm{M})$ of NADH (Robins et al., 2013).

\subsubsection{Evaluation of wild-type candidate nitroreductase enzymes}

The selection of nitroreductase candidates for further evolution work later in this chapter and Chapter 4 revolved both around the natural ability of these enzymes to reduce S33, as well as for their capability to stably express in HCT-116 human cell lines. As discovered in work conducted by previous Ackerley lab researchers, this does not automatically mean that variants derived from a stably-expressed wild-type nitroreductase will also show stable expression in cell lines (Copp et al., 2017; Williams, 2013); however it at least allows for comparison of variants against the wild-type nitroreductase in human cell experiments. Previous research has shown the wild-type candidate enzymes NfsA_Ec, YcnD_Bs and YfkO_Bs to all exhibit stable expression in HCT-116 cell lines (Figure 3-6). These three enzymes all exhibited the ability to sensitise $E$. coli to S33, and activity was confirmed in vitro, albeit with extremely low $k_{c a t} / K_{M}$ ratios (around $10^{2} \mathrm{~s}^{-1} \mathrm{M}^{-1}$ ) suggesting inaccuracies in calculations of S33 extinction coefficients. Nevertheless they give us benchmarks to judge the success of attempts at nitroreductase evolution.

\subsubsection{Screening of site-targeted nitroreductase libraries}

Two different targeted mutagenesis libraries of the nitroreductases NfsA_Ec and YcnD_Bs, which had been previously created and screened for PR-104A 
activity, were utilised as a resource for rapid screening of variants with improved S33-activating capabilities. Screening of these libraries was conducted through the monitoring the effects of activated S33 on the endogenous E. coli SOS response via a lacZ reporter gene.

Screening of YcnD_Bs libraries yielded three variants with improved activity with S33 in vivo, however in vitro two of these variants exhibited a worse $k_{\text {cat }} / K_{M}$ than YcnD_Bs. Alternatively their additional reduced NADPH oxidase activity could indicate changes in the active site architecture that may also have diminished the catalytic activity of these variants with other substrates present in the E. coli cytoplasm. This could in turn diminish competitive inhibition with S33 and lead to a greater in vivo activity, even with a lower $k_{\text {cat }} / K_{M}$ with S33 than wild type YcnD_Bs. A similar phenomenon was recently described by our group in the development of a variant of NfsA_Ec that was several-fold more effective at activating PR-104A within E. coli cells, despite having a lower $k_{c a t} / K_{M}$ with PR-104A in vitro (Copp et al., 2017). The third YcnD variant yielded from screening, 227_60, showed a 3-fold improvement in $k_{\text {cat }} / K_{M}$ over YcnD_Bs.

Four NfsA_Ec variants exhibited substantially improved in vivo activity in $E$. coli that translated into improvements in purified protein kinetics in vitro, with up to a 14 -fold increase in $k_{\text {cat }} / K_{M}$ over NfsA_Ec generated by a single R225A substitution. Structural modelling of this variant suggested improvements in activity could have been due to the amino acid substitution allowing for a more open active site. Unlike the YcnD variants, NfsA variants did not exhibit reduced NADPH oxidase activity.

\subsubsection{High vs low-expressing pUCX plasmids}

Finally, the discovery of the existence of both low-expressing and highexpressing pUCX plasmids confounded attempts to accurately confirm the activity of improved variants in vivo both in this chapter and in Chapter 4 . This does raise the possibility that variants investigated by other members of the Ackerley group may have had their activity under-reported, if cloned 
into low-expressing pUCX and compared to a high-expressing control. Moreover, this study offers an explanation for why the pUCX:KG plasmid developed by Dr Prosser exhibits lower levels of protein expression than the pUCX plasmid from which it was derived (Prosser et al., 2015); it transpires that pUCX:KG was constructed from low-expressing pUCX.

\subsubsection{Alternative methods for screening nitroreductase variant libraries}

We have shown that improved nitroreductase variants could be recovered from previously constructed site-targeted mutant libraries already present in the Ackerley lab, through the use of a lacZ-based SOS assay to screen for enzyme activation of S33. Parallel efforts to engineer S33 specificity in the nitroreductases YcnD_Bs and YfkO_Bs through the creation of novel randomly-mutated libraries using error-prone PCR, and screening for improved variants using the GFP SOS assay and fluorescence-activated cell sorting (FACS) are described in Chapter 4. 


\section{Chapter 4. Directed evolution of YcnD_Bs and YfkO_Bs for improved S33 activation via random mutagenesis}

\subsection{Introduction}

Chapter 3 describes how variants were successfully recovered from sitetargeted libraries of the NfsA enzymes NfsA_Ec and (to some extent) YcnD_Bs that demonstrated improved capabilities to activate the 5nitroimidazole PET imaging probe S33. As NfsB family enzymes often exhibit different kinetic profiles to NfsA family enzymes (i.e., tend to exhibit substantially higher $k_{c a t}$ and $K_{M}$ for a given substrate, as discussed in Section 1.3), there was also interest in evolving an NfsB enzyme for improved activation of S33, in case the different kinetic profile might in practice translate to superior activity in vivo. Furthermore, our collaborators in an ongoing multi-centre project seeking to take nitroreductase-armed Clostridium sporogenes to human GDEPT clinical trials had empirical evidence that NfsB-family nitroreductases are generally expressed at higher levels in C. sporogenes than NfsA-family nitroreductases, and hence we were interested in evolved NfsB-family enzymes (Dr David Ackerley, personal communication).

Interrogation of candidates from the 58 nitroreductase library described in Chapter 3 showed that the E. coli NfsB orthologue YfkO_Bs (from B. subtilis) exhibited the ability to reduce S33 both in vivo in E. coli and in vitro, and was also capable of stable expression in cultured human cells. However, no solved crystal structure for YfkO_Bs or any closely related relatives has yet been described, with the closest being that for FrxA from Helicobacter pylori (RCSB Protein Data Bank ID:2H0U), which only exhibits 31\% identity with YfkO_Bs. Without the ability to confidently select active site residues for creation of site-targeted libraries, random mutagenesis libraries for YfkO_Bs were instead created using error-prone PCR (epPCR) and screened via GFP SOS assay in an attempt to identify improved S33-activating variants. 
Simultaneously, epPCR mutagenised libraries for YcnD_Bs were also created and screened for variants that exhibited higher activity with S33 than the lead variants reported in Chapter 3. Chronologically, the work described in this chapter was conducted in parallel with much of that described in

Chapter 3.

\subsubsection{Directed evolution of enzymes via random mutagenesis}

Directed evolution most frequently involves successive rounds of introduction of mutations into the gene encoding a protein, followed by screening for, or selection of, variants that exhibit improved enzyme activity with a target substrate. In each round, variants that exhibit better activity than their predecessors are selected for subsequent rounds of evolution, in a manner often described as climbing a peak of protein 'fitness' towards a local maximum i.e. the most active attainable variant (Packer and Liu, 2015; Romero and Arnold, 2009). As seen in Chapter 3, enzyme variants can be generated through the selective targeting of active site residues predicted to be important for activity with that particular substrate; however, this method requires structural and mechanistic knowledge of the enzyme. Random mutagenesis, where mutations are introduced indiscriminately into the gene encoding the enzyme, can be conducted in the absence of this knowledge; furthermore, mutations far away from the active site have frequently been shown to improve enzyme activity (Oue et al., 1999; Shimotohno et al., 2001; Spiller et al., 1999). Random mutagenesis libraries are typically generated through epPCR, where random base substitutions are introduced into the gene through the use of low-fidelity DNA polymerases. However, one of the disadvantages of randomly introduced mutations is their high propensity to substantially reduce or completely obliterate enzyme activity. A review by Romero and Arnold (2009) describes randomly generated single amino acid substitutions as likely to only be beneficial $0.01-1 \%$ of the time, while up to $50 \%$ of substitutions can be strongly deleterious (Romero and Arnold, 2009). Therefore, the generation of large 
error-prone libraries (e.g. $>10^{6}$ unique variants) must be paired with highthroughput screens to allow for the effective discovery of improved variants. The previously described LacZ-based SOS assay in Section 3.1.3 can only screen libraries containing $\sim 10^{3}$ unique variants 96 -well microplates in a reasonable timeframe; however FACS-based enzyme screens, where $>10^{4}$ events can be sorted per second, offer promise for high-throughput screening of epPCR libraries.

\subsubsection{GFP SOS assay and fluorescence-activated cell sorting}

As shown in Figure 3-11, the GFP-based SOS assay can be used to screen for active nitroreductases by monitoring levels of GFP production from E. coli SOS-R4 cells bearing active nitroreductase variants. Cells over-expressing variants that could reduce S33 generated higher levels of GFP than those containing inactive nitroreductases when challenged with sub-lethal concentrations of the PET imaging probe, due to the ability of activated S33 to induce the endogenous SOS response and promote GFP production. The Ackerley research group has also shown that the GFP SOS assay can be utilised for ultra high-throughput selection of active variants using a flow cytometer (Copp et al., 2014). In a manner similar to the plate reader-based detection of GFP produced by cells following challenge with a prodrug or probe, the fluorescence of challenged $E$. coli SOS-R4 cells can be visualised in a flow cytometer following excitation with a $488 \mathrm{~nm}$ blue laser. Individual highly fluorescent cells (bearing more active nitroreductase variants) can then be sorted into a tube or 384-well microplate containing recovery media (Figure 4-1). 
1) SOS-R4 cells overexpressing nitroreductase variants are challenged with S33. Variants that are more active with $\mathrm{S} 33$ induce more GFP production.
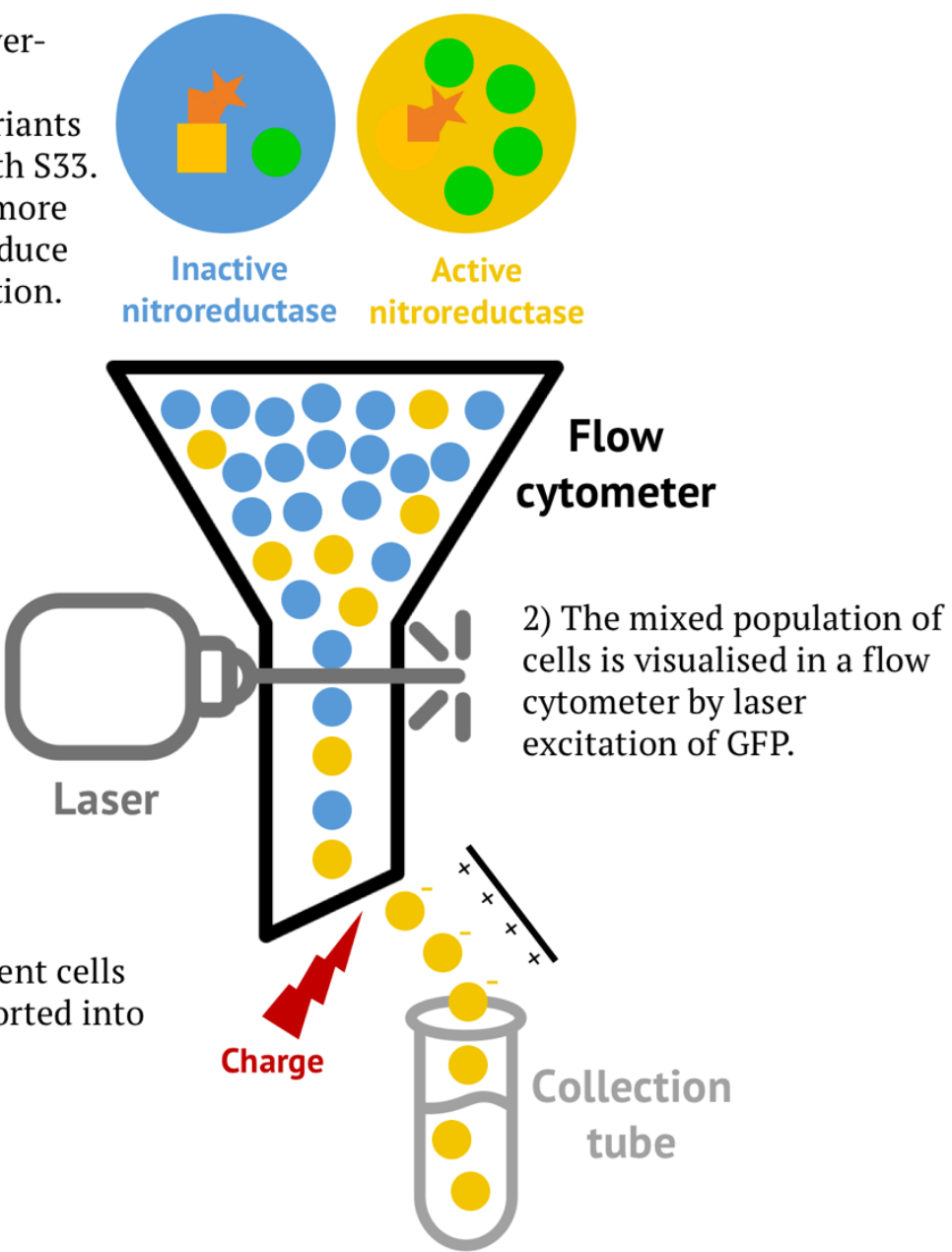

Figure 4-1 Schematic showing fluorescence-activated cell sorting (FACS) of E. coli SOS-R4 cells over-expressing inactive or active nitroreductases.

To demonstrate successful FACS-based selection of active nitroreductases, the prodrug CB1954 was used to challenge mixed populations of $E$. coli SOSR4 cells over-expressing either a nitroreductase active with CB1954, YcnD_Bs, or the putative nitroreductase YdjA_Ec (Choi et al., 2008) which exhibits no activity with CB1954 (Prosser et al., 2013). YcnD_Bs-expressing cells comprising $0.1 \%$ of a mixed population were successfully enriched 380 fold (to 38\% of the population) following a single FACS sort (Copp et al., 2014). 


\subsubsection{Previous FACS selection of a YfkO_Bs library}

A pilot study during my Honours research had attempted to use FACS to select for improved S33-activating YfkO_Bs variants from a small random mutagenesis library ( $4 \times 10^{3}$ unique variants). However, downstream screening of FACS-selected variants using the GFP SOS assay showed most variants exhibited only limited improvements in activity over wild-type YfkO_Bs; furthermore up to a quarter of variants exhibited little to no activity with S33 (Rich, 2012). It was postulated that the latter variants may have produced higher levels of GFP in response to stress from the flow cytometry sorting, leading to their selection alongside variants that did exhibit activity with S33. In addition, variants selected within a gate that demarked only the top $5 \%$ of fluorescent events showed extremely low recovery rates (<10\% of sorted cells; Rich, 2012), possibly due to a high proportion of these cells having accumulated irreversible DNA damage from exposure to activated S33 as well as stress from the cell sorting process. As both these observations suggested that FACS sorting might prove problematic for recovering the top variants from a library, the current chapter describes an investigation into an additional method that might allow for the removal of generally inactive nitroreductases from random mutagenesis libraries, and thereby increase the selection of more active variants.

\subsubsection{Niclosamide}

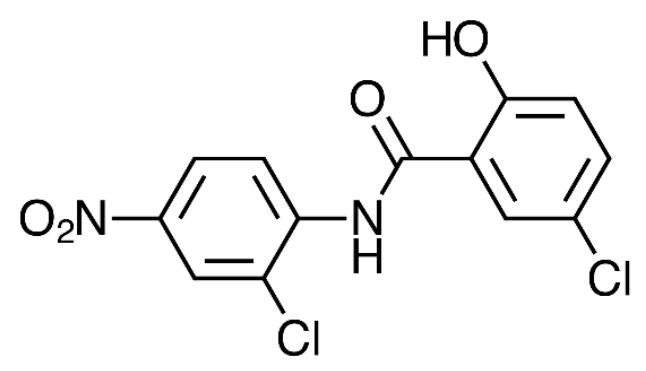

Figure 4-2 Chemical structure of niclosamide. 
This method involved use of the nitroaromatic drug niclosamide [N-(2'chloro-4'-nitrophenyl)-5-chlorosalicylamide] (chemical structure shown in Figure 4-2), which is used clinically in the treatment of intestinal parasitic worms, its mode of action occurring through both the uncoupling of oxidative phosphorylation and stimulation of mitochondrial adenosine triphosphatase (Al-Hadiya, 2005; Ditzel and Schwartz, 1967). Cancer researchers have also shown that it exhibits anti-cancer properties both in vitro and in vivo, with the ability to inhibit multiple signalling pathways upregulated in tumour cells, and it also targets mitochondria to stimulate cellular apoptosis (Li et al., 2014).

Niclosamide has also been shown to exhibit toxic effects on Gram-positive bacteria including Staphylococcus, Enterococcus and Clostridia species (Gooyit and Janda, 2016; Rajamuthiah et al., 2015), and its suggested mechanism of action against Clostridium involves the stimulation of membrane depolarisation (Gooyit and Janda, 2016). In Gram-negative bacteria it has been shown to inhibit quorum sensing in Pseudomonas aeruginosa (Imperi et al., 2013), and metabolites generated by nitroreduction of niclosamide have been implicated in its activity against Salmonella (Beristain-Castillo et al., 2013; Espinosa-Aguirre et al., 1991). Previous work conducted by our research group has also found niclosamide to act as an 'anti-prodrug' - that is, it is selectively toxic to E. coli cells lacking the drug efflux pump TolC (Figure 4-3); however over-expression of some nitroreductases also had a protective effect against cell death (Figure 4-4; Ackerley and Copp, 2016).

Furthermore, the Ackerley lab had also shown that variants from a heavily randomised active site codon library of NfsA_Ec (the 7SM library; discussed further in Chapter 5) that were capable of growth on LB agar supplemented with $0.5 \mu \mathrm{M}$ niclosamide exhibited far greater activity with the 5nitroimidazole prodrug metronidazole than randomly picked variants that had not been pre-selected using niclosamide (Little, 2015). We were therefore interested in the potential for niclosamide to eliminate inactive 
variants from random mutagenesis libraries prior to screening in directed evolution studies.

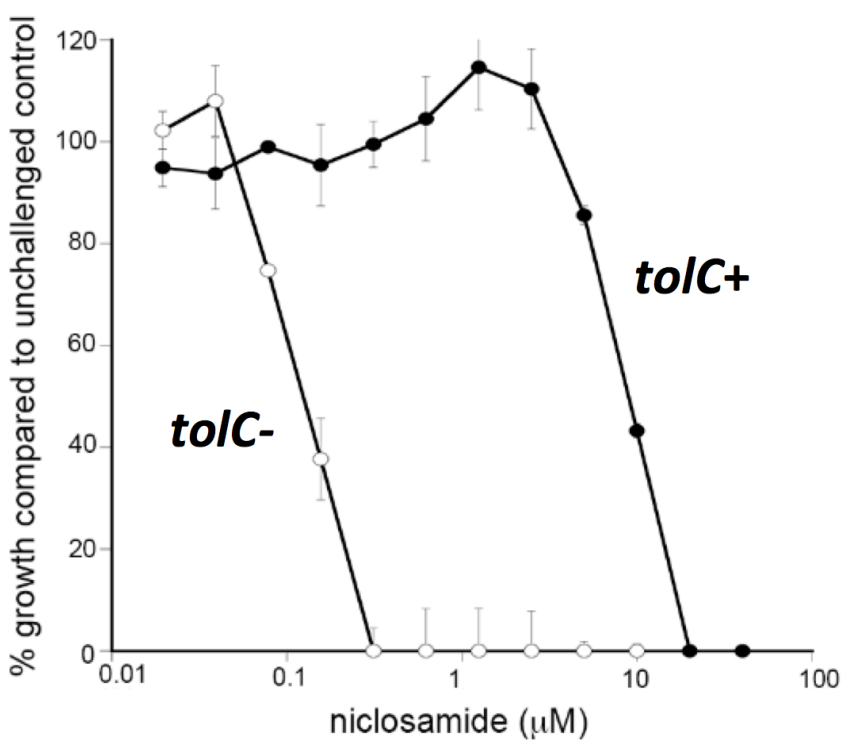

Figure 4-3 Growth inhibition of isogenic tolC- and tolC+ E. coli strains in response to increasing niclosamide challenge. Niclosamide-dependent growth inhibition was monitored by measuring strain turbidity $\left(\mathrm{OD}_{600}\right)$ pre- and post-four hour incubation with a two-fold dilution series of niclosamide, with a top concentration of $40 \mu \mathrm{M}$ niclosamide. Percentage growth relative to unchallenged controls was determined and plotted on the y-axis. Data are the mean of independent triplicate repeats and errors $\pm 1 S D$. Figure reproduced from Ackerley and Copp, (2016) with permission from Dr David Ackerley. 


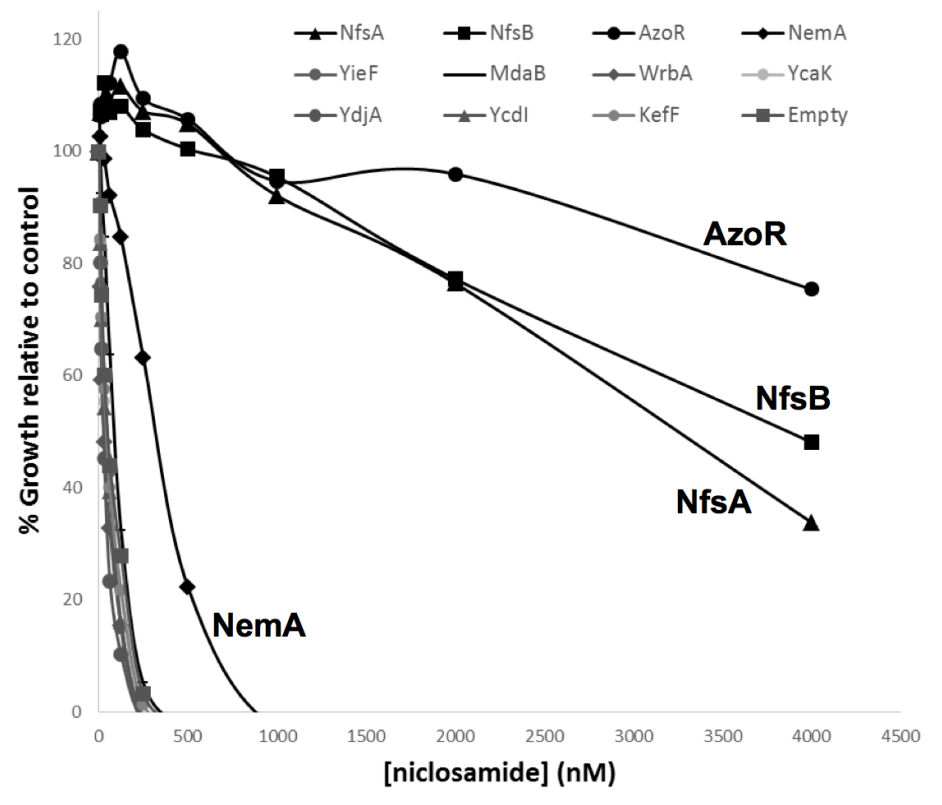

Figure 4-4 Growth inhibition of tolC- E. coli strain bearing pUCX-expressing nitroreductases in response to increasing niclosamide challenge.Niclosamidedependent growth inhibition was monitored by measuring strain turbidity $\left(O D_{600}\right)$ pre- and post-four hour incubation with a two-fold dilution series of niclosamide, with a top concentration of $4 \mu M$ niclosamide. The particular overexpressed nitroreductase (or empty plasmid) present in each strain is indicated in the figure legend. Percentage growth relative to unchallenged controls was determined and plotted on the y-axis. Data are the mean of independent triplicate repeats and errors \pm 1 SD. Figure reproduced from Ackerley and Copp, (2016) with permission from Dr David Ackerley.

\subsection{Aims}

- Evaluate the potential for niclosamide for use as a positive selection agent for active nitroreductase variants.

- Generate error-prone libraries of YcnD_Bs and YfkO_Bs, followed by FACS to recover improved S33-activating variants.

- Use the most active variants identified in each round of mutagenesis and screening as gene templates for further rounds of S33 directed evolution.

Originally multiple rounds of directed evolution were planned for both YcnD_Bs and YfkO_Bs; however difficulties encountered with the E. coli SOSR4 GFP reporter strain and generation of evolved YcnD_Bs variants limited 
the work conducted in this chapter to only two rounds of random mutagenesis and identification of improved variants.

\section{$4.3 \quad \underline{\text { Results }}$}

\subsubsection{Evaluation of nitroreductase positive selection compounds}

To evaluate the efficacy of niclosamide as a selection compound for generally active nitroreductases, E. coli 7NT cells (which lack the tolC gene) over-expressing variants from the 58 nitroreductase library were incubated with $0.5 \mu \mathrm{M}$ niclosamide (Figure 4-5). Similar to previously observed effects (Figure 4-4), cells bearing an empty pUCX plasmid or generally inactive nitroreductase variants showed almost complete growth inhibition in the presence of niclosamide, whereas members of the NfsA, NfsB and AzoR nitroreductase families could protect against cell death (Figure 4-5). Complete growth inhibition of SOS-R4 cells bearing the empty pUCX plasmid was also observed when plated on LB agar plates containing $0.5 \mu \mathrm{M}$ niclosamide, whereas cells over-expressing YcnD_Bs or YfkO_Bs showed no impairment in their ability to form colonies (results not shown). This suggested that inactive variants from $y c n D \_B s$ or $y f k O_{-} B s$ random mutagenesis libraries could simply be removed in a one-step fashion by the plating of SOS-R4 cells expressing these libraries on niclosamide-containing agar plates. 


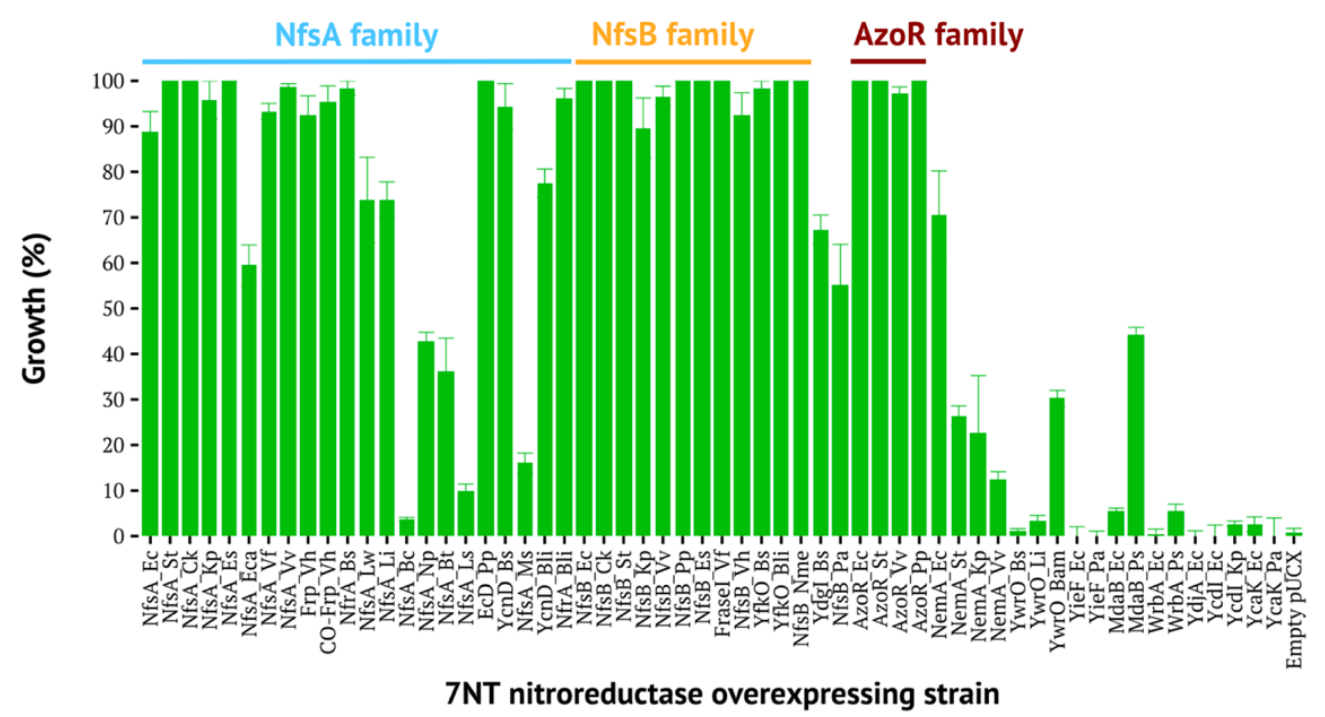

Figure 4-5 Growth of 7NT nitroreductase over-expression strains, post-challenge with $0.5 \mu \mathrm{M}$ niclosamide. Growth of strains resistant to niclosamide was monitored by measuring culture turbidity $\left(O D_{600}\right)$ pre- and post-four hour incubation with $0.5 \mu M$ niclosamide. Percentage growth relative to unchallenged controls was determined. Data are the average of 3 biological replicates \pm SEM.

\subsubsection{YcnD_Bs and YfkO_Bs Round 1 error-prone library generation and screening}

\subsubsection{Creation of libraries and effects of niclosamide selection}

For the first round of directed evolution of YcnD_Bs and YfkO_Bs enzymes to improve their S33-activating capabilities, epPCR products were generated using Mutazyme II error-prone polymerase from wild-type ycnd_Bs and $y f k O_{-} B s$ templates, respectively. The amplified genes were cloned into highexpressing pUCX (as it turned out, although the existence of two different forms of pUCX - Section 3.3.1 - had not yet been established) and transformed into the E. coli SOS-R4 reporter strain. Sequence analysis of ten random variants from each library confirmed an average of two base-pair mutations (generally resulting in two amino acid substitutions) had been introduced into each variant. The overall library sizes were relatively small, with only 8000 variants in the YcnD_Bs library and 18,000 variants in the YfkO_Bs library; however, as this first round was viewed largely as a proof- 
of-concept study and with time constraints due to pre-existing flow cytometry bookings, the decision was made to continue with these libraries.

These libraries were plated on LB agar plates containing $0.5 \mu \mathrm{M}$ niclosamide. A $0.5 \mu \mathrm{M}$ concentration of niclosamide was found to select for $30 \%$ of the YcnD_Bs variant library (designated the YcnD M1 library) and 10\% of the YfkO_Bs variant library (designated the YfkO M1 library), with the final sizes of the niclosamide-selected libraries being 2400 and 1800 variants respectively.

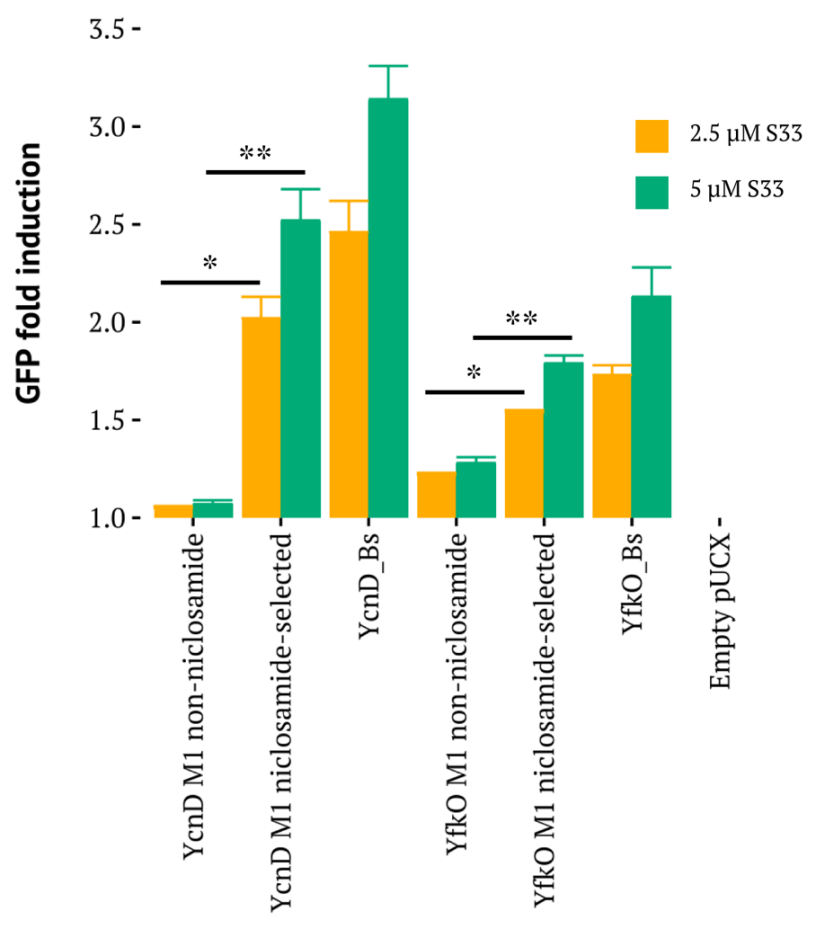

SOS-R4 nitroreductase over-expressing strain

Figure 4-6 S33 mediated induction of fluorescence in E. coli SOS-R4 strains overexpressing either native YfkO, native YcnD, or the YfkO or YcnD M1 libraries pre- or post- selection with $0.5 \mu \mathrm{M}$ niclosamide, challenged with $2.5 \mu \mathrm{M}$ or $5 \mu \mathrm{M}$ S33. Over-expression strains were grown in individual wells of 96-well microplates and challenged with $2.5 \mu M$ (yellow bars) or $5 \mu M S 33$ (green bars) for four hours prior to quantification of the SOS response by measurement of GFP fluorescence. Fold induction was derived from the fluorescence recorded on drug exposure divided by that of unchallenged control wells. Data are the average of 3 biological replicates \pm SEM. Differences in fluorescence between non-selected and niclosamide-selected ycnD_Bs or yfkO_Bs M1 error-prone libraries were statistically significant when compared using unpaired Student's t-tests $-*=p$ value $<0.05, * *=p$ value $<0.002$. 
The comparison of the GFP SOS responses in the pooled variant libraries pre- and post-niclosamide selection showed a significant increase in SOSinduced GFP response to S33 in both the YcnD_Bs and YfkO_Bs libraries (up to 2.3-fold and 1.4-fold increases respectively) following niclosamide selection (Figure 4-6). This suggested niclosamide selection led to the successful enrichment for variants that were more active with S33. However, the average fluorescence of both the YcnD M1 and YfkO M1 libraries was lower than that observed for wild-type YcnD_Bs and YfkO_Bs respectively, indicating that a majority of variants in the library still exhibited lower activity with S33 than the parent enzyme.

\subsubsection{Fluorescence-activated cell sorting (FACS) of the YcnD_Bs and YfkO_Bs M1 libraries}

For flow cytometry selection of the most active M1 library variants, subcultures of the YcnD_Bs and YfkO_Bs M1 libraries were challenged with $5 \mu \mathrm{M}$ S33. Upon profiling of the fluorescent cells in the flow cytometer, careful consideration was given to the gates chosen for selection of the most fluorescent variants (see P1 gates in Figure 4-7). For the YfkO M1 library a gate was selected that included the top $10 \%$ of events occurring in wild-type YfkO_Bs (which encompassed 16\% of fluorescent events occurring in the YfkO M1 library; Figure 4-7B). A similar gating strategy for the YcnD M1 library was not possible as the gate demarking the top $10 \%$ of fluorescent events occurring in wild-type YcnD_Bs only allowed $<5 \%$ of YcnD M1 library events to be selected. The gate was therefore expanded out to include the top 30\% of fluorescent events occurring in wild-type YcnD_Bs (Figure 4-7A) to allow for the selection of a higher proportion of recoverable variants.

The cells giving rise to these events were sorted into individual wells of 384well microplates containing recovery media, with 768 individual YcnD M1 events and 1152 individual YfkO M1 events collected and recovered. Recovery rates for sorted variants for each library were approximately $13 \%$, and all recovered variants were picked into 96-well plates for further 
downstream analysis. In total two 96-well plates for YcnD M1 variants and three 96-well plates for YfkO M1 variants were picked, with duplicate wildtype parent enzymes and one empty pUCX control in SOS-R4 cells included on each plate.
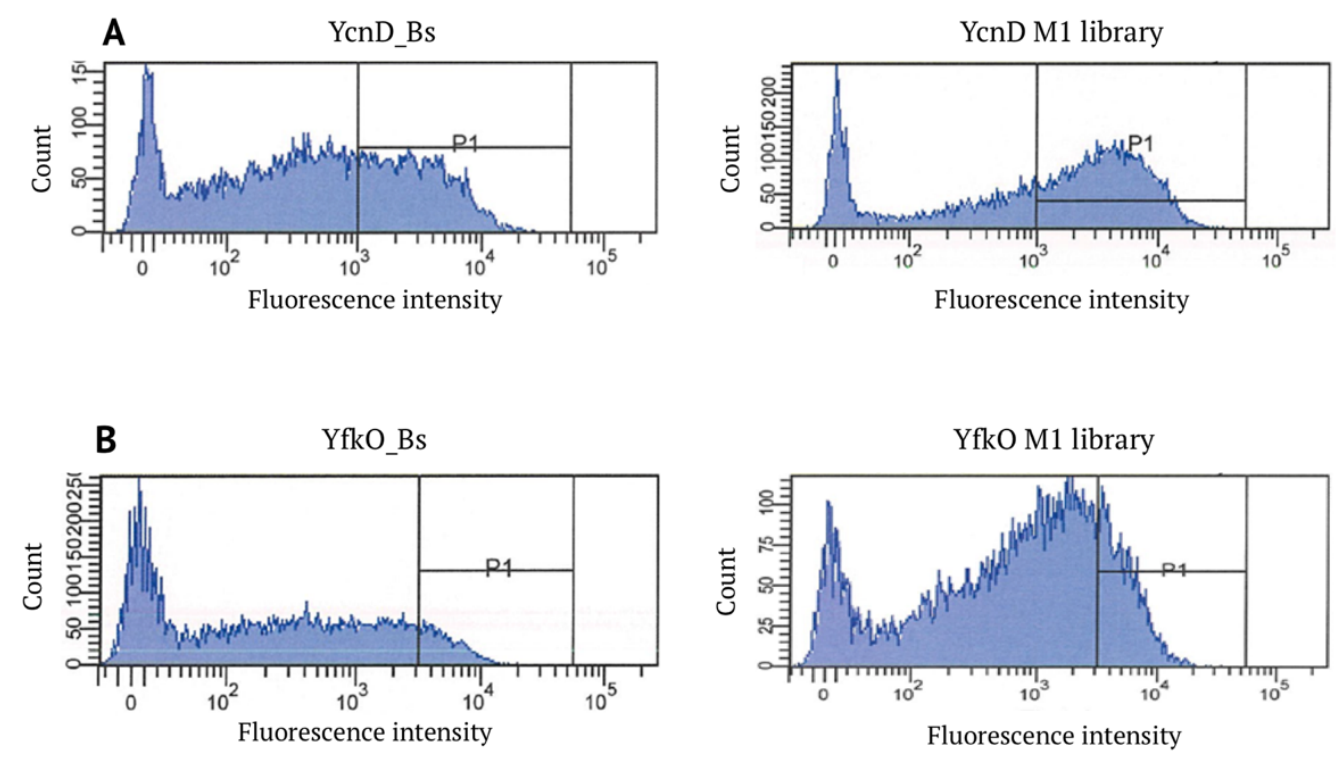

Figure 4-7 Fluorescence of individual cells of wild-type YcnD_Bs and YfkO_Bs, and YcnD M1 and YfkO M1 libraries in SOS-R4 as observed in a FACS Vantage SE-Diva flow cytometer, following challenge with S33. SOS-R4 cells over-expressing nitroreductase variants were challenged with $5 \mu \mathrm{M} S 33$ for $4 \mathrm{~h}$, after which they were excited by $488 \mathrm{~nm}$ light and fluorescence was detected with a 530/30 nm band pass filter. Individual cell counts are shown on the y-axis, and fluorescence intensity observed in cells are shown on the $x$-axis. The P1 gate used for selection of the most fluorescent YcnD M1 cells was gated on the top 30\% fluorescent events occurring in wild-type YcnD_Bs. The P1 gate used for selection of the most fluorescent YfkO M1 cells was gated on the top 9\% of fluorescent events occurring in wild-type $Y \mathrm{FkO}_{-} B \mathrm{~s}$. The high left-hand peak in all plots is noise deriving from the M9 minimal media E. coli cells were suspended in (media-only control not shown).

\subsubsection{Screening of FACS-selected YcnD and YfkO M1 libraries using the GFP SOS assay}

Characterisation of FACS-selected YcnD and YfkO M1 variants for improvements in S33-activating capabilities was conducted using GFP SOS assays, with cells challenged using $2.5 \mu \mathrm{M}$ S33. Each recovered plate of 
variants was screened in triplicate (three biological replicates), with GFP output measured and reported as fold increase in fluorescence over unchallenged cells (Figures 4-8 and 4-9). The most active variants from the sorted YcnD M1 library induced a >1.4-fold increase in fluorescence over wild-type YcnD_Bs, and the most active variants from the sorted YfkO M2 library induced a >1.7-fold increase in fluorescence over wild-type YfkO_Bs. Furthermore, all variants exhibited activity with S33, indicating that the combination of niclosamide pre-selection and FACS allowed for the selection of exclusively S33-active variants.

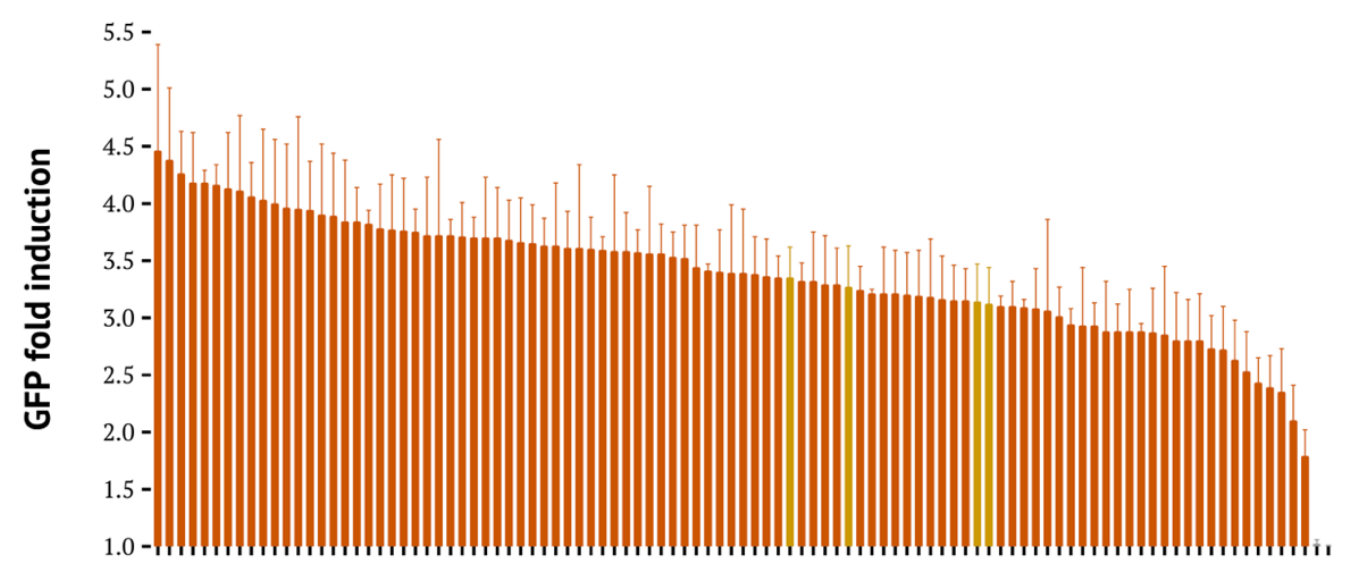

SOS-R4 nitroreductase overexpressing strain

Figure 4-8 S33 mediated induction of fluorescence in E. coli SOS-R4 strains overexpressing either native YcnD, empty $p U C X$ or YcnD M1 libraries post-FACS, challenged with $2.5 \mu \mathrm{M}$ S33. Over-expression strains were grown in 96-well microplates and challenged with $2.5 \mu M S 33$ for four hours prior to quantification of SOS response by measurement of GFP fluorescence. Fold induction is derived from the fluorescence recorded on drug exposure divided by that of unchallenged control wells. Data are the average of 3 biological replicates \pm SEM. Four wild-type YcnD_Bs controls (yellow) and two empty $p U C X$ controls (grey, far right) were included, with YcnD_Bs M1 library variants shown in orange. 


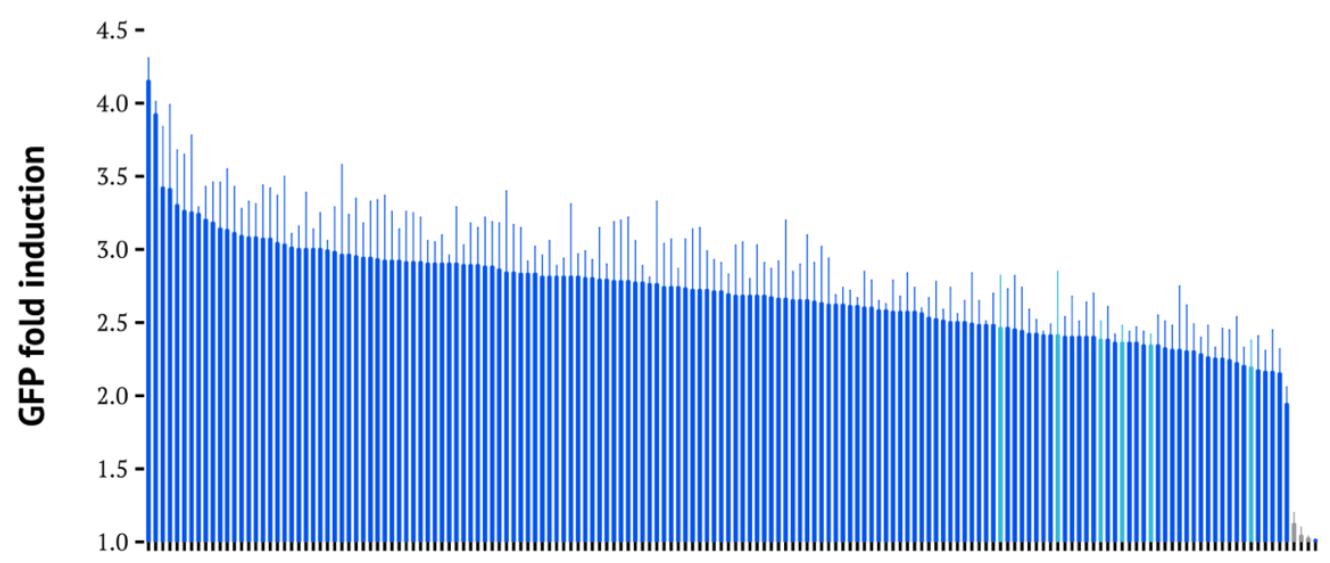

SOS-R4 nitroreductase overexpressing strain

Figure 4-9 S33 mediated induction of fluorescence in E. coli SOS-R4 strains overexpressing either native YfkO, empty pUCX, or the YfkO M1 library post FACS, challenged with $2.5 \mu \mathrm{M}$ S33. Over-expression strains were grown in 96-well microplates and challenged with $2.5 \mu M S 33$ for four hours prior to quantification of $S O S$ response by measurement of GFP fluorescence. Fold induction is derived from the fluorescence recorded on drug exposure divided by that of unchallenged control wells. Data are the average of 3 biological replicates \pm SEM. Six wild-type YfkO_Bs controls (aqua) and three empty $p U C X$ controls (grey, far right) were included, with YfkO M1 library variants shown in blue.

\subsubsection{Growth inhibition (IC $C_{50}$ ) assays with YcnD and YfkO M1 libraries and S33}

The most active four variants from each of the YcnD and YfkO M1 libraries as determined by GFP SOS screens were then subjected to further assessment of S33-reducing activity via more sensitive growth inhibition (IC 50$)$ assays.

These assays were initially conducted in the SOS-R4 strain; however it was later discovered that the pANODuet-1:GFP plasmid contributed to the sensitising of SOS-R4 cells to lethal concentrations of S33 (see Section 4.3.4). In the case of these $\mathrm{IC}_{50}$ assays conducted in the SOS-R4 strain, variants appeared to demonstrate up to a 4 -fold reductions in $\mathrm{IC}_{50}$ over wildtype parent enzymes (results not shown). Furthermore, it was later found that the top $y c n D$ variants had all been cloned into high-expressing pUCX and these variants had been compared to a low-expressing YcnD_Bs control (see Section 3.3.1). However, as these issues were not apparent until Section 4.3.3.3, initial results indicated that the activities of all lead YcnD 
and YfkO M1 variants with S33 were substantially higher than parent enzymes (results not shown).

In Section 4.3.3.3, $\mathrm{IC}_{50}$ assays were conducted in $E$. coli $7 \mathrm{NT}$ cells (not containing the pANODuet-1:GFP plasmid) over-expressing these lead variants across a dilution series of up to $70 \mu \mathrm{M}$ S33 (Tables 4-2 and 4-4), and the activities reported as fold-reductions in $\mathrm{IC}_{50}$ values over the wildtype high-expressing parent enzyme (results for YfkO M1 and M2 variants duplicated here in Tables 4-1 and 4-2). Of the sorted YcnD M1 variants, it became apparent that no significantly improved variants were found after this first round of directed evolution. However, all four leading YfkO M1 variants demonstrated at least a 1.6-fold improvement in sensitising 7NT cells to S33 over wild-type YfkO_Bs, with the lead variant M1 3_44 showing a 2.1-fold improvement in activity. Each of the four YfkO M1 variants showed merely a single amino acid substitution from wild-type YfkO_Bs.

\section{Fold \\ reduction in \\ $\mathrm{IC}_{50}$ over}

WT

\begin{tabular}{c|c|c|c} 
Enzyme & \multicolumn{1}{c}{$\mathbf{I C}_{50}(\boldsymbol{\mu M})$} & YcnD_Bs & aa substitutions \\
\hline YcnD_M1 1_19 & $15.3 \pm 2.8$ & 1.2 & N141D/Q178R/Q222H \\
YcnD_M1 1_22 & $15.5 \pm 1.8$ & 1.2 & G223D \\
YcnD_M1 1_18 & $16.9 \pm 0.8$ & 1.1 & Q32R/E147G \\
YcnD_M1 1_31 & $16.9 \pm 0.6$ & 1.1 & L128F \\
High-expressing & $18.5 \pm 1.6$ & 1.0 & - \\
YcnD_Bs & & - & \\
Empty pUCX & $>>70$ & - &
\end{tabular}

Table 4-1 I $C_{50}$ values of $S 33$ mediated growth inhibition of YcnD M1 variants. S33dependent growth inhibition in 7NT was monitored by measuring strain turbidity $\left(\mathrm{OD}_{600}\right)$ pre- and post-four hour incubation with a two-fold dilution series of S33, with a top concentration of $35 \mu M$ S33 to conserve drug stocks. Percentage growth relative to unchallenged controls was determined and used to calculate the concentration at which 50\% growth inhibition was seen using GraphPad Prism 7.0. Data are the average of 3 biological replicates $\pm S D$. 


\begin{tabular}{c|c|c|c} 
Enzyme & \multicolumn{4}{c}{$\begin{array}{c}\text { Fold } \\
\text { reduction in } \\
\text { IC } \text { I }_{50} \text { over WT } \\
\text { YfkO_Bs }\end{array}$} & aa substitutions \\
\hline YfkO_M1 3_44 & $22.5 \pm 4.4$ & 2.1 & T122R \\
\hline YfkO_M1 1_10 & $27.5 \pm 3.4$ & 1.7 & F82L \\
YfkO_M1 3_2 & $28.2 \pm 3.9$ & 1.7 & M110I \\
YfkO_M1 3_20 & $29.2 \pm 4.8$ & 1.6 & L133H \\
\hline WT YfkO_Bs & $47.1 \pm 6.9$ & 1.0 & \\
\hline Empty pUCX & $>>70$ & - & \\
\hline
\end{tabular}

Table 4-2 IC 50 values of S33 mediated growth inhibition of YfkO M1 variants. S33dependent growth inhibition in 7NT was monitored by measuring strain turbidity $\left(O D_{600}\right)$ pre- and post-four hour incubation with a two-fold dilution series of S33, with a top concentration of $45 \mu M$ S33 to conserve drug stocks. Percentage growth relative to unchallenged controls was determined and used to calculate the concentration at which 50\% growth inhibition was seen using GraphPad Prism 7.0. Data are the average of 3 biological replicates $\pm S D$.

\subsubsection{YcnD_Bs and YfkO_Bs round 2 error-prone library creation and screening}

\subsubsection{Creation of libraries and effects of niclosamide selection}

During the second round of YcnD_Bs directed evolution, the low-expressing and high-expressing pUCX plasmid issues had not yet been resolved, and therefore all YcnD_Bs variants (cloned into high-expressing pUCX) were again compared to wild-type YcnD_Bs in low-expressing pUCX. The leading four unique variants from the YcnD and YfkO M1 libraries respectively were used as a mixed template for a second round of epPCR using the GeneMorphII polymerase. The amplified epPCR were cloned into highexpressing pUCX and transformed into the $E$. coli SOS-R4 strain, and sequence analysis of ten random variants from each library (designated the YcnD M2 and YfkO M2 libraries from here on) confirmed an average of three base-pair mutations (generally resulting in three amino acid substitutions) had been introduced into each variant.

A number of these mutations were discovered to be the result of DNA shuffling from M1 templates e.g. a YcnD variant was recovered that contained both the L128F and G223D amino acid substitutions, each of 
which had occurred in different M1 variants used as templates. This effect has been observed by other members in the Ackerley lab, and was postulated to be caused by low processivity of the GeneMorphII low-fidelity polymerase, leading to its dissociation from the DNA strand prior to full extension of the gene, and subsequent self-priming of overlapping sequences from different M1 templates. This would lead to a DNA shuffling outcome in a manner similar to Staggered Extension PCR, which uses very short extension times to force incomplete strand amplification and then cross-strand priming during successive cycles of PCR (Zhao and Zha, 2006). In total, the $y c n D \mathrm{M} 2$ library had 1.8 million variants, which was reduced to 1.2 million following selection on LB agar plates containing $0.5 \mu \mathrm{M}$ niclosamide (66\% selection). Due to difficulties in cloning of the $y f k O \mathrm{M} 2$ library and time constraints from pre-arranged flow cytometry bookings, the Round $2 y f k O$ library contained only 72,000 variants. This was reduced to 7,200 following selection using $0.5 \mu \mathrm{M}$ niclosamide (10\% selection).

\subsubsection{Fluorescence-activated cell sorting (FACS) of YcnD_Bs and YfkO_Bs M2 libraries}

In an attempt to further improve flow cytometry selection of the most active YcnD and YfkO M2 library variants, a double flow cytometry sort was conducted, as it had been previously shown by our research group to greatly enhance selection of active nitroreductase variants (Section 4.1.2; Copp et al.,(2014)). For the first sort, sub-cultures of YcnD_Bs and YfkO_Bs M2 libraries were challenged with $5 \mu \mathrm{M}$ S33.

As per Section 4.3.3.1, the pooled fluorescence of these libraries was measured in a plate reader, along with the wild-type enzyme controls (the wild-type control for the YcnD library unknowingly being YcnD_Bs in lowexpressing pUCX), a control comprising a mixed population of the four M1 variants used as template for the M2 library, and an empty pUCX control (Figure 4-10A). 
The pooled fluorescence of the YcnD M2 library was nearly two-fold lower than that of wild-type YcnD_Bs, which suggested a large proportion of the library had worse activity with S33 than YcnD_Bs. As a whole, the YfkO M2 library only exhibited a slightly lower fluorescence than wild-type YfkO_Bs.

A

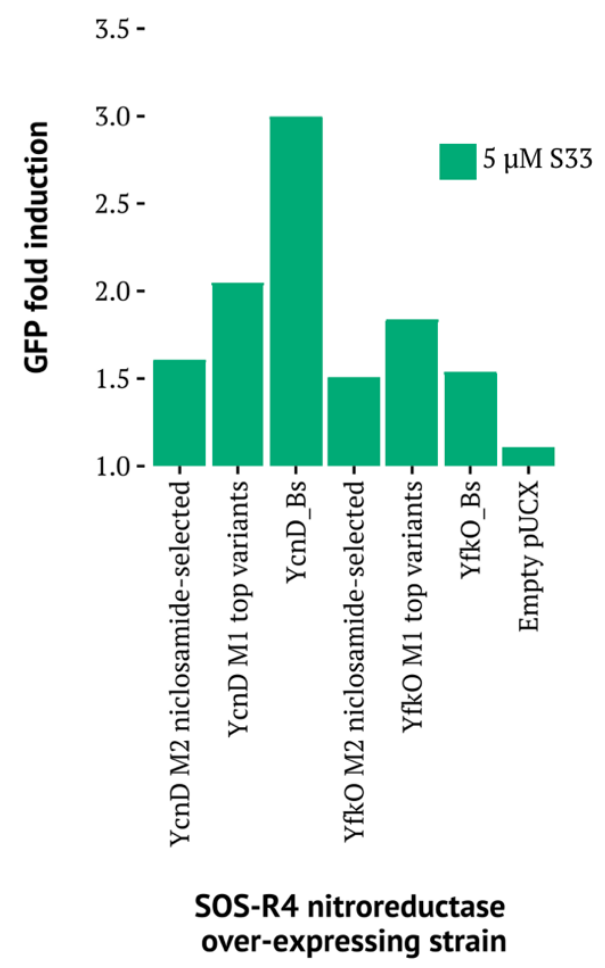

B

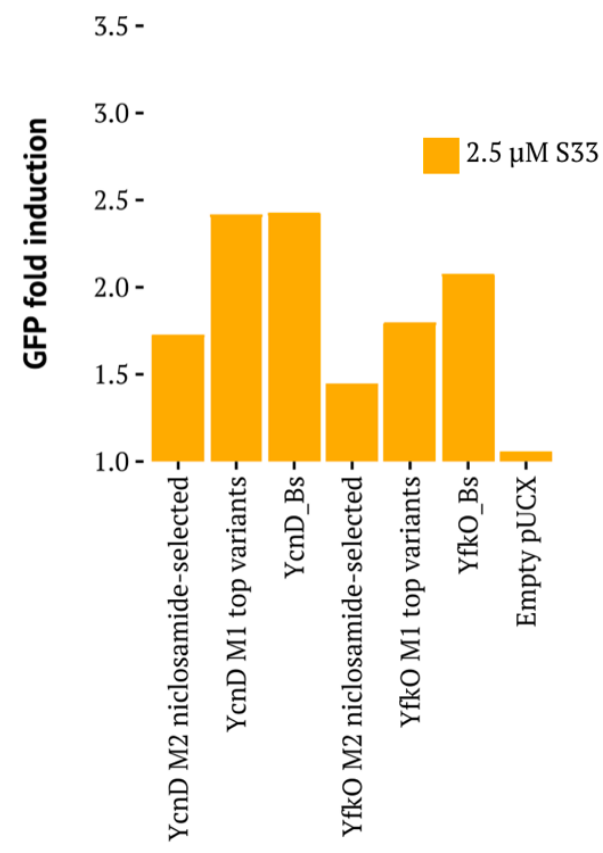

SOS-R4 nitroreductase over-expressing strain

Figure 4-10 S33 mediated induction of the GFP SOS response in E. coli SOS-R4 $Y \subset n D$ and $Y f k O$ library nitroreductase over-expression strains. A) GFP response of YcnD M2 and YfkO M2 libraries (and controls) following 4 hour challenge with 5 $\mu M S 33$ in 96-well microplates, immediately prior to first FACS sort. Fold induction is derived from the fluorescence recorded on drug exposure divided by that of unchallenged control wells. B) GFP response of FACS-sorted YcnD M2 and YfkO M2 libraries (and controls) following 4 hour challenge with $2.5 \mu M$ S33 in 96-well microplates, immediately prior to second FACS sort. Fold induction was derived from the fluorescence recorded on drug exposure divided by that of unchallenged control wells.

A $10 \mu \mathrm{L}$ sub-culture of each library and each wild-type control was diluted in M9 media and the most strongly GFP expressing cells were then selected by FACS, with 10,000 YcnD M2 and 10,000 YfkO M2 variants collected within 
gates that demarked the top 10\% of wild-type YcnD_Bs and YfkO_Bs events, respectively (flow cytometry plots not shown). The reason a high number of $y f k O$ variants was collected relative to the overall library size was to ensure multiple duplicates of the best variants and thereby ensure those variants would be well-represented in the library prior to the second sort.

Unlike FACS conducted on the Round 1 libraries (Section 4.3.2.2), these events were not sorted into 384-well microplates, but rather into a pooled collection in $10 \mathrm{~mL}$ of recovery media. This allowed for the collection of $>10$ fold more variants in the same period of time, albeit with the disadvantage that the percentage recovery could no longer be determined with a high degree of accuracy. Following collection, clones were left to recover overnight, after which the cultures were mixed 1:1 with $80 \%$ glycerol and stored at $-80^{\circ} \mathrm{C}$ until the second sort.

The following week the second enrichment sort was conducted, with subcultures of single-sorted YcnD and YfkO M2 libraries challenged with $2.5 \mu \mathrm{M}$ S33. At this lower concentration of S33, the pooled fluorescence of the single-sorted YcnD M2 library (Figure 4-10 B) exhibited a similar fluorescence to that displayed by the unsorted YcnD M2 library challenged with $5 \mu$ M S33 (Figure 4-10 A), suggesting that the first sort had indeed been successful in enriching for enzymes more active with S33. A similar phenomenon was observed with the YfkO M2 library; however in each case the fluorescence of the YcnD and YfkO M2 single-sorted libraries was still slightly lower than that of the wild-type parent enzyme control.

During the second sort, the 20,000 most fluorescent events for each library were collected within gates that demarked the top $10 \%$ of fluorescent events of each library. As described previously in this section, due to the low recovery rates seen with the sorted YfkO M1 library and the small library size of the YfkO M2 library, a large number of YfkO M2 events relative to the number of unique variants was collected to ensure that that the most active enzymes would be well represented within the YfkO M2 pool. 
These events were collected and plated on LB agar plates, and 7,500 YcnD M2 variants and 4,300 YfkO M2 variants were recovered (38\% and 22\% recovery rates respectively). Due to limited stocks of S33, only three 96-well plates per library were subjected to further screening using the GFP SOS assay (270 variants in total from each library). Each plate screened also contained the wild-type parent enzyme, pooled M1 variant enzymes, and empty pUCX controls in SOS-R4 cells.

\subsubsection{Screening of FACS-sorted YcnD and YfkO M2 libraries using the GFP SOS assay}

The initial screening for improved S33-activating variants from doublesorted YcnD and YfkO M2 libraries was conducted using the GFP SOS assay and $2.5 \mu \mathrm{M}$ S33, with each 96-well plate screened once (two technical replicates; results not shown). The lead 18 candidates from each plate were then picked into the inner wells of one 96-well plate, one for the YcnD M2 library and one for the YfkO M2 library, for more detailed second-tier screens, along with wild-type parent enzyme, pooled M1 variant enzymes, and empty pUCX controls on each plate.

The second-tier YfkO M2 plate was screened again using $2.5 \mu \mathrm{M}$ S33, whereas substantial growth inhibition observed from initial YcnD M2 plate screens (not shown) led us to drop the challenge concentration down to 1.25 $\mu$ M S33 for the second-tier YcnD M2 plate. Each plate was screened in triplicate (3 biological replicates; Figures 4-11 and 4-12).

The YcnD M2 variants most active with S33 exhibited a 1.3-fold increase in fluorescence over wild-type YcnD_Bs in low-expressing pUCX, whereas the most active YfkO M2 variants showed a 1.7-fold increase in fluorescence over wild-type YfkO_Bs. 


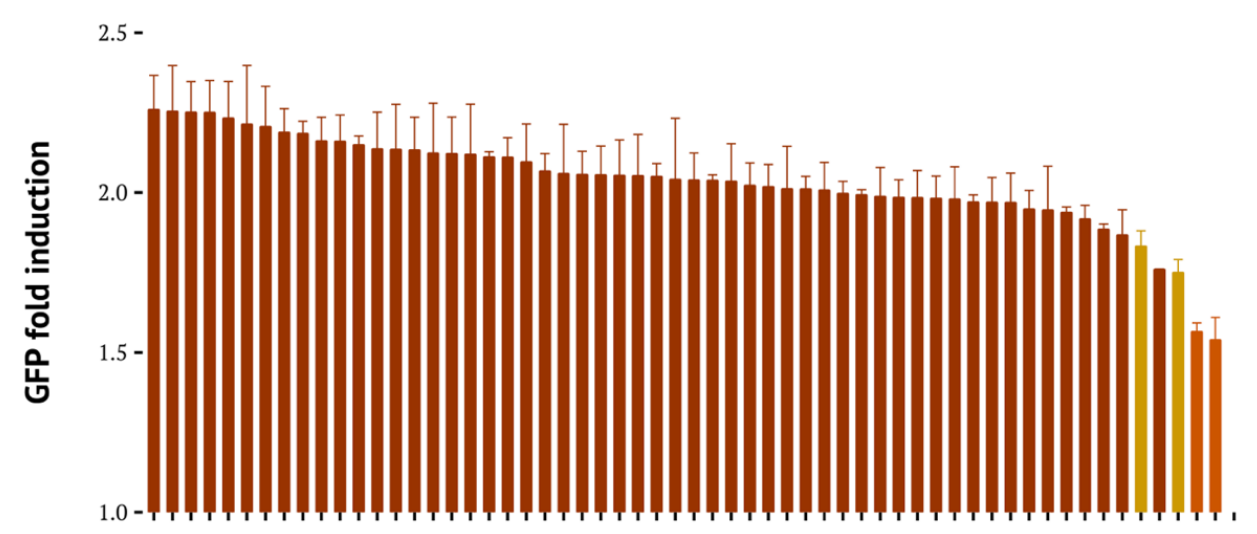

SOS-R4 nitroreductase overexpressing strain

Figure 4-11 Second-tier screening of S33 mediated induction of fluorescence in E. coli SOS-R4 cells over-expressing either native YcnD, lead YCnD M1 library variants, empty pUCX or YcnD M2 libraries post-FACS, challenged with $1.25 \mu \mathrm{M}$ S33. Over-expression strains were grown in 96-well microplates and challenged with $1.25 \mu M S 33$ for four hours prior to quantification of the SOS response by measurement of GFP fluorescence. Fold induction is derived from the fluorescence recorded on drug exposure divided by that of unchallenged control wells. Data are the average of 3 biological replicates \pm SEM. Controls include wild-type YcnD_Bs (yellow) and mixed YcnD_Bs M1 template variants (orange), with YcnD M2 variants shown in brown.

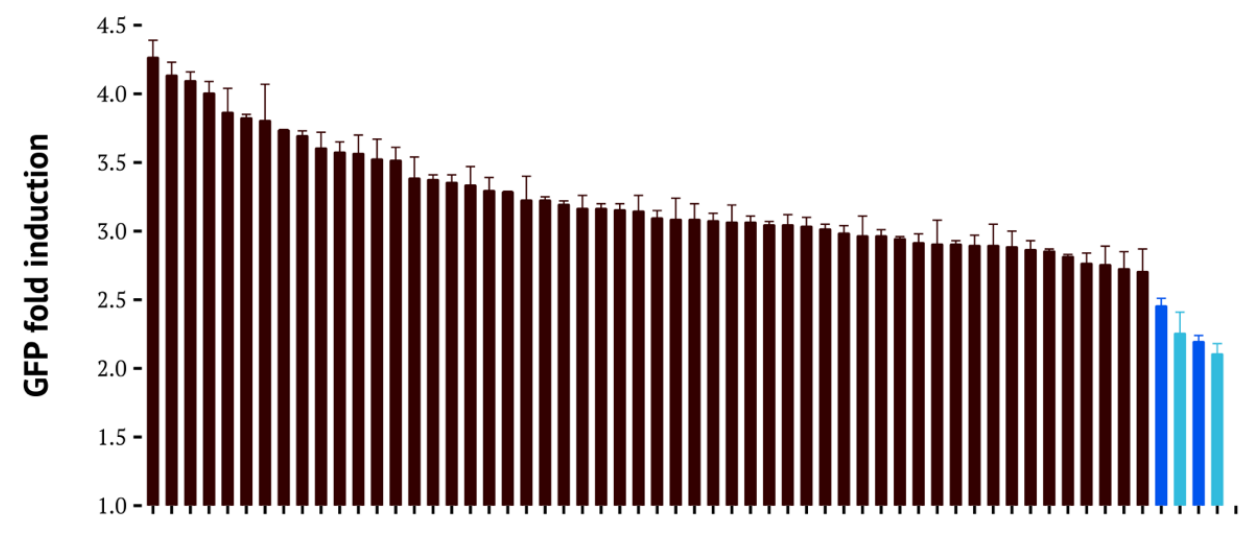

SOS-R4 nitroreductase overexpressing strain

Figure 4-12 Second-tier screening of S33 mediated induction of fluorescence in E. coli SOS-R4 cells over-expressing either native YfkO, lead YfkO M1 library variants, empty pUCX or YfkO M2 libraries post-FACS, challenged with $2.5 \mu \mathrm{M}$ S33. Overexpression strains were grown in 96-well microplates and challenged with 2.5 $\mu M S 33$ for four hours prior to quantification of SOS response by measurement of GFP fluorescence. Fold induction is derived from the fluorescence recorded on drug exposure divided by that of unchallenged control wells. Data are the average of 3 biological replicates \pm SEM. Controls include wild-type YfkO_Bs (aqua) and mixed YfkO_Bs M1 template variants (dark blue), with YfkO M2 variants shown in black. 


\subsubsection{Growth inhibition (IC $C_{50}$ ) assays with YcnD and YfkO M2 libraries and S33}

As discussed previously in Section 4.3.2.4, more sensitive growth inhibition $\left(\mathrm{IC}_{50}\right)$ assays with the leading $\mathrm{YcnD}$ and $\mathrm{YfkO} \mathrm{M} 2$ variants were initially performed in the SOS-R4 strain. However the subsequent discovery that the pANODuet-1:GFP plasmid contributed to the sensitising of SOS-R4 cells to lethal concentrations of S33 (see Section 4.3.4) caused us to re-transform each variant into the $E$. coli $7 \mathrm{NT}$ strain (lacking the GFP plasmid) for $\mathrm{IC}_{50}$ assays, to determine the true effect of each variant on sensitising $E$. coli to lethal concentrations of S33. $\mathrm{IC}_{50}$ values for these variants were determined by screening across a dilution series of up to $70 \mu \mathrm{M}$ S33 (Tables 4-3 and 4-4). The activities of variants were compared to wild-type controls; by this stage of the research the presence of low-expressing YcnD_Bs had been discovered, and both $\mathrm{YcnD}$ and $\mathrm{YfkO}$ variants were compared appropriately to wild-type enzymes in high-expressing pUCX.

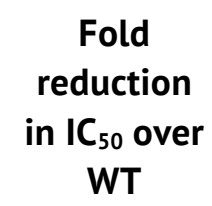

\begin{tabular}{c|c|c|c} 
Enzyme & IC $_{50}(\boldsymbol{\mu M})$ & YcnD_Bs & aa substitutions \\
\hline YcnD_M1 1_19 & $15.3 \pm 2.8$ & 1.2 & N141D/Q178R/Q222H \\
YcnD_M1 1_22 & $15.5 \pm 1.8$ & 1.2 & G223D \\
YcnD_M2 3_16 & $15.6 \pm 0.5$ & 1.2 & D71V/G223D \\
YcnD_M2 3_80 & $16.8 \pm 2.0$ & 1.1 & L128F/G223D \\
YcnD_M1 1_18 & $16.9 \pm 0.8$ & 1.1 & Q32R/E147G \\
YcnD_M1 1_31 & $16.9 \pm 0.6$ & 1.1 & L128F \\
High-expressing & $18.5 \pm 1.6$ & 1.0 & - \\
YcnD_Bs & & & Q32R/N141D/Y183H \\
YcnD_M2 3_79 & $18.6 \pm 0.5$ & 1.0 & /Q222H \\
YcnD_M2 2_14 & $18.9 \pm 1.3$ & 1.0 & Q35L/E62K/N141D \\
Empty pUCX & $>>70$ & - &
\end{tabular}

Table 4-3 I $C_{50}$ values for S33 mediated growth inhibition of $Y c n D M 1$ and M2 variants. S33-dependent growth inhibition in 7NT was monitored by measuring strain turbidity $\left(\mathrm{OD}_{600}\right)$ pre- and post-four hour incubation across a two-fold dilution series of S33, with a top concentration of $70 \mu M$ S33 to conserve drug stocks. Percentage growth relative to unchallenged controls was determined and used to calculate the concentration at which $50 \%$ growth inhibition was seen using GraphPad Prism 7.0. Data are the average of 3 biological replicates $\pm S D$. 


\begin{tabular}{|c|c|c|c|}
\hline Enzyme & $I C_{50}(\mu \mathrm{M})$ & $\begin{array}{c}\text { Fold } \\
\text { reduction } \\
\text { in } \mathrm{IC}_{50} \\
\text { over WT } \\
\text { YfkO_Bs }\end{array}$ & aa substitutions \\
\hline YfkO_M2 3_62 & $18.1 \pm 2.3$ & 2.6 & F82L/M110I \\
\hline YfkO_M2 1_32 & $19.2 \pm 2.3$ & 2.5 & F82L/T122R \\
\hline YfkO_M2 1_88 & $19.6 \pm 3.5$ & 2.4 & F14L/F82L/K94T/T122M/L133H \\
\hline YfkO_M1 3_44 & $22.5 \pm 4.4$ & 2.1 & $\mathrm{~T} 122 \mathrm{R}$ \\
\hline YfkO_M1 1_10 & $27.5 \pm 3.4$ & 1.7 & F82L \\
\hline YfkO_M1 3_2 & $28.2 \pm 3.9$ & 1.7 & M110I \\
\hline YfkO_M1 3_20 & $29.2 \pm 4.8$ & 1.6 & L133H \\
\hline YfkO_Bs & $47.1 \pm 6.9$ & 1.0 & \\
\hline Empty pUCX & $>>70$ & - & \\
\hline
\end{tabular}

Table 4-4 I $C_{50}$ values for $S 33$ mediated growth inhibition of YfkO M1 and M2 variants. S33-dependent growth inhibition in 7NT was monitored by measuring strain turbidity $\left(O D_{600}\right)$ pre- and post-four hour incubation across a two-fold dilution series of S33, with a top concentration of $70 \mu M S 33$ to conserve drug stocks. Percentage growth relative to unchallenged controls was determined and used to calculate the concentration at which 50\% growth inhibition was seen using GraphPad Prism 7.0. Data are the average of 3 biological replicates $\pm S D$.

All YcnD_Bs M1 and M2 variants exhibited minimal improvements in their ability to sensitise E. coli to S33 over high-expressing YcnD_Bs, due to their previous comparison to YcnD_Bs in low-expressing pUCX during FACS selection and subsequent screening.

However, all lead variants from the YfkO M2 library showed significant improvements in activity over wild-type $\mathrm{YfkO}_{-} \mathrm{Bs}$, with an over 2.4-fold improvement in sensitising 7NT cells to S33 over YfkO_Bs. This demonstrated that successive rounds of random mutagenesis and FACS selection of improved variants had been moderately successful in improving YfkO_Bs activity with S33. The YfkO M2 variants 3_62 and 1_32 each contained combinations of single mutations found in YfkO M1 variants (F82L and M110I, and F82 and T122R respectively), suggesting an additive or synergistic effect of these combined substitutions on activity with S33. The third YfkO M2 variant, 1_88, contained five amino acid substitutions (F14L/F82L/K94T/T122M/L133H), of which three had been generated during the second round of error-prone PCR. One of these substitutions involved a 
hydrophobic Met in place of Thr at residue 122, whereas in the YfkO M1 variants 3_44 and M2 1_32 a substitution to the positively charged Arg was observed, perhaps suggesting that simply a larger amino acid at this position improved the activity of YfkO_Bs with S33.

\subsubsection{Activity of YfkO M2 variants with next-generation GDEPT prodrugs and PET imaging probes}

\subsection{Background}

At this stage of my research, our collaborators at the ACSRC and the Universities of Maastricht and Nottingham had shown interest in NfsB variants that exhibited dual activity with the next-generation GDEPT prodrug SN36506 (chemical structure shown in Figure 4-13) as well as the next-generation PET-capable imaging probe SN37819, a single-fluorine (and hence easier to radio-label with ${ }^{18} \mathrm{~F}$ ) analogue of S33 (Anderson et al., 2014). This interest was based on our ongoing multi-centre collaboration seeking to take nitroreductase-armed Clostridium sporogenes to human GDEPT clinical trials. In Clostridium-directed enzyme prodrug therapy (CDEPT), spores of nitroreductase-armed Clostridia are injected into a patient, whereupon the obligate anaerobe germinates and colonises hypoxic and necrotic areas of tissue in tumour cells (Mowday et al., 2016a). The hypoxic tissues then become sensitive to an administered prodrug such SN36506. To 'arm' C. sporogenes, our collaborators have developed novel methods for the chromosomal integration of nitroreductase genes (Heap et al., 2007; Kuehne and Minton, 2012).

Despite these advances, $C$. sporogenes had proven unable to functionally express any of our four lead NfsA-family nitroreductases (YcnD_Bs, NfrA_Bs, NfsA_Ec and YcnD_Bli), whereas five NfsB-family nitroreductases (NfsB_Nme, NfsB_Ec, YfkO_Bs, YfkO_Bli, NfsB_Vv) were tolerated and functional. This suggested that some innate activity associated with the NfsA family might be detrimental to $C$. sporogenes, and encouraged our primary focus on NfsB enzymes. Unfortunately, as illustrated in Figure 
3-11, only a subset of NfsB-family nitroreductases are active with 5nitroimidazole PET probes and none of the five NfsB enzymes tested at the time were found to be effective with both 5-nitroimidazole probes and the lead prodrug SN36506.

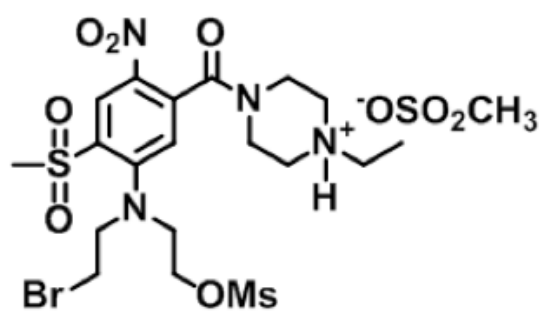

Figure 4-13 Chemical structures of the prodrug SN36506.

SN36506 is a nitrogen mustard prodrug that lacks the dose-limiting offmechanism activation by endogenous human aldo-keto reductase $1 \mathrm{C} 3$ exhibited by the previous lead analogue, PR-104A (Guise et al., 2010). SN36506 has the further advantage of being orally available (McKeage et al., 2011). Unfortunately, SN36506 is generally more resistant than PR-104A to activation by bacterial nitroreductases (Dr David Ackerley, personal communication).

The leading S33-improved YfkO M1 and M2 variants described in Sections 4.3.2 and 4.3.3 were tested for their ability to activate both SN36506 and the S33 analogue SN37819. These enzymes were first benchmarked by GFP-SOS screening for SN36506 activity alongside a panel of NfsA and NfsB family enzymes from the 58 nitroreductase library (Figure 4-14). This revealed that YfkO M2 variants M2 1_88 and M2 3_62 were able to induce higher SOS responses in E. coli SOS-R4 cells than wild-type YfkO_Bs following challenge with $150 \mu \mathrm{M}$ SN36506, suggesting that they exhibited improved activity with this prodrug. 


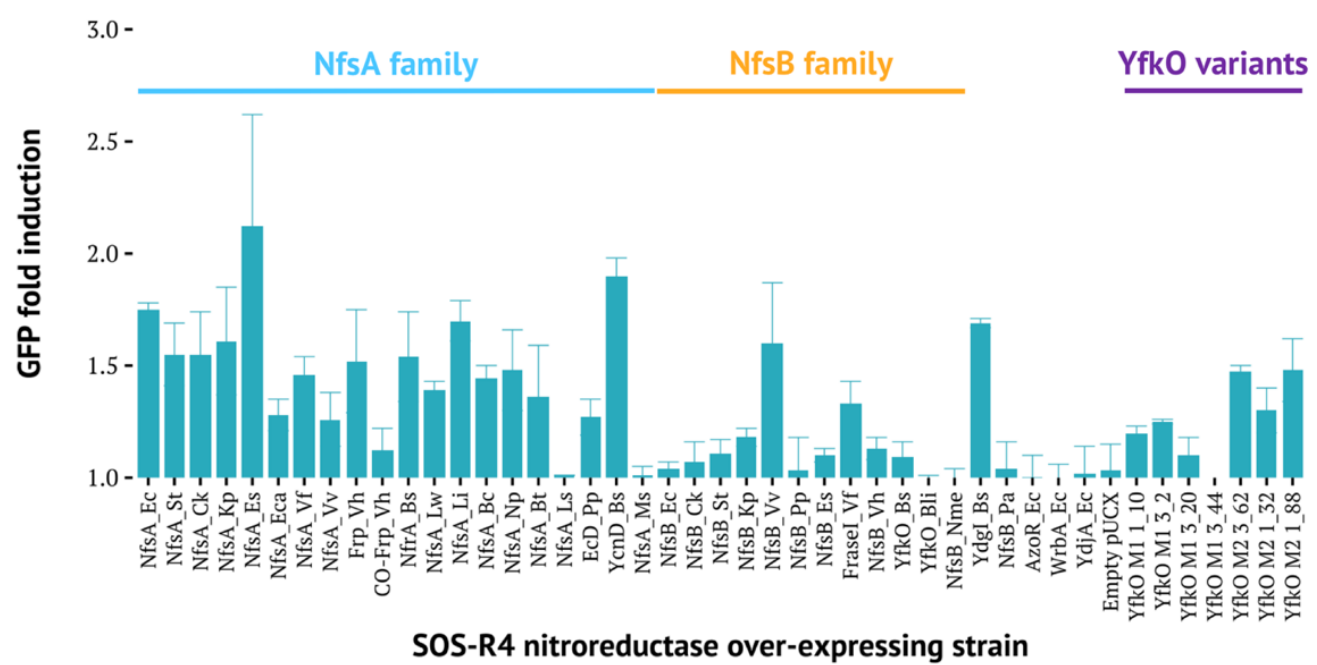

Figure 4-14 S33 mediated induction of fluorescence in E. coli SOS-R4 nitroreductase over-expression strains, challenged with $150 \mu M$ SN36506. Overexpression strains were grown in 384-well microplates and challenged with 150 $\mu M S N 36506$ for six hours prior to quantification of SOS response by measurement of GFP fluorescence. Fold induction is derived from the fluorescence recorded on drug exposure divided by that of unchallenged control wells. Data are the average of 2 biological replicates \pm SEM. Data was generated by Dr Elsie Williams in the Ackerley lab and copied with permission.

\subsection{Growth inhibition (IC 50 assays) of YfkO M2 variants with prodrugs and PET imaging probes}

Based on the preliminary results described in Section 4.3.3.1.1, we conducted a comprehensive evaluation of the activity of YfkO M2 variants 1_88 and 3_62 with a panel of substrates of interest to the $C$. sporogenes collaborative team. These substrates included the prodrugs SN36506 and PR-104A (the previous lead prodrug candidate) as well as the two 5nitroimidazole imaging probes, S33 and SN37819. In parallel with the analysis of the two evolved YfkO M2 variants, other nitroreductases that were evaluated included several other nitroreductases that 1) had already been shown to express both stably and functionally in C. sporogenes (NfsB_Ec, NfsB_Nme from Neisseria meningitidis, and YfkO_Bli from $B$. licheniformis); 2) had been tested but not yet found to express both stably and functionally in C. sporogenes (NfsA_Ec, YcnD_Bs, NfrA_Bs); or 3) were additional WT or evolved enzymes generated by other Ackerley lab members 
that were now being considered as additional candidates for expression and activity trials in C. sporogenes (YfkO_Bs, NfsA_Es, YdgI_Bs, NfsB_Vv, and a site-targeted double-mutant of NfsB_Vv, F70A/F108Y, which had been designed to have enhanced activity with 5-nitroimidazole probes).

To evaluate the activity of these nitroreductases with the different substrates I determined their ability to sensitise $E$. coli $7 \mathrm{NT}$ strains to lethal concentrations of these compounds in growth inhibition ( $\left.\mathrm{IC}_{50}\right)$ assays, across appropriate dilution series of each compound (Table 4-5). Due to a previously observed phenomenon that activated SN36506 is effluxed from $7 \mathrm{NT}$ cells (even with the knock-out of the tolC efflux pump gene), which limits the ability to measure nitroreductase-mediated sensitisation of $E$. coli to lethal concentrations of SN36506 (Dr Elsie Williams, personal communication), $10 \mu \mathrm{M}$ of the efflux pump inhibitor phenylalanine-arginine beta-naphthylamide (PA $\beta N$; Lomovskaya et al., 2001) was also included in SN36506 challenge media.

Out of the complete panel of 13 nitroreductases, YfkO M2 1_88 and 3_62 showed moderate levels of activity with SN36506, and exhibited similarly low levels of activity with PR-104A as wild-type YfkO_Bs. Similar to their previous characterisation in Table 4-4, both sensitised cells to $>2.2$-fold lower concentrations of S33 than YfkO_Bs, and also >5-fold lower concentrations of the 5-nitroimidazole probe SN37819. However ultimately the NfsB_Vv variant F70A/F108Y was selected as the most promising NfsB orthologue for further analysis by our collaborators due to its ability to sensitise 7NT to >2-fold lower concentrations of SN36506 than both YfkO M2 variants. As well as slightly improving its activity with this prodrug, the amino acid substitutions introduced into $\mathrm{NfsB}_{-} \mathrm{Vv}$ enabled it to be capable of activating both S33 and SN37819, albeit with over 2-fold higher $\mathrm{IC}_{50}$ values than either YfkO M2 variant. This variant is currently being integrated into the $C$. sporogenes chromosome under control of a panel of different promoters to optimise its expression, after which its potential for SN36506based CDEPT will be evaluated in mouse xenograft models. 


\begin{tabular}{|c|c|c|c|c|}
\hline $\begin{array}{l}\text { Nitroreductase } \\
\text { variant }\end{array}$ & $\begin{array}{l}\text { SN36506 } \\
I_{50}(\mu M)\end{array}$ & $\begin{array}{l}\text { PR-104A } \\
I C_{50}(\mu M)\end{array}$ & $\begin{array}{l}\text { SN37819 } \\
I_{50}(\mu M)\end{array}$ & $\begin{array}{c}\text { S33 IC } 50 \\
(\mu \mathrm{M})\end{array}$ \\
\hline YcnD_Bs & $320 \pm 48$ & $951 \pm 89$ & $8 \pm 2$ & $17 \pm 2$ \\
\hline NfsB_Vv F708/108Y & $369 \pm 81$ & $1240 \pm 90$ & $46 \pm 12$ & $75 \pm 15$ \\
\hline NfrA_Bs & $404 \pm 56$ & $969 \pm 112$ & $30 \pm 5$ & $34 \pm 9$ \\
\hline NfsB_Vv & $424 \pm 54$ & $1440 \pm 160$ & $>250$ & $>150$ \\
\hline YdgI_Bs & $425 \pm 80$ & $1130 \pm 140$ & $35 \pm 7$ & $51 \pm 7$ \\
\hline NfsA_Ec & $615 \pm 28$ & $1110 \pm 80$ & $34 \pm 7$ & $38 \pm 2$ \\
\hline YfkO M2 1_88 & $698 \pm 5$ & $1560 \pm 150$ & $17 \pm 5$ & $19 \pm 1$ \\
\hline YfkO M2 3_62 & $807 \pm 85$ & $1780 \pm 110$ & $20 \pm 4$ & $19 \pm 1$ \\
\hline NfsB_Ec & $>1000$ & $1420 \pm 120$ & $>250$ & $>150$ \\
\hline NfsB_Nme & $>1000$ & $1350 \pm 70$ & $>250$ & $>150$ \\
\hline YfkO_Bli & $>1000$ & $1130 \pm 60$ & $>250$ & $53 \pm 8$ \\
\hline YfkO_Bs & $>1000$ & $1640 \pm 20$ & $111 \pm 29$ & $43 \pm 1$ \\
\hline NfsA_Es & $>1000$ & $1430 \pm 40$ & $133 \pm 52$ & 112 \\
\hline Empty pUCX & $>1000$ & $>2000$ & $>250$ & $>150$ \\
\hline
\end{tabular}

Table 4-5 I 50 values of prodrug or imaging probe mediated growth inhibition of candidate nitroreductases. Compound-dependent growth inhibition in 7NT was monitored by measuring strain turbidity $\left(\mathrm{OD}_{600}\right)$ pre- and post-four hour incubation with a two-fold dilution series of SN36506 (top concentration of 1 $m M, 10 \mu M P A \beta N$ included in challenge media), PR-104A (top concentration of $2 \mathrm{mM}$ ), SN37819 (top concentration of $250 \mu M$ ), or $S 33$ (top concentration of $250 \mu \mathrm{M})$. Percentage growth relative to unchallenged controls was determined and used to calculate the concentration at which 50\% growth inhibition was seen using GraphPad Prism 7.0. Data are the average of at least 2 biological replicates $\pm S D$. IC $C_{50}$ values are colour coded green to yellow to red based on the activity of the nitroreductase with the compound (green= highly active, yellow = moderate activity, red = low activity). Enzymes are ranked in order of their activity with SN36506.

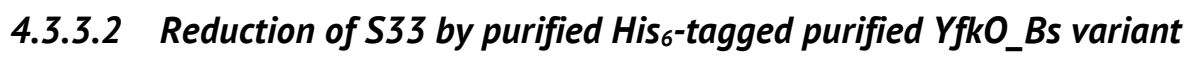 proteins}

Although the activities of the YfkO M2 variants 1_88 and 3_62 with SN36506 were insufficiently compelling to warrant their selection as candidates for SN36506-based CDEPT, they nevertheless performed well with the proprietary 5-nitroimidazole PET probe SN37819. This observation suggests that the YfkO M2 variants 1_88 and 3_62 might still offer genuine promise for PET imaging of $C$. sporogenes or other GDEPT vectors, using either 5nitroimidazole probe. Unfortunately our stock of SN37819 was too limiting 
to allow a comprehensive kinetic evaluation at a purified protein level to be performed. Instead, confirmation that these variants at least exhibited improved activity with S33 in vitro was conducted following their cloning into the His $_{6}$-tag expression vector pET28a+, and in vitro kinetics were measured for the purified proteins with S33, utilising the glucose oxidasecatalase oxygen scavenging system described previously in Section 3.3.2.4.

The YfkO M2 variant 1_88 showed a 4-fold improvement in $k_{\text {cat }} / K_{M}$ over wildtype YfkO_Bs, with an 8-fold reduction in $K_{M}$ but also a 2-fold decrease in $k_{\text {cat. }}$ Likewise, YfkO M2 3_62 showed over 2-fold increase in $k_{\text {cat }}$ and a 3-fold decrease in $K_{M}$ over YfkO_Bs, with a 6.6-fold improvement in $k_{\text {cat }} / K_{M}$ overall. This demonstrated that directed evolution of YfkO_Bs for improved S33 activation had successfully generated variants exhibiting improved catalytic activity with this PET imaging probe; and in the case of the latter enzyme, this was in part due to an improvement in $k_{\text {cat }}$.

\begin{tabular}{|c|c|c|c|c|}
\hline Enzyme & $\boldsymbol{k}_{\text {cat }}\left(\mathbf{s}^{-1}\right)$ & $K_{M}(\mu \mathrm{M})$ & $\begin{array}{c}k_{c a t} / K_{M} \\
\left(M^{-1} \cdot s^{-1}\right)\end{array}$ & $\begin{array}{c}\text { NADPH } \\
\text { oxidase } \\
\text { activity }(\mu \mathrm{M} \\
\text { NADPH. } \mu \mathrm{M} \\
\text { enzyme }^{-1} \cdot \mathrm{s}^{-1} \text { ) }\end{array}$ \\
\hline $\begin{array}{c}\text { YfkO M2 } \\
1 \_88\end{array}$ & $1.4 \pm 0.2$ & $600 \pm 160$ & $2,300 \pm 700$ & $0.05 \pm 0.00$ \\
\hline $\begin{array}{c}\text { YfkO M2 } \\
3 \_62\end{array}$ & $5.6 \pm 1.2$ & $1500 \pm 500$ & $3,700 \pm 1500$ & $0.10 \pm 0.00$ \\
\hline YfkO_Bs & $2.7 \pm 1.0$ & $4800 \pm 2400$ & $560 \pm 350$ & $0.12 \pm 0.00$ \\
\hline
\end{tabular}

Table 4-6 Michaelis-Menten kinetic parameters for YfkO M2 variants and S33. Initial rates of reduction at varying $S 33$ concentrations and a fixed concentration of excess NADPH $(250 \mu \mathrm{M})$ were monitored at $350 \mathrm{~nm}$, with rates at each $\mathrm{S33}$ concentration measured in at least triplicate. Apparent $K_{M}$ and $k_{\text {cat }}$ at $250 \mu M$ $N A D P H$ with a glucose oxidase-catalase oxygen scavenging system (12 units. $\mathrm{mL}^{-}$ ${ }^{1}$ glucose oxidase, 12 units. $\mathrm{mL}^{-1}$ catalase, $50 \mathrm{mM}$ glucose) were calculated using GraphPad 7.0. An extinction coefficient of $9,650 \mathrm{M}^{-1} \mathrm{~cm}^{-1}$ was used to determine rates of nitroreductase-mediated reduction of both S33 and NADPH and errors represent \pm SEM. NADPH oxidase activity shows enzyme-mediated NADPH oxidation in the absence of a glucose oxidase-catalase oxygen scavenging system, with an extinction coefficient of $4020 \mathrm{M}^{-1} \mathrm{~cm}^{-1}$ used. 


\subsubsection{Effects of pANO-Duet-1:GFP plasmid on sensitising E. coli to nitroreductase-induced growth inhibition}

As previously described in Sections 4.3.2.4 and 4.3.3.4, over the course of the research conducted in this chapter it was discovered that different biological replicates of SOS-R4 (GFP plasmid-containing) cells overexpressing the same nitroreductase exhibited substantially different $\mathrm{IC}_{50}$ assays following challenge with S33. This issue had not been previously observed by our research group with the SOS-R2 E. coli strain (containing a chromosomally integrated SOS-induced lacZ gene), and it was determined that either the plasmid-based reporter system or the GFP product was contributing to the differences in sensitivity. In the latter regard, previous studies have noted the toxic effects of wild-type GFP aggregate bodies in $E$. coli due to poor protein solubility (Cormack et al., 1996; Crameri et al., 1996), although the gfpmut3b gene that is present in pANODuet-1:GFP is generally regarded to be more soluble (Cormack et al., 1996). This section describes efforts to define the extent of the problem and the possible implications for directed evolution using the SOS-R4 reporter strain.

\subsubsection{Screening of GFP plasmid-containing or lacking E. coli strains with SOS- inducing or non-inducing nitroaromatic compounds}

We thus sought to test whether SOS-induced GFP production from the pANODuet-1:GFP plasmid was contributing to variability in growth inhibition of $E$. coli when exposed to lethal concentrations of nitroaromatic compounds. To achieve this, we looked at the variance in $\mathrm{IC}_{50}$ values from different biological replicates of cells over-expressing NfsA_Ec when exposed either to an SOS-inducing nitroaromatic prodrug (metronidazole) or a compound which did not induce the SOS response (the 2-nitroimidazole imaging probe HX4). More specifically, direct comparison between the same E. coli strain either lacking (7NT) or co-expressing the pANODuet-1:GFP plasmid (SOS-R4) and over-expressing NfsA_Ec was conducted. It was predicted the production of GFP following challenge with metronidazole in the GFP-expressing strain would contribute to more variable $\mathrm{IC}_{50}$ values than 
the same strain lacking the GFP plasmid. Alternatively, challenge with HX4 would result in minimal SOS-induced GFP production and non-variable IC $_{50}$ values between biological replicates of the GFP-expressing strain.

\subsubsection{Growth inhibition $\left(I C_{50}\right)$ assays with pUCX:NfsA_Ec in 7NT or SOS-R4 cells and metronidazole}

NfsA_Ec has been shown by the Ackerley lab to exhibit activity with the 5nitroimidazole prodrug metronidazole, and activated metronidazole strongly induces the SOS response in both E. coli SOS-R2 and SOS-R4 strains (Little, 2015; Prosser et al., 2010b). Growth inhibition assays were conducted with 19 different biological replicates of 7NT cells (no GFP plasmid) overexpressing NfsA_Ec, and 19 different biological replicates of SOS-R4 cells (co-expressing GFP plasmid) over-expressing NfsA_Ec, with a dilution series of up to $200 \mu \mathrm{M}$ metronidazole (Figure 4-15).

As anticipated, the standard deviation in $\mathrm{IC}_{50}$ values between biological replicates of NfsA_Ec over-expressing 7NT cells did not exceed 8\% of the average (102 $\mu \mathrm{M})$, suggesting that all biological replicates in this strain behaved similarly.

In contrast, a $40 \%$ standard deviation in $\mathrm{IC}_{50}$ values was observed between biological replicates of NfsA_Ec over-expressing SOS-R4 cells, with a much lower average IC $_{50}$ value of $69 \mu \mathrm{M}$. This indicated a substantial difference in cell sensitisation to metronidazole between biological replicates of SOS-R4 cells, and the difference in in $\mathrm{IC}_{50}$ values between $7 \mathrm{NT}$ and SOS-R4 cells was highly statistically significant ( $p$ value $<0.0001$ ). 


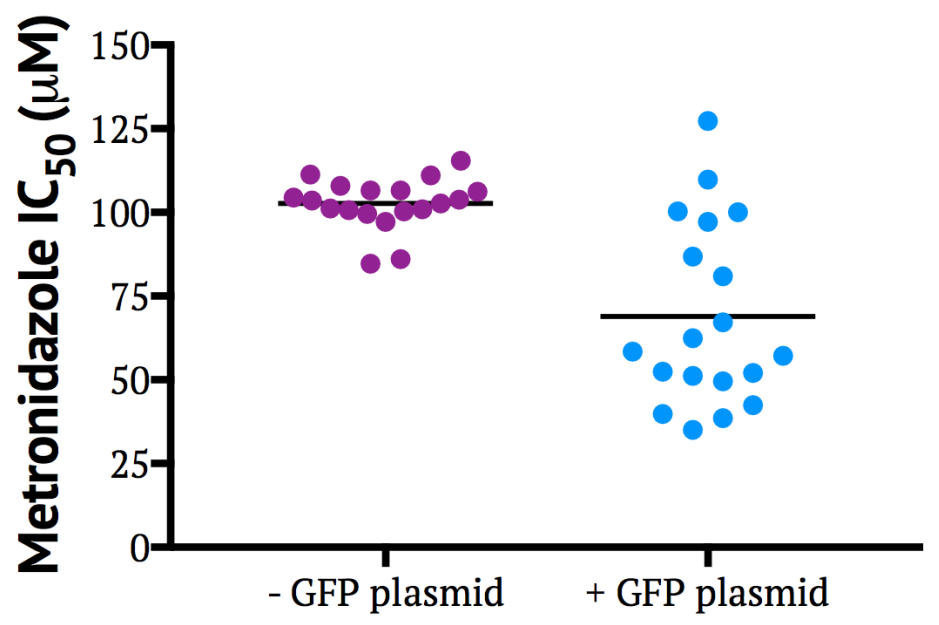

Figure 4-15 IC 50 values of metronidazole mediated growth inhibition of NfSA_Ec in 7NT cells with or without the pANODuet-1:GFP plasmid. 19 different biological replicates of E. coli 7NT (-GFP plasmid) bearing pUCX:NfSA_Ec (purple) and 19 different biological replicates of pUCX:NfSA_Ec in $7 N T$ co-expressing pANODuet-1:GFP (+GFP plasmid; blue) were subjected to metronidazoledependent growth inhibition, which was monitored by measuring strain turbidity $\left(O D_{600}\right)$ pre- and post-four hour incubation across a two-fold dilution series of metronidazole. Percentage growth relative to unchallenged controls was determined and used to calculate the concentration at which 50\% growth inhibition occurred, using GraphPad Prism 7.0. Data are the average of 3 replicates (error bars omitted for clarity). Differences between the mean $I C_{50}$ values in biological replicates of $7 N T$ or SOS-R4 were statistically significant when compared using unpaired Student's t-tests $-* * * * *=p$ value $<0.0001$.

Furthermore, during the measurement of cell turbidity at 4 hours in $\mathrm{IC}_{50}$ assays in Figure 4-15, the fluorescence of SOS-R4 cultures was also measured, and varying levels of background fluorescence in unchallenged wells (i.e. not metronidazole-induced GFP) between different cultures were revealed. To confirm that presence of different levels of background GFP was contributing to the wildly different $\mathrm{IC}_{50}$ values in clonal isolates of NfsA_Ecexpressing SOS-R4 strains, these background levels of GFP for each culture were linearly correlated with the metronidazole $\mathrm{IC}_{50}$ value for the same culture (Figure 4-16; IC 50 values taken from Figure 4-15). It should be noted that all NfsA_Ec-expressing SOS-R4 cultures exhibited similar foldincreases in fluorescence upon incubation with the lowest concentration of metronidazole used in these assays ( $25 \mu \mathrm{M}$ metronidazole, at which a 4.9- 
fold increase in fluorescence was observed with a standard deviation of $<10 \%$ between different cultures, results not shown).

There was a very strong correlation between background fluorescence levels and $\mathrm{IC}_{50}$ values of SOS-R4 pUCX:NfsA_Ec cultures $\left(\mathrm{R}^{2}=0.938\right.$, see Figure 4-16), indicating that cultures with higher background fluorescence (and hence more fluorescence upon challenge with lethal concentrations of metronidazole) exhibited lower $\mathrm{IC}_{50}$ values with metronidazole. This strongly suggested that the variable background levels of GFP across different cultures of the same nitroreductase variant played a substantial role in sensitising these cultures to lethal concentrations of an SOSinducing nitroaromatic compound.

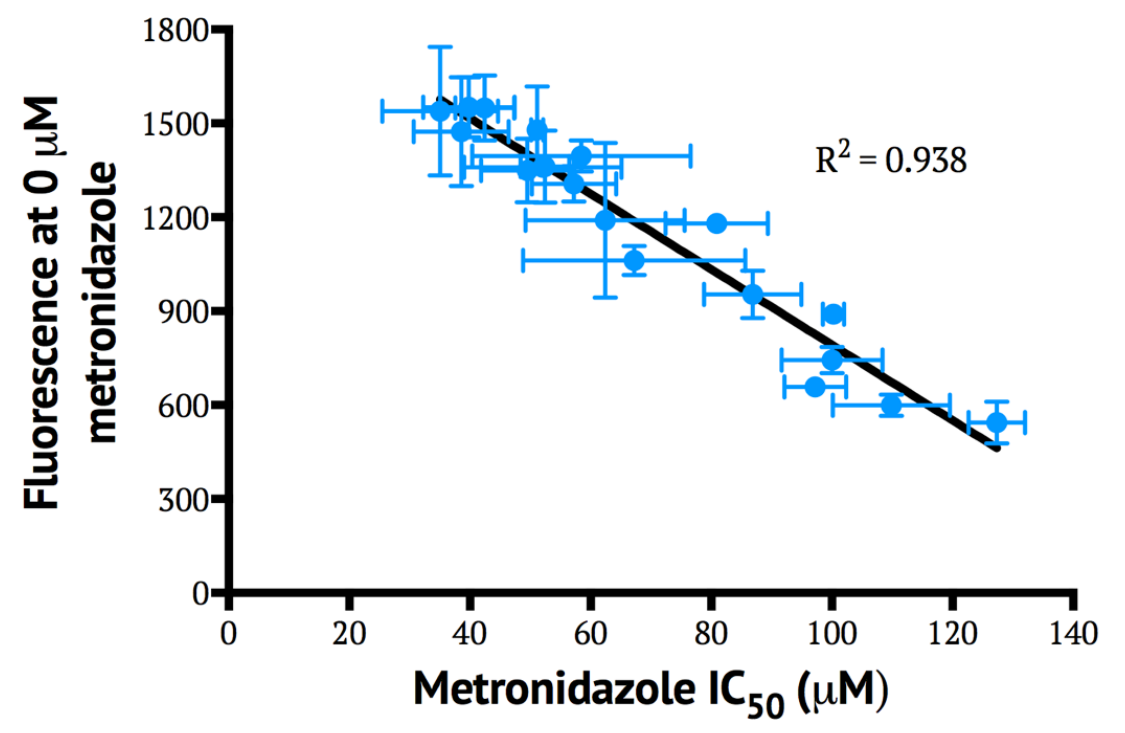

Figure 4-16 Linear correlation between background fluorescence levels and I $C_{50}$ growth inhibitory effects of metronidazole on SOS-R4 cells over-expressing pUCX:NfsA_Ec. IC $C_{50}$ values of 19 different biological replicates of SOS-R4 pUCX:Nfs__Ec cultures following challenge with up to $200 \mu M$ metronidazole in growth inhibition assays (data from Figure 4-15) were correlated with the observed fluorescence in unchallenged wells (containing $0 \mu M$ metronidazole) for the same culture in these assays. 


\subsubsection{Growth inhibition (IC $C_{50}$ ) assays with pUCX:NfsA_Ec in 7NT or SOS-R4 cells and $H X 4$}

Unlike metronidazole, the 2-nitroimidazole PET imaging probe HX4 has been shown by our lab to scarcely induce the $E$. coli SOS response in the SOS-R2 strain, irrespective of whether or not it is over-expressing an activating nitroreductase (Williams, 2013). As shown in Section 4.3.4.2, metronidazole-induced GFP production via the SOS response played a role in the varying $\mathrm{IC}_{50}$ values observed in different cultures of SOS-R4 cells overexpressing NfsA_Ec. As HX4 does not strongly induce the SOS response, we postulated that this variability in $\mathrm{IC}_{50}$ values would not be observed in different cultures of SOS-R4 cells over-expressing NfsA_Ec when challenged with lethal concentrations of the probe. $\mathrm{IC}_{50}$ assays (Figure 4-17) were conducted using a dilution series of up to $300 \mu \mathrm{M}$ HX4 for 19 different biological replicates of 7NT cells over-expressing NfsA_Ec and 19 different biological replicates of SOS-R4 over-expressing NfsA_Ec.

As predicted, no significant difference was observed between $\mathrm{IC}_{50}$ values of different biological replicates of 7NT or SOS-R4 cells over-expressing NfsA_Ec following challenge with HX4. Furthermore, the standard deviation in $\mathrm{IC}_{50}$ values between biological replicates of NfsA_Ec over-expressing SOSR4 cells did not exceed 14\% of the average, indicating that all 19 cultures behaved similarly when challenged with a non-SOS-inducing compound. This indicated that the effects of GFP on the variability of IC $_{50}$ values of nitroreductase variants in the SOS-R4 strain was only an issue when the cells were challenged with an SOS-inducing nitroaromatic substrate. 


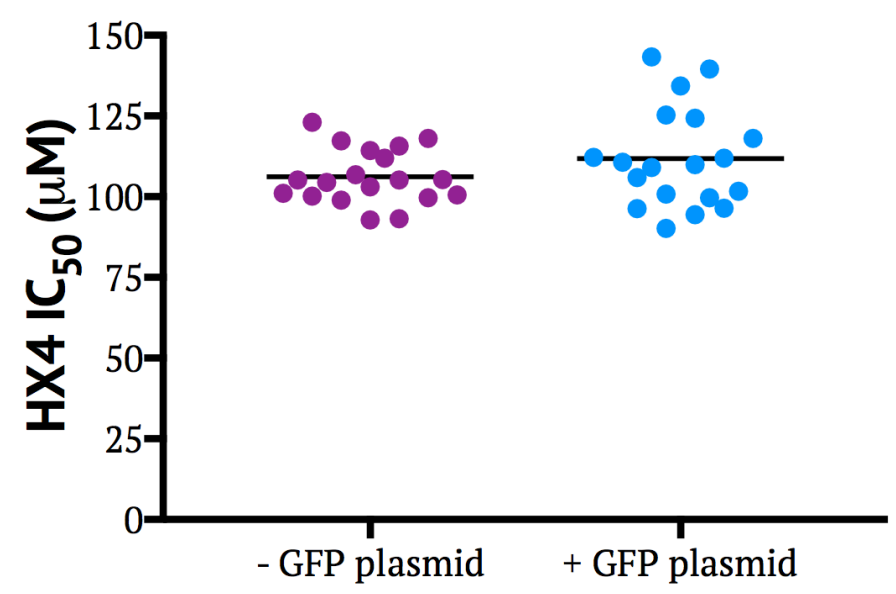

Figure 4-17 IC $C_{50}$ values for HX4 mediated growth inhibition of NfsA_Ec overexpressed in biological replicates of 7NT or SOS-R4 strains. 19 different biological replicates of pUCX:NfSA_Ec in 7NT (-GFP plasmid; purple) and 19 different biological replicates of $p \bar{U}$ CX:NfSA_Ec colonies in SOS-R4 (+GFP plasmid; blue) were subjected to HX4-dependent growth inhibition, which was monitored by measuring strain turbidity $\left(O D_{600}\right)$ pre- and post-four hour incubation with a two-fold dilution series of HX4, with a top concentration of $300 \mu \mathrm{M}$. Percentage growth relative to unchallenged controls was determined and used to calculate the concentration at which 50\% growth inhibition was seen using GraphPad Prism 7.0. Data are the average of 3 biological replicates (error bars omitted for clarity). Differences between the mean IC $C_{50}$ values in $7 N T$ and SOS-R4 colonies were not statistically significant when compared using unpaired Student's t-tests $(p$ value $=0.17)$.

\subsubsection{Attempts to develop unstable GFP reporter strains}

The results from Section 4.3.4 showed that background levels of GFP played a role in the sensitising of $E$. coli cells over-expressing NfsA_Ec to nitroreductase-activated SOS-inducing compounds. We postulated that this background GFP could have been caused by natural cellular induction of the SOS response such as in response to changes in $\mathrm{pH}$ (Dri and Moreau, 1994) or during transition into early stationary phase (Taddei et al., 1995), and were exacerbated by the long half-life of GFPmut3b ( $>24$ hours) (Andersen et al., 1998). We therefore sought to determine if the use of more unstable GFP variants, having half-lives of less than two hours might mitigate this effect, by ensuring substantial levels of GFP were only produced when the SOS 
response was activated in E. coli cells following nitroreductase-activated compound-mediated DNA damage.

\subsubsection{Cloning of unstable GFP variants into pANODuet-1}

One of the natural protein degradation pathways in E. coli involves recognition of prematurely terminated peptides by ssrA mRNA, which leads to the addition of a peptide tag, AANDENYALAA, to the C-terminal end of the protein and degradation by tail-specific proteases (Keiler et al., 1996). For the study of transient gene expression in E. coli, Andersen et al., (2008) constructed GFP variants containing different C-terminal peptide tags to study their effects on the half-life of GFP. Four different variations of this tag were added to the end of the $g f p$ gene sequence, as changes in the last three residues of the tag sequence had been shown to affect the half-life stability of proteins (Keiler and Sauer, 1996). This led to the construction of four different unstable GFP variants (with AANDENYALAA, AANDENYALVA, AANDENYAASV, or AANDENYA $\underline{A A V}$ C-terminal tags), having half-lives ranging from 40 minutes (LVA and LAA), to 60 minutes (ASV) and 110 minutes (AAV) (Andersen et al., 1998).

We utilised similar primers to those designed by Andersen et al. to add these four different tags to the 3' end of the gfpmut3b gene (see Figure 4-18 for Cterminal tag sequences). Andersen et al. also included a six base-pair sequence between the end of the $g f p$ gene and the peptide tags, corresponding to a StuI restriction site, however this was presumed to be unimportant for protease recognition and was omitted from our primer sequences. Our forward primer also included the accidental amino acid change S2R introduced by Andersen et al. during their creation of GFP variants; however they stressed that this did not appear to cause any phenotypic changes in the protein (Andersen et al., 1998). The four different unstable GFP genes were PCR amplified and re-cloned into pANO:Duet-1 in place of GFPmut3b. 


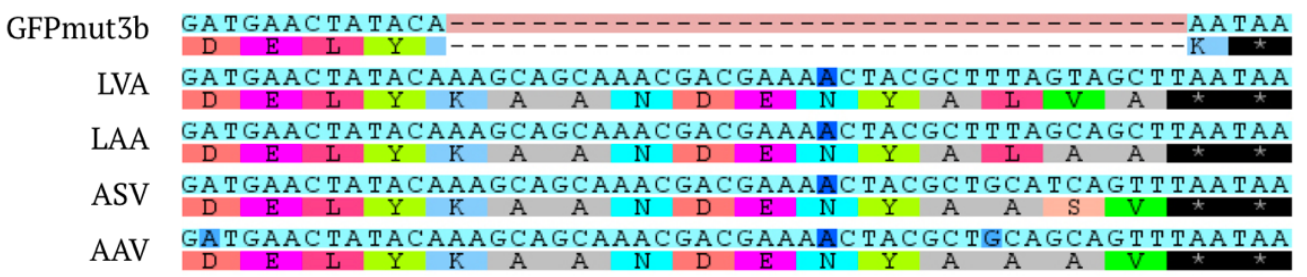

Figure 4-18 Sequence alignment of GFPmut3b and unstable GFP variant genes (including amino acid translations). Alignment shows the final five amino acids in GFPmut3b, and the four different peptide tags added to the C-terminal end of GFPmut3b to generate four different unstable GFP variants.

\subsubsection{Growth inhibition $\left(I C_{50}\right)$ assays with pUCX:NfsA_Ec and GFP unstable variants in 7NT with metronidazole}

To determine if these unstable GFP variants could be substituted for pANODuet-1:GFP in $\mathrm{IC}_{50}$ assays, and whether this would effectively resolve the issue of $E$. coli cells becoming hyper-sensitised to genotoxic prodrug metabolites, the effect of each construct on the growth inhibition of 7NT cells also over-expressing NfsA_Ec following challenge with metronidazole was determined.

7NT cells bearing pUCX:NfsA_Ec were transformed with each of the four pANODuet-1:unstable_GFP plasmids, and 18 different biological replicates from each co-transformation, alongside 18 different biological replicates each of SOS-R4 cells bearing pUCX:NfsA_Ec and 7NT cells containing both pUCX:NfsA_Ec and pCDFDuet-1 (i.e., a GFP-null control for the pANODuet-1 plasmid), were subjected to growth inhibition assays with $50 \mu \mathrm{M}$ metronidazole (Figure 4-19). 


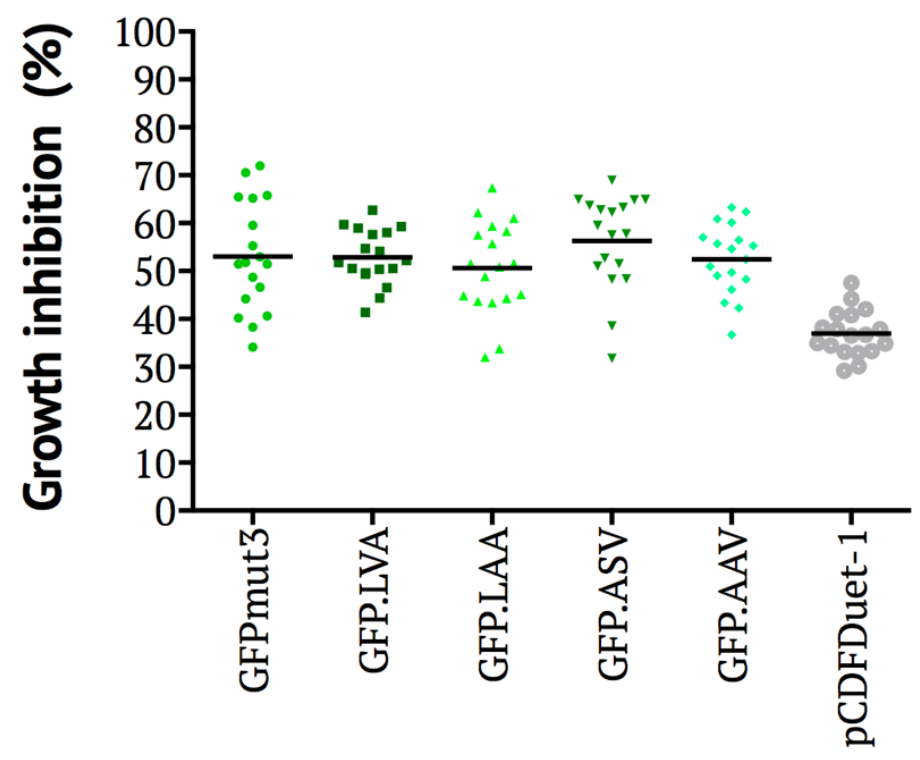

Figure 4-19 Metronidazole mediated growth inhibition of 7NT cells co-expressing pUCX:NfsA_Ec and pANODuet-1:GFP variants. 7NT cells expressing pUCX:Nfs__Ec and also co-expressing either pANODuet-1:GFP (SOS-R4 cells), pANODuet-1:GFP unstable variants (GFP.LVA, GFP.LAA, GFP.ASV, GFP.AAV), or $p C D F D u e t-1$ were subjected to metronidazole dependent growth inhibition, which was monitored by measuring strain turbidity $\left(\mathrm{OD}_{600}\right)$ pre- and post-four hour incubation with $50 \mu \mathrm{M}$ metronidazole. Percentage growth relative to unchallenged controls was determined. Data are the average of 3 biological replicates (error bars omitted for clarity).

The average growth inhibition of 7NT cells co-expressing pUCX:NfsA_Ec and pCDFDuet-1 was 37\% (with 13\% standard deviation). It was reasoned that if 7NT cells co-expressing a particular unstable GFP variant exhibited similar levels of growth inhibition, then that particular fluorescent protein would be unlikely to play a role in the sensitising of E. coli to SOS-inducing nitroaromatic compounds. If so, it could be used to replace GFPmut3b in the pANODuet:1 plasmid.

However the average growth inhibition of 7NT cells bearing any of the four unstable GFP variants was over 50\%, similar to that observed in SOS-R4 cells, indicating that even the presence of less stable forms of GFP still had an effect on sensitising $E$. coli to nitroreductase-mediated growth inhibition. Furthermore, 7NT cells expressing any of the four unstable GFP variants showed widely varying colony sizes when plated on LB agar plates, a 
phenomenon not observed with plated SOS-R4 cells (results not shown), suggesting that the unstable GFP variants affected $E$. coli growth negatively and to a far greater extent than GFPmut3b. As a consequence, efforts to improve the GFP reporter strain for nitroreductase were not pursued further.

\subsection{Discussion}

The research described in this chapter involved the introduction of random mutations into the genes encoding YcnD_Bs and $\mathrm{YfkO}_{-} \mathrm{Bs}$, and selection of improved S33-activating variants using an E. coli GFP-based reporter strain and FACS. Ultimately three YfkO_Bs variants generated after two rounds of random mutagenesis and selection were found to sensitise E. coli to over 2.4fold lower concentrations of S33 than wild-type YfkO_Bs, and purified enzymes of two of these variants exhibited 4-fold and 6.6-fold improvements in $k_{\text {cat }} / K_{M}$ with S33 respectively. When compared to improved NfsA_Ec variants generated in Chapter 3, YfkO variant M2 3_62 only showed a 2-fold lower $k_{\text {cat }} / K_{M}$ than NfsA R225A with S33, but exhibited both a much higher $k_{\text {cat }}$ (8-fold higher) and $K_{M}$ (16-fold higher). As neither variant has yet been transfected into cancer cell lines, it is difficult to determine which kinetic profile may translate to improved activation of S33 in human tissues. The two YfkO M2 variants 3_62 and 1_88 also exhibited the capacity to sensitise E. coli to lower concentrations of next-generation PET imaging probes than YfkO_Bs.

One key limitation of the evolution conducted on YfkO_Bs in this chapter was the inability to generate large error-prone libraries containing $>10^{3}$ variants prior to FACS, and YfkO activity with S33 could likely be improved further with subsequent rounds of mutagenesis and selection. However, the generation of improved NfsB family enzymes for CDEPT clinical trials was a major driver of the work conducted in this chapter, and YfkO M2 variants counter-screened alongside other nitroreductase candidates with the nextgeneration lead candidate prodrug SN36506 showed only moderate levels of activity with this substrate. Ultimately the NfsB_Vv variant F70A/F108Y, 
which was screened in this panel of enzymes, showed the ability to sensitise E. coli to nearly two-fold lower concentrations of SN36506 than the YfkO variants, and we decided to complete our evolution of YfkO_Bs here and move onto attempts to improve NfsA_Ec activity with S33 further in

\section{Chapter 5.}

During PCR amplification of a mixed template of leading Round 1 genes for Round 2 directed evolution, using the GeneMorphII DNA polymerase, we noticed the generation of variants that possessed combinations of mutations found in Round 1 variants. Two of the leading YfkO variants found in Round 2, M2 3_62 and 1_32, only contained amino acid substitutions that had been combined from different Round 1 variants, illustrating the potential of DNA shuffling to reveal the synergistic effects of combined mutations on enzyme activity. The inclusion of multiple template variant genes from lead candidates in epPCR reactions should allow for this approach to enzyme evolution to occur in future directed evolution projects.

Attempts to improve the activity of YcnD_Bs by using site-targeted libraries in Chapter 3 had previously shown limited success, with the best variants showing a 3-fold improvement in $k_{\text {cat }} / K_{M}$ over wild-type YcnD_Bs, which was still nearly 3-fold lower than that observed in the NfsA_Ec variant R225A. Unfortunately attempts to improve YcnD_Bs activity using random mutagenesis and FACS selection were also unsuccessful due to the initial comparisons against a wild-type YcnD_Bs control that expressed at far lower levels, and the nature of which was not discovered until after the two rounds of directed evolution. This meant that improvements in the ability of variants to generate higher levels of S33-induced GFP production over wildtype YcnD_Bs or sensitise E. coli to lower lethal concentrations of S33, had been artificially inflated until the discovery that the parent enzyme was cloned into low-expressing pUCX. Furthermore, experiments conducted in Section 3.3.1.1 indicated that high intracellular levels of over-expressed YcnD_Bs induced by IPTG concentrations over $10 \mu \mathrm{M}$ showed a diminished ability to reduce the 5-nitroimidazole prodrug metronidazole, which we 
have speculated is due to the inability of $E$. coli to generate enough intracellular NADPH to keep up with the reaction. Wild-type YcnD_Bs induced by $50 \mu \mathrm{M}$ IPTG in high-expressing pUCX also exhibits high levels of activity with S33, which could impede efforts to accurately determine any improvements in activity of YcnD variants with the probe in vivo. Thus for any future directed evolution work involving YcnD_Bs, the lowering of concentrations of IPTG to $\leq 10 \mu \mathrm{M}$ should allow for accurate assessment of the ability of YcnD variants to activate S33 in E. coli. Alternatively, metabolic engineering via co-expression of an intracellular cofactorregenerating enzyme such as glucose dehydrogenase (which is currently being investigated in the Ackerley lab in vitro as a means of regenerating NADPH for nitroreductase kinetic assays with metronidazole) might provide a viable means of maintaining concentrated pools of NADPH in the cell. During the creation of error-prone libraries, we experienced issues both in generation of $E$. coli cells with high enough electrocompetent efficiency to transform in large numbers of variants, and with low cloning efficiency of epPCR products into the pUCX plasmid (both these protocols have since been optimised over the course of my $\mathrm{PhD}$ and are detailed in Sections 2.6.6.1 and 2.6.9.3). This severely limited our ability to create large random mutagenesis libraries, which would have limited our potential to find improved S33-activating variants. In particular, no large libraries of YfkO_Bs variants (both YfkO M1 and M2 libraries contained $<10^{4}$ post-niclosamide selection) were ever subjected to FACS. The small library sizes of both YfkO M1 and M2 libraries could have explained our apparent inability to select for variants that induced a $>2$-fold increase in fluorescence over wild-type YfkO_Bs following challenge with S33 post-FACS. The comparison of niclosamide-selected variants directly against those selected by FACS would have revealed if this selection, was in fact not allowing for the recovery of variants most capable of activating S33, and only allowing for the selection of more mediocre YfkO enzymes. 
The discovery that pANODuet-1:GFP was contributing to the sensitising of E. coli to lethal concentrations of nitroaromatic compounds, including S33, came as a surprise as we had not previously observed this in the E. coli SOSR2 strain, which contains a chromosomally integrated lacZ reporter instead of a plasmid-based GFP reporter. We postulate that this is due to toxicity of GFP to the cells, however our attempts to utilise unstable GFP variants in its place did not alleviate this problem, in part due to their irregular effects on cell growth. Use of other coloured fluorescent proteins that are not cytotoxic, such as red fluorescent proteins (Shemiakina et al., 2012), could alleviate this issue, with an added advantage of the longer-wavelength fluorescent reporter proteins being that their excitation/emission wavelengths do not overlap with $E$. coli autofluorescence, and therefore would likely improve single-cell analysis and sorting during flow cytometry experiments.

However in Section 4.3.4 we showed that different SOS-R4 cultures exhibited varying levels of background activation of the SOS response, leading to different basal levels of GFP in cells. This could potentially be due to the lower natural levels of repression of a high-copy number plasmidbased SOS reporter (for which there is still only a single chromosomal copy of the lexA repressor gene). We theorised that this could have limited the efficacy of FACS sorting of the most active variants, as it could allow for both the selection of cells with high background levels of GFP that do not necessarily exhibit the highest activity with S33, as well as catalytically superior variants. To solve this issue, more basal repression of background induction of the $E$. coli SOS response and genes under control of the plasmid-based sfiA' promoter could be generated through increasing the increasing the copy number of the SOS repressor gene lexA. The cloning of lexA into the second multiple-cloning site of pANODuet-1 could potentially allow for high levels of GFP reporter gene production only after prodrug or probe-mediated DNA damage. 
Finally, we observed low recovery of sorted events collected during FACS ( $<20 \%$ of sorted cells). Fluorescent DNA-binding dyes, such as propidium iodide which does not cross intact cell membranes (Jepras et al., 1995), could be utilised in future FACS experiments to allow for more efficient selection of fluorescent cells that are more likely to recover following FACS. Double gating E. coli cells on both intracellular GFP production and propidium iodide binding has been shown to successfully distinguish between populations of live cells, membrane-compromised cells and dead cells (Lehtinen et al., 2004), and selection of non-propidium iodide-binding cells could allow for improved recovery rates of cells post-FACS. 


\section{Chapter 5. Validation of positive selection compounds to improve NfsA_Ec activation of S33}

\section{$5.1 \quad$ Introduction}

The primary strategies described thus far for screening and selection of nitroreductases exhibiting improved abilities to activate S33 have involved specialised E. coli screening strains, which generate quantifiable colorimetric or fluorometric products following induction of an SOS response promoter. However, there have been clear limitations with each approach- the relatively low-throughput LacZ-based screen limits library sizes that can be easily screened to $<10^{4}$ variants, whereas the GFP-based screen, which allows for high-throughput screening using FACS, suffers from a high rate of selection of highly fluorescent variants that do not necessarily exhibit significantly improved activity with S33.

In Chapter 4 low concentrations of the 'anti-prodrug' niclosamide were applied as a mild selection pressure to random mutagenesis libraries, allowing for the elimination of inactive nitroreductases from these libraries prior to more targeted S33-specific screening. In this chapter, we explored the potential of niclosamide and other positive selection compounds for use as sole selection tools to recover improved S33-activating variants from an immense NfsA_Ec multi-site saturation mutagenesis library.

\subsubsection{The NfsA_Ec 7SM library}

The 7SM library was designed as an NfsA_Ec site-targeted library resource for the Ackerley lab, using both empirical and computational data to select seven residues in the active site of NfsA_Ec which appeared amenable to productive amino acid substitutions (Figure 5-1). The empirical data involved previous evolution conducted on NfsA_Ec to improve activity with the prodrug PR-104A (Copp et al., 2017) or with the 2-nitroimidazole PETimaging agent EF5 (Williams, 2013), which identified five active site residues 
where different amino acid substitutions improved enzyme activity with these substrates (S41, F42, F83, K222, R225). The online software HotSpot Wizard 1.7 (http://loschmidt.chemi.muni.cz/hotspotwizard/; Pavelka et al., 2009) was then used to computationally identify two other catalytically important sites that showed high amino acid diversity over databasedeposited gene sequences (S224, F227).

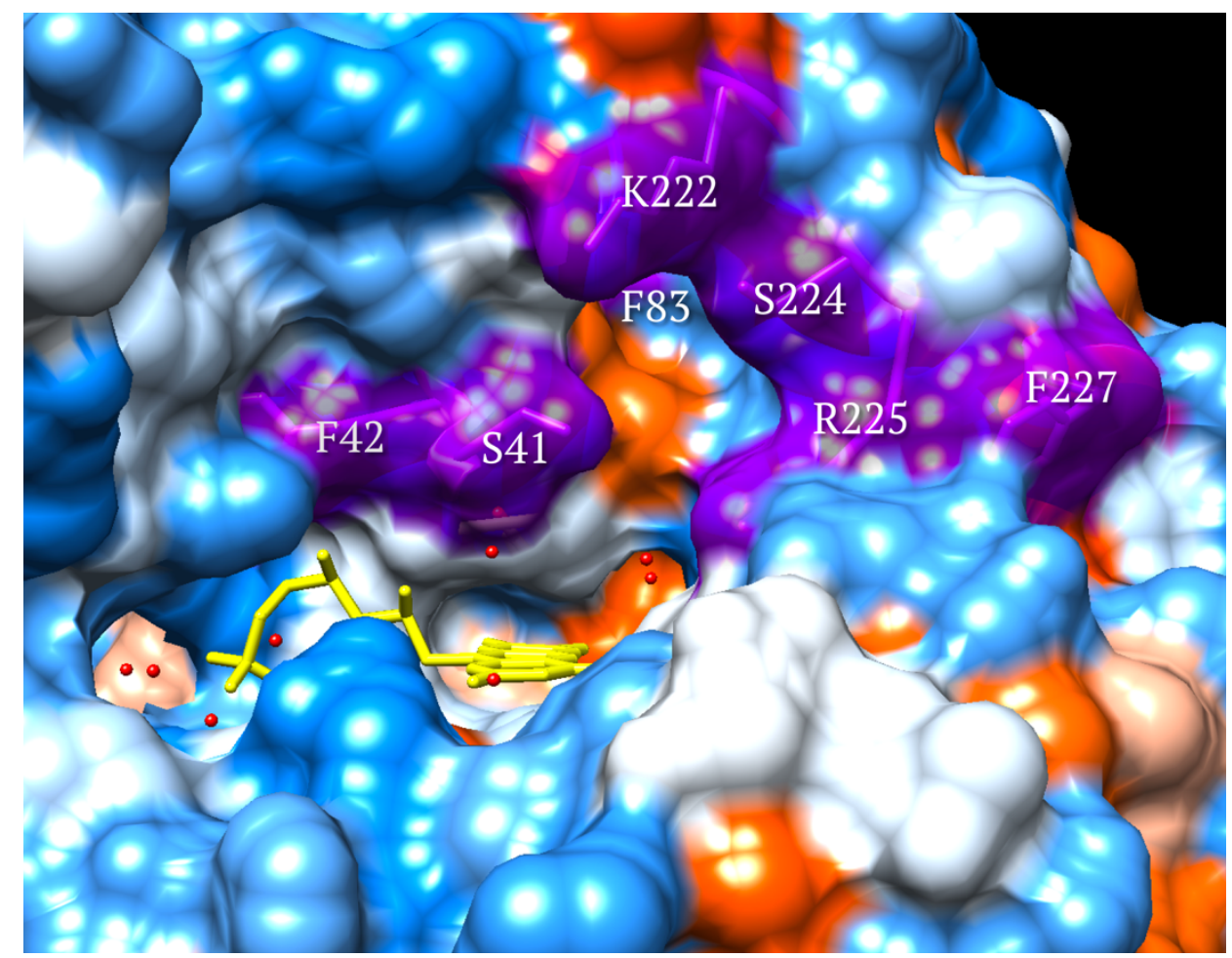

Figure 5-1 Active site residues of NfsA_Ec targeted for site saturation mutagenesis in the 7SM library. Modelling of active site residues in NfSA_Ec that are mutated in the 7SM library, highlighted in purple. The FMN molecule is coloured yellow.

To introduce alternative amino acids at six of the target positions, the corresponding codon in the $n f_{s} A$ gene was mutated using an NDT degeneracy (N: A/C/T/G; D: A/G/T, T: T; Table 5-1). An NDT degenerate codon allows for 12 possible codons that specify 12 unique amino acids (Phe, Leu, Ile, Val, Tyr, His, Asn, Asp, Cys, Arg, Ser, Gly) - a balanced mix of polar and non-polar, alipathic and aromatic, and negatively and positively charged amino acids (Reetz et al., 2008); it also allowed for the wild-type 
amino acid to be encoded at each of these sites. For amino acid site 222, as the wild-type Lys could not be coded for with NDT, the degenerate codon NNK (K: guanine/thymine; Table 5-1), was used instead, allowing for 32 codons and all 20 proteinogenic amino acids to be encoded (Reetz et al., 2008). The 7SM library had in total nearly 96 million possible codon combinations, and was ordered as a synthetic gene cloned into what was discovered to be low-expressing pUCX (see Section 3.3.1); consequently, all assays in this chapter were performed in comparison to a positive control of wild-type NfsA_Ec in low-expressing pUCX.

\begin{tabular}{cc}
$\begin{array}{c}\text { WT aa } \\
\text { residue }\end{array}$ & $\begin{array}{c}\text { NDT/NNK codon } \\
\text { degeneracy }\end{array}$ \\
\hline S41 & NDT \\
F42 & NDT \\
F83 & NDT \\
K222 & NNK \\
S224 & NDT \\
R225 & NDT \\
\hline F227 & NDT \\
\hline
\end{tabular}

Table 5-1 NfsA_Ec amino acid residues targeted for site saturation mutagenesis in the 7SM library with degenerate codons.

At this stage of my research the 7SM library was (by several orders of magnitude) the largest site-targeted nitroreductase variant library the Ackerley group had designed (the prior largest libraries created by our group each only containing approximately 1700 variants; Copp et al., 2017; Swe et al., 2012). Owing to the drastic randomisation of the NfsA active site, it was anticipated to provide a large collection of variants of which the majority would be non-functional; however it was reasoned that the potential for selection of functional variants possessing very different active sites to wildtype NfsA_Ec could allow for rapid improvement of activity with non-natural substrates. 


\subsubsection{Chloramphenicol as a positive selection compound}<smiles>O=C(NC(CO)C(O)c1ccc([N+](=O)[O-])cc1)C(Cl)Cl</smiles>

Figure 5-2 Chemical structure of chloramphenicol.

Chloramphenicol (chemical structure shown in Figure 5-2), one of the few naturally occurring nitro-compounds, is produced by different bacterial species including Streptomyces venezuelae (Fernández-Martínez et al., 2014), and was the first broad-spectrum antibiotic introduced to the market in 1949 (Feder et al., 1981). It is active against a wide range of Gram-positive and Gram-negative bacteria, and works through inhibition of the bacterial ribosome (Feder et al., 1981). Although unknown to us at the time, nitroreduction of chloramphenicol to the amine product has previously been hypothesised as a possible mechanism for resistance to chloramphenicol in bacterial species (Yunis, 1988). Preliminary research conducted by our lab had indicated that it could have potential use as a positive selection tool for nitroreductases in the same manner as niclosamide (Dr Elsie Williams, personal communication).

\subsubsection{Counter-screening of selected NfsA_Ec variants with nitroaromatic compounds}

The primary goal of the research described in this chapter was to discover NfsA_Ec variants that showed improved capabilities to activate S33; however, to further investigate the potential of niclosamide or chloramphenicol selection(s) for variants exhibiting a range of desirable activities, selected variants were also counter-screened with other nitroaromatic compounds of potential interest, as detailed below. 


\subsubsection{CB1954}

As described in Section 1.4, there has long been interest in the development of improved nitroreductases for the activation of CB1954 in nitroreductasebased GDEPT, in particular enzymes that could efficiently activate this prodrug at concentrations below that of the maximum tolerated dose in humans (ca. $5.8 \mu \mathrm{M}$; Chung-Faye et al., 2001). There has also been interest in our research group in the evolution of nitroreductases for improved activation of the next-generation prodrug PR-104A (Section 1.4.3, e.g., Copp et al., 2017); however, due to limited stocks of this propriety compound, in this current study 7SM variants were only conducted with CB1954.

\subsubsection{Metronidazole and zebrafish targeted cell ablation}

The zebrafish is an excellent vertebrate disease model organism for investigating development from the transparent embryonic stage onwards, and it exhibits both phenotypic and genetic similarities to humans, which allows for the modelling the role of specific cells or tissues in human development or physiological processes and for studying tissue regeneration (Lieschke and Currie, 2007). One method developed for conditional ablation of target zebrafish cells involves the combination of $E$. coli NfsB and the clinically relevant 5-nitroimidazole antibiotic metronidazole (chemical structure shown alongside that of S33 in Figure 5-3), with target cells being defined via $n f s B$ expression under control of a tissue-specific promoter in a transgenic zebrafish line (Curado et al., 2007; Pisharath et al., 2007).

Activation of metronidazole by NfsB converts it from an inactive prodrug into a DNA interstrand cross-linking agent (Bridgewater et al., 1997; Edwards, 1993). However, unlike CB1954, the products formed from metronidazole reduction are not cell-permeable and do not generate a bystander effect (Bridgewater et al., 1997), which makes it an ideal drug for targeted ablation studies as the active metabolites are confined to the target cell. 
<smiles>Cc1ncc([N+](=O)[O-])n1CC(=O)NCC(F)(F)F</smiles>

S33<smiles>Cc1ncc([N+](=O)[O-])n1CCO</smiles>

Metronidazole

Figure 5-3 Chemical structure of the 5-nitroimidazole compounds metronidazole and $S 33$.

Unfortunately, this system suffers from the inherent poor activity of $E$. coli $\mathrm{NfsB}$ with metronidazole, which necessitates lengthy co-incubation of zebrafish with the prodrug (>24 h), and a challenge concentration $(10 \mathrm{mM})$ that is close to lethal levels of metronidazole for zebrafish (Curado et al., 2007). As well the potential for NfsA_Ec 7SM variants that show improved activity with metronidazole to be used as alternative enzymes in zebrafish targeted ablation, we sought to investigate its ability to act as a potential surrogate for preliminary screening of 7SM variants for improved S33 activation, due to our limited stocks of the latter. Both are 5-nitroimidazole compounds, and hence share identical structures at the nitroaromatic ring, where reduction by the nitroreductase occurs (Figure 5-3).

\subsubsection{2-nitroimidazole compounds}<smiles>O=[N+]([O-])c1nccn1CC(O)CNCCBr</smiles>

RB6145<smiles>O=[N+]([O-])c1nccn1CC(O)CN1CC1</smiles>

RSU1069<smiles>O=[N+]([O-])c1ncc[nH]1</smiles>

Azomycin

Figure 5-4 Chemical structures of 2-nitroimidazole compounds RB6145, RSU1069, and azomycin. 
As described in Section 1.6.4, previous research conducted in the Ackerley lab evaluated nitroreductases for their ability to active 2-nitroimidazole hypoxia-activated PET imaging probes, such as EF5, F-MISO and HX4 to repurpose them for clinical imaging of nitroreductase localisation during GDEPT (Williams, 2013). However, due to limited stocks of all three probes, variants were instead preliminarily screened for activity with the 2nitroimidole drug RB6145 (the precursor to RSU1069, a hypoxic cell radiosensitiser and chemotherapeutic; Jenkins et al., 1990; chemical structures of compounds shown in Figure 5-4; Williams, 2013). Unfortunately, our stocks of RB6145 were subsequently limited, and for the purposes of the research conducted in this chapter, we turned to azomycin (chemical structure shown in Figure 5-4) as an alternative, more readily available 2-nitroimidazole surrogate for EF5 and HX4 activity.

\subsubsection{Nitrofurans}<smiles>O=C1CN(/N=C/c2ccc([N+](=O)[O-])o2)C(=O)N1</smiles>

Nitrofurantoin<smiles>NC(=O)N/N=C/c1ccc([N+](=O)[O-])o1</smiles>

Nitrofurazone

Figure 5-5 Chemical structures of the nitrofuran drugs nitrofurantoin and nitrofurazone.

Nitrofurazone and nitrofurantoin (chemical structures shown in Figure 5-5) are two nitrofuran drugs used clinically for the treatment of bacterial infections (Guay, 2001). Both E. coli NfsA and NfsB enzymes are capable of activating nitrofurazone, with mutations in $n f s A$ (first-step inactivation) followed by $n f_{s} B$ (second-step inactivation) genes found in nitrofurazoneresistant E. coli strains (Bryant et al., 1981; Whiteway et al., 1998). Other nitroreductases including NfrA from B. subtilis (Zenno et al., 1998), NfsA 
from Enterobacter cloacae (Bryant and DeLuca, 1991), Frp from V. harveri (Kwak et al., 2003), NprA from Rhodobacter capsulatus (Pérez-Reinado et al., 2008), SnrA and Cnr from S. typhimurium (Salamanca-Pinzón et al., 2006), and FrxA and RdxA from H. pylori (Olekhnovich et al., 2009; Sisson et al., 2002) all exhibit the ability to activate nitrofuran drugs. Consistent with this, the Ackerley lab has found that the majority of NfsA and NfsB family enzymes from the 58 nitroreductase library are able to reduce these compounds (Condon, 2013). To better probe the activity range of selected nitroreductases with structurally diverse nitroaromatic substrates, these compounds were included in the panel of substrates to be counter-screened with NfsA_Ec 7SM variants.

\subsubsection{Bioremediation of nitrotoluenes}

2,4,6-trinitrotoluene (TNT) has been heavily produced as an explosive and is a prevalent and toxic environmental pollutant. Residual TNT and its manufacturing by-products, including 2,4-dinitrotoluene (2,4-DNT; both chemical structures shown in Figure 5-6), have been found near manufacturing plants and above land mines (Frische, 2002; Jenkins et al., 2001; Neuwoehner et al., 2007; Sylvia et al., 2000).<smiles>Cc1c([N+](=O)[O-])cc([N+](=O)[O-])cc1[N+](=O)[O-]</smiles>

TNT<smiles>Cc1ccc([N+](=O)[O-])cc1[N+](=O)[O-]</smiles>

2,4-DNT

Figure 5-6 Chemical structures of TNT (2,4,6-trinitrotoluene) and 2,4-DNT (2,4dinitrotoluene)

Type I nitroreductases from Klebsiella (Kim and Song, 2005; Kim et al., 2002), Pseudomonas (Caballero et al., 2005), Vibrio (Kwak et al., 2003), 
Gluconobacter (Yang et al., 2016), Escherichia (González-Pérez et al., 2007; Yin et al., 2005) and Clostridium species (Kutty and Bennett, 2005) have been shown to be able to reduce the nitro groups of TNT and/or 2,4-DNT to generate hydroxylamine products for use as sources of nitrogen for host organism growth (González-Pérez et al., 2007). Mutation of E. coli NfsB showed that the single amino acid substitutions F123A and F124W, and the double variant F123A/F124W all exhibited improved $k_{c a t} / K_{M}$ with TNT over wild-type NfsB (Bai et al., 2015b). There has also been interest in the use of transgenic tobacco plants expressing nitroreductases for the biodegradation of TNT (Hannink et al., 2001; Kurumata et al., 2005; Van Dillewijn et al., 2008). Previous research conducted in the Ackerley lab showed that NfsA (but not NfsB) family enzymes showed the capacity to active 2,4-DNT (Williams, 2013).

\subsection{Aims}

- Evaluate the potential of azomycin and metronidazole to act as screening surrogates for the 2-nitroimidazole RB6145 and 5nitroimidazole S33, respectively.

- Use niclosamide and chloramphenicol as positive selection compounds to recover functional nitroreductase variants from a heavily active-site-randomised library of $n f s A$ variants.

- Screen variants selected from the second aim for activity with a range of nitroaromatic compounds.

\section{$5.3 \quad \underline{\text { Results }}$}

\subsubsection{Evaluation of metronidazole and azomycin as surrogate compounds for RB6145 and S33}

As described in Sections 5.1.3.2 and 5.1.3.3, due to limited stocks of the compounds S33 and RB6145, we turned to the use of metronidazole or azomycin as surrogates respectively in preliminary screening for improved 
$N f_{S} A$ variants. To evaluate their suitability as surrogates, we compared the abilities of nitroreductases from the 58 nitroreductase library to sensitise $E$. coli 7NT cells to lethal concentrations of all four compounds.

\subsubsection{Correlation of $S 33$ and metronidazole growth inhibition data}

The abilities of 18 different wild-type nitroreductases to sensitise E. coli 7NT cells to lethal concentrations of metronidazole were determined (Table 5-2), and compared to previously calculated $\mathrm{IC}_{50}$ values for the same nitroreductases with S33 (Column 3 of Table 5-2, data duplicated from Table 3-4).

\begin{tabular}{c|c|c|c}
\multicolumn{1}{c}{ Family } & \multicolumn{1}{c}{$\begin{array}{c}\text { Enzyme } \\
(\boldsymbol{\mu M})\end{array}$} & $\begin{array}{c}\text { S33 IC } \\
\mathbf{I C}_{50}(\boldsymbol{\mu M})\end{array}$ \\
\hline NfsA & YcnD_Bs & $19.4 \pm 1.5$ & $4.6 \pm 1.2$ \\
NfsA & CO-Frp_Vh & $21.0 \pm 4.7$ & $23.8 \pm 3.6$ \\
NfsA & NfsA_Vf & $22.2 \pm 1.5$ & $20.7 \pm 5.2$ \\
NfsA & NfsA_Vv & $26.8 \pm 4.5$ & $57.2 \pm 2.0$ \\
NfsB & NfsB_Es & $33.3 \pm 1.3$ & $38.8 \pm 2.2$ \\
NfsA & NfrA_Bs & $35.9 \pm 3.0$ & $43.4 \pm 2.5$ \\
NfsB & FRaseI_Vf & $36.8 \pm 3.7$ & $34.2 \pm 0.5$ \\
NfsA & NfsA_Ec & $40.0 \pm 1.3$ & $105 \pm 7$ \\
NfsA & NfsA_St & $43.6 \pm 3.0$ & $144 \pm 3$ \\
NfsA & NfsA_Ck & $44.9 \pm 3.7$ & $201 \pm 18$ \\
NfsA & NfsA_Kp & $50.5 \pm 8.3$ & $302 \pm 87$ \\
NfsB & YfkO_Bs & $52.1 \pm 6.7$ & $212 \pm 2$ \\
NfsB & NfsB_Vh & $64.3 \pm 9.3$ & $11.7 \pm 1.7$ \\
Nitroreductase 1 & YdgI_Bs & $64.6 \pm 9.5$ & $125 \pm 11$ \\
NfsA & NfsA_Li & $119 \pm 11$ & $326 \pm 60$ \\
NfsA & Frp_Vh & $213 \pm 19$ & $>1000$ \\
NfsA & NfsA_Lw & $265 \pm 30$ & $764 \pm 52$ \\
NfsA & EcD_Pp & $279 \pm 26$ & $411 \pm 104$ \\
& Empty pUCX & $>>300$ & $>>1000$
\end{tabular}

Table 5-2 IC 50 values for S33 or metronidazole mediated growth inhibition of 18 highly active nitroreductases from the 7NT E. coli 58 nitroreductase over-

expression library. Metronidazole-dependent growth inhibition was monitored by measuring strain turbidity $\left(O D_{600}\right)$ pre- and post-four hour incubation across a two-fold dilution series of metronidazole, using a top concentration of $1 \mathrm{mM}$ metronidazole. Percentage growth relative to unchallenged controls was determined and used to calculate the concentration at which 50\% growth inhibition was seen using GraphPad Prism 7.0. Data are the average of 3 biological replicates $\pm S D$. Enzymes are ranked by their ability to sensitise $7 N T$ cells to S33 (data duplicated from Table 3-4). 
With the exception of Frp_Vh, IC 50 values for 7NT cells over-expressing selected wild-type nitroreductases were generated using a top concentration of $1 \mathrm{mM}$ metronidazole. NfsA_Ec induced a two-fold lower $\mathrm{IC}_{50}$ in $7 \mathrm{NT}$ cells with metronidazole than that observed with the enzyme and S33. A log-log correlation was conducted with S33 IC 50 values generated in Section 3.3.2.2 (Figure 5-7). We observed a moderate correlation $\left(\mathrm{R}^{2}=0.57\right)$ between S33and metronidazole-mediated $\mathrm{IC}_{50}$ values in $7 \mathrm{NT}$ strains over-expressing wild-type nitroreductases, and proceeded with the use of metronidazole for the preliminary assessment of the abilities of variants from the NfsA_Ec 7SM library to activate S33.

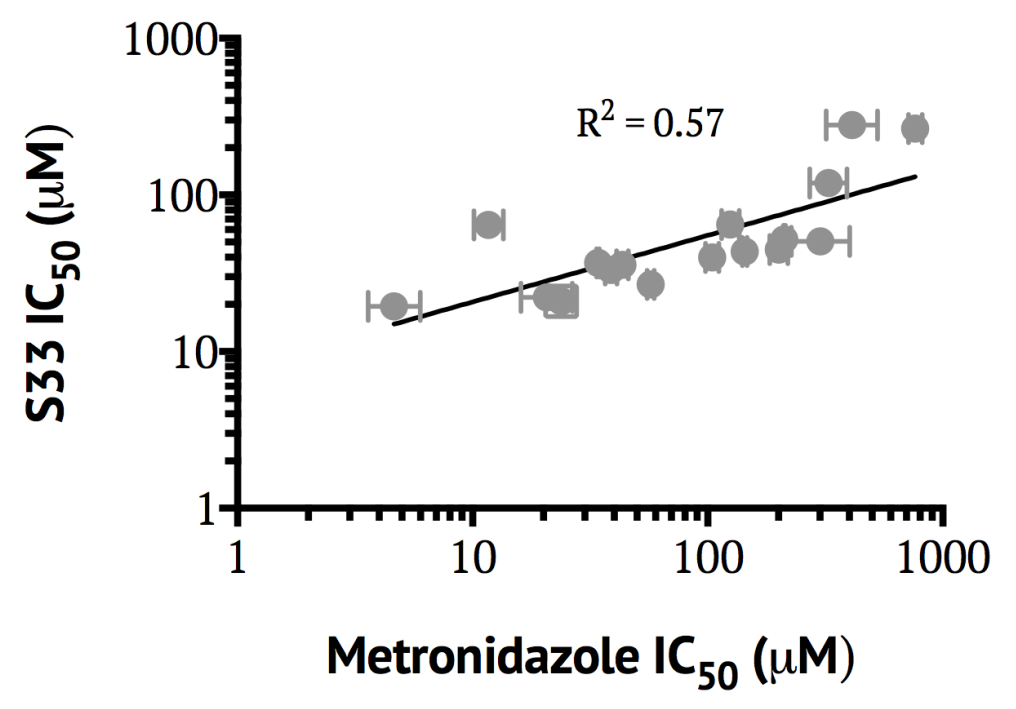

Figure 5-7 Log-log correlation between IC 50 growth inhibitory effects of S33 and metronidazole in $7 N T$ nitroreductase over-expression strains. IC $C_{50}$ values of $7 N T$ cells over-expressing 18 different wild-type nitroreductases from the 58 nitroreductase family following challenge with up to $300 \mu$ M S33 (Table 3-4) and $1000 \mu \mathrm{M}$ metronidazole in IC ${ }_{50}$ assays (Table 5-2). Frp_Vh was dropped from consideration due to its inability to sensitise 7NT cells to up to $1 \mathrm{mM}$ metronidazole.

\subsubsection{Correlation of RB6145 and azomycin growth inhibition}

The abilities of the same 18 different wild-type nitroreductases from Table 5-2 to sensitise E. coli 7NT cells to lethal concentrations of RB6145 or azomycin were also determined (Table 5-3). With the exception of NfsB_Vh, 
$\mathrm{IC}_{50}$ values were able to be generated for $7 \mathrm{NT}$ cells over-expressing selected nitroreductases using a top concentration of $250 \mu \mathrm{M}$ for both RB6145 and azomycin. As previously shown by the Ackerley group, NfsA family enzymes demonstrated higher levels of activity with RB6145 than NfsB family enzymes (Williams, 2013); this trend also held true with azomycin as a substrate. The $\mathrm{IC}_{50}$ value of $7 \mathrm{NT}$ cells over-expressing NfsA_Ec in response to challenge with azomycin was 1.5 -fold higher than that of RB6145. For all NfsB family enzymes analysed, much higher $\mathrm{IC}_{50}$ values were observed with RB6145 than those seen with azomycin. We theorised this could perhaps be due to the smaller size of the latter compound allowing it easier access into the NfsB enzyme active site. This trend was not seen in NfsA family enzymes, which could reflect structural differences in the NfsA and NfsB enzyme active sites - the active site of $E$. coli NfsA is regarded as being more 'open' than that of E. coli NfsB (Vass et al., 2009). A log-log correlation was conducted between RB6145 and azomycin-induced IC 50 values (Figure 5-8). 


\begin{tabular}{|c|c|c|c|}
\hline Family & Enzyme & $\begin{array}{l}\text { RB6145 } \\
I_{50}(\mu \mathrm{M}) \\
\end{array}$ & $\begin{array}{c}\text { Azomycin } \mathrm{IC}_{50} \\
(\mu \mathrm{M})\end{array}$ \\
\hline NfsA & NfsA_St & $6.4 \pm 0.2$ & $10.2 \pm 0.7$ \\
\hline NfsA & NfsA_Ck & $8.9 \pm 1.2$ & $13.5 \pm 2.1$ \\
\hline NfsA & NfsA_Ec & $10.3 \pm 1.6$ & $16.6 \pm 3.3$ \\
\hline NfsA & CO-Frp_Vh & $11.6 \pm 1.2$ & $12.2 \pm 0.7$ \\
\hline NfsA & Frp_Vh & $12.6 \pm 0.5$ & $25.5 \pm 3.9$ \\
\hline NfsA & NfsA_Kp & $13.9 \pm 0.5$ & $19.8 \pm 1.3$ \\
\hline NfsA & EcD_Pp & $15.2 \pm 1.4$ & $24.8 \pm 2.0$ \\
\hline NfsA & NfsA_Vf & $16.5 \pm 1.8$ & $17.6 \pm 1.8$ \\
\hline NfsA & NfsA_Vv & $17.7 \pm 3.1$ & $15.6 \pm 2.0$ \\
\hline NfsA & NfrA_Bs & $17.9 \pm 2.3$ & $13.1 \pm 1.3$ \\
\hline NfsA & YcnD_Bs & $18.7 \pm 4.9$ & $14.5 \pm 1.4$ \\
\hline NfsA & NfsA_Li & $28.9 \pm 5.4$ & $27.8 \pm 1.9$ \\
\hline NfsA & NfsA_Lw & $29.3 \pm 3.3$ & $25.3 \pm 3.4$ \\
\hline Nitroreductase 1 & YdgI_Bs & $41.3 \pm 3.4$ & $51.2 \pm 8.6$ \\
\hline NfsB & FRaseI_Vf & $42.7 \pm 0.5$ & $27.9 \pm 1.2$ \\
\hline NfsB & YfkO_Bs & $109 \pm 11$ & $43.9 \pm 0.5$ \\
\hline NfsB & NfsB_Es & $163 \pm 8$ & $47.4 \pm 6.7$ \\
\hline \multirow[t]{2}{*}{ NfsB } & NfsB_Vh & $>250$ & $50.1 \pm 9.6$ \\
\hline & Empty pUCX & $>>250$ & $>>250$ \\
\hline
\end{tabular}

Table 5-3 IC 50 values for RB6145 or azomycin mediated growth inhibition of 18 nitroreductases from the 7NT E. coli nitroreductase over-expression library. RB6145- or azomycin-dependent growth inhibition was monitored by measuring strain turbidity $\left(O D_{600}\right)$ pre- and post-four hour incubation across a two-fold dilution series of RB6145 or azomycin, with a top concentration of $250 \mu \mathrm{M}$. Percentage growth relative to unchallenged controls was determined and used to calculate the concentration at which 50\% growth inhibition was seen using GraphPad Prism 7.0. Data are the average of 3 biological replicates $\pm S D$. Enzymes are ranked by their ability to sensitise $7 N T$ cells to RB6145.

A moderate correlation $\left(\mathrm{R}^{2}=0.61\right)$ between $\mathrm{RB} 6145$ - and azomycin-mediated growth inhibition in 7NT strains was observed, and on this basis we decided to proceed with azomycin as a surrogate for preliminary determination of the abilities of variants from the NfsA_Ec 7SM library to activate other 2nitroimidazole substrates. 


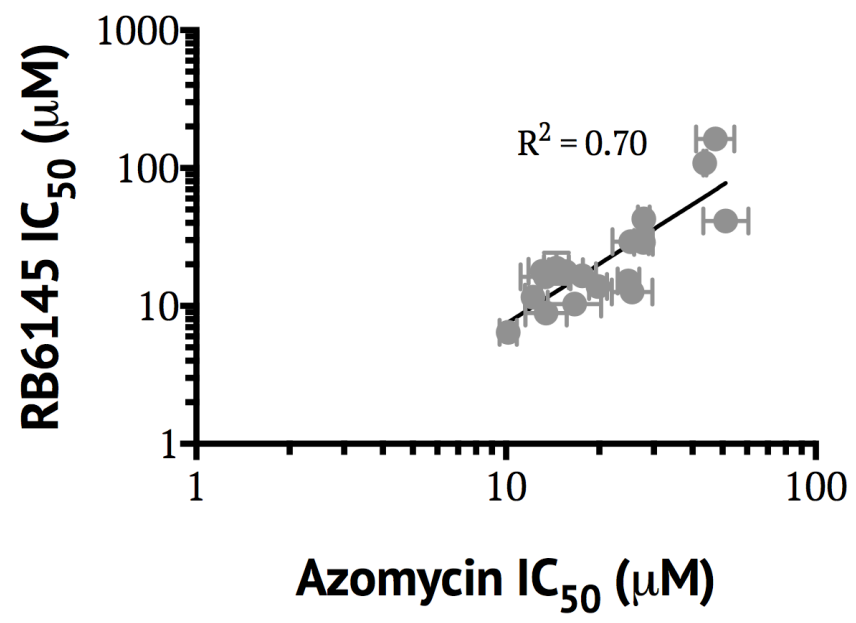

Figure 5-8 Log-log correlation between $I C_{50}$ growth inhibitory effects of RB6145 and azomycin in $7 N T$ nitroreductase over-expression strains. $I C_{50}$ values of $7 N T$ cells over-expressing 18 different wild-type nitroreductases from the 58 nitroreductase library following challenge with up to $250 \mu \mathrm{M}$ RB6145 or $250 \mu \mathrm{M}$ azomycin in $I_{50}$ assays (Table 5-3). $N f_{S} B_{-}$Vh was dropped from consideration due to its inability to sensitise 7NT cells to up to $250 \mu \mathrm{M}$ RB6145.

\subsubsection{Effects of niclosamide selection on 7NT cells over-expressing NfsA_Ec and 7SM library variants}

\subsubsection{Niclosamide selection of 7NT cells over-expressing wild-type NfsA_Ec in low-expressing $p U C X$}

Niclosamide selection of the 7SM library was conducted using two different concentrations of the compound, due to our uncertainty as to how selective niclosamide would be for variants exhibiting improved activity with S33. The plating of 7NT cells containing an empty pUCX plasmid on LB agar plates containing $50 \mu \mathrm{M}$ IPTG and $0.5 \mu \mathrm{M}$ niclosamide led to total cell death (note: $50 \mu \mathrm{M}$ IPTG was used for induction of nitroreductase expression in all LB agar plate selections in this chapter; for clarity this detail will henceforth be omitted). Conversely, we observed full growth of 7NT cells bearing NfsA_Ec in low-expressing pUCX on LB agar plates containing $0.5 \mu \mathrm{M}$ niclosamide, and chose this as our minimum concentration of niclosamide for selection of the 7SM library. When niclosamide was increased to $5 \mu \mathrm{M}$ in LB agar plates, neither the empty plasmid control nor the 7NT cells containing NfsA_Ec in low-expressing pUCX were able to grow effectively $(<1 \%$ of NfsA_Ec- 
expressing cells giving rise to colonies; data not shown), and this was selected as a second concentration for 7SM library selection. With this higher concentration of niclosamide we sought to investigate how the selection of 7SM variants that exhibited higher levels of activity with niclosamide than wild-type NfsA_Ec would impact on their subsequent activities with S33 and other nitroaromatic compounds.

\subsubsection{Niclosamide selection of 7NT cells over-expressing NfsA_Ec 7SM library variants}

Of the 96 million possible variants in the 7SM library, a subset of 11 million variants was transformed into 7NT cells, as we presumed this would allow for enough variability to reduce the possibility of selecting multiple colonies containing the same variant. The plating of these variants on $0.5 \mu \mathrm{M}$ niclosamide was found to select for $4 \%$ of the library, whereas $5 \mu \mathrm{M}$ niclosamide selected for $0.5 \%$ of variants. To determine whether the different levels of niclosamide selection were exerting a discernible influence on the activities of the resulting variants with other nitroaromatic compounds, 57 variants were randomly selected from a plate containing 0.5 $\mu \mathrm{M}$ niclosamide, 57 from a plate containing $5 \mu \mathrm{M}$ niclosamide, and 57 variants from a control plate containing no niclosamide.

We have previously observed varying levels of expression of different nitroreductase candidate genes cloned into high-expressing pUCX and expressed in E. coli (Prosser et al., 2013), and wanted to confirm prior to subsequent activity screening that expression of variants of the 7SM library in $E$. coli 7NT cells was not appreciably different to expression of wild-type NfsA_Ec in low-expressing pUCX. We therefore compared the expression of ten NfsA_Ec 7SM variants from each condition (no niclosamide selection, 0.5 $\mu \mathrm{M}$ niclosamide and $5 \mu \mathrm{M}$ niclosamide) to wild-type NfsA_Ec in lowexpressing pUCX to determine if any of these variants showed appreciable differences in protein expression (Figure 5-9). 
A

MW

(kDa)

35

25

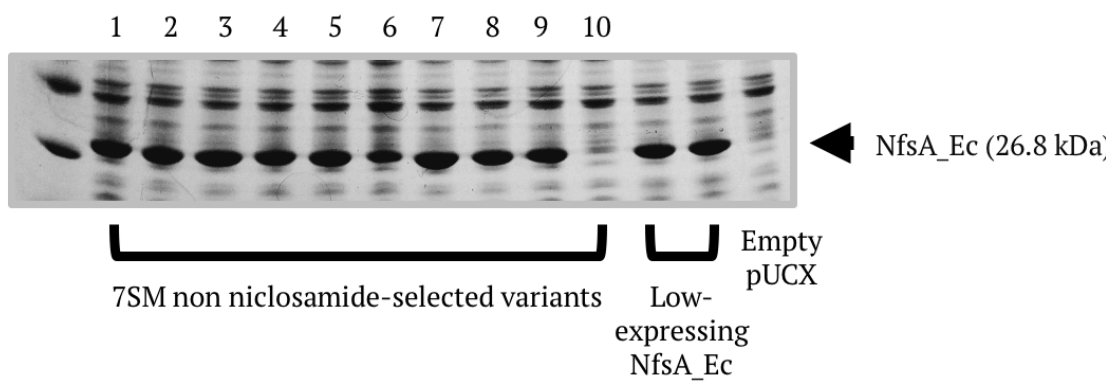

B

\section{MW}

(kDa)

35

25

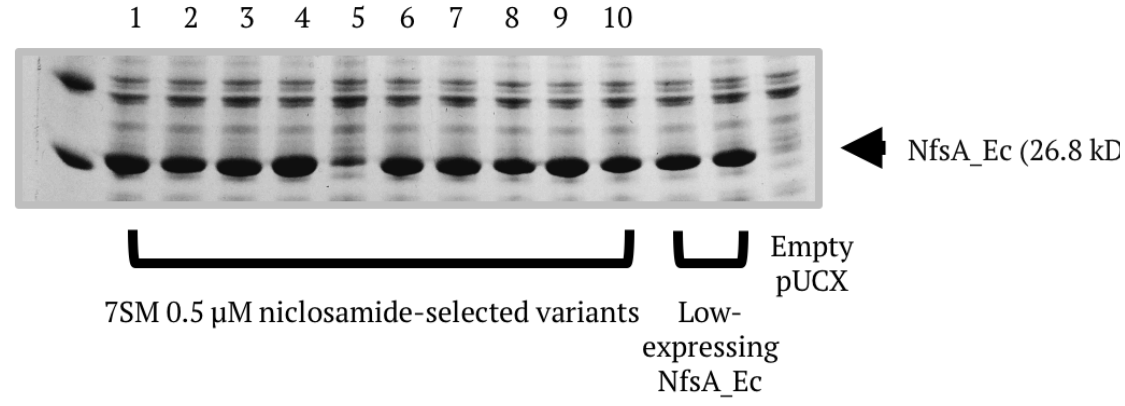

C

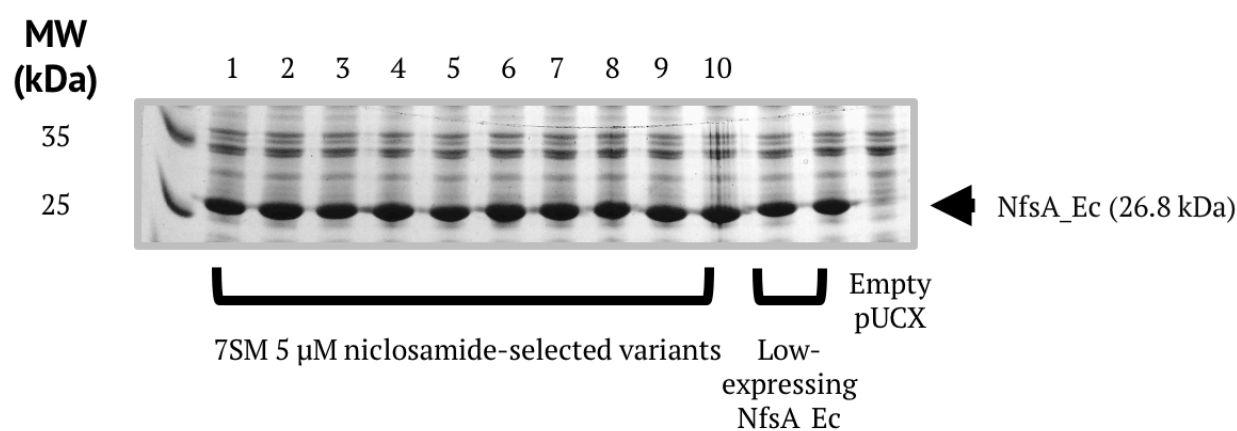

Figure 5-9 SDS-PAGE showing relative protein expression levels of NfSA_EC 7SM library variants and NfSA_Ec in low-expressing pUCX in the E. coli 7NT strain. Relative expression levels of ten NfSA_Ec $7 S M$ library variants chosen from LB agar lacking niclosamide. B) Relative expression levels of ten NfSA_Ec 7SM library variants selected on $0.5 \mu M$ niclosamide. C) Relative expression levels of ten NfsA_Ec 7SM library variants selected on $5 \mu M$ niclosamide. Nitroreductase over-expression was induced with $50 \mu M$ IPTG for four hours prior to sample collection. Samples were normalised for cell density $\left(O D_{600}\right)$ and $10 \mu \mathrm{L}$ of sample loaded per lane.

Of the ten 7SM variants that had not been selected on niclosamide, two showed lower levels of expression than wild-type NfsA_Ec (Figure 5-9A, Lanes 6 and 10), presumably due to the introduction of amino acid substitutions that affected either enzyme stability or functionality. For 
variants that had been selected on $0.5 \mu \mathrm{M}$ niclosamide, one variant exhibited slightly lower levels of expression (Figure 5-9B, Lane 5), and no appreciable differences in expression were observed in any of the ten variants selected on $5 \mu \mathrm{M}$ niclosamide (Figure 5-9C), suggesting that higher concentrations of niclosamide only selected for stable and functional 7SM variants.

\subsubsection{Effects of niclosamide selection on activity of NfSA_EC 7SM variants with other nitroaromatic compounds}

Evaluation of the activities of NfsA_Ec 7SM variants with other nitroaromatic compounds was conducted in the E. coli 7NT strain using growth inhibition assays. 7NT cells bearing different 7SM variants were exposed to a single high concentration of compound that in preliminary assays reduced growth of a culture of 7NT cells containing NfsA_Ec in lowexpressing pUCX to 50\% that of an unchallenged control. Each collection of 57 variants was screened in the inner 60 wells of a single 96 -well plate alongside two 7NT over-expressing NfsA_Ec controls and an empty 7NT pUCX control (Figure 5-10). 
A

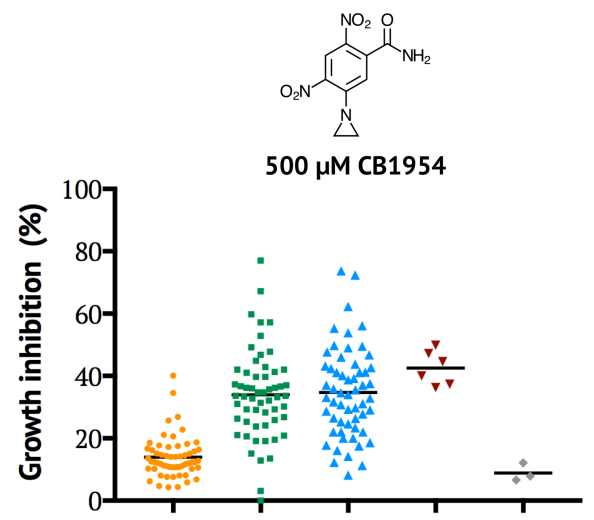

B<smiles>NC(=O)N/N=C/c1ccc([N+](=O)[O-])o1</smiles>

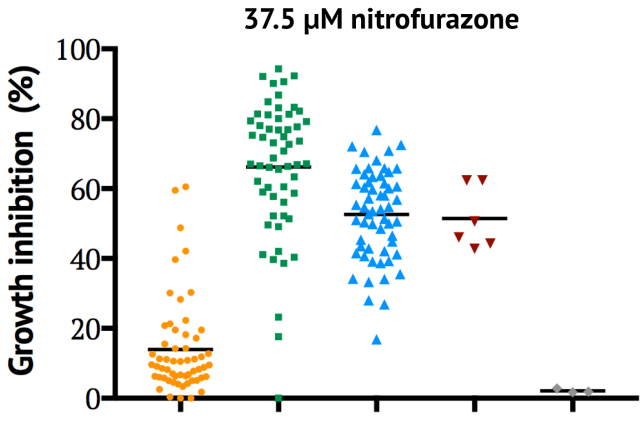

C

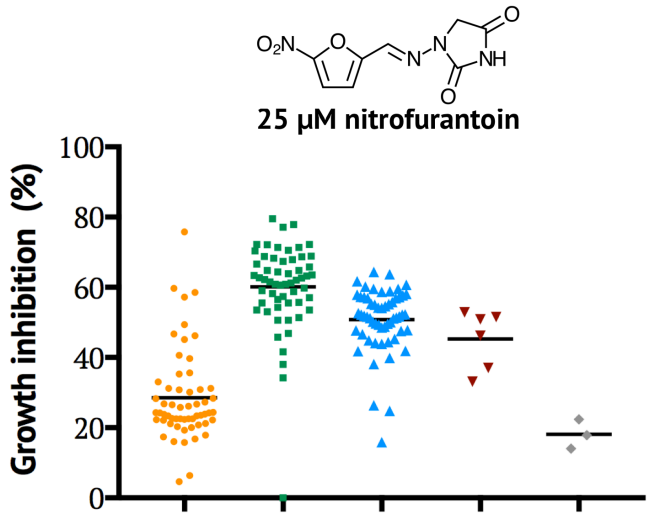

7SM variants no niclosamide

7SM variants $0.5 \mu \mathrm{M}$ niclosamide

7SM variants $5 \mu \mathrm{M}$ niclosamide

$\nabla$ NfsA_Ec in low-expressing pUCX

Empty low-expressing pUCX 
D

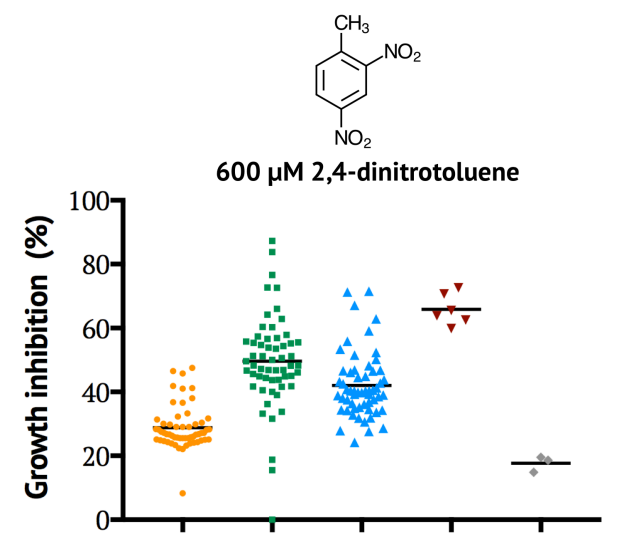

E

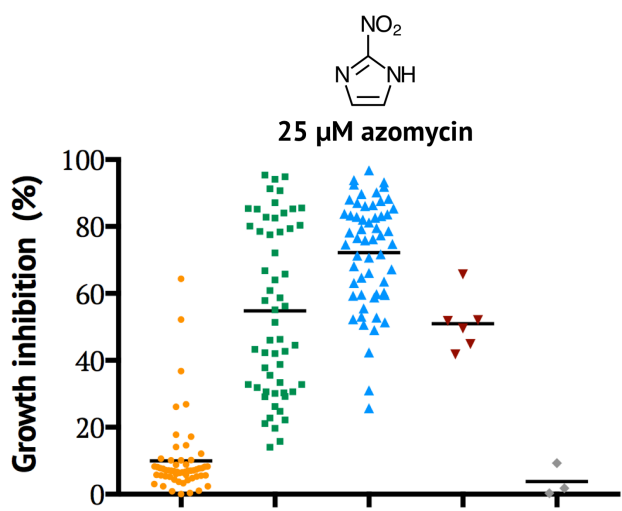

$\mathbf{F}$

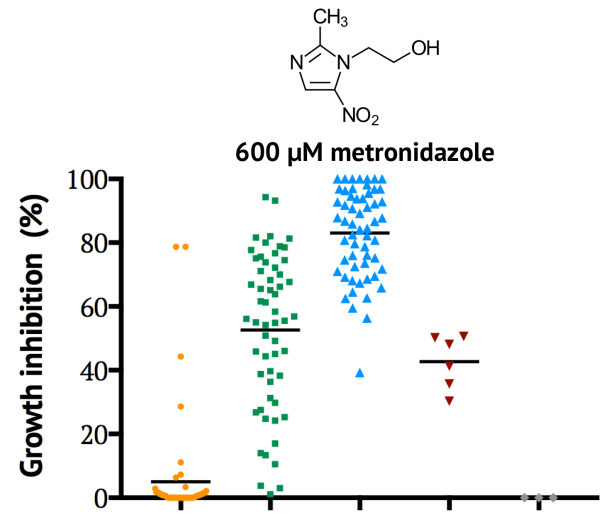

Figure 5-10 Nitroaromatic compound mediated growth inhibition of NfSA_Ec 7SM library variants in $7 N T$. Growth inhibition was monitored by measuring strain turbidity $\left(O D_{600}\right)$ pre- and post-4 hour incubation with each nitroaromatic compound. Percentage growth inhibition relative to unchallenged controls was determined. The growth inhibition of 57 variants from the NfSA_Ec 7SM library with no prior exposure to niclosamide (orange), 57 variants selected on $0.5 \mu \mathrm{M}$ niclosamide (green) and 57 variants selected on $5 \mu \mathrm{M}$ niclosamide (blue) were compared to that of NfSA_Ec in low-expressing pUCX (purple) and empty low-expressing pUCX controls (grey) following challenge with A) $500 \mu \mathrm{M}$ CB1954; B) $37.5 \mu M$ nitrofurazone; C) $25 \mu M$ nitrofurantoin; D) $600 \mu M$ 2,4-

dinitrotoluene; E) $25 \mu \mathrm{M}$ azomycin; or F) $600 \mu \mathrm{M}$ metronidazole. Data are the average of 3 biological replicates (error bars omitted for clarity). The mean growth inhibition is shown by the black horizontal line for each set of data. 
Average growth inhibition (\%)

\begin{tabular}{|c|c|c|c|c|c|}
\hline $\begin{array}{l}\text { Nitroaromatic } \\
\text { compound }\end{array}$ & $\begin{array}{l}57 \text { NfsA_Ec } \\
7 \text { SM library } \\
\text { variants not } \\
\text { selected on } \\
\text { niclosamide }\end{array}$ & $\begin{array}{c}57 \mathrm{Nfs} \text { A_Ec } \\
7 \mathrm{SM} \text { library } \\
\text { variants } \\
\text { selected on } \\
0.5 \mu \mathrm{M} \\
\text { niclosamide }\end{array}$ & $\begin{array}{c}57 \text { NfsA_Ec } \\
7 \text { SM library } \\
\text { variants } \\
\text { selected on } \\
5 \mu \mathrm{M} \\
\text { niclosamide }\end{array}$ & $\begin{array}{c}\text { NfsA_Ec in } \\
\text { low- } \\
\text { expressing } \\
\text { pUCX }\end{array}$ & $\begin{array}{l}\text { Empty } \\
\text { pUCX }\end{array}$ \\
\hline $\begin{array}{l}500 \mu \mathrm{M} \\
\mathrm{CB} 1954\end{array}$ & $13.9 \pm 6.7$ & $33.9 \pm 14.2$ & $34.7 \pm 14.3$ & $42.6 \pm 5.5$ & $\begin{array}{c}8.9 \pm \\
2.9\end{array}$ \\
\hline $\begin{array}{c}37.5 \mu \mathrm{M} \\
\text { nitrofurazone }\end{array}$ & $13.9 \pm 13.5$ & $66.2 \pm 19.2$ & $52.6 \pm 13.0$ & $51.4 \pm 8.9$ & $\begin{array}{c}2.1 \pm \\
0.6\end{array}$ \\
\hline $\begin{array}{c}25 \mu \mathrm{M} \\
\text { nitrofurantoin }\end{array}$ & $28.6 \pm 13.0$ & $60.1 \pm 12.3$ & $50.8 \pm 9.1$ & $45.3 \pm 8.3$ & $\begin{array}{c}18.1 \pm \\
4.2\end{array}$ \\
\hline $\begin{array}{c}600 \mu \mathrm{M} 2,4- \\
\text { DNT }\end{array}$ & $28.8 \pm 6.8$ & $49.6 \pm 14.7$ & $42.0 \pm 10.1$ & $65.9 \pm 4.9$ & $\begin{array}{c}17.7 \pm \\
2.4\end{array}$ \\
\hline $\begin{array}{c}25 \mu \mathrm{M} \\
\text { azomycin }\end{array}$ & $10.0 \pm 11.0$ & $54.8 \pm 25.2$ & $72.2 \pm 16.1$ & $51.0 \pm 8.3$ & $\begin{array}{c}3.8 \pm \\
4.8\end{array}$ \\
\hline $\begin{array}{c}600 \mu \mathrm{M} \\
\text { metronidazole }\end{array}$ & $5.0 \pm 15.8$ & $52.7 \pm 24.2$ & $83.1 \pm 12.9$ & $42.7 \pm 8.4$ & $\begin{array}{c}0.0 \pm \\
0.0\end{array}$ \\
\hline
\end{tabular}

Table 5-4 Average nitroaromatic compound mediated growth inhibition of NfsA_Ec 7SM library variants in 7NT. The average growth inhibition of 7NT cells overexpressing $57 \mathrm{NfS} \mathrm{A}_{-}$Ec $7 \mathrm{SM}$ library variants either with no prior exposure to niclosamide, selected on $0.5 \mu \mathrm{M}$ niclosamide or selected on $5 \mu \mathrm{M}$ niclosamide, following exposure to nitroaromatic compounds, were compared to growth inhibition of $7 N T$ cells over-expressing $N f_{S} A_{-} E c$ in low-expressing $p U C X$ or an empty $p U C X$ control (Figure 5-10). Data are the average of 3 biological replicates and errors represent \pm SEM.

In general, NfsA_Ec 7SM library variants that had been pre-selected on 0.5 $\mu \mathrm{M}$ niclosamide exhibited improved activity (as determined by their ability to sensitise $7 \mathrm{NT}$ cells to lethal concentrations of these compounds) with all six nitroaromatic compounds over non-selected 7SM variants. Furthermore, 7SM variants that could detoxify $5 \mu \mathrm{M}$ niclosamide exhibited on average higher levels of activity with azomycin and metronidazole than variants selected on $0.5 \mu \mathrm{M}$ niclosamide (Table 5-4).

In contrast, the average growth inhibition of 7NT cells bearing $5 \mu \mathrm{M}$ niclosamide-selected 7SM variants was lower than 7NT cells containing 0.5 $\mu \mathrm{M}$ niclosamide-selected variants with nitrofurazone, nitrofurantoin and 2,4-dinitrotoluene, suggesting that the stronger niclosamide selection was somewhat detrimental to enzyme activity with these substrates. 
For both CB1954 and 2,4-dinitrotoluene the average growth of 7NT cells bearing 7SM variants selected on either $0.5 \mu \mathrm{M}$ or $5 \mu \mathrm{M}$ niclosamide was still lower than that of 7NT cells bearing wild-type NfsA_Ec, suggesting that niclosamide selection of the 7SM library does not overtly aid as a selection tool for more active enzymes with these two substrates (nevertheless, it should be noted that - particularly for CB1954 - only a small number of niclosamide-selected variants needed to be examined to identify ones having greater activity than wild type NfsA_Ec, suggesting that there is still great value in use of niclosamide as a pre-selection tool). In contrast, the average growth inhibition of 7NT cells over-expressing $5 \mu \mathrm{M}$ niclosamideselected 7SM variants with azomycin or metronidazole was 1.4-fold and 1.9fold higher respectively than that of 7NT cells over-expressing wild-type NfsA_Ec, suggesting that niclosamide selection directly enriches for improved enzymes with these two substrates. Finally, 7NT cells bearing an empty pUCX plasmid exhibited over 15\% growth inhibition with the compounds nitrofurantoin and 2,4-dinitrotoluene, suggesting high inherent toxicity of these compounds even in the absence of nitro-reduction, and reflecting the relatively higher levels of growth inhibition observed in 7NT cells over-expressing 7SM variants not selected on niclosamide with these two compounds.

\subsubsection{Heatmap and sequencing of $5 \mu M$ niclosamide-selected NfSA_Ec 7SM variants}

To allow for the observation of particular amino acid preferences at each of the seven targeted residues in 7SM variants following niclosamide selection, sequencing was conducted on all $5 \mu \mathrm{M}$ niclosamide-selected 7SM variants screened in Section 5.3.2.3. Sequencing and growth inhibition results (Figure 5-10) are summarised in Table 5-5 below. The naming convention for $0.5 \mu \mathrm{M}$ and $5 \mu \mathrm{M}$ niclosamide-selected variants from this point onwards will include the niclosamide concentration on which the variant was selected, followed by its location on the 96-well plate in subsequent screens 
e.g. the $10^{\text {th }}$ variant selected on $0.5 \mu \mathrm{M}$ niclosamide was named 0.5 - 10 . The variant 5_39 was the only one for which sequencing results could not be determined, due to the co-expression of two different 7SM variants caused by the rare event of co-transformation and maintenance of both plasmids in the $7 \mathrm{NT}$ cells.

Two variants, 5_8 and 5_16 shared the same amino acid sequence (S41H/F42Y/K222R/S224N/R225H/F227V), but induced slightly different levels of growth inhibition in 7NT cells following challenge with nitrofurantoin, with 5_16 showing two-fold higher levels of growth inhibition with this compound. This could reflect some variability in the growth inhibition assays conducted and highlights the necessity of more sensitive growth inhibition $\left(\mathrm{IC}_{50}\right)$ assays when accurate determination of the ability of variants to sensitise $7 \mathrm{NT}$ cells to lethal concentrations of these compounds is required. Trends in amino acid preferences will be discussed in Section 5.3.5. 


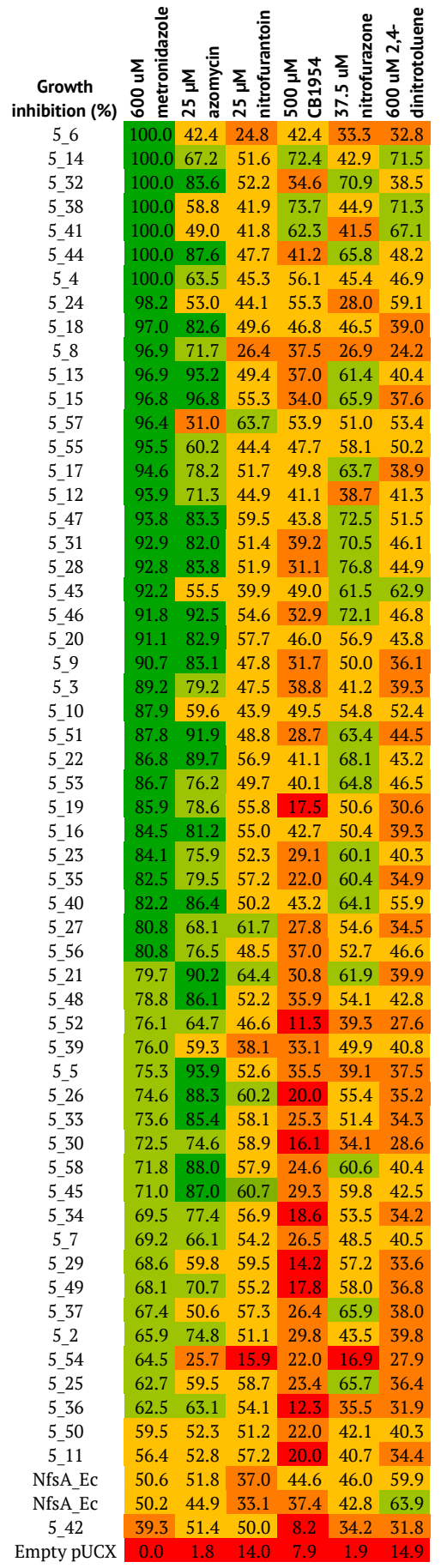

80-100\% growth inhibition
60-80\% growth inhibition
$40-60 \%$ growth inhibition
$20-40 \%$ growth inhibition
$0-20 \%$ growth inhibition

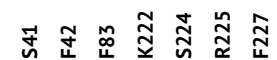

$\begin{array}{llllllll}I & F & L & V & S & V & H\end{array}$

$\begin{array}{llllllll}\mathrm{N} & \mathrm{F} & \mathrm{V} & \mathrm{T} & \mathrm{R} & \mathrm{N} & \mathrm{S}\end{array}$

$\begin{array}{lllllll}H & F & I & Q & G & N & R\end{array}$

$\begin{array}{llllllll}\text { N } & \text { Y } & \text { L } & \text { R } & \text { R } & \text { N } & \text { H }\end{array}$

$\begin{array}{lllllllll}\mathrm{N} & \mathrm{Y} & \mathrm{Y} & \mathrm{K} & \mathrm{F} & \mathrm{N} & \mathrm{N}\end{array}$

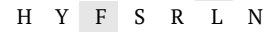

$\begin{array}{lllllll}R & Y & F & L & D & S & F\end{array}$

$\begin{array}{lllllll}\mathrm{N} & \mathrm{H} & \mathrm{F} & \mathrm{C} & \mathrm{C} & \mathrm{H} & \mathrm{N}\end{array}$

$\begin{array}{llllllll}\text { H } & \text { Y } & \text { L } & \text { R } & \text { G } & \text { N } & \text { L }\end{array}$

$\begin{array}{llllllll}\mathrm{H} & \mathrm{Y} & \mathrm{F} & \mathrm{R} & \mathrm{N} & \mathrm{H} & \mathrm{V}\end{array}$

$\begin{array}{llllllll}\text { H } & \text { F } & \text { Y } & \text { S } & \text { S } & \text { L } & \text { S }\end{array}$

$\begin{array}{llllllll}H & L & F & R & L & N & R\end{array}$

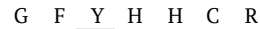

$\begin{array}{lllllll}R & Y & F & V & Y & G & N\end{array}$

\begin{tabular}{lllllllll} 
C & H & F & R & H & C & H \\
\hline
\end{tabular}

$\begin{array}{lllllllll}\text { Y } & \text { F } & R & \text { E } & \text { Y } & \text { N } & \text { N }\end{array}$

$\begin{array}{lllllll}\mathrm{R} & \mathrm{H} & \mathrm{L} & \mathrm{S} & \mathrm{S} & \mathrm{N} & \mathrm{H}\end{array}$

$\begin{array}{lllllll}H & \text { L } & \text { L } & \text { D } & \text { Y } & \text { F } & \text { D }\end{array}$

$\begin{array}{llllllll}\text { H } & \text { S } & \text { F } & \text { L } & \text { S } & \text { C } & \text { H }\end{array}$

$\begin{array}{lllllll}\mathrm{N} & \mathrm{N} & \mathrm{F} & \mathrm{N} & \mathrm{D} & \mathrm{Y} & \mathrm{H}\end{array}$

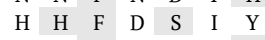

$\begin{array}{lllllll}\text { R } & \text { L } & \text { F } & \text { F } & \text { L } & \text { S } & \text { H }\end{array}$

$\begin{array}{llllllll}\text { Y } & \text { F } & \text { L } & \text { I } & \text { R } & \text { H } & \text { N }\end{array}$

$\begin{array}{lllllll}\text { H } & \text { N } & \text { L } & \text { Y } & \text { L } & \text { N } & \text { S }\end{array}$

\begin{tabular}{llllllll}
$\mathrm{N}$ & $\mathrm{N}$ & $\mathrm{F}$ & $\mathrm{K}$ & $\mathrm{F}$ & $\mathrm{H}$ & $\mathrm{H}$ \\
\hline
\end{tabular}

$\begin{array}{lllllll}\text { H } & \text { N } & \text { F } & \text { R } & \text { N } & \text { S } & \text { H }\end{array}$

$\begin{array}{lllllll}\text { F } & \text { L } & \text { H } & \text { Q } & \text { I } & \text { N } & \text { H }\end{array}$

$\begin{array}{lllllll}R & \text { L } & \text { F } & \text { P } & \text { H } & \text { G } & \text { S }\end{array}$

$\begin{array}{lllllll}\mathrm{H} & \mathrm{C} & \mathrm{F} & \mathrm{Q} & \mathrm{N} & \mathrm{F} & \mathrm{D}\end{array}$

$\begin{array}{lllllll}\mathrm{H} & \mathrm{Y} & \mathrm{F} & \mathrm{R} & \mathrm{N} & \mathrm{H} & \mathrm{V}\end{array}$

$\begin{array}{lllllll}\mathrm{Y} & \mathrm{S} & \mathrm{F} & \mathrm{L} & \mathrm{N} & \mathrm{N} & \mathrm{S} \\ \mathrm{Y} & \mathrm{F} & \mathrm{C} & \mathrm{L} & \mathrm{H} & \mathrm{R} & \mathrm{S}\end{array}$

$\begin{array}{llllllll}\text { Y } & \text { F } & \text { C } & \text { L } & \text { H } & \text { R } & \text { S }\end{array}$

$\begin{array}{lllllll}\text { R } & \text { N } & \text { L } & \text { K } & \text { D } & \text { V } & \text { D }\end{array}$

$\begin{array}{lllllllll}Y & Y & L & R & S & F & R\end{array}$

$\begin{array}{llllllll}\text { F } & F & C & S & R & R & D\end{array}$

$\begin{array}{llllllll}F & F & F & R & D & R & F\end{array}$

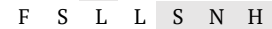

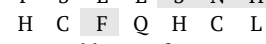

Double transformant

$\begin{array}{llllllll}\mathrm{H} & \mathrm{N} & \mathrm{L} & \mathrm{K} & \mathrm{G} & \mathrm{D} & \mathrm{R}\end{array}$

\begin{tabular}{llllllll} 
Y & H & L & S & F & N & H \\
\hline
\end{tabular}

\begin{tabular}{lllllll}
\hline & N & L & Q & H & I & G
\end{tabular}

$\begin{array}{lllllll}\mathrm{R} & \mathrm{N} & \mathrm{Y} & \mathrm{L} & \mathrm{H} & \mathrm{N} & \mathrm{H}\end{array}$

$\begin{array}{lllllll}\mathrm{H} & \mathrm{H} & \mathrm{F} & \mathrm{T} & \mathrm{N} & \mathrm{R} & \mathrm{N}\end{array}$

\begin{tabular}{lllllll} 
F & $H$ & F & T & S & G & S \\
\hline & & &
\end{tabular}

$\begin{array}{lllllll}Y & H & F & G & D & S & G\end{array}$

$\begin{array}{llllllll}\mathrm{Y} & \mathrm{H} & \mathrm{F} & \mathrm{S} & \mathrm{S} & \mathrm{S} & \mathrm{V}\end{array}$

$\begin{array}{llllllll}\text { Y } & \text { N } & \text { F } & \text { G } & \text { D } & \text { L } & \text { L }\end{array}$

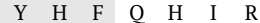

$\begin{array}{lllllll}\text { Y } & \text { H } & \text { F } & \text { F } & \text { C } & \text { L } & \text { H }\end{array}$

$\begin{array}{llllllll}\text { Y } & \text { N } & \text { L } & \text { E } & \text { F } & \text { N } & \text { D }\end{array}$

$\begin{array}{lllllll}\mathrm{Y} & \mathrm{S} & \mathrm{Y} & \mathrm{W} & \mathrm{N} & \mathrm{F} & \mathrm{D}\end{array}$

$\begin{array}{lllllll}Y & H & F & N & S & F & N\end{array}$

$\begin{array}{lllllll}Y & \mathrm{H} & \mathrm{F} & \mathrm{H} & \mathrm{H} & \mathrm{S} & \mathrm{I}\end{array}$

$\begin{array}{llllllll}Y & H & F & R & R & I & R\end{array}$

$\begin{array}{llllllll}Y & N & F & P & R & S & S\end{array}$

$\begin{array}{lllllllll}\text { Y } & \text { H } & \text { L } & \text { R } & \text { S } & \text { N } & \text { Y }\end{array}$

Most frequently occuring amino acid residue at this site

Table 5-5 Sequencing results and percentage growth inhibition of $5 \mu \mathrm{M}$ niclosamideselected NfSA_Ec 7SM variants with six different nitroaromatic compounds. Growth inhibition data is duplicated from Figure 5-10 (standard deviations omitted for clarity). Enzymes are ranked by activity with metronidazole. Amino acid occurrences at each of the seven site saturation sites in the 7SM library are listed for each variant. The most frequently occurring amino acid at each residue across all selected variants is highlighted grey. 


\subsubsection{Counter-screen of improved metronidazole-activating NfsA_Ec 7SM} library variants with S33

Growth inhibition assays conducted with 7NT cells bearing 7SM variants and the prodrug metronidazole showed a substantial number of variants exhibiting higher levels of activity than wild-type NfsA_Ec (Figure 5-10 C). As we had previously determined a moderate correlation between the activity of nitroreductases with metronidazole or S33 (Figure 5-7), the 7SM variants conferring the highest levels of growth inhibition in E. coli 7NT cells following challenge with metronidazole were subjected to determination of their activity with $\mathrm{S} 33$ by growth inhibition $\left(\mathrm{IC}_{50}\right)$ assays.

\subsubsection{Growth inhibition (IC 50 ) assays with NfSA_EC 7SM library variants with metronidazole and S33}

Fifteen NfsA_Ec 7SM variants that had been selected on $5 \mu \mathrm{M}$ niclosamide and two 7SM variants that had been selected on $0.5 \mu \mathrm{M}$ NCS showed the ability to induce over 93\% growth inhibition in 7NT following challenge with $600 \mu \mathrm{M}$ metronidazole (Table 5-5 and Figure 5-10 C). These variants were re-transformed into fresh 7NT cells, and protein expression levels were determined on an SDS-PAGE gel to confirm similar levels of expression to NfsA_Ec in low-expressing pUCX (data not shown) prior to more specific evaluation of their ability to sensitise $E$. coli to lethal concentrations of metronidazole or S33 (Table 5-6).

With the exception of 7SM variant $0.5 \_37$, which could only sensitise 7NT cells to a 1.4-fold lower concentration of metronidazole than wild-type NfsA_Ec and induced an over two-fold worse $\mathrm{IC}_{50}$ value with $\mathrm{S} 33$ than NfsA_Ec, all selected variants showed a >1.4-fold improvement in sensitising E. coli to S33 or $>9$-fold improvement in sensitisation to metronidazole. The two NfsA_Ec 7SM variants that appeared most active with S33, 5_4 and 5_6, sensitised 7NT cells to 7-fold and 4-fold lower concentrations of S33, respectively, than NfsA_Ec. The variant 5_4 had five amino acid substitutions (S41R/F42Y/K222L/S224D/R225S), with wild-type residues at 
F83 and F227, whereas 5_6 possessed a different set of five substitutions (S41I/F83L/K222V/R225V/F227H) and had wild-type residues at F42 and S224. These two variants also exhibited the highest improvements in activity with metronidazole, sensitising 7NT cells to nearly 95-fold lower (5_4) and 77-fold lower (5_6) concentrations of metronidazole than wild type NfsA_Ec. 


\begin{tabular}{|c|c|c|c|c|c|c|c|c|c|c|c|}
\hline \multirow[b]{2}{*}{ Variant } & \multirow[b]{2}{*}{$\begin{array}{c}\text { S33 IC } 50 \\
(\mu \mathrm{M})\end{array}$} & \multirow[b]{2}{*}{ 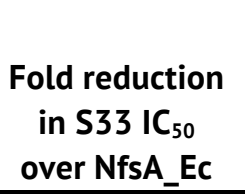 } & \multirow[b]{2}{*}{$\begin{array}{c}\text { Metronidazole } \\
\mathrm{IC}_{50}(\mu \mathrm{M})\end{array}$} & \multirow[b]{2}{*}{$\begin{array}{l}\text { Fold reduction in } \\
\text { metronidazole IC } 50 \\
\text { over NfsA_Ec }\end{array}$} & \multirow[b]{2}{*}{ S41 } & \multirow[b]{2}{*}{ F42 } & \multicolumn{5}{|c|}{ aa residue substitution } \\
\hline & & & & & & & F83 & K222 & S224 & R225 & F227 \\
\hline 54 & $14.1 \pm 2.5$ & 7.0 & $7.8 \pm 2.3$ & 94.6 & $\mathrm{R}$ & $\mathrm{Y}$ & WT & $\mathrm{L}$ & $\mathrm{D}$ & $\mathrm{S}$ & WT \\
\hline $5 \_6$ & $22.5 \pm 2.5$ & 4.4 & $9.4 \pm 1.0$ & 77.8 & I & WT & $\mathrm{L}$ & $\mathrm{V}$ & WT & $\mathrm{V}$ & $\mathrm{H}$ \\
\hline $5 \overline{55}$ & $17.6 \pm 2.8$ & 3.7 & $26.6 \pm 2.5$ & 27.6 & $\mathrm{R}$ & $\mathrm{Y}$ & WT & $\mathrm{V}$ & $\mathrm{Y}$ & G & $\mathrm{N}$ \\
\hline 538 & $28.5 \pm 0.5$ & 3.5 & $36.0 \pm 4.8$ & 20.4 & $\mathrm{~N}$ & $\mathrm{Y}$ & $\mathrm{L}$ & $\mathrm{R}$ & $\mathrm{R}$ & $\mathrm{N}$ & $\mathrm{H}$ \\
\hline $5 \_18$ & $28.2 \pm 1.7$ & 3.5 & $23.5 \pm 5.8$ & 31.3 & $\mathrm{H}$ & Y & $\mathrm{L}$ & $\mathrm{R}$ & G & $\mathrm{N}$ & $\mathrm{L}$ \\
\hline$\overline{5} 8$ & $28.9 \pm 3.9$ & 3.4 & $24.9 \pm 5.7$ & 29.6 & $\mathrm{H}$ & $\mathrm{Y}$ & WT & $\mathrm{R}$ & $\mathrm{N}$ & $\mathrm{H}$ & V \\
\hline 541 & $28.9 \pm 2.6$ & 3.4 & $26.7 \pm 1.0$ & 27.6 & $\mathrm{~N}$ & Y & $\mathrm{Y}$ & WT & $\mathrm{F}$ & $\mathrm{N}$ & $\mathrm{N}$ \\
\hline 5_32 & $31.5 \pm 5.5$ & 3.1 & $26.0 \pm 15.1$ & 28.3 & $\mathrm{H}$ & I & WT & $\mathrm{Q}$ & G & $\mathrm{N}$ & $\mathrm{R}$ \\
\hline 5_15 & $32.1 \pm 3.7$ & 3.1 & $45.5 \pm 6.6$ & 16.3 & $\mathrm{H}$ & $\mathrm{L}$ & WT & $\mathrm{R}$ & $\mathrm{L}$ & $\mathrm{N}$ & $\mathrm{R}$ \\
\hline $0 . \overline{5} \_41$ & $31.8 \pm 4.6$ & 3.1 & $61.1 \pm 7.8$ & 12.0 & $\mathrm{~V}$ & $\mathrm{Y}$ & $\mathrm{L}$ & $\mathrm{R}$ & $\mathrm{C}$ & $\mathrm{N}$ & $\mathrm{C}$ \\
\hline $5 \overline{14}$ & $32.5 \pm 4.3$ & 3.0 & $35.9 \pm 3.0$ & 20.5 & $\mathrm{~N}$ & WT & $\mathrm{V}$ & $\mathrm{T}$ & $\mathrm{R}$ & $\mathrm{N}$ & $S$ \\
\hline 557 & $35.9 \pm 8.7$ & 2.7 & $82.1 \pm 23.3$ & 9.0 & G & WT & $\mathrm{Y}$ & $\mathrm{H}$ & $\mathrm{H}$ & $\mathrm{C}$ & $\mathrm{R}$ \\
\hline $5 \_44$ & $40.3 \pm 4.3$ & 2.5 & $24.2 \pm 7.2$ & 30.4 & $\mathrm{H}$ & $\mathrm{Y}$ & WT & S & $\mathrm{R}$ & $\mathrm{L}$ & $\mathrm{N}$ \\
\hline $5 \_13$ & $41.6 \pm 4.9$ & 2.4 & $44.2 \pm 11.3$ & 16.6 & $\mathrm{H}$ & WT & $\mathrm{Y}$ & $S$ & S & $\mathrm{L}$ & S \\
\hline $5 \_17$ & $60.5 \pm 7.1$ & 1.6 & $23.1 \pm 1.6$ & 31.8 & $\mathrm{C}$ & $\mathrm{H}$ & WT & $\mathrm{R}$ & $\mathrm{H}$ & $\mathrm{C}$ & $\mathrm{H}$ \\
\hline 5_24 & $72.5 \pm 2.9$ & 1.4 & $55.1 \pm 5.4$ & 13.3 & $\mathrm{~N}$ & $\mathrm{H}$ & WT & $\mathrm{C}$ & $\mathrm{C}$ & $\mathrm{H}$ & $\mathrm{N}$ \\
\hline $\begin{array}{l}\text { Low-expressing } \\
\text { NfsA_Ec }\end{array}$ & $\begin{array}{c}98.7 \pm \\
16.6\end{array}$ & 1.0 & $735.1 \pm 28.7$ & 1.0 & WT & WT & WT & WT & WT & WT & WT \\
\hline $0.5 \_\overline{3} 7$ & $>200$ & $<0.5$ & $523.7 \pm 15.4$ & 1.4 & Y & $\mathrm{Y}$ & $\mathrm{S}$ & V & $\mathrm{R}$ & $\mathrm{N}$ & Y \\
\hline Empty pUCX & $>>200$ & - & >>2000 & - & & & & & & & \\
\hline
\end{tabular}

Table 5-6 IC 50 values for S33 or metronidazole mediated growth inhibition of E. coli 7NT cells bearing lead variants from the NfSA_EC 7SM library. Growth inhibition was monitored by measuring strain turbidity $\left(O D_{600}\right)$ pre- and post-four hour incubation across a two-fold dilution series of S33 (top concentration of $200 \mu \mathrm{M}$ ) or metronidazole (top concentration of $2 \mathrm{mM}$ ). Percentage growth relative to unchallenged controls was determined and used to calculate the concentration at which 50\% growth inhibition was observed, using GraphPad Prism 7.0. Data are the average of 3 biological replicates $\pm S D$. Enzymes are ranked by activity with $S 33$. 
A log-log correlation was conducted on the ability of these 16 7SM variants and NfsA_Ec in low-expressing pUCX to sensitise 7NT cells to S33 or metronidazole, to determine if the strong correlation observed with wildtype nitroreductases from the 58 nitroreductase library (Figure 5-7) held true here (Table 5-6).

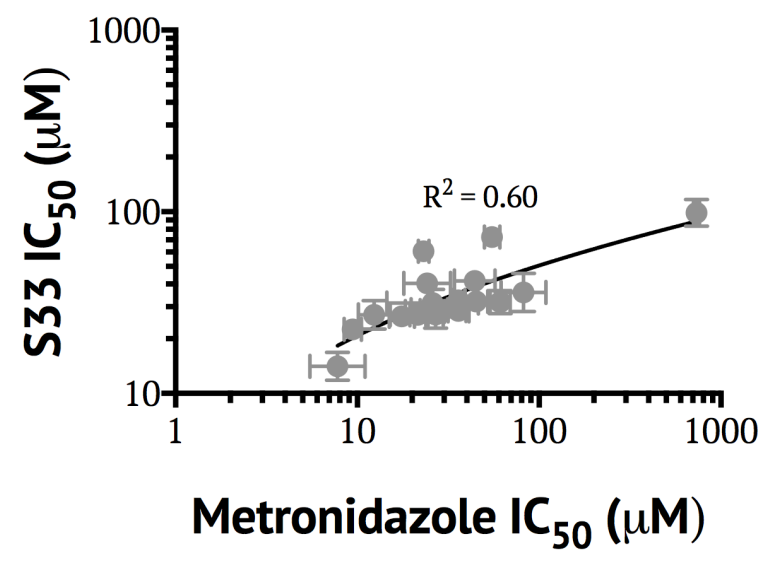

Figure 5-11 Log-log correlation between $I C_{50}$ growth inhibitory effects of S33 and metronidazole in $7 N T$ NfsA_Ec over-expression strains. IC $C_{50}$ values of $7 N T$ cells over-expressing different NfSA_Ec 7SM library variants or wild-type NfsA_Ec (all in low-expressing pUCX) following challenge with up to $200 \mu M S 33$ or $2000 \mu M$ metronidazole in $I_{50}$ assays (data from Table 5-6).

A moderate correlation $\left(\mathrm{R}^{2}=0.60\right)$ was observed with metronidazole and S33 $\mathrm{IC}_{50}$ values, confirming that the screening of metronidazole-active 7SM variants provided an enrichment for variants that were also active with S33. 


\subsubsection{Active site modelling for NfsA_Ec 7SM variants 5_4 and 5_6}
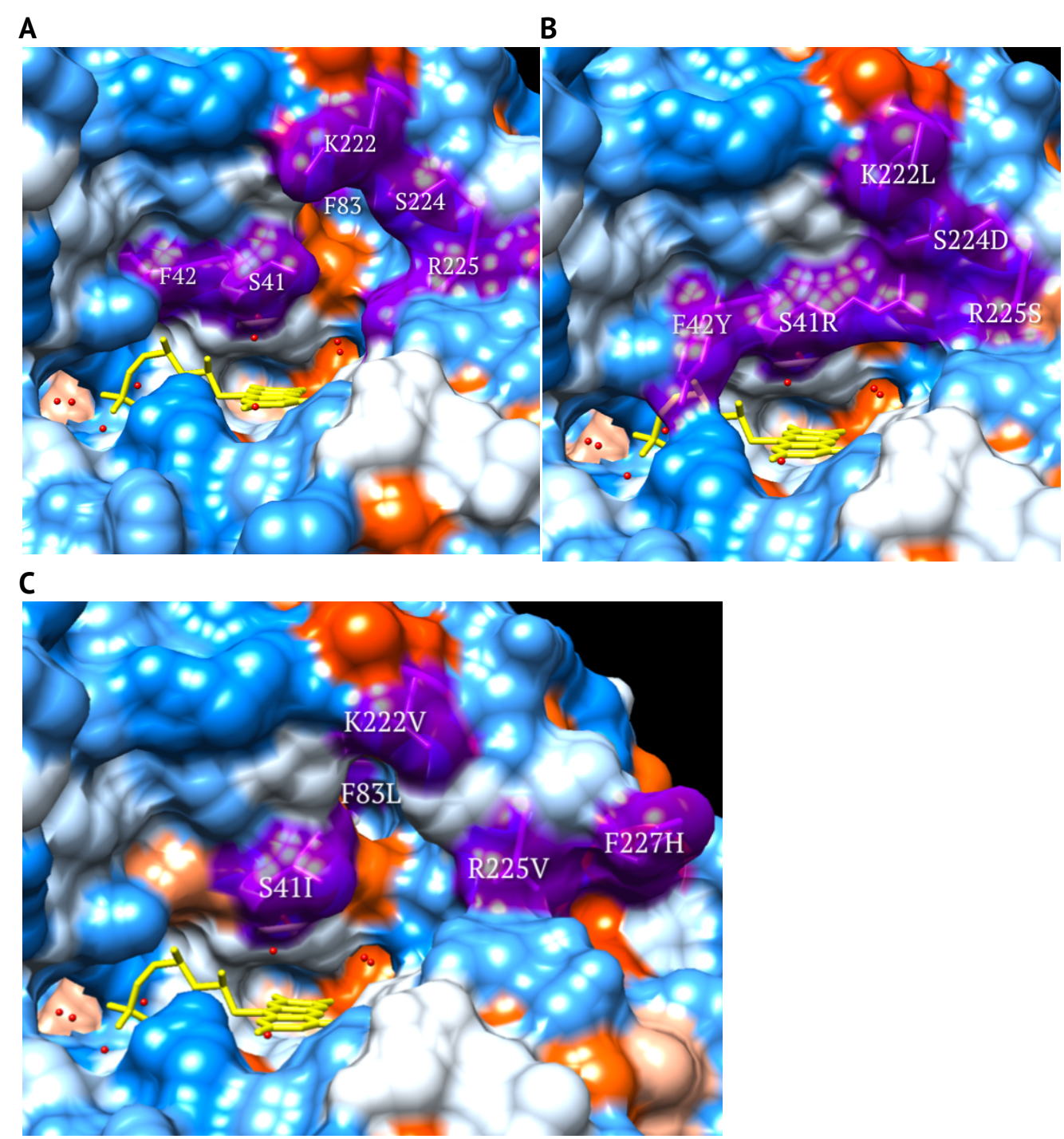

Figure 5-12 Modelling of active site of NfsA_Ec and 7SM library variants. A) Modelling of all the active site residues in wild type NfSA_Ec that were targeted for site saturation mutagenesis in the 7SM library. Target residues are highlighted in purple, and the FMN molecule is coloured yellow. B) Modelling of the active site of 7SM variant 5_4. C) Modelling of the active site of 7SM variant 5_6.

Our modelling of the active site of variant 5_4 shows the substitution of Phe at residue 42 for the slightly larger hydrophobic amino acid Tyr, whereas at residue 41 the small, uncharged Ser is replaced with a much larger, positively charged Arg (Figure 5-12 A,B). At the right-hand side of the active site, Lys at 222 and Arg at 225 are replaced with the much smaller amino acids Leu and Ser respectively, whereas uncharged Ser at 224 is 
substituted with the negatively charged Asp. The overall result is a smaller, more sterically hindered active site, which might play a role in facilitating tighter binding of metronidazole or S33 substrates.

The active site of $5 \_6$ is quite different to both 5_4 and NfsA_Ec (Figure 5-12C). Ser at position 41 is replaced with a hydrophobic amino acid, Ile, and the native Phe at position 83 is substituted with the slightly smaller Leu. At the right-hand cleft both the large, positive amino acids Lys at 222 and Arg at 225 are replaced with the much smaller, hydrophobic Val, whereas at 227 hydrophobic Phe is replaced with a positively charged His, with the overall result being a larger, much more open active site. Although highly speculative, it is possible that this architecture facilitates access of the substrates to the active site.

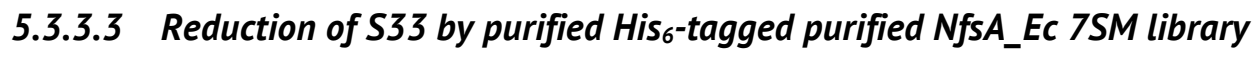 variant proteins}

To confirm the significant improvements in activity observed in the NfsA_Ec 7SM variants 5_4 and 5_6, they were re-cloned into pET28a+ in BL21 to allow His $_{6}$-tagged protein purification for the determination of in vitro kinetic parameters with S33.

Preliminary results indicated that both 5_4 and 5_6 showed substantially lower $k_{c a t}$ and $K_{M}$ values with S33 than wild-type NfsA_Ec, however they both appeared to exhibit at least a 4 -fold improvement in $k_{\text {cat }} / K_{M}$ (Table 5-7). Due to low levels of background reduction of NADPH still occurring due to incomplete removal of oxygen using the glucose oxidase-catalase oxidase scavenging system, accurate $K_{M}$ values could not be derived for either 7SM variant, and the high error bars in the measurement of initial velocities for both variants (Figure 5-13) mean that only a rough estimate of apparent $k_{\text {cat }}$ and $K_{M}$ with $\mathrm{S} 33$ could be determined. 
A

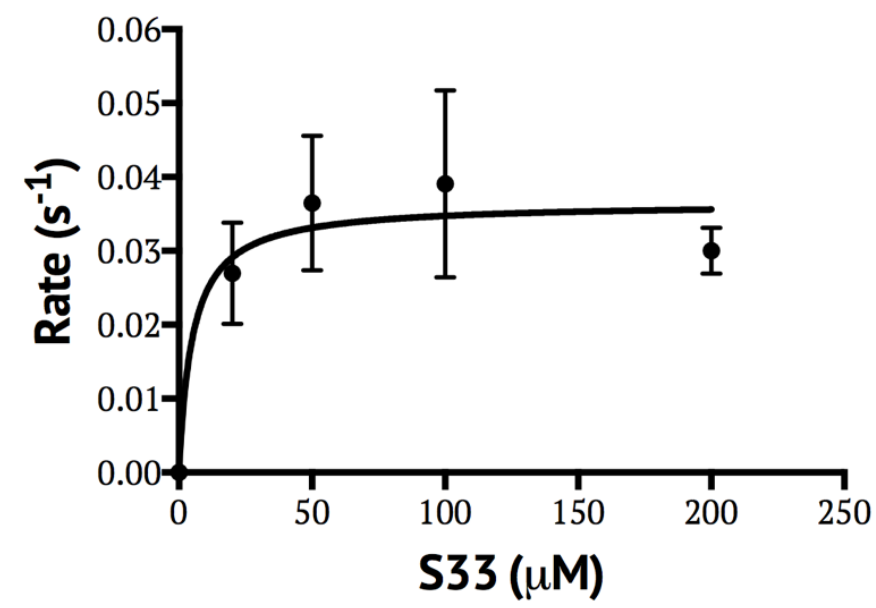

B

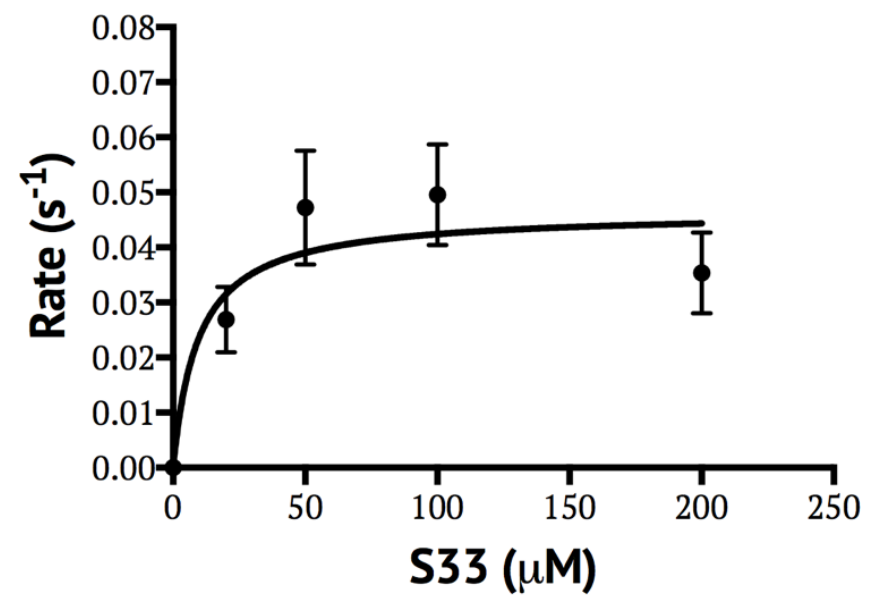

Figure 5-13 Kinetic parameters for the reduction of S33 by purified (His 6 -tagged) NfSA_Ec 7SM library variants 5_4 and 5_6. Initial rates of reaction of $A$ ) $5 \_4$ or B) 5_6, with varying concentrations of S33 (0-200 $\mu M)$ and $250 \mu M N A D P H$ with a glucose oxidase-catalase oxygen scavenging system (12 units. $\mathrm{mL}^{-1}$ glucose oxidase, 12 units. $\mathrm{mL}^{-1}$ catalase, $50 \mathrm{mM}$ glucose) were monitored at $350 \mathrm{~nm}$. At each concentration of S33 rates were measured in quadruplicate and errors represent $\pm S D$. 


\begin{tabular}{c|c|c|c|c} 
& & & \multicolumn{2}{c}{$\begin{array}{c}\text { NADPH oxidase } \\
\text { activity }(\mu \mathrm{M}\end{array}$} \\
Enzyme & $\boldsymbol{k}_{\text {cat }}\left(\mathbf{s}^{-1}\right)$ & $\boldsymbol{K}_{M}(\boldsymbol{\mu M})$ & $\begin{array}{c}\mathbf{k}_{\text {cat }} / \mathbf{K}_{\mathbf{M}} \\
\left(\mathbf{s}^{-1} \cdot \mathbf{M}^{-1}\right)\end{array}$ & $\begin{array}{c}\text { NADPH. } \boldsymbol{M M} \\
\left.\text { enzyme }^{-1} \cdot \mathbf{s}^{-1}\right)\end{array}$ \\
\hline 7SM 5_4 & $0.04 \pm 0.01$ & $\leq 15$ & $\geq 2700$ & $0.10 \pm 0.02$ \\
7SM 5_6 & $0.05 \pm 0.01$ & $\leq 20$ & $\geq 2500$ & $0.27 \pm 0.04$ \\
NfsA_Ec & $2.0 \pm 0.4$ & $3700 \pm 1100$ & $530 \pm 200$ & $0.22 \pm 0.04$
\end{tabular}

Table 5-7 Michaelis-Menten kinetic parameters estimated for NfSA_EC 7SM library variants. Initial rates of reduction were monitored at $350 \mathrm{~nm}$ at varying S33 concentrations and a fixed concentration of excess NADPH (250 $\mu M)$. Reaction rates at each $S 33$ concentration were measured in at least triplicate in the presence of a glucose oxidase-catalase oxygen scavenging system (12 units. $\mathrm{mL}^{-1}$ glucose oxidase, 12 units. $\mathrm{mL}^{-1}$ catalase, $50 \mathrm{mM}$ glucose). Apparent $K_{M}$ and $k_{\text {cat }}$ at $250 \mu M N A D P H$ were calculated using GraphPad 7.0. An extinction coefficient of $9,650 \mathrm{M}^{-1} \mathrm{~cm}^{-1}$ was used to determine rates of nitroreductase-mediated reduction of both $S 33$ and NADPH and errors represent \pm SEM. NADPH oxidase activity shows enzyme-mediated NADPH oxidation in the absence of a glucose oxidasecatalase oxygen scavenging system, measured using an extinction coefficient of $4020 \mathrm{M}^{-1} \mathrm{~cm}^{-1}$. Kinetic parameters for wild-type $N f A_{-}$Ec are duplicated from Table 3-6.

\subsubsection{Evaluation of chloramphenicol as a positive selection tool for active nitroreductases}

\subsubsection{Chloramphenicol selection of the $\mathbf{5 8}$ nitroreductase library}

As mentioned in Section 5.1.2, we also sought to determine if the use of alternative nitroreductase positive selection compounds might lead to the selection of 7SM variants exhibiting higher levels of activity with certain nitroaromatic substrates. To decide if chloramphenicol was suitable for this purpose, we first determined if nitroreductases from the 58 nitroreductase library in the 7NT strain were capable of detoxifying chloramphenicol in liquid growth assays (Figure 5-14). 


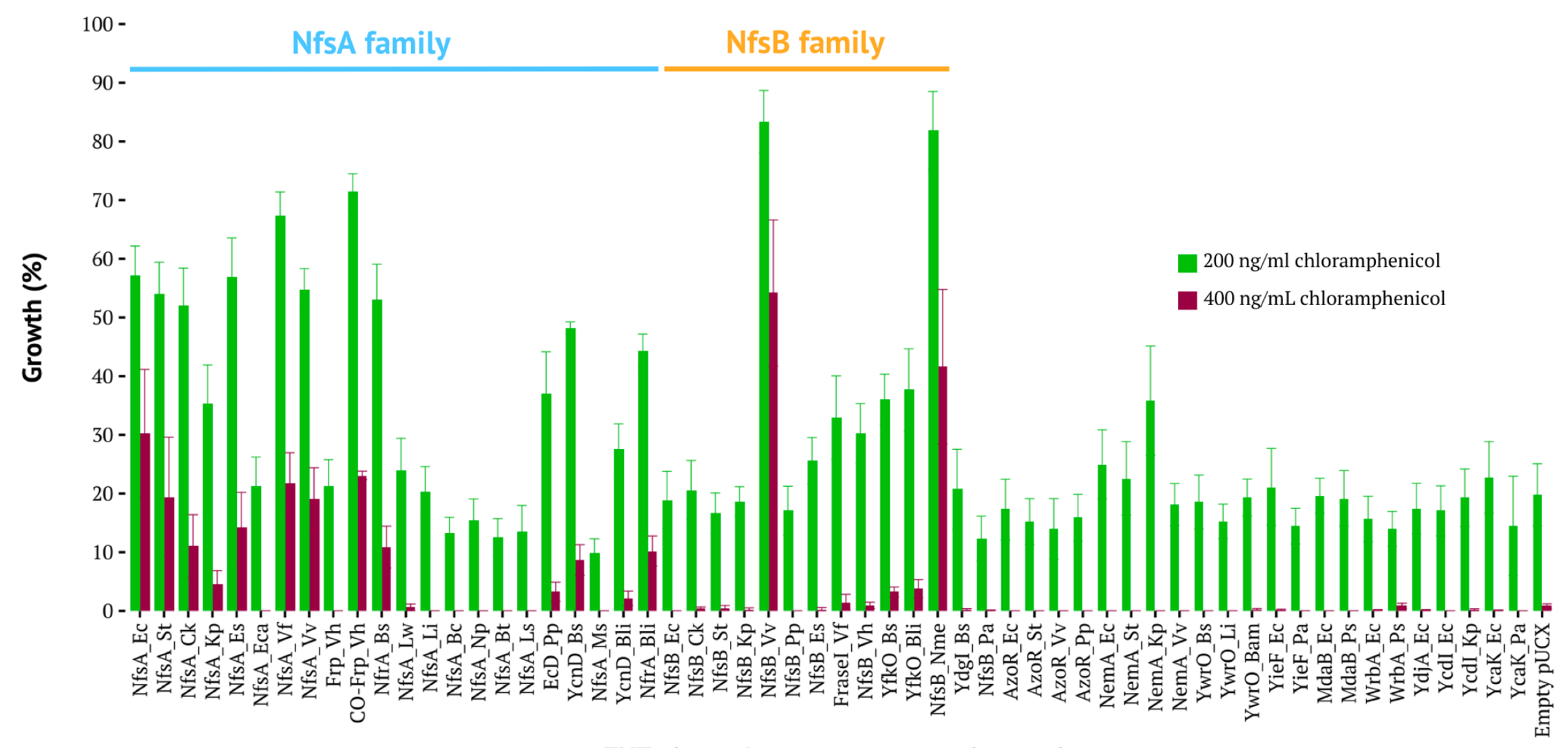

7NT nitroreductase overexpressing strain

Figure 5-14 Growth of 7NT nitroreductase over-expression strains, post-challenge with chloramphenicol. Growth of strains resistant to chloramphenicol was monitored by measuring culture turbidity $\left(\mathrm{OD}_{600}\right)$ pre-and post-four hour incubation with $200 \mathrm{ng} / \mathrm{mL}$ (green bars) or $400 \mathrm{ng} / \mathrm{mL}$ (red bars) chloramphenicol. Percentage growth relative to unchallenged controls was determined. Data are the average of 3 biological replicates \pm SEM. 
A much lower protective effect was observed for nitroreductases from the 58 nitroreductase library against chloramphenicol as compared to niclosamide, with $20 \%$ growth of the 7NT empty pUCX plasmid still observed at 200 $\mathrm{ng} / \mathrm{mL}$ chloramphenicol, whereas $400 \mathrm{ng} / \mathrm{mL}$ chloramphenicol caused complete inhibition of 7NT cells over-expressing most nitroreductases in the library. At this higher concentration of chloramphenicol, 7NT cells bearing the nitroreductase most capable of detoxifying this antibiotic, NfsB_Vv (from V. vulnificus), showed around 50\% growth, whereas those cells over-expressing NfsA_Ec exhibited 70\% growth inhibition.

\subsubsection{Chloramphenicol selection of 7NT cells over-expressing NfsA_Ec 7SM library variants}

At $1 \mu \mathrm{g} / \mathrm{mL}$ chloramphenicol, no growth of 7NT cells bearing NfsA_Ec in lowexpressing pUCX was observed on LB agar plates. When the 7SM library was plated on this concentration of chloramphenicol, $0.05 \%$ of variants were found to be capable of detoxifying the compound, yielding colonies that could be picked after 40 hours incubation at $37^{\circ} \mathrm{C}$. 57 chloramphenicolselected colonies were selected for further activity analysis with the six nitroaromatic compounds that the niclosamide-selected libraries had previously been screened against (Section 5.3.2.3).

\subsubsection{Effects of chloramphenicol selection on activity of NfSA_Ec 7SM variants with other nitroaromatic compounds}

Chloramphenicol-selected NfsA_Ec 7SM library variants were subjected to growth inhibition using the same concentrations of nitroaromatic compounds as in Section 5.3.2.3, along with two 7NT NfsA_Ec controls and an empty pUCX control, and compared to inhibition data generated for the 57 non-selected 7SM variants in Section 5.3.2.3. 
A

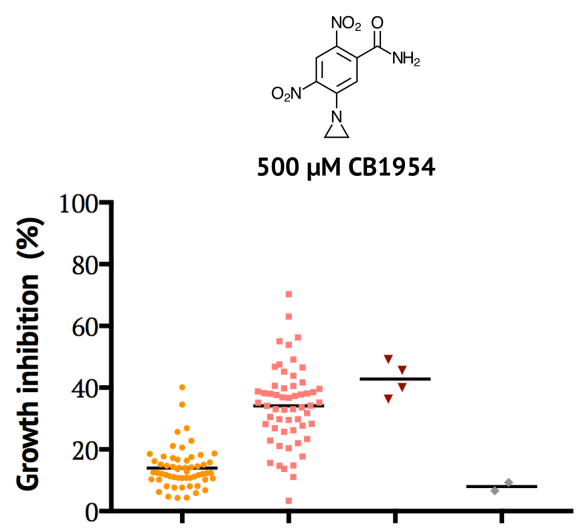

B<smiles>NC(=O)NN=Cc1ccc([N+](=O)[O-])o1</smiles>

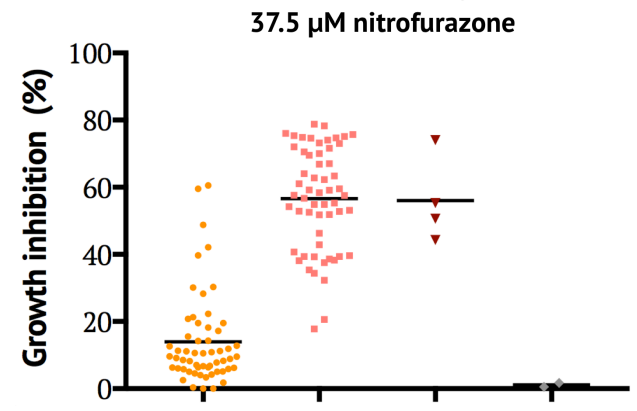

C

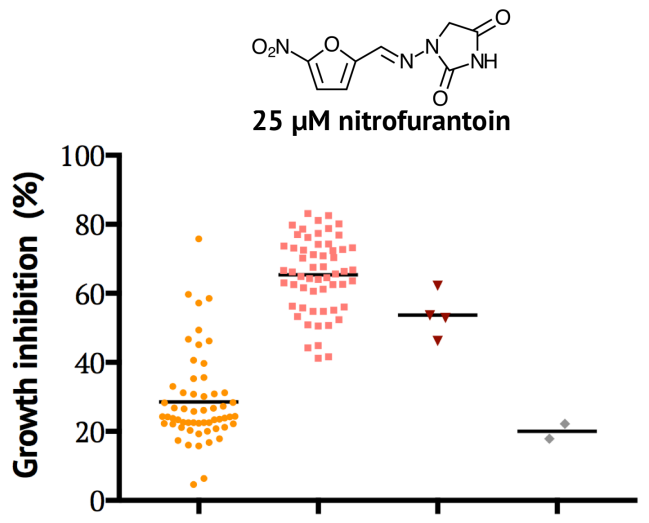

7SM variants no chloramphenicol

7SM variants $1 \mu \mathrm{g} / \mathrm{mL}$ chloramphenicol

$\checkmark$ NfsA_Ec in low-expressing pUCX

Empty low-expressing pUCX 
D

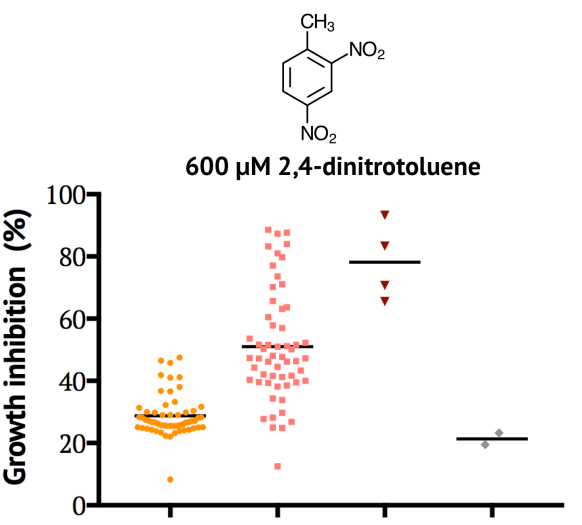

E
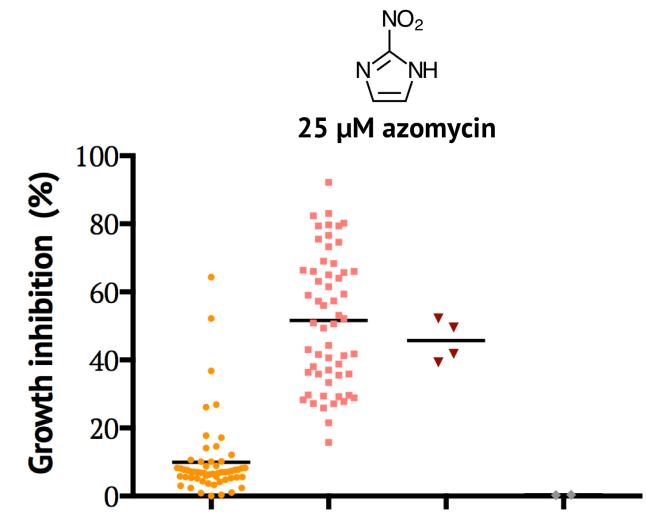

$\mathbf{F}$

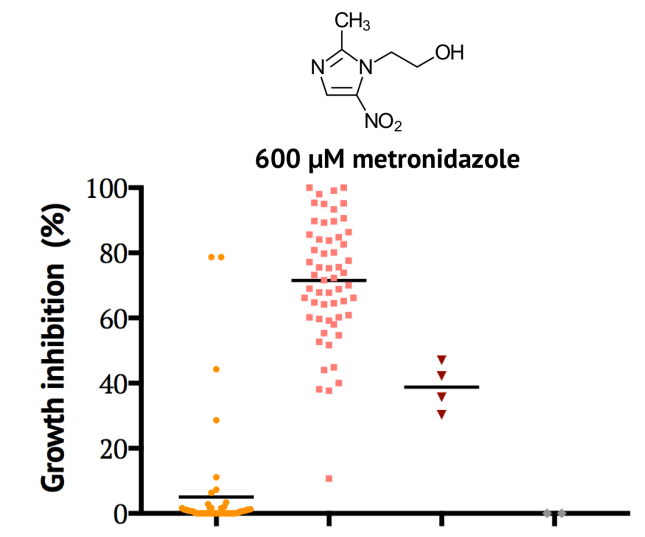

Figure 5-15 Nitroaromatic compound mediated growth inhibition of 7NT cells over-expressing NfSA_Ec 7SM library variants. Growth inhibition was monitored by measuring strain turbidity $\left(O D_{600}\right)$ pre- and post-4 hour incubation with each nitroaromatic compound. Percentage growth inhibition relative to unchallenged controls was determined. The growth inhibition of 57 variants from the NfSA_Ec $7 S M$ library having no prior exposure to chloramphenicol (orange; duplicated from Figure 5-10), and 57 variants selected on $1 \mathrm{mg} / \mathrm{mL}$ chloramphenicol (pink) were compared to that of NfSA_Ec in low-expressing pUCX (purple) and empty low-expressing pUCX controls (grey) following challenge with A) $500 \mu M$ CB1954; B) $37.5 \mu M$ nitrofurazone; C) $25 \mu M$ nitrofurantoin; D) $600 \mu M$ 2,4-dinitrotoluene; E) $25 \mu M$ azomycin; or F) $600 \mu \mathrm{M}$ metronidazole. Data are the average of 3 biological replicates (error bars omitted for clarity). The mean growth inhibition is shown by the black horizontal line for each set of data. 
Average growth inhibition (\%)

\begin{tabular}{|c|c|c|c|c|}
\hline \multirow[b]{2}{*}{$\begin{array}{l}\text { Nitroaromatic } \\
\text { compound }\end{array}$} & \multicolumn{4}{|c|}{ 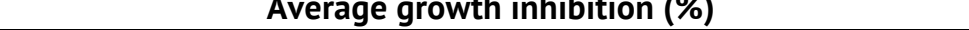 } \\
\hline & $\begin{array}{l}57 \text { NfsA_Ec } 7 \mathrm{SM} \\
\text { library variants } \\
\text { not selected on } \\
\text { chloramphenicol }\end{array}$ & $\begin{array}{l}57 \text { NfsA_Ec 7SM } \\
\text { library variants } \\
\text { selected on } \\
1 \mu \mathrm{g} / \mathrm{mL} \\
\text { chloramphenicol }\end{array}$ & $\begin{array}{l}\text { NfsA_Ec in } \\
\text { low- } \\
\text { expressing } \\
\text { pUCX }\end{array}$ & $\begin{array}{l}\text { Empty } \\
\text { pUCX }\end{array}$ \\
\hline $500 \mu \mathrm{M}$ CB1954 & $13.9 \pm 6.7$ & $34.1 \pm 12.8$ & $42.8 \pm 5.7$ & $8.0 \pm 1.9$ \\
\hline $\begin{array}{c}37.5 \mu \mathrm{M} \\
\text { nitrofurazone }\end{array}$ & $13.9 \pm 13.5$ & $56.6 \pm 15.4$ & $56.1 \pm 12.8$ & $1.1 \pm 0.8$ \\
\hline $\begin{array}{c}25 \mu \mathrm{M} \\
\text { nitrofurantoin }\end{array}$ & $28.6 \pm 13.0$ & $65.4 \pm 10.7$ & $53.7 \pm 6.6$ & $20.1 \pm 3.0$ \\
\hline $\begin{array}{c}600 \mu \mathrm{M} 2,4- \\
\text { dinitrotoluene }\end{array}$ & $28.8 \pm 6.8$ & $51.1 \pm 17.8$ & $78.2 \pm 12.6$ & $21.4 \pm 2.7$ \\
\hline $25 \mu \mathrm{M}$ azomycin & $10.0 \pm 11.0$ & $51.6 \pm 19.5$ & $45.7 \pm 6.1$ & $0.4 \pm 0.1$ \\
\hline $\begin{array}{c}600 \mu \mathrm{M} \\
\text { metronidazole }\end{array}$ & $5.0 \pm 15.8$ & $71.6 \pm 18.3$ & $38.8 \pm 7.3$ & $0.0 \pm 0.0$ \\
\hline
\end{tabular}

Table 5-8 Average nitroaromatic compound mediated growth inhibition of NfsA_Ec 7SM library variants in 7NT. The average growth inhibition of 7NT cells overexpressing $57 \mathrm{NfSA}$ Ec $7 S M$ library variants that had been selected on $1 \mu \mathrm{g} / \mathrm{mL}$ chloramphenicol, following exposure to nitroaromatic compounds, were compared to growth inhibition of 57 7SM variants with no prior exposure to chloramphenicol (duplicated from Table 5-4), 7NT cells over-expressing $N f S A_{-} E c$ in low-expressing $p U C X$ or an empty $p U C X$ control. Errors represent \pm $S D$.

Similar to the niclosamide-selected 7SM variants, the chloramphenicolselected variants exhibited improved activity over non-selected 7SM variants with all six nitroaromatic compounds. The average growth inhibition of 7NT cells bearing $1 \mu \mathrm{g} / \mathrm{mL}$ chloramphenicol-selected 7SM variants was greater than cells expressing NfsA_Ec with nitrofurantoin, metronidazole and to a lesser extent azomycin, suggesting that chloramphenicol could be used as a rapid selection tool for more active enzymes with these substrates. However with CB1954, nitrofurantoin, and 2,4-dinitrotoluene, the average growth of 7NT cells bearing chloramphenicol-selected 7SM variants was lower than that of 7NT cells bearing wild-type NfsA_Ec, suggesting that chloramphenicol selection is less overtly biased toward activity with these compounds. 


\subsubsection{Heatmap and sequencing of $1 \mu \mathrm{g} / \mathrm{mL}$ chloramphenicol-selected NfsA_Ec 7SM variants}

To allow for any trends in amino acid preference at each of the seven targeted residues in 7SM variants following chloramphenicol selection to be observed, sequencing was conducted on all $571 \mu \mathrm{g} / \mathrm{mL}$ chloramphenicolselected 7SM variants; sequencing and growth inhibition results (from Figure 5-15) are compiled in Table 5-9 below. The naming convention for chloramphenicol-selected variants from this point onwards includes Chl (short for chloramphenicol), followed by the concentration of chloramphenicol variants were selected on $(1000 \mathrm{ng} / \mathrm{mL})$ and its location on the 96 -well plate in subsequent screens e.g. the $10^{\text {th }}$ variant selected on 1 $\mu \mathrm{g} / \mathrm{mL}$ chloramphenicol was named Chl_1000_10.

No $1 \mu \mathrm{g} / \mathrm{mL}$ chloramphenicol-selected 7SM variants shared the same combination of amino acid substitutions as $5 \mu \mathrm{M}$ niclosamide-selected 7SM variants in Table 5-5. Three pairs of chloramphenicol-selected 7SM variants shared the same sequences: Chl_1000_2 and Chl_1000_3 (S41G/F42Y/K222L/S224I/F227D), Chl_1000_7 and Chl_1000_8 (S41Y/F41L/F83I/K222R/S224R/R225N/F227N), and Chl_1000_6 and Chl_1000_39 (F83N/K222R/S224D/R225H/F227S). Oddly, different levels of activity were observed between the latter two pairs of enzymes, in particular Chl_1000_8 exhibited far higher levels of activity with metronidazole than Chl_1000_7, and Chl_1000_3 showed higher levels of activity with metronidazole than Chl_1000_2. Again, this could reflect some variability in the growth inhibition assays conducted. 


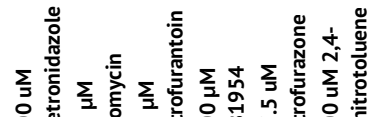

Growth

Chl_1000_6 $100.0 \quad 29.2 \quad 41.6 \quad 63.0 \quad 35.4 \quad 87.7$

$\begin{array}{ccccccc}\text { Chl_100__6 } & 100.0 & 29.2 & 41.6 & 63.0 & 35.4 & 87.7 \\ \text { Chl_1000_39 } & 100.0 & 41.6 & 60.6 & 70.3 & 59.5 & 88.6\end{array}$

\begin{tabular}{lrrrrrrr} 
Chl_100__22 & 99.1 & 79.7 & 78.6 & 46.5 & 75.1 & 60.5 \\
\hline
\end{tabular}

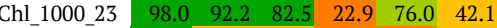

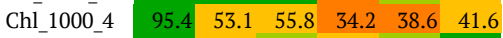

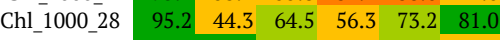

Chl_1000_20 $\quad 95.0 \quad 80.2 \quad 73.2 \quad 41.7 \quad 62.3 \quad 51.6$

$\begin{array}{llllllll}\text { Chl_1000_57 } & 93.4 & 43.0 & 50.5 & 49.1 & 63.4 & 70.2\end{array}$

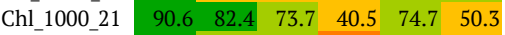

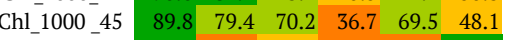

$\begin{array}{llllllll}\text { Chl_1000_8 } & 89.7 & 28.3 & 44.2 & 38.1 & 38.1 & 83.3\end{array}$

$\begin{array}{lllllll}\text { Chl_1000 29 } & 89.3 & 35.5 & 67.7 & 55.0 & 75.4 & 71.1\end{array}$

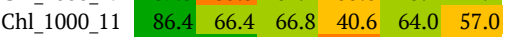

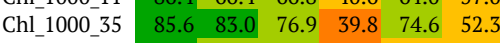

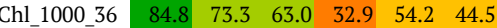

Chl_1000_16 $\quad 84.1 \quad 57.4 \quad 44.9 \quad 35.2 \quad 37.5 \quad 47.4$

$\begin{array}{lllllll}\text { Chl_1000_5 } & 83.8 & 37.0 & 67.5 & 31.8 & 54.9 & 50.2\end{array}$

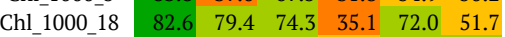

$\begin{array}{llllllll}\text { Chl_1000_43 } & 80.9 & 64.0 & 74.2 & 45.2 & 74.9 & 65.7\end{array}$

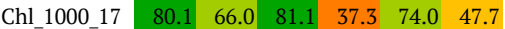

$\begin{array}{lllllllll}\text { Ch__1000_51 } & 79.8 & 66.0 & 80.1 & 33.0 & 61.0 & 41.7\end{array}$

Chl_1000_46 $77.6 \quad 29.6 \quad 65.6 \quad 47.5 \quad 75.7 \quad 84.0$

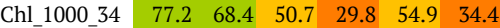

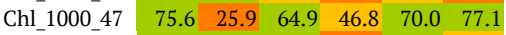

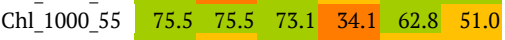

$\begin{array}{llllllll}\text { Ch__1000_14 } & 75.3 & 56.0 & 71.2 & 38.8 & 57.5 & 53.6\end{array}$

$\begin{array}{llllllll}\text { Chl 1000 24 } & 73.9 & 15.8 & 41.2 & 39.6 & 20.6 & 79.8\end{array}$

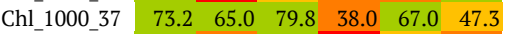

$\begin{array}{lllllllll}\text { Chl_1000_44 } & 72.3 & 28.9 & 66.6 & 14.8 & 53.1 & 26.9\end{array}$

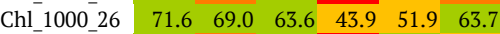

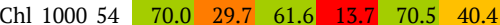

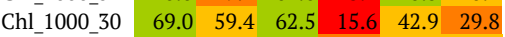

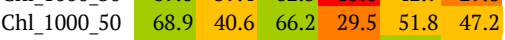

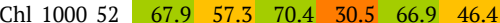

$\begin{array}{llllllll}\text { Chl } 1000 \quad 49 & 67.8 & 76.6 & 78.8 & 38.5 & 78.8 & 51.2\end{array}$

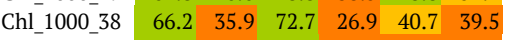

$\begin{array}{lllllll}\text { Chl_1000_3 } & 66.2 & 35.9 & 62.5 & 29.8 & 32.3 & 33.9\end{array}$

$\begin{array}{lllllll}\text { Chl } 1000 \_53 & 65.2 & 41.3 & 76.2 & 33.0 & 78.3 & 39.6\end{array}$

Chl_1000_12 $64.8 \quad 27.8 \quad 54.8 \quad 38.2 \quad 59.2 \quad 63.2$

Chl_1000_41 $64.5 \quad 21.5 \quad 55.1 \quad 33.6 \quad 58.4 \quad 51.6$

$\begin{array}{lllllll}\text { Chl_1000_9 } & 64.2 & 50.9 & 77.4 & 23.4 & 55.3 & 41.3\end{array}$

Chl_1000_13 $60.9 \quad 74.6 \quad 66.4 \quad 36.9 \quad 56.7 \quad 46.2$

Chl_1000_25 $\quad 60.2 \quad 49.4 \quad 61.2 \quad 21.1 \quad 39.6 \quad 27.8$

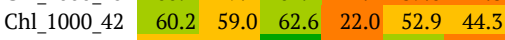

$\begin{array}{llllllll}\text { Chl_1000_33 } & 59.7 & 50.6 & 83.1 & 28.2 & 73.0 & 38.2\end{array}$

Chl_1000_56 $559.2 \quad 27.1 \quad 52.4 \quad 37.8 \quad 39.4 \quad 73.6$

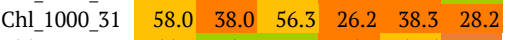

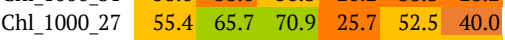

$\begin{array}{llllllll}\text { Chl_1000_7 } & 54.7 & 61.5 & 64.0 & 28.3 & 46.3 & 46.2\end{array}$

$\begin{array}{llllllll}\text { Chl_1000_19 } & 52.7 & 41.8 & 56.0 & 17.7 & 34.4 & 24.9\end{array}$

$\begin{array}{llllllll}\text { Chl_1000_15 } & 51.7 & 63.1 & 72.4 & 53.9 & 71.6 & 87.4\end{array}$

$\begin{array}{lllllll}\text { NfsA_Ec } & 47.0 & 39.3 & 62.2 & 49.1 & 74.0 & 93.4\end{array}$

$\begin{array}{llllllll}\text { Chl_1000_32 } & 44.9 & 36.4 & 77.0 & 27.7 & 59.1 & 43.4\end{array}$

$\begin{array}{lllllll}\text { Chl } 1000 \_2 & 44.0 & 29.4 & 54.8 & 37.6 & 57.6 & 57.9\end{array}$

$\begin{array}{llllllll}\text { NfsA_Ec } & 42.2 & 52.2 & 53.6 & 45.6 & 55.3 & 83.4\end{array}$

$\begin{array}{lllllll}\text { Chl_1000_10 } & 40.0 & 33.4 & 64.3 & 41.6 & 55.3 & 83.4 \\ \text { Che } & 39.4 & 25.0\end{array}$

$\begin{array}{lllllll}\text { Chl } 1000-48 & 38.1 & 52.2 & 53.3 & 20.4 & 39.3 & 38.5\end{array}$

\begin{tabular}{l|rrrrrrr} 
Chl_1000_40 & 37.7 & 38.8 & 72.5 & 14.7 & 52.8 & 39.4 \\
Chl_1000_58 & 10.7 & 27.2 & 50.9 & 3.4 & 17.8 & 12.5 \\
\hline
\end{tabular}

$\begin{array}{rrrrrrr}\text { Ch__1000_58 } & 10.7 & 27.2 & 50.9 & 3.4 & 17.8 & 12.5 \\ \text { Empty pUCX } & 0.0 & 0.4 & 22.2 & 9.3 & 0.5 & 23.3\end{array}$

80-100\% growth inhibition 60-80\% growth inhibition 40-60\% growth inhibition

$20-40 \%$ growth inhibition

$0-20 \%$ growth inhibition

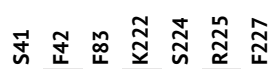

$\begin{array}{llllllll}S & F & N & R & D & H & S\end{array}$

$\begin{array}{lllllll}S & F & N & R & D & H & S\end{array}$

$\begin{array}{llllllll}R & F & V & R & G & N & D\end{array}$

$\begin{array}{lllllllllll}H & H & F & E & F & N & N\end{array}$

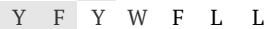

N

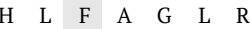

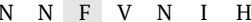

\begin{tabular}{lllllll}
$H$ & F & S & P & D & R & S \\
\hline & & &
\end{tabular}

$\begin{array}{llllllll}H & H & \text { F } & \text { T } & G & C & N\end{array}$

\begin{tabular}{llllllll} 
Y & L & I & R & R & N & $N$ \\
\hline
\end{tabular}

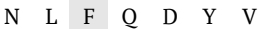

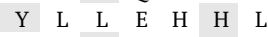

$\begin{array}{llllllll}R & L & F & V & R & H & S\end{array}$

$\begin{array}{lllllll}H & F & I & V & R & S & R\end{array}$

$\begin{array}{llllllll}H & F & S & T & Y & H & S\end{array}$

$\begin{array}{llllllll}\text { L } & \text { Y } & \text { L } & \text { Y } & \text { H } & \text { S } & \text { G }\end{array}$

$\begin{array}{llllllll}\text { H } & \text { F } & \text { L } & \text { Y } & \text { C } & \text { I } & \text { R }\end{array}$

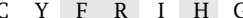

$\begin{array}{lllllll}Y & L & L & Y & R & \text { V } & D\end{array}$

$\begin{array}{lllllll}\mathrm{Y} & \mathrm{Y} & \mathrm{N} & \mathrm{S} & \mathrm{D} & \mathrm{I} & \mathrm{H} \\ \mathrm{S} & \mathrm{N} & \mathrm{F} & \mathrm{C} & \mathrm{V} & \mathrm{H} & \mathrm{H}\end{array}$

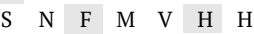

$\begin{array}{lllllll}F & L & F & Q & G & D & L\end{array}$

$\begin{array}{lllllll}\mathrm{N} & \mathrm{S} & \mathrm{F} & \mathrm{R} & \mathrm{F} & \mathrm{N} & \mathrm{C}\end{array}$

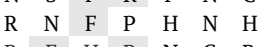

$\begin{array}{llllllll}R & F & H & R & N & C & R\end{array}$

$\begin{array}{lllllll}S & F & F & N & \text { I } & \text { L } & \text { D }\end{array}$

$\begin{array}{llllllll}\text { Y } & \text { Y } & \text { L } & G & F & \text { D } & \text { N }\end{array}$

$\begin{array}{llllllll}N & C & \text { F } & \text { L } & \text { C } & \text { D } & \text { N }\end{array}$

$\begin{array}{lllllll}R & N & N & E & S & S & D\end{array}$

$\begin{array}{lllllllll}N & \mathrm{C} & \mathrm{F} & \mathrm{K} & \mathrm{H} & \mathrm{H} & \mathrm{Y}\end{array}$

$\begin{array}{lllllll}\text { H } & \text { C } & \text { F } & \text { L } & \text { R } & \text { H } & \text { R }\end{array}$

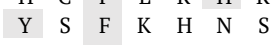

$\begin{array}{lllllll}H & S & F & D & H & \text { I } & S\end{array}$

$\begin{array}{lllllll} & H & F & T & H & F & H\end{array}$

$\begin{array}{llllllll}\text { Y } & G & F & T & R & N & S\end{array}$

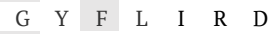

$\begin{array}{lllllll}\mathrm{Y} & \mathrm{L} & \mathrm{D} & \mathrm{O} & \mathrm{N} & \mathrm{F} & \mathrm{N}\end{array}$

$\begin{array}{llllllll}D & F & F & W & H & R & Y\end{array}$

G

\begin{tabular}{lllllll} 
Y & N & Y & Q & Y & N & D \\
\hline & L & L & S & S & S & I
\end{tabular}

\begin{tabular}{lllllll}
$H$ & L & L & S & S & S & I \\
\hline & C & F & F & S & F & H
\end{tabular}

$\begin{array}{lllllll}H & C & F & F & S & F & H\end{array}$

$\begin{array}{llllllll}\text { Y } & H & \text { V } & \text { V } & \text { N } & \text { C } & \text { D }\end{array}$

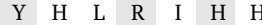

$\begin{array}{lllllll}\mathrm{N} & \mathrm{H} & \mathrm{H} & \mathrm{R} & \mathrm{N} & \mathrm{F} & \mathrm{D}\end{array}$

$\begin{array}{llllllll}\text { Y } & F & \text { I } & \text { V } & \text { L } & \text { G } & \text { Y }\end{array}$

$\begin{array}{lllllllll}\text { F } & \text { H } & \text { F } & \text { R } & \text { I } & G & D\end{array}$

$\begin{array}{lllllll}Y & L & I & R & R & N & N\end{array}$

$\begin{array}{lllllll}H & C & Y & S & G & G & N\end{array}$

$\begin{array}{llllllll}S & F & F & K & S & R & F\end{array}$

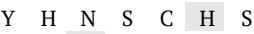

$\begin{array}{lllllll}G & \text { Y } & \text { F } & \text { L } & \text { I } & \text { R } & \text { D }\end{array}$

$\begin{array}{llllllll}\mathrm{Y} & \mathrm{F} & \mathrm{D} & \mathrm{I} & \mathrm{R} & \mathrm{Y} & \mathrm{N}\end{array}$

$\begin{array}{lllllll}H & \text { L } & \text { N } & \text { R } & \text { G } & \text { G } & \text { G }\end{array}$

$\begin{array}{llllllll}Y & N & L & A & Y & H & H\end{array}$

$\begin{array}{llllllll}H & \text { H } & \text { Y } & \text { R } & \text { D } & \text { C } & \text { S }\end{array}$

Most frequently occuring

amino acid residue at this site

Table 5-9 Sequencing results and percentage growth inhibition of $1 \mu \mathrm{g} / \mathrm{mL}$ chloramphenicolselected NfsA_Ec 7SM variants with six different nitroaromatic compounds. Growth inhibition data is duplicated from Figure 5-15 (standard deviations omitted for clarity). Enzymes are ranked in order of activity with metronidazole. Amino acid occurrences at each of the seven site saturation sites in the 7SM library are listed for each variant. The most frequently occurring amino acid at each residue across all selected variants is highlighted grey. 


\subsubsection{Effects of niclosamide or chloramphenicol selection on amino acid distribution in 7SM library variants}

Sequencing results for $5 \mu \mathrm{M}$ niclosamide- and $1 \mu \mathrm{g} / \mathrm{mL}$ chloramphenicolselected NfsA_Ec 7SM variants from Table 5-5 and Table 5-9 were compiled to determine any trends in amino acid residue substitutions for variants that could metabolise either of the positive selection compounds (see Table 5-10).

\subsubsection{Effects of $5 \mu \mathrm{M}$ niclosamide or $1 \mu \mathrm{g} / \mathrm{mL}$ chloramphenicol selection on amino acid distribution in 7SM library variants}

A

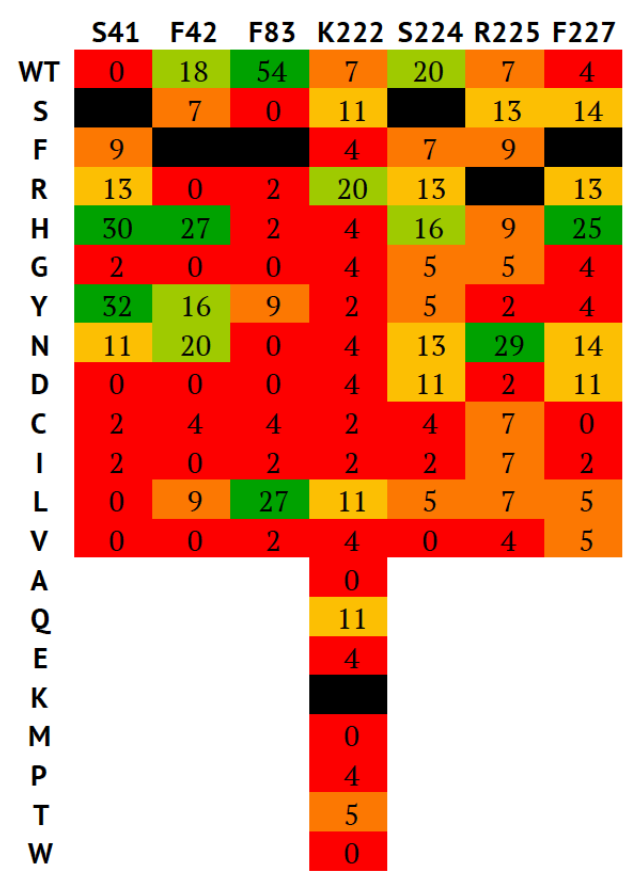

$\geqq 25 \%$ distribution at this residue $15-24 \%$ distribution at this residue $10-24 \%$ distribution at this residue $5-9 \%$ distribution at this residue $\leqq 4 \%$ distribution at this residue
B

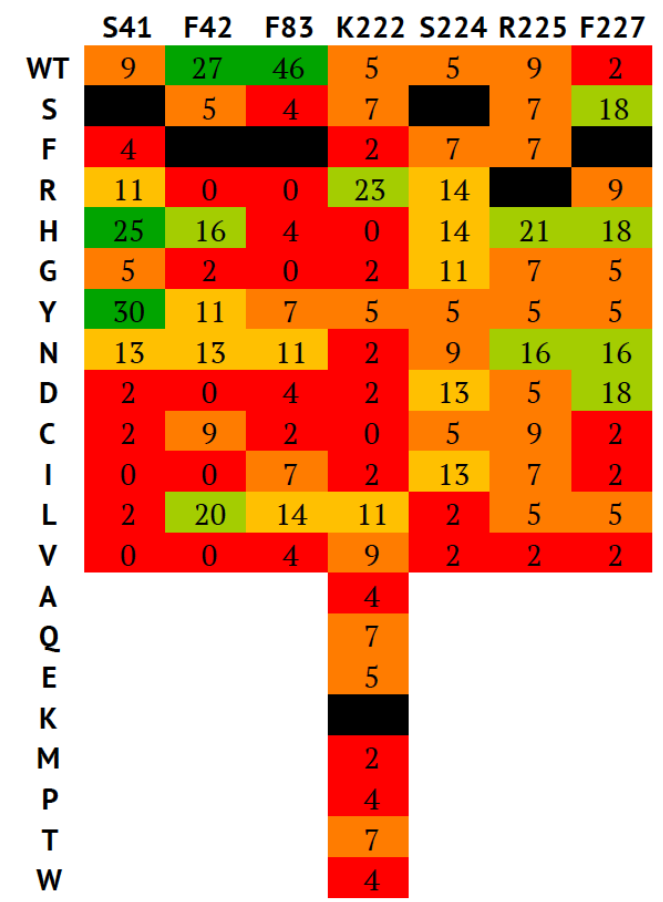

$\geqq 25 \%$ distribution at this residue $15-24 \%$ distribution at this residue $10-24 \%$ distribution at this residue 5-9\% distribution at this residue $\leqq 4 \%$ distribution at this residue

Table 5-10 Distribution of amino acids across niclosamide or chloramphenicol-selected NfSA_Ec 7SM variants. The proportion of all 57 NfSA_Ec 7SM variants selected with either A) $5 \mu \mathrm{M}$ niclosamide, or B) $1 \mu \mathrm{g} / \mathrm{mL}$ chloramphenicol containing a particular amino acid at each site-targeted residue was calculated as a percentage and colour-coded accordingly (see legend). The distribution of wild-type amino acid at each residue is shown in the top row. 
At residue 41, both niclosamide and chloramphenicol selection favoured the substitution of the larger amino acids His or Tyr for wild-type Ser. At residue 42, niclosamide selection slightly favoured the substitution of positively charged His, whereas chloramphenicol selection favoured the wild-type Phe residue. At residue 83 both niclosamide and chloramphenicol selection favoured the wild-type Phe residue. At residue 222 both niclosamide and chloramphenicol slightly favoured the substitution of Arg, a slightly larger (but still basic) amino acid, in place of wild-type Lys. At residue 224 niclosamide selection favoured wild-type Ser or positively charged His, whereas chloramphenicol selection showed no particular bias toward any residue at this positon. At residue 225, niclosamide selection strongly favoured substitution of the smaller amino acid Asn, whereas chloramphenicol selection favoured substitutions to either His or Asn (again, both smaller than the wild type Arg). Finally at residue 227 both niclosamide and chloramphenicol favoured substitutions of polar amino acids in place of hydrophobic Phe, with niclosamide selection favouring His and chloramphenicol selection favouring substitutions to Ser, His, Asn or Asp in equal measure.

\subsubsection{Changes in amino acid distribution of leading $5 \mu \mathrm{M}$ niclosamide variants}

Following the observation of general trends in amino acid substitutions of niclosamide or chloramphenicol-selected 7SM variants, we next sought to determine if any trends in amino acid distributions could be seen in the variants most active with the nitroaromatic compounds counter-screened with in Sections 5.3.2.4 and 5.3.4.4. When the $5 \mu \mathrm{M}$ niclosamide-selected 7SM variants were counter-screened with alternative nitroaromatic compounds, $>70 \%$ of variants showing increased activity (as measured by growth inhibition) over wild-type NfsA_Ec was observed when screened with metronidazole, azomycin, nitrofurantoin and nitrofurazone (Table 5-5). To determine if improved activity with a particular substrate could be correlated with a preference for an amino acid at any of the seven site 
residues, changes in percentage amino acid distribution (from Table 5-10A) in the most active 20 variants with each of these four compounds was determined (Table 5-11).

The niclosamide-selected variants most active with metronidazole showed an increased preference for His or Asn, both polar amino acids, at residue 41, with a strong disinclination for the hydrophobic Tyr. Interesting Tyr had been the most frequently occurring amino acid substitution following niclosamide selection, followed by His. At residue 42 there was selection against His and Asn, the two most frequently occurring amino acid substitutions following niclosamide selection alone, and preference for Tyr or wild-type Phe (also hydrophobic). At residue 83 there was a slight decrease in the proportion of variants having the wild-type Phe, and at residues 224, 225 and 227 there was a slight increase in the proportion of variants containing Tyr, Asn and Asn at these sites respectively.

The variants with the most activity with azomycin showed a strong preference for His at residue 41, but strongly disfavoured both Tyr and Asn. At residue 83 some preference for Leu was shown, whereas wild-type Phe (the most frequently occurring amino acid following niclosamide selection) was slightly disfavoured. At residue 222 some preference for Ser was shown, and at residue 224 there was a slight preference for smaller amino acids (Ser, Gly), whereas His was slightly disfavoured. At residue 225 Phe was disfavoured, and at residue 227 some preference for His was observed.

The leading nitrofurantoin-activating variants showed the opposite trend for amino acid preferences at residue 41 to variants active with metronidazole, with preference for hydrophobic Tyr but polar residues Asn and His were disfavoured. At residue 42, positively charged His was preferred whereas Tyr was disfavoured. At residue 222 the polar, uncharged Gln was disfavoured, and residues 224 and 225 showed a preference for and against His respectively. 


\section{A. Metronidazole}

$\begin{array}{llllllllll}\text { S41 } & \text { F42 } & \text { F83 } & \text { K222 } & \text { S224 R225 F227 }\end{array}$

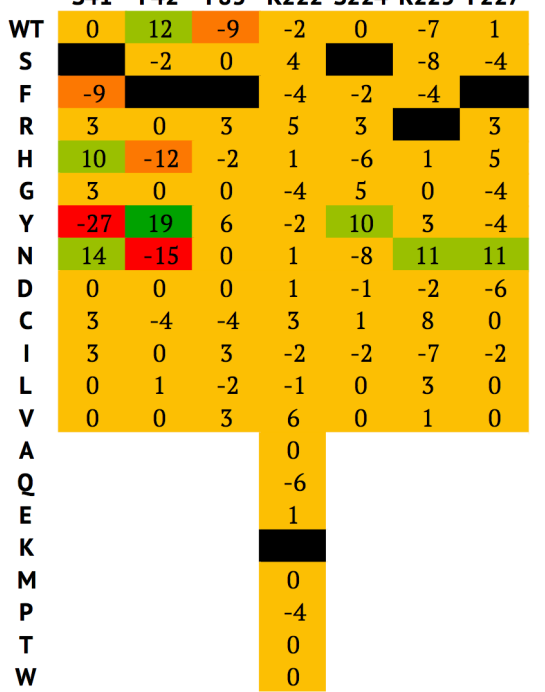

\section{Nitrofurantoin}

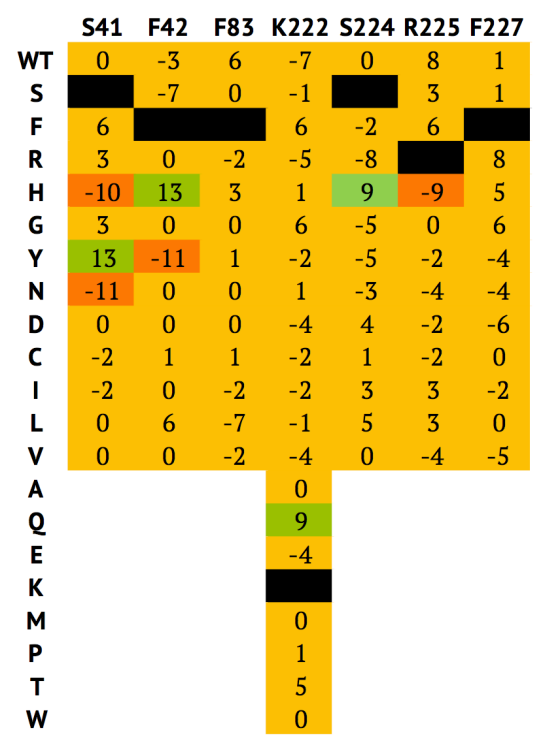

\section{B. Azomycin}

S41 F42 F83 K222 S224 R225 F227

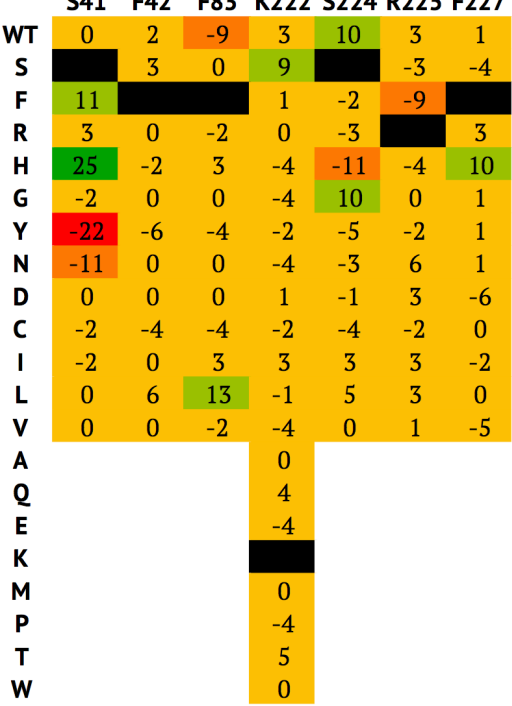

D. Nitrofurazone

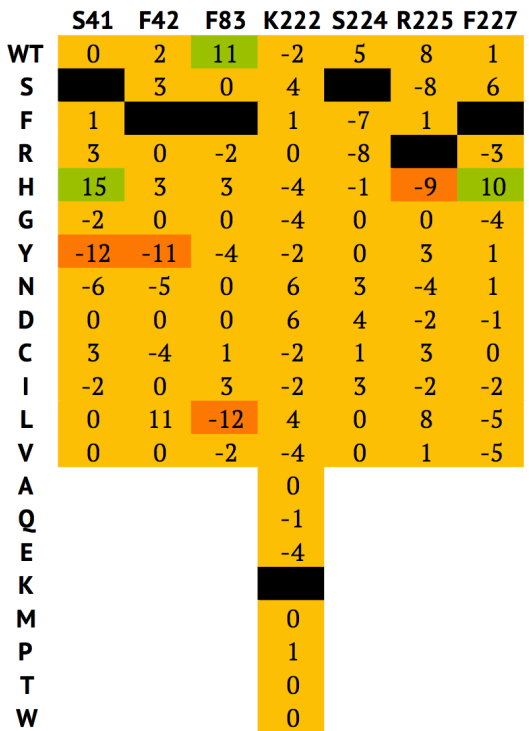

$\geqq 15 \%$ change in distribution at this residue 9-14\% change in distribution at this residue -8 to $8 \%$ change in distribution at this residue -9 to $-14 \%$ change in distribution at this residue $\leqq-15 \%$ change in distribution at this residue

Table 5-11 Changes in amino acid distribution of $5 \mu \mathrm{M}$ niclosamide-selected 7SM variants exhibiting improved activity with other nitroaromatic compounds. Amino acid distributions of the 20 most active $5 \mu M$ niclosamide-selected 7SM variants with either A) metronidazole, B) azomycin, C) nitrofurantoin, or D) nitrofurazone, was compared to that of all $5 \mu M$ niclosamide-selected variants (Table 5-10A). Changes in amino acid distribution at each site-targeted residue was calculated as a percentage and colourcoded accordingly (see legend). The distribution of wild-type amino acid at each residue is shown in the top row. 
The leading nitrofurazone-active variants showed preferences for His at residue 41 and against Tyr, and at residue 42 Tyr was also disfavoured. At residue 83 there was increased preference for wild-type Phe whereas the much smaller Leu was selected against. At residue 225 some preference for His was observed, whereas at residue 225 His was disfavoured.

\subsubsection{Changes in amino acid distribution of the leading chloramphenicol- selected variants}

We conducted the same calculations as in Section 5.3.5.3 on the 20 most active $1 \mu \mathrm{g} / \mathrm{mL}$ chloramphenicol-selected variants with metronidazole, azomycin, nitrofurantoin and nitrofurazone (Table 5-9) in attempt to determine if improved activity with these substrates could be correlated with a preference for an amino acid at any of the seven site residues (Table 5-12). The leading metronidazole-activating variants selected on chloramphenicol showed a slight preference for positively charged His at residue 41 but disfavoured hydrophobic Tyr. At residue 42, there was a strong preference for wild-type Phe, followed by the also hydrophobic Leu, and Cys was disfavoured slightly. At residue 83 wild-type Phe was disfavoured, and at residues 222 and 225, the residues Tyr and Asn respectively (neither of which were strongly represented following chloramphenicol selection) were preferred. Finally at residue 227, a slight preference for His was observed. The variants most active with azomycin exhibited slight preferences towards positively charged amino acids Arg and His at residue 41, with selection against Tyr and Asn. At residue 42 some selection for hydrophobic Leu was observed, at the cost of Cys, and there was also increased selection for Leu at residue 83 . At residue 222 there was increased selection for negatively charged Glu, and selection against Leu. At residue 224 increased selection for the very small amino acid Gly at the cost of Asn was shown. 


\section{A. Metronidazole}

$\begin{array}{lllllll}\text { S41 F42 F83 } & \text { K222 } & \text { S224 R225 F227 }\end{array}$

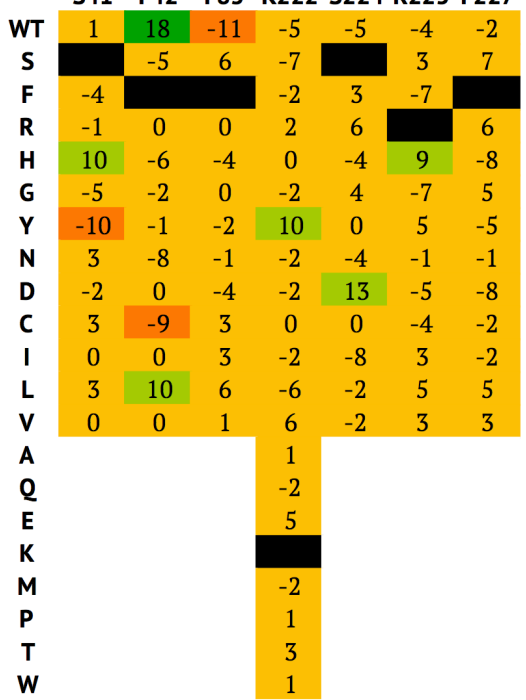

\section{Nitrofurantoin}

$\begin{array}{llllllll}\text { S41 F42 F83 } & \text { K222 } & \text { S224 R225 F227 }\end{array}$

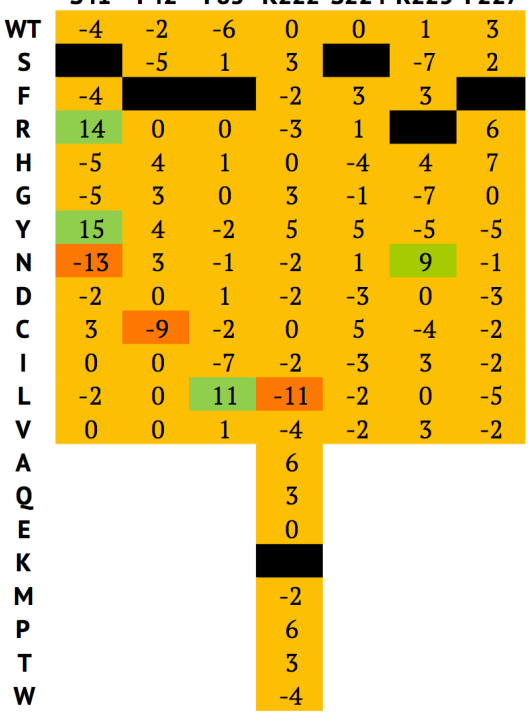

\section{B. Azomycin}

$\begin{array}{lllllllll}\text { S41 F42 F83 K222 S224 R225 F227 } & \text { F }\end{array}$

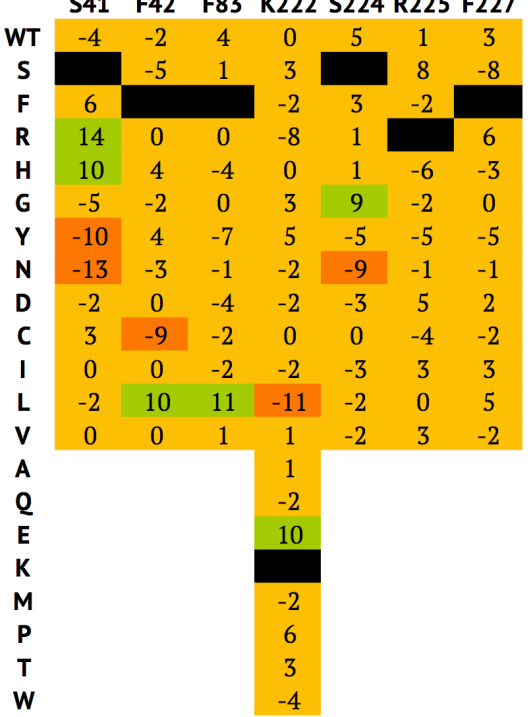

\section{Nitrofurazone}

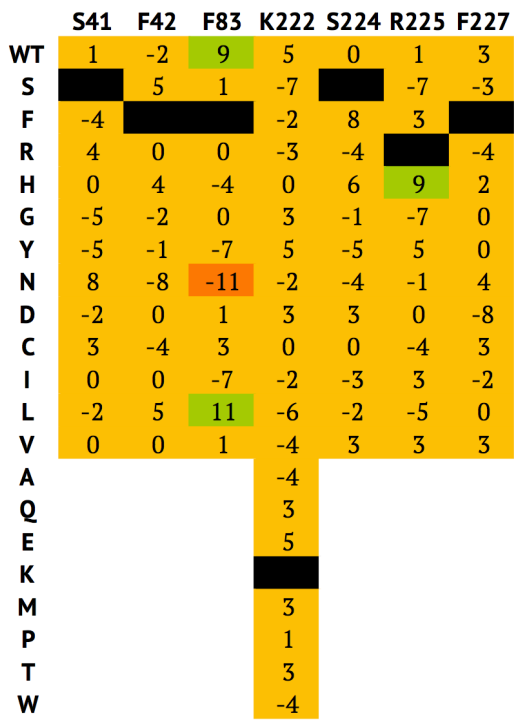

$\geqq 15 \%$ change in distribution at this residue 9-14\% change in distribution at this residue -8 to $8 \%$ change in distribution at this residue -9 to $-14 \%$ change in distribution at this residue $\leqq-15 \%$ change in distribution at this residue

Table 5-12 Changes in amino acid distribution of $1 \mu \mathrm{g} / \mathrm{mL}$ chloramphenicol-selected 7SM variants exhibiting improved activity with other nitroaromatic compounds. Amino acid distributions of the 20 most active $1 \mu \mathrm{g} / \mathrm{mL}$ chloramphenicol-selected $7 S M$ variants with either A) metronidazole, B) azomycin, C) nitrofurantoin, or D) nitrofurazone, was compared to that of all $1 \mu \mathrm{g} / \mathrm{mL}$ chloramphenicol-selected variants (see Table 5-10B). Changes in amino acid distribution at each site-targeted residue was calculated as a percentage and colour-coded accordingly (see legend). The distribution of wild-type amino acid at each residue is shown in the top row. 
The variants most active with nitrofurantoin showed a preference for Arg or Tyr at residue 41 at the cost of Asn. At residue 42 some selection against Cys was observed. Some preference for Leu was seen at residue 83, whereas at residue 222 it was selected against. At residue 225 some preference was seen for Asn.

The most active nitrofurazone-reducing variants showed for hydrophobic residues wild-type Phe and Leu and against polar Asn at residue 83, and at residue 225 some preference towards His was observed.

\subsection{Discussion}

In the research conducted for this chapter we evaluated the ability of the nitroaromatic compound niclosamide to select for variants from a large targeted site saturation NfsA_Ec library exhibiting improved activity with the novel 5-nitroimidazole PET imaging probe S33. This 7SM library randomised amino acids at seven key active site residues, and thus we expected the vast majority of variants to be non-functional; however it also gave us the potential to generate variants with extremely different active sites to that of wild-type NfsA_Ec. First, selection of $E$. coli 7NT cells overexpressing variants from the 7SM library was conducted using $0.5 \mu \mathrm{M}$ niclosamide, which was the minimum concentration that inhibited growth of 7NT cells bearing an empty pUCX plasmid. We observed selection of $4 \%$ of the 7SM library, and counter-screening of a subset of these selected variants revealed that they showed improved abilities over non-niclosamide-selected variants to sensitise $E$. coli to lethal concentrations of six structurally diverse nitroaromatic compounds. These compounds included antibiotic drugs, environmental pollutants, GDEPT prodrugs, the compound azomycin (used as a surrogate for 2-nitroimidazole imaging probes), and the 5nitroimidazole prodrug metronidazole, which was utilised as a surrogate for S33 in these preliminary assays. When the 7SM library was instead selected on $5 \mu \mathrm{M}$ niclosamide (a concentration above which 7NT cells overexpressing NfsA_Ec in low-expressing pUCX could grow), a lower proportion 
of the library $(0.5 \%)$ was selected for. These variants exhibited even greater abilities to sensitise $E$. coli to metronidazole or azomycin, suggesting a positive correlation between the ability of 7SM library variants to metabolise these compounds and niclosamide. To confirm that the variants most active with metronidazole also demonstrated superior S33-activating capabilities, more sensitive $\mathrm{IC}_{50}$ assays were conducted with these lead variants and metronidazole or S33. These assays revealed variants that could sensitise 7NT cells to up to 94-fold and 7-fold lower concentrations of metronidazole and S33 than wild-type NfsA_Ec respectively. The two lead variants, 5_4 and 5_6, each having five different amino acid substitutions, also exhibited improved abilities to activate S33 as purified proteins in vitro, with at least a 4.7-fold improvement in $k_{c a t} / K_{M}$ over wild-type NfsA_Ec. However due to incomplete scavenging of oxygen by the glucose oxidase catalase system employed for in vitro kinetics, the background NADPH oxidase activity of these enzymes meant that accurate $K_{M}$ values could not be calculated for either variant. We therefore cannot yet determine with any certainty whether these variants are more active than the lead NfsA_Ec R225A variant discovered in Chapter 3, which exhibited a 7-fold improvement in $k_{c a t} / K_{M}$ over wild-type NfsA_Ec.

Nevertheless, the improved activity of these variants with metronidazole in vivo has made them of interest for other researchers in the Ackerley lab, who are working on the generation of improved metronidazole-activating enzymes for use in targeted zebrafish ablation. They are currently being taken forward for assessment of activity with metronidazole following stable transfection into human cancer cell lines, and a new protocol utilizing an NADPH regeneration system to more accurately determine their kinetic parameters with metronidazole is currently being optimised.

We also showed that the nitroaromatic antibiotic chloramphenicol, another potential positive selection compound, was able to be detoxified both by wild-type nitroreductases from the 58 nitroreductase family and by a subset of the 7SM library. $1 \mu \mathrm{g} / \mathrm{mL}$ chloramphenicol, a concentration at which $7 \mathrm{NT}$ 
cells over-expressing NfsA_Ec in low-expressing pUCX as well as 7NT cells bearing an empty pUCX plasmid could not grow, selected for around $0.05 \%$ of the 7SM library. Counter-screening with the same panel of various nitroaromatic compounds revealed that these variants also exhibited improved capabilities to sensitise 7NT cells to these substrates over nonselected 7SM variants, and they represent another source of potentially improved S33-activating enzymes. Unfortunately due to limited supplies of S33, the variants exhibiting the highest levels of activity with metronidazole were unable to be counter-screened with S33.

The results from counter-screening of niclosamide or chloramphenicolselected 7SM variants with a panel of nitroaromatic substrates indicated that selection with a particular positive selection compound could pre-dispose a pooled group of variants for subsequent activity with different substrates. In general, a lower concentration of niclosamide $(0.5 \mu \mathrm{M})$ was more likely to select for variants exhibiting improved activity over wild-type NfsA_Ec with the nitrofuran drugs nitrofurazone or nitrofurantoin, demonstrating that it could be utilised as a sole selection agent for these compounds. A higher concentration of niclosamide $(5 \mu \mathrm{M})$ selected for variants that were in many cases much more active with the nitroimidazole compounds azomycin (a 2nitroimidazole) and metronidazole (a 5-nitroimidazole) than wild-type NfsA_Ec. Selection using $5 \mu \mathrm{M}$ niclosamide or $1 \mu \mathrm{g} / \mathrm{mL}$ chloramphenicol both enhanced the pool of 7SM variants for those exhibiting more activity with CB1954 and 2,4-dinitrotoluene; however most of these variants still exhibited less activity than wild-type NfsA_Ec. Counter-screening of more variants with these two compounds is likely necessary for the discovery of nitroreductases that are more active than the wild-type parent enzyme. The sequencing of 7SM variants that could detoxify either $5 \mu \mathrm{M}$ niclosamide or $1 \mu \mathrm{g} / \mathrm{mL}$ chloramphenicol gave us insight into the effects of selection on amino acid distributions at all seven site-targeted residues. At residue 83 around half of variants still contained wild-type Phe, suggesting that amino acid substitutions at this position did not substantially improve NfsA_Ec 
activity with either niclosamide or chloramphenicol. Weaker preferences for the wild-type amino acid was shown at all other six residues, and amino acid substitutions were preferred at residues 41, 222, 225 and 227 following niclosamide or chloramphenicol selection.

Of the 11 million unique 7SM variants transformed into $E$. coli $7 \mathrm{NT}$ cells, 5 $\mu \mathrm{M}$ niclosamide selected for around 550,000 different variants, whereas 1 $\mu \mathrm{g} / \mathrm{mL}$ chloramphenicol selected for around 55,000 unique variants. A mere small subset of 57 variants chosen from each selection pressure were sequenced, and no variant was found to be duplicated in both pools. We have yet to determine whether amino acid substitutions found in niclosamide-selected variants also allows for improvement of enzyme activity with chloramphenicol, and vice-versa.

In that vein, ongoing work conducted by other researchers in the Ackerley lab is investigating the activity of active-site randomized NfsA_Ec variants that can detoxify high concentrations of both niclosamide and chloramphenicol. To achieve this, the work described in this chapter was followed by the subsequent creation and screening of another site saturation library (the 8SM library) targeting eight other active site residues of NfsA_Ec (L43, C45, E99, L103, R133, N134, H215, T219) with niclosamide and chloramphenicol and the same panel of nitroaromatic compounds. The results from both libraries are giving the Ackerley lab insight into which residues are most amenable to amino acid substitutions, and together have informed the selection of a final panel of ten active site residues to be targeted in a novel NfsA_Ec site-saturation mutagenesis library. This library is being created and analysed as part of a Marsden grant received by $\mathrm{Dr}$ David Ackerley in 2016, seeking to use NfsA_Ec as a model for natural enzyme evolution, including the effects of applying simultaneous positive and negative selection pressures during evolution.

The results from this chapter give insight into the effectiveness of chloramphenicol and niclosamide selection of the NfsA_Ec 7SM library for the rapid discovery of variants exhibiting improved activity with a wide 
range of nitroaromatic substrates, and lends credence for the potential of these compounds as easy-to-utilise selection tools for active nitroreductase enzymes from other variant libraries. 


\section{Chapter 6. Discovery of novel nitroreductases from multi-genomic DNA}

\subsection{Introduction}

To expand our current repertoire of nitroreductase enzymes, our lab has been interested in the discovery of novel nitroreductases from alternative sources of bacterial DNA. Initially, the nitroreductases discovered and evaluated in the 1980s (from E. coli, Enterobacter cloacae and Salmonella typhimurium; Bryant and DeLuca, 1991; Bryant et al., 1981; Tatsumi et al., 1982) by other research groups all occurred in intestinal bacterial species, and the Ackerley lab has also found highly active nitroreductases in marine species (V. vulnificus, V. harveyi, A. fisheri; Prosser et al., 2013) and soildwelling bacteria (P. putida, B. thuringiensis, B. subtilis; Prosser et al., 2013). We believed soil bacteria to be a yet untapped potentially highly diverse source of novel nitroreductases, as a single gram of soil has been estimated to contain 2,000 to 18,000 distinct prokaryote genomes (Daniel, 2005). However less than $1 \%$ of soil bacteria are able to be cultured under standard laboratory conditions, and the most frequently used method to assess the entire diversity of the soil metagenome is through the cloning of suitable sizes of soil DNA into an appropriate vector and transformation into a host organism, usually E. coli (Daniel, 2005). Full-length genes encoded within these fragments of DNA can then be discovered following sequence-based or function-based screening techniques. We reasoned that creation of metagenomic libraries might enable the discovery of novel nitroreductases that exhibited higher activity with S33 than wild-type or evolved nitroreductases uncovered so far in the research described in this thesis. 


\subsubsection{Creation and screening of metagenomic libraries}

\subsubsection{Construction of metagenomic DNA libraries}

For the construction of metagenomic libraries, the DNA is first extracted either directly from the environmental sample or from recovered microbial cells (Kakirde et al., 2010). This DNA is then sheared either mechanically or by restriction endonucleases, and appropriately sized fragments of DNA are cloned into an appropriate expression vector (Daniel, 2005). Vectors utilised for cloning of DNA fragments include plasmids for smaller fragments (typically $<15 \mathrm{~kb}$ ), cosmids or fosmids for medium-length fragments (up to $45 \mathrm{~kb}$ ), or bacterial artificial chromosomes (BACs) for larger fragments of DNA (up to $200 \mathrm{~kb}$; Uchiyama and Miyazaki, 2009). Metagenomic libraries are most frequently screened in E. coli strains; however other bacteria genera including Agrobacterium, Burkholderia and Pseudomonas have also been utilised as screening hosts (Craig et al., 2010). Due to the small size of most nitroreductase genes (usually $<800 \mathrm{bp}$ ), it was reasoned that the cloning of smaller fragments of metagenomic DNA into plasmids should allow for the capturing of full-length functional nitroreductases. Furthermore, plasmids exhibit higher copy numbers than other cloning vectors, and could thus allow for the detection of weakly-expressing genes as well as the expression of metagenomic genes from vector-based promoters (discussed further in Section 6.1.2.1; Daniel, 2005).

\subsubsection{Methods for screening of metagenomic libraries}

Sequence-based screening of metagenomic libraries is most often conducted either through comparison of gene sequences (derived from next-generation sequencing) to genes of interest, or by PCR-based analysis of genes through the use of degenerate oligonucleotide primers (based on consensus amino acid sequences in enzyme families; Culligan et al., 2014). These forms of screening do not require the enzymes to be expressed by the host organism, however they only allow for the discovery of close homologues of genes that have already been annotated, and discovered genes may not exhibit the 
desired activity or the entire genes may not be fully captured within the metagenomic fragment (Daniel, 2005).

Functional screening of metagenomic libraries can also be conducted using direct selection, where the host strain requires a target protein to be expressed to enable growth on selective media (e.g. transporter proteins; Daniel, 2005), or through the screening for enzymatic activation of a substrate to a colourmetric or fluorometric product (Kakirde et al., 2010). Functional screening allows for the potential identification of completely novel genes or gene classes for which significant homologues have not yet been annotated (Daniel, 2005); however it also requires successful transcription, translation and protein folding in the host organism (Gabor et al., 2004).

\subsubsection{Expressing metagenomic genes using vector promoters}

To improve the ability of host organisms to transcribe and translate metagenomic genes, different research groups have cloned metagenomic fragments downstream of a vector T7 promoter (Lussier et al., 2011; Tegel et al., 2011; Terrón-González et al., 2013), and transformed these metagenomic libraries into $E$. coli screening strains lysogenized with the T7 RNA polymerase from bacteriophage T7 (Studier and Moffatt, 1986). The T7 polymerase is very selective for the $\mathrm{T} 7$ promoter, transcribing the corresponding mRNA at a high frequency and generating longer transcripts, and RNA elongation also occurs at a five-fold faster rate than with the E. coli native RNA polymerase (Tegel et al., 2011). Metagenomic genes cloned in using this method can be transcribed and translated in one of three ways: independent gene transcription and translation, with both promoter and ribosome binding site (rbs) provided by the insert (Figure 6-1A); a transcription fusion with the insert providing its own rbs and translated separately (Figure 6-1B); or expression as a translational fusion with the insert transcribed and translated from the vector promoter and rbs respectively (Figure 6-1C; Gabor et al., 2004). The last method requires the 
insert to be in the same translation open-reading frame (ORF) as the vector start codon, and for no transcription terminators or stop codons to be present in the fragment of DNA before the start of the gene insert (Gabor et al., 2004).

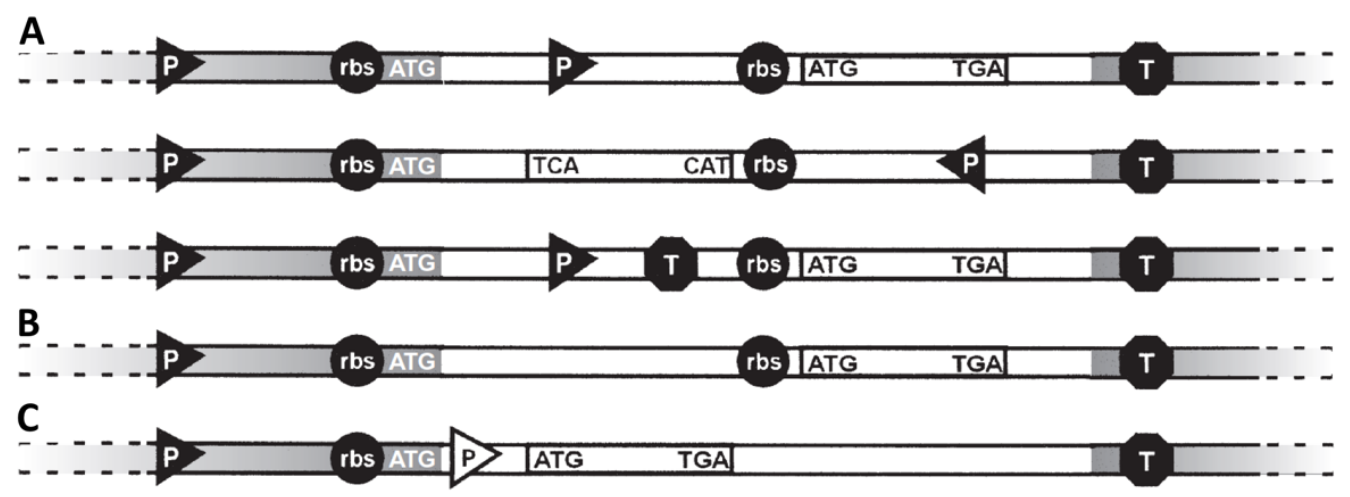

Figure 6-1 Different modes of gene expression utilising a vector T7 promoter in a heterologous bacterial host. Vector DNA is shaded in grey, insert DNA is white. $P$, promoter; $T$, transcription terminator. A) Independent gene expression. The insert can be cloned in either direction. However, if a transcriptional termination sequence is located in-between the present promoter and the coding DNA sequence (cds) start, the gene can only be expressed as a transcriptional fusion (bottom). B) Expression based on a transcriptional fusion. C) Expression dependent on expression signals located on the vector. Here, a promoter that is active in the host system may be present (white triangle). However, a suitable ribosome-binding site (rbs) is lacking, which is why the gene can only be expressed as a fusion protein. ATG and TGA denote the start and stop codons for the cds respectively. Reprinted from Gabor et al. (2004) with permission from Elsevier.

\subsubsection{Previous attempts by the Ackerley lab to recover novel nitroreductase genes}

Some previous research conducted in the Ackerley lab sought to recover novel nitroreductase genes from a metagenomic library constructed from soil taken from a NZ residential property ("the NZ soil library"; Horvat, 2012; Owen et al., 2012). DNA from this soil had been extracted, partially digested with DNaseA, and 1-3kb sized fragments blunt-end cloned into the expression plasmid pETDuet-1 behind the T7 promoter (Owen et al., 2012). To screen for nitroreductases from this library, masked fluorophores were 
utilised, which comprise fluorescence-quenching nitro-substituents attached to an otherwise fluorescent aromatic heterocycle, such that reduction by a nitroreductase allows for detection of the activated fluorophore (Horvat, 2012; Prosser et al., 2013). Transformation of 250,000 clones from this metagenomic DNA library into the E. coli BL21(DE3) strain was followed by incubation with two masked blue fluorophores, and fluorescent cells were selected using FACS (Horvat, 2012). This ultimately led to the discovery of a single nitroreductase that shared $56 \%$ amino acid identity with NfsA_Ec (Horvat, 2012). However, an incredibly high background of other genes recovered (several hundred non-nitroreductases) deemed it likely that this NfsA_Ec homologue had simply been recovered by chance, and that the attempt at FACS selection had not been effective. One likely reason attributed to this was the relatively high levels of cell permeability exhibited by these particular fluorophores that, coupled with low-level expression of cloned genes, may have resulted in the signal-tonoise ratio in the naturally autofluorescent $E$. coli host strain being too low for FACS to work efficiently.

A further attempt conducted in our group at recovering novel nitroreductases through functional screening using niclosamide and nitromasked fluorophores utilised both the NZ soil library and a Swedish soil library as sources of metagenomic DNA (Little, 2015). The Swedish soil library had been created from the DNA extracted from garden compost in Sweden, and contained approximately $1.3 \times 10^{5}$ unique DNA fragments between $2 \mathrm{~kb}-6 \mathrm{~kb}$ in length cloned into the expression plasmid pRSETB (Parachin and Gorwa-Grauslund, 2011). The E. coli 7NT strain (with chromosomal knock-outs of genes encoding for seven endogenous nitroreductases and the TolC multi-drug efflux pump) was lysogenized with $\lambda \mathrm{DE} 3$ (forming the strain 7TL) to allow for the chromosomal integration of the gene for T7 RNA polymerase, as both the Swedish and NZ libraries were cloned into vectors containing T7 promoters (Little, 2015). This was followed by the transformation of both metagenome libraries into this strain and selection of variants that could detoxify $0.5 \mu \mathrm{M}$ niclosamide (Little, 
2015). However counter-screening of 400 of these variants with a nitromasked fluorophore showed no activation of the fluorescent compound, and sequencing revealed a powerful selection of TolC-like efflux pumps rather than nitroreductases (Little, 2015). Unfortunately over the course of this work, contamination of pUCX:KG_NfsA_Ec in the Swedish library (both plasmids are $\operatorname{Kan}^{\mathrm{R}}$ ), and contamination of pUCX:NfsA_Ec in the NZ soil library (both plasmids are $\mathrm{Amp}^{\mathrm{R}}$ ) from other nitroreductase sources in the lab was discovered (Rory Little, personal communication). This contamination was traced back to the original sources of both libraries, and hence for the research described in this chapter the decision was made to create a new metagenomic library to avoid previous contamination issues.

\subsubsection{Functional screening with niclosamide and coloured compounds}

Work presented earlier in this thesis (e.g., Figure 4-5) demonstrates that the ability to detoxify niclosamide is widespread in bacterial nitroreductases. Our previous attempts to utilise niclosamide as a selection compound for metagenomic libraries resulted in a powerful selection for genes encoding TolC efflux pumps over nitroreductases. This suggests that even low-level expression of the former can effectively protect a host cell against niclosamide challenge, whereas high-level nitroreductase expression might be required to achieve the same effect in a tolC deletion host. To overcome this problem, the research described in this chapter utilised a novel strategy to ensure high-level expression of cloned genes from metagenomic DNA. It was also necessary to implement methodology to differentiate between cells that had gained a tolC gene and those that had gained a nitroreductase gene. To specifically target the latter group, nitroaromatic compounds that change colour upon nitro-reduction were considered for use in the counterscreening of niclosamide-detoxifying variants from a metagenomic library.

Previous research conducted by the Ackerley group had shown that two different nitroaromatic compounds, 4(5)-nitroimidazole and 2-methyl-4(5)nitroimidazole (chemical structures shown in Figure 6-2) change colour 
following activation by over-expressed nitroreductases in E. coli - the former to a red-brown compound, and the latter to a blue-green product in growth inhibition assays (Dr Elsie Williams, personal communication). E. coli 7NT colonies over-expressing NfsA_Ec from pUCX could be distinguished from colonies bearing an empty pUCX control on an LB agar plate when a top agarose solution containing $5 \mathrm{mM} 4$-nitroimidazole was poured over mixed colonies (Little, 2015).

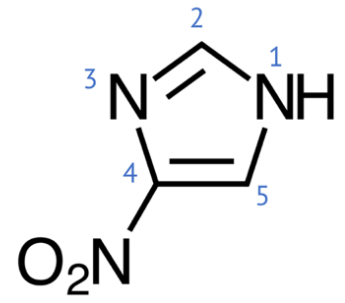

4-nitroimidazole

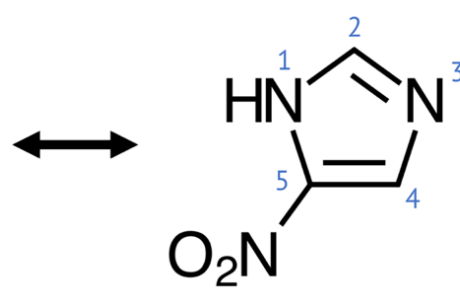

5-nitroimidazole

Figure 6-2 Chemical structures of the annular isomers of 4(5)-nitroimidazole.

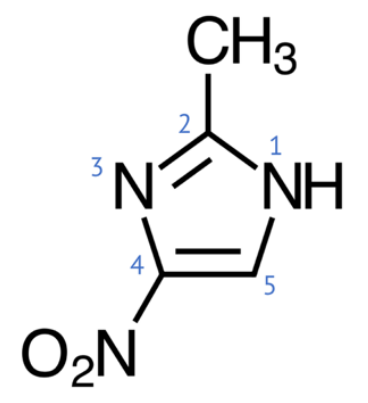

2-methyl-4-nitroimidazole

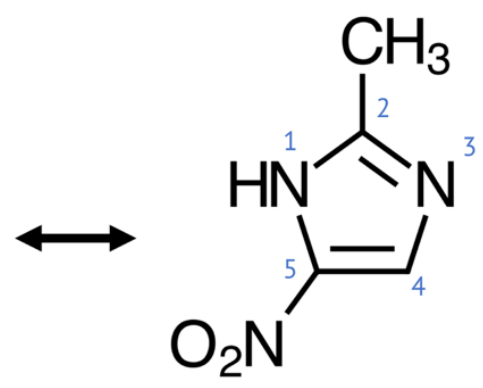

2-methyl-5-nitroimidazole

Figure 6-3 Chemical structures of the annular isomers of 2-methyl-4(5)nitroimidazole.

4(5)-nitroimidazole and 2-methyl-4(5)-nitroimidazole are not prodrugs in their own right, but are intermediates in the synthesis of nitroimidazole drugs (Kraft et al., 1989; Pedada et al., 2013). As shown in Figures 6-2 and 
6-3, the 4(5) notation indicates that both of these compounds exist as annular tautomers, whereby the $\mathrm{H}$ atom can be located on either $\mathrm{N}$ atom in the nitroimidazole ring and thus the position of the nitro group can occur at either the 4- or 5- positions (Feketeová et al., 2015; Miller et al., 1970). However, for the rest of the chapter they will simply be referred to as the 4nitro isomer (4-nitroimidazole and 2-methyl-4-nitroimidazole respectively).

\section{$6.2 \quad$ Aims}

- Evaluate the activity of wild-type nitroreductases from the 58 nitroreductase library with nitro-masked chromophores.

- Create, using a novel cloning strategy, a highly-expressed library containing a T7 promoter.

- Discover nitroreductases in this library through functional selection and screening using nitroaromatic compounds.

- Evaluate the expression of discovered nitroreductases alongside nitroreductases from the 58 nitroreductase library.

\section{$6.3 \quad$ Results}

\subsubsection{Evaluation of the activity of members of the $\mathbf{5 8}$ nitroreductase library} with nitro-masked chromophore compounds

\subsubsection{Growth inhibition $\left(I C_{50}\right)$ assays with nitroreductases and nitro-masked chromophore compounds}

To determine the ability of nitroreductases from the 58 nitroreductase library to activate 4-nitroimidazole or 2-methyl-4-nitroimidazole, the ability of 18 over-expressed nitroreductases in pUCX to sensitise 7NT cells to lethal concentrations of either compound was evaluated (Table 6-1). 


\begin{tabular}{|c|c|c|c|}
\hline Family & Nitroreductase & $\begin{array}{c}\text { 2-methyl-4- } \\
\text { nitroimidazole } \\
\mathrm{IC}_{50}(\mu \mathrm{M})\end{array}$ & $\begin{array}{c}\text { 4- } \\
\text { nitroimidazole } \\
\mathrm{IC}_{50}(\mu \mathrm{M})\end{array}$ \\
\hline NfsA & CO_Frp_Vh & $64 \pm 14$ & $63 \pm 5$ \\
\hline NfsA & NfsA_Vf & $119 \pm 25$ & $123 \pm 14$ \\
\hline NfsA & NfsA_Ck & $139 \pm 30$ & $118 \pm 19$ \\
\hline NfsA & NfsA_Vv & $148 \pm 21$ & $125 \pm 19$ \\
\hline NfsA & NfsA_Ec & $149 \pm 7$ & $113 \pm 27$ \\
\hline NfsA & NfrA_Bs & $209 \pm 23$ & $191 \pm 36$ \\
\hline NfsA & NfsA_St & $215 \pm 45$ & $297 \pm 34$ \\
\hline NfsA & NfsA_Es & $611 \pm 43$ & $671 \pm 69$ \\
\hline NfsA & YcnD_Bs & $646 \pm 146$ & $205 \pm 9$ \\
\hline NfsA & NfsA_Kp & $776 \pm 47$ & $731 \pm 134$ \\
\hline NfsA & EcD_Pp & $1040 \pm 160$ & $>2000$ \\
\hline $\mathrm{NfsB}$ & FRaseI_Vf & $1290 \pm 240$ & $980 \pm 160$ \\
\hline NfsA & NfsA_L̄w & $1440 \pm 360$ & $1710 \pm 240$ \\
\hline NfsA & NfsA_Li & $1640 \pm 270$ & $1910 \pm 30$ \\
\hline $\mathrm{NfsB}$ & YfkO_Bs & $5870 \pm 510$ & $>2000$ \\
\hline NfsA & Frp_Vh & $6230 \pm 1700$ & $>2000$ \\
\hline $\mathrm{NfsB}$ & NfsB_Vh & $7220 \pm 1290$ & $>2000$ \\
\hline $\mathrm{NfsB}$ & NfsB_Es & $8230 \pm 330$ & $>2000$ \\
\hline Nitroreductase 1 & YdgI_Bs & $>10,000$ & $>2000$ \\
\hline & Empty pUCX & >>10,000 & $>>>2000$ \\
\hline
\end{tabular}

Table 6-1 I $C_{50}$ values of 4-nitroimidazole or 2-methyl-4-nitroimidazole mediated growth inhibition of 18 nitroreductases from the 7NT E. coli 58 nitroreductase overexpression library. 2-methyl-4-nitroimidazole or 4-nitroimidazole-dependent growth inhibition was monitored by measuring strain turbidity $\left(O D_{600}\right)$ pre- and post-four hour incubation with a two-fold dilution series of 2-methyl-4nitroimidazole (top concentration of $10 \mathrm{mM}$ ) or 4-nitroimidazole (top concentration of $2 \mathrm{mM}$ ). Percentage growth relative to unchallenged controls was determined and used to calculate the concentration at which 50\% growth inhibition was seen using GraphPad Prism 7.0. Data are the average of 3 biological replicates $\pm S D$. Enzymes are ranked by their ability to sensitise $7 N T$ cells to 2-methyl-4-nitroimidazole. The concentration of 4-nitroimidazole could not be increased above $2 \mathrm{mM}$ due to precipitation of compound in assay media.

Members of the NfsA family were able to sensitise 7NT cells to lower concentrations of 4-nitroimidazole and 2-methyl-4-nitroimidazole than enzymes from the NfsB or Nitroreductase 1 families, suggesting that for functional screening these compounds may be more selective for NfsA enzymes over members of other nitroreductase families (provided sub-lethal concentrations of the compounds were used). The only member of the NfsB family able to sensitise 7NT cells to 4-nitroimidazole was FRaseI_Vf. Due to 
the precipitation of 4-nitroimidazole in assay media at concentrations above $2 \mathrm{mM}, \mathrm{IC}_{50}$ values could be not calculated for all other NfsB family enzymes, EcD_Pp, Frp_Vh or YdgI_Bs.

\subsubsection{Correlation between 4-nitroimidazole or 5-nitroimidazole compound- mediated growth inhibition}

Since the nitro group of 4-nitroimidazole and 2-methyl-4-nitroimidazole compounds can also occur at the 5-nitro position, we sought to determine if a strong correlation between the ability of nitroreductases to activate these two compounds and S33 was present i.e. if nitroreductases exhibiting the most activity with these nitro-masked chromophores were also the most active enzymes with $\mathrm{S} 33$. The $\mathrm{IC}_{50}$ values of $7 \mathrm{NT}$ cells over-expressing 19 different nitroreductases from the 58 nitroreductase library with S33 (from Table 3-4) was correlated with the $\mathrm{IC}_{50}$ values with 4-nitroimidazole or 2methyl-4-nitroimidazole (from Table 6-1). A limited correlation was observed between S33 and 2-methyl-4-nitroimidazole $\left(\mathrm{R}^{2}=0.20\right.$; Figure 6-4), whereas an apparently higher correlation $\left(\mathrm{R}^{2}=0.62\right.$; Figure 6-5) was observed between S33 and 4-nitroimidazole (bearing in mind that seven nitroreductases could not be included in the correlation analysis due to their inability to sensitise E. coli to the highest concentrations of S33 or 4nitroimidazole used). In the case of 2-methyl-4-nitroimidazole, this suggested that metagenomic nitroreductases exhibiting the most activity with the nitro-masked chromophore would not necessarily be highly active with S33. 


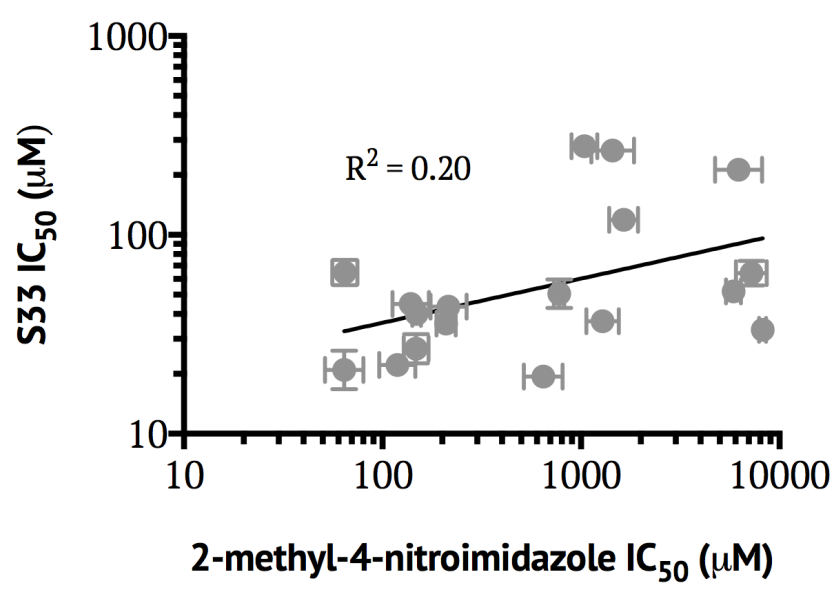

Figure 6-4 Log-log correlation between IC 50 growth inhibitory effects of 2-methyl-4nitroimidazole and S33 in 7NT nitroreductase over-expression strains. $I C_{50}$ values of $7 N T$ cells over-expressing 18 different wild-type nitroreductases from the 58 nitroreductase family following challenge with up to $2 \mathrm{mM}$ 4-nitroimidazole (Table 6-1) or $300 \mu M S 33$ in IC $C_{50}$ assays (Table 3-4). NfSA_Es was dropped from the correlation analysis due to its inability to sensitise 7NT cells to up to $300 \mu M S 33$.

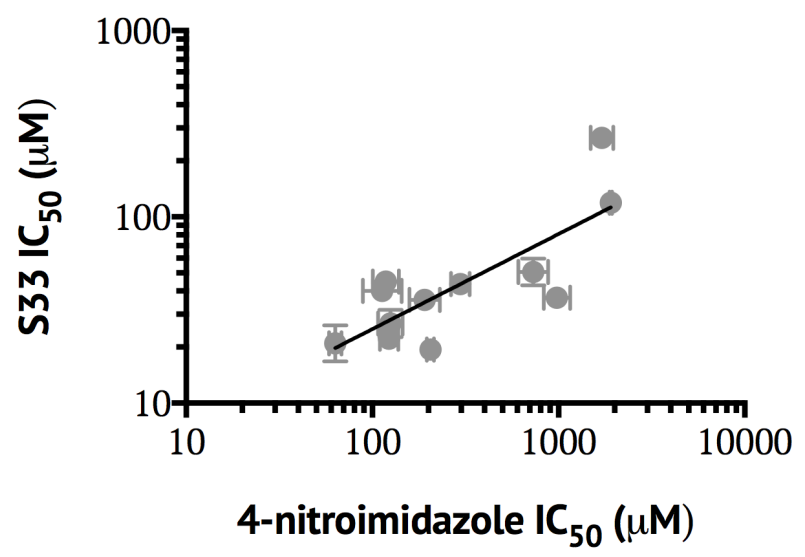

Figure 6-5 Log-log correlation between I $C_{50}$ growth inhibitory effects of 2-methyl-4nitroimidazole and S33 in 7NT nitroreductase over-expression strains. $I C_{50}$ values of $7 N T$ cells over-expressing 18 different wild-type nitroreductases from the 58 nitroreductase family following challenge with up to $2 \mathrm{mM} 4$-nitroimidazole (Table 6-1) or $300 \mu M S 33$ in IC $C_{50}$ assays (Table 3-4). EcD_Pp, YdgI_Bs, $Y f k O \_B s, F r p \_V h, N f s B_{-} V h$, and $N f_{s} B_{-} E s$ were dropped from the correlation analysis due to their inability to sensitise 7NT cells to up to $2 \mathrm{mM} 4$ nitroimidazole. NfSA_Es was dropped from the correlation analysis due to its inability to sensitise 7NT cells to up to $300 \mu M$ S33. 


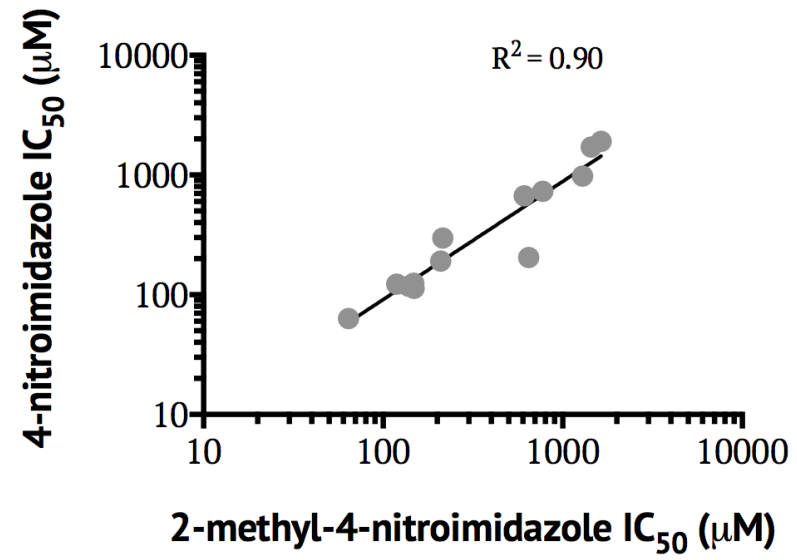

Figure 6-6 Log-log correlation between IC 50 growth inhibitory effects of 4nitroimidazole and 2-methyl-4-nitroimidazole in 7NT nitroreductase overexpression strains. $I C_{50}$ values of $7 N T$ cells over-expressing 18 different wild-type nitroreductases from the 58 nitroreductase family following challenge with up to $2 \mathrm{mM}$ 4-nitroimidazole or $10 \mathrm{mM}$ 2-methyl-4-nitroimidazole in $\mathrm{IC}_{50}$ assays (Table 6-1). EcD_Pp, YdgI_Bs, YfkO_Bs, Frp_Vh, NfsB_Vh, and NfsB_Es were dropped from the correlation analysis due to their inability to sensitise 7NT cells to up to $2 \mathrm{mM} 4$-nitroimidazole.

Conversely, with the exception of EcD_Pp, four NfsB family enzymes and YdgI_Bs (due to the inability to calculate $\mathrm{IC}_{50}$ values of $7 \mathrm{NT}$ over-expressing these enzymes following challenge with 4-nitroimidazole), a strong correlation was observed between the ability of NfsA nitroreductases to sensitise $7 \mathrm{NT}$ cells to 4 -nitroimidazole or 2-methyl-4-nitroimidazole $\left(\mathrm{R}^{2}=\right.$ 0.90; Figure 6-6), suggesting a higher likelihood of recovering NfsA family enzymes following counter-screening with these compounds than enzymes from the NfsB or Nitroreductase 1 families.

\subsubsection{Attempts to create a metagenomic library}

\subsubsection{Novel methods for cloning of metagenomic DNA libraries}

As discussed in Section 6.1.3.1, previous attempts by our lab to utilise 0.5 $\mu \mathrm{M}$ niclosamide as a positive selection compound for nitroreductases from metagenomic libraries resulted only in the selection of tolC-like efflux pump genes. As noted above, we hypothesised that the expression of 
nitroreductase genes was too low to allow for successful detection of nitroreductase enzymes. We therefore implemented an alternative cloning strategy that could allow for the insertion of genes directly after the T7 promoter and vector rbs, to maximise their expression.

The restriction enzyme FatI recognises the nucleotide sequence CATG, and DNA fragments digested with this enzyme can be ligated into a vector digested with NcoI, as they would share compatible cohesive ends (Figure 6-7).

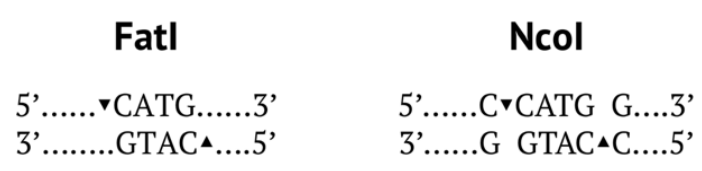

Figure 6-7 Fatl and Ncol restriction enzyme DNA cleavage sites.

We reasoned that the use of FatI for partial cleavage of a metagenomic library could allow for the cloning of genes having an ATG start codon immediately preceded by a $\mathrm{C}$, into a vector digested with NcoI. To create such a vector a modified multiple-cloning site of pET28a+ was designed, within which the NdeI restriction site occurring after the $\mathrm{His}_{6}$-tag was replaced with an NcoI site, and all other NcoI sites deleted from the MCS (Figure 6-8). Genes cloned in using this method would not require their own transcription promoter or rbs to be encoded upstream, as they would be transcribed from the $\mathrm{T} 7$ promoter and translated from the vector rbs, in frame with the $\mathrm{His}_{6}$-tag. 


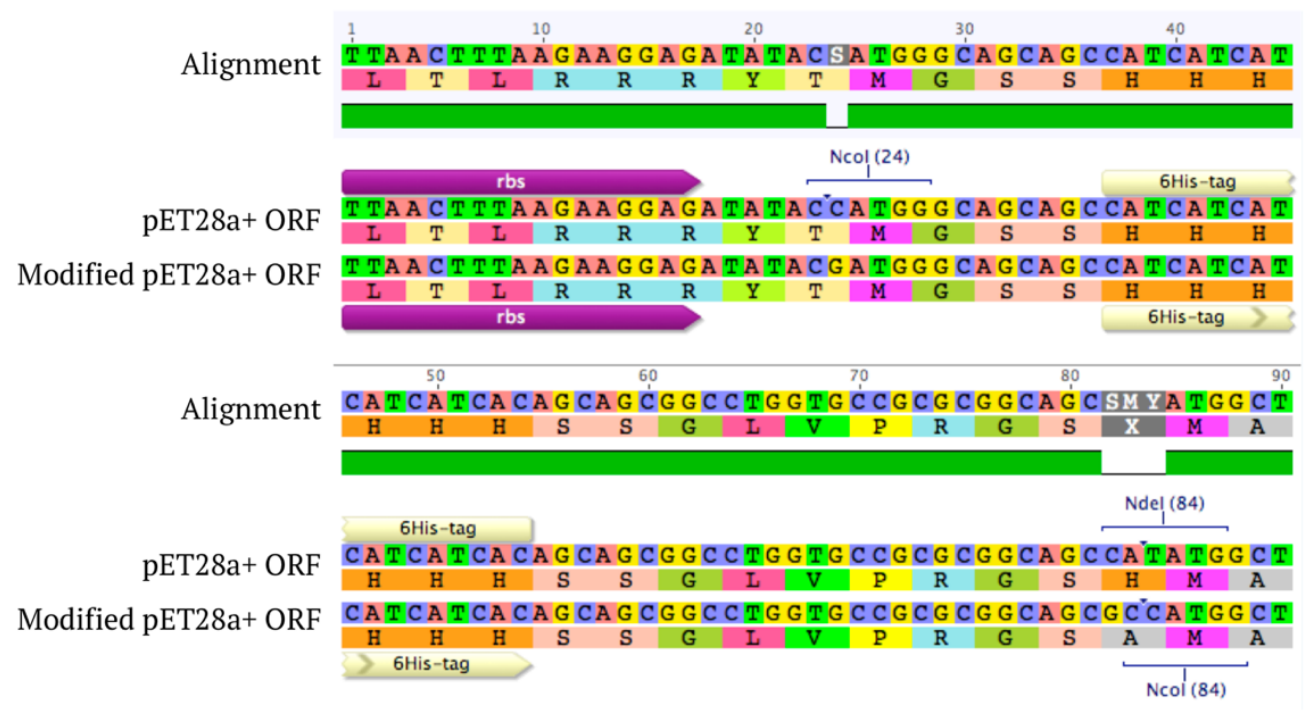

Figure 6-8 Modified pET28a+ multiple-cloning site for expression of metagenomic fragments.

This modified multiple-cloning site was cloned into an E. coli/Pseudomonas shuttle vector, pUCP22 (West et al., 1994), which contained a $g^{R}$ (gentamicin resistance) gene, as this would presumably reduce the risk of contamination of the metagenomic library with other nitroreductase sources in the Ackerley lab.

Our first attempt at creating a metagenomic library utilised ZooDoo compost as a source of DNA (manure derived from mammals from Wellington Zoo that had been used to create compost and mixed with bark). We reasoned that this would be a rich source of gastrointestinal bacterial species, however the approach failed due to extraction of only degraded, low-molecular-weight DNA.

Cosmid libraries that had been previously made from NZ soil samples by members of the Owen lab were then investigated as another potential source of DNA. This DNA was of a high molecular weight, free from organics such as humic acid that can reduce cloning efficiency, and not degraded. We considered that the inevitable cloning of portions of the cosmid vector into the library would have little effect on subsequent screening of the library. However, following the cloning of this library we noted issues with the pET- 
pUCP22 plasmid construct (unexpected upregulation of plasmid copy number exerting confounding effects on colony size and colour). Moreover, post niclosamide selection there was an exceptionally high rate of false positives deriving from fragments of inserted cosmid vector DNA (variants that could detoxify niclosamide but contained no ORFs within the inserted fragment, and exhibited no nitroreductase activity when counter-screened with nitro-masked chromophores or metronidazole), which we were unable to explain. These observations led to the abandonment of both the cosmid DNA and the pET-pUCP22 construct.

Due to time constraints towards the end of this research, rather than conducting a comprehensive metagenomic screen on a de novo library, a proof-of-principle library was created instead in the pET28a+ vector. This library utilised a small collection of genomic DNA from several culturable bacterial strains in the Ackerley lab that were known to contain nitroreductase genes with FatI restriction sites preceding the gene start codons. We reasoned that this would allow comprehensive testing of whether this cloning strategy was generally feasible and effective, and could improve the proportion of nitroreductase to tolC genes discovered.

\subsubsection{Creation of multi-genomic DNA library in pET28a+}

\subsubsection{Selection of bacterial genomes with Fatl-cleavable nitroreductases}

For the selection of bacterial genomes containing nitroreductase genes with a FatI restriction site immediately preceding the ATG start codon, we first identified suitable candidates through the analysis of published genomic sequences of bacterial strains available in the Ackerley lab, using annotations provided by the NCBI Prokaryotic Genome Annotation Pipeline (https://www.ncbi.nlm.nih.gov/genome/annotation prok/). Three strains, $B$. subtilis, C. koseri, and P. putida were found to contain at least one member from the NfsA or NfsB enzyme families (Table 6-2), and had previously been utilised as sources of enzymes for the 58 nitroreductase library (Prosser et al., 2013). The fourth strain, Burkholderia thailandensis, was a culturable soil 
bacterium utilised by the Owen lab at Victoria University and made available to us for this research. This strain contained putative nitroreductase genes from three different families within the Nitro FMN superfamily (Table 6-2), one of which (the Nitroreductase 2 family) had no homologues within the current 58 nitroreductase library.

\begin{tabular}{|c|c|c|c|c|}
\hline Nitroreductase & $\begin{array}{c}\text { Bacterial } \\
\text { strain }\end{array}$ & Family & $\begin{array}{c}\text { Accession } \\
\text { number }\end{array}$ & $\begin{array}{l}\text { Number of } \\
\text { internal Fatl } \\
\text { restriction } \\
\text { sites }\end{array}$ \\
\hline YfkO_Bs & $\begin{array}{l}\text { B. subtilis } \\
\text { str. } 168\end{array}$ & NfsB & $\frac{\text { ADE73858 }}{.1}$ & 3 \\
\hline YdgI_Bs & $\begin{array}{l}\text { B. subtilis } \\
\text { str. } 168\end{array}$ & $\begin{array}{c}\text { Nitroreductase } \\
1\end{array}$ & $\frac{\text { WP } 00322}{\underline{5379.1}}$ & 5 \\
\hline NfsA_Ck & $\begin{array}{c}\text { C. koseri } \\
\text { ATCC } 27156\end{array}$ & NfsA & $\frac{\mathrm{WP} 01213}{\underline{3106.1}}$ & 3 \\
\hline NfsA_Pp & $\begin{array}{c}\text { P. putida } \\
\text { KT2440 }\end{array}$ & NfsA & $\frac{\text { WP 01095 }}{\underline{3425.1}}$ & 2 \\
\hline NfsB_Pp & $\begin{array}{l}\text { P. putida } \\
\text { KT2440 }\end{array}$ & $\mathrm{NfsB}$ & $\frac{\text { WP } 01095}{\underline{3384.1}}$ & 1 \\
\hline AzoR_Pp & $\begin{array}{l}\text { P. putida } \\
\text { KT2440 }\end{array}$ & AzoR & $\frac{\mathrm{ABQ} 77513}{\underline{.1}}$ & 5 \\
\hline $\begin{array}{l}\text { Uncharacterised } \\
\text { nitroreductase }\end{array}$ & $\begin{array}{c}\text { P. putida } \\
\text { KT2440 }\end{array}$ & $\begin{array}{c}\text { Nitroreductase } \\
2\end{array}$ & $\frac{\text { WP } 01095}{\underline{5256.1}}$ & 2 \\
\hline $\begin{array}{l}\text { Uncharacterised } \\
\text { nitroreductase }\end{array}$ & $\begin{array}{c}\text { B. } \\
\text { thailandensis } \\
\text { E264 }\end{array}$ & $\begin{array}{c}\text { Nitroreductase } \\
2\end{array}$ & $\frac{\text { WP } 00988}{\underline{8637.1}}$ & 1 \\
\hline $\begin{array}{c}\text { Uncharacterised } \\
\text { nitroreductase }\end{array}$ & $\begin{array}{c}\text { B. } \\
\text { thailandensis } \\
\text { E264 }\end{array}$ & $\begin{array}{l}\text { Arsenite } \\
\text { oxidase }\end{array}$ & $\frac{\text { WP 00989 }}{\underline{3809.1}}$ & 1 \\
\hline $\begin{array}{l}\text { Uncharacterised } \\
\text { nitroreductase }\end{array}$ & $\begin{array}{c}\text { B. } \\
\text { thailandensis } \\
\text { E264 }\end{array}$ & $\begin{array}{c}\text { Nitroreductase } \\
5\end{array}$ & $\frac{\text { WP } 00989}{\underline{7344.1}}$ & 3 \\
\hline
\end{tabular}

Table 6-2 List of candidate nitroreductases containing a Fatl restriction site immediately preceding the ATG start codon.

\subsubsection{Screening of control nitroreductases with 4-nitroimidazole and 2-methyl- 4-nitroimidazole}

As the genes $y f k O_{-} B s, y d g I_{-} B s, n f s A_{-} C k, n f s_{-} P p, n f s B_{-} P p$, and $a z o R_{-} P p$ had already been cloned into pET28a+ (albeit with a preceding His $_{6}$-tag), they were transformed into the 7TL strain to observe the ability of the encoded 
$\mathrm{His}_{6}$-tagged enzymes to detoxify $0.5 \mu \mathrm{M}$ niclosamide and reduce 4nitroimidazole or 2-methyl-4-nitroimidazole on LB agar plates containing $50 \mu \mathrm{M}$ IPTG. Enzymes were first plated on LB agar plates containing niclosamide and incubated at $37^{\circ} \mathrm{C}$ overnight. The following day a top agarose solution containing $5 \mathrm{mM} 4$-nitroimidazole or $10 \mathrm{mM}$ 2-methyl-4nitroimidazole was poured over the colonies, and the plate left at room temperature for up to 24 hours for colour changes to be observed.

\begin{tabular}{|c|c|c|c|}
\hline Nitroreductase & $\begin{array}{c}\text { Percentage of } \\
\text { colonies able to } \\
\text { detoxify } 0.5 \mu \mathrm{M} \\
\text { niclosamide }\end{array}$ & $\begin{array}{l}\text { Time at which } \\
\text { colour change } \\
\text { with } 5 \mathrm{mM} \mathrm{4-} \\
\text { nitroimidazole in } \\
\text { top agarose was } \\
\text { observable }\end{array}$ & $\begin{array}{c}\text { Time at which } \\
\text { activity with } 10 \\
\text { mM 2-methyl-4- } \\
\text { nitroimidazole in } \\
\text { top agarose was } \\
\text { observable }\end{array}$ \\
\hline YfkO_Bs & $100 \%$ & 1 hour & 1 hour \\
\hline YdgI_Bs & $70 \%$ & 4 hours & 1 hour \\
\hline NfsA_Ck & $40 \%$ & 1 hour & 1 hour \\
\hline NfsA_Pp & $70 \%$ & 1 hour & 1 hour \\
\hline NfsB_Pp & $10 \%$ & 24 hours & 24 hours \\
\hline AzoR_Pp & $100 \%$ & No activity & No activity \\
\hline $\begin{array}{c}\text { Empty } \\
\text { pET28a+ } \\
\text { plasmid }\end{array}$ & $0 \%$ & No activity & No activity \\
\hline
\end{tabular}

Table 6-3 Activity of control nitroreductases in pET28a+ with niclosamide and nitro-masked chromophores. The percentage of 7NT cells over-expressing six different nitroreductases on LB agar plates containing $50 \mu M$ IPTG and $0.5 \mu M$ niclosamide was calculated relative to growth on plates without niclosamide. Activities of the same nitroreductases with the nitro-masked chromophores 4nitroimidazole or 2-methyl-4-nitroimidazole were determined as the time taken (in hours) for a colour change of these same colonies to be observed following top agarose administration of either compound.

Of the six control nitroreductases tested, only 7NT cells over-expressing YfkO_Bs or AzoR_Pp showed no inhibition in their ability to form colonies on $0.5 \mu \mathrm{M}$ niclosamide (Table 6-3). YdgI_Bs, NfsA_Ck and NfsA_Pp showed varying levels of growth inhibition, and only $10 \%$ of NfsB_Pp overexpressing cells were able to detoxify this concentration of niclosamide. This suggested that selection of a metagenomic library on niclosamide might miss some nitroreductase-containing colonies, and in an attempt to 
alleviate this issue we attempted to use lower concentrations of niclosamide, or upregulate nitroreductase expression through increased concentrations of IPTG in the agar plate. Unfortunately, 7NT cells bearing an empty pET28a+ plasmid were able to grow on plates containing $0.25 \mu \mathrm{M}$ niclosamide, and increased IPTG concentrations (up to $250 \mu \mathrm{M}$ ) negatively affected the growth of nitroreductase over-expressing cells (results not shown).

Nevertheless, the use of nitro-masked chromophore-containing top agarose was able to distinguish between $7 \mathrm{NT}$ cells over-expressing active nitroreductases from those bearing inactive nitroreductases (AzoR_Pp, which does not exhibit any activity with S33; Figure 3-11) or an empty pET28a+ control (Table 6-3). Surprisingly, nitroreductases such as YdgI_Bs, for which $\mathrm{IC}_{50}$ values with either nitro-masked chromophore could not be determined (Table 6-1), did exhibit activity with these compounds upon their inclusion in the top agarose solution (Table 6-3).

\subsubsection{Creation of multi-genomic DNA library}

For the creation of the proof-of-principle FatI library from bacterial gDNA, genomic DNA was extracted from strains of P. putida, C. koseri, B. subtilis and $B$. thailandensis that had each been cultured overnight in $100 \mathrm{~mL} \mathrm{LB}, 37$ ${ }^{\circ} \mathrm{C}$ at $200 \mathrm{rpm}$. They were mixed in equal molar ratios (relative to the size of respective chromosomal DNA sequences) and a test restriction digest was performed using $300 \mathrm{ng}$ multi-genomic DNA and varying concentrations of FatI (Figure 6-9) to determine the optimal concentration for a partial restriction digest.

$0.25 \mathrm{U}$ FatI gave the highest concentration of digested DNA at the desired size i.e. 1-2.5 kB (Figure 6-9, Lane 3), and this reaction was scaled up to allow for the digestion of $50 \mu \mathrm{g}$ multi-genomic DNA. The entire restriction digest was visualised on a DNA agarose gel containing SyBr Safe by gel electrophoresis, and the segment of gel containing the 1-2.5 kb fragments of DNA was dissected and the DNA electro-eluted from the agarose gel. The DNA was ligated into NcoI-cleaved pET28a+ (note: the vector was not 
treated with alkaline phosophatase prior to ligation to prevent self-ligation, as we had previously observed extremely low numbers of transformations following this step), resulting in a $30 \%$ insert rate. This ligation was first transformed into the E. coli strain EC100 (i.e. DH10B, a specialized transformation strain; Durfee et al., 2008) and variants were recovered as per Section 2.6.9.5, plasmid DNA extracted and transformed into the E. coli 7TL screening strain. In total 5 million unique variants were transformed into the 7TL strain.

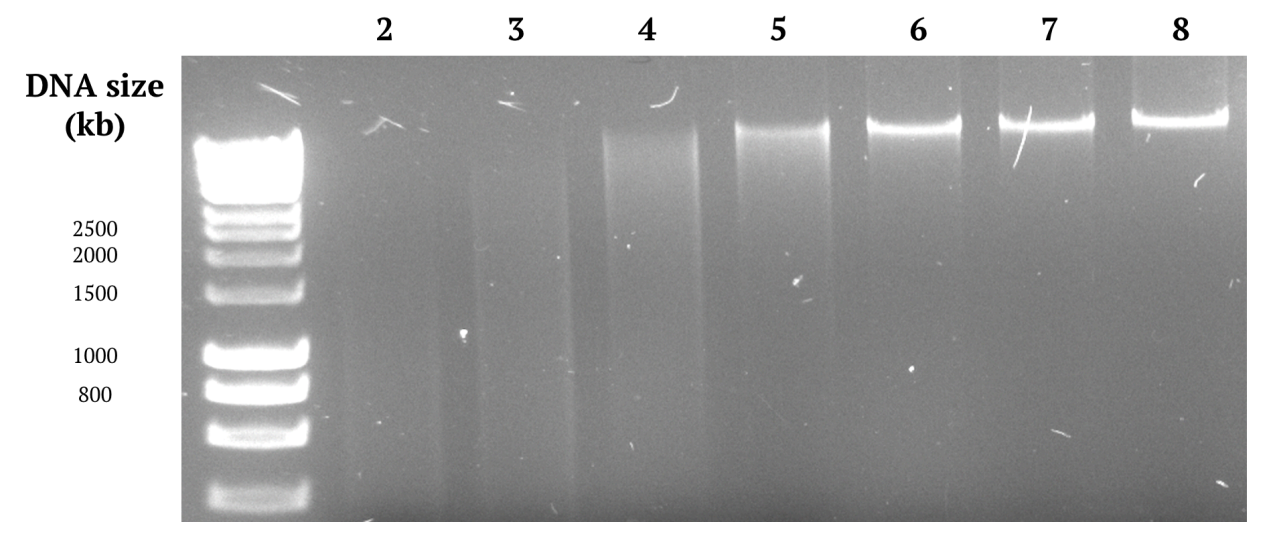

Figure 6-9 Partial restriction enzyme digest of multi-genomic DNA with Fatl. Cleavage of $300 \mathrm{ng}$ of mixed genomic DNA across two-fold dilutions of FatI. (2) $0.5 \mathrm{U}$; (3) $0.25 \mathrm{U}$; (4) $0.13 \mathrm{U}$; (5) $0.063 \mathrm{U}$; (6) $0.032 \mathrm{U}$; (7) 0.016 U FatI; (8) undigested control.

\subsubsection{Screening of multi-genomic library with niclosamide}

The metagenomic library in 7TL was plated on LB agar plates containing 50 $\mu \mathrm{M}$ IPTG and $0.5 \mu \mathrm{M}$ niclosamide. Around $0.015 \%$ of variants were able to detoxify niclosamide (roughly 7,500 colonies of the 5 million total), and of these, 72 clones were picked and sequenced to determine the number of variants containing tolC-like genes vs nitroreductase genes (Table 6-4). 


\begin{tabular}{|c|c|c|c|c|c|c|}
\hline Protein & Type & Bacteria & $\begin{array}{l}\text { Cloned position (forward/reverse transcription } \\
\text { frame, position of start codon) }\end{array}$ & $\begin{array}{c}\# \\
\text { Clones }\end{array}$ & $\begin{array}{c}\% \text { of } \\
\text { total } \\
\text { clones }\end{array}$ & Accession \\
\hline TolC & TolC & C. koseri & $\begin{array}{l}\text { Forward, starts } 127 \text { bp downstream from NcoI } \\
\text { Forward, starts } 582 \text { bp downstream from NcoI } \\
\text { Reverse }\end{array}$ & $\begin{array}{c}2 \\
1 \\
18\end{array}$ & 29 & $\frac{\text { WP } 012135}{\underline{166.1}}$ \\
\hline TolC & TolC & $\begin{array}{l}\text { E. coli } \\
\text { W3110 }\end{array}$ & Reverse & 22 & 31 & $\frac{\mathrm{ACM} 48572 .}{1}$ \\
\hline NfsA_Ec & NfsA & $\begin{array}{l}\text { E. coli } \\
\text { W3110 }\end{array}$ & $\begin{array}{l}\text { Forward, starts } 106 \mathrm{bp} \text { downstream from NcoI } \\
\text { Forward, starts } 182 \mathrm{bp} \text { downstream from NcoI }\end{array}$ & $\begin{array}{c}10 \\
1\end{array}$ & 15 & $\underline{\mathrm{APC} 51123.1}$ \\
\hline AzoR_Ec & AzoR & $\begin{array}{l}\text { E. coli } \\
\text { W3110 }\end{array}$ & $\begin{array}{c}\text { Forward, start codon encoded for by NcoI } \\
\text { Forward, starts } 587 \text { bp downstream from NcoI }\end{array}$ & $\begin{array}{l}6 \\
1\end{array}$ & 10 & $\frac{\text { WP } 000048}{950.1}$ \\
\hline NfsB_Ec & $\mathrm{NfsB}$ & $\begin{array}{l}\text { E. coli } \\
\text { W3110 }\end{array}$ & $\begin{array}{c}\text { Forward, starts } 11 \text { bp downstream from NcoI } \\
\text { Reverse }\end{array}$ & $\begin{array}{l}1 \\
1\end{array}$ & 3 & $\frac{\mathrm{COR} 80176 .}{1}$ \\
\hline YodC & $\begin{array}{c}\text { Nitroreductase } \\
1\end{array}$ & B. subtilis & $\begin{array}{l}\text { Forward, starts } 102 \text { bp downstream from NcoI } \\
\text { Reverse }\end{array}$ & $\begin{array}{l}2 \\
5\end{array}$ & 10 & $\frac{\text { WP } 003231}{196.1}$ \\
\hline NfsA_Ck & NfsA & C. koseri & Forward, start codon encoded for by NcoI & 1 & 1 & $\frac{\mathrm{WP} 012133}{106.1}$ \\
\hline NfsB_Ck & $\mathrm{NfsB}$ & C. koseri & Reverse & 1 & 1 & $\frac{\text { WP } 047458}{272.1}$ \\
\hline
\end{tabular}

Table 6-4 Sequencing results of metagenomic hits that could detoxify $0.5 \mu \mathrm{M}$ niclosamide. 
The sequencing results showed an extremely high proportion of genes from the $E$. coli strain W3110 (tolC, $n f f_{A_{-}} E c, a z o R_{-} E c, n f B_{-} E c$ ) cloned into the multi-genomic library, which was surprising as this strain had not been utilised as a source of genomic DNA due to the lack of nitroreductases containing a FatI restriction site preceding the enzyme start codon. Ultimately it turned out that the lab stock of $P$. putida KT2440 was contaminated with E. coli W3110, and the high incidence of these genes being found may have been exacerbated by the usage of the 7TL strain for screening, which is derived from W3110. In total, tolC genes only made up 59\% of all multi-genomic hits discovered, with half derived from E. coli W3110 and half from C. koseri (the C. koseri TolC protein shares 91\% protein identity with E. coli TolC). E. coli W3110 nitroreductases made up 28\% of proteins discovered (NfsA_Ec, NfsB_Ec, AzoR_Ec), and the YodC nitroreductase from $B$. subtilis, which is not present in the 58 nitroreductase family, was also found in $10 \%$ of variants. The only other nitroreductases found were NfsA_Ck and NfsB_Ck from C. koseri. The majority of genes discovered were not cloned in using the FatI strategy (only $n f f_{S} A_{-} C k$ and $a z o R_{-} E c$ genes had their start codons encoded within the NcoI restriction site, i.e. Figure 6-1 C), however they were cloned in behind the T7 promoter, suggesting that they could be transcribed by the T7 promoter (and translated not through the rbs located on pET28a+, but through rbs sequences present in the cloned DNA fragment; i.e. Figure 6-1 B). Seven variants (containing genes encoding for YodC, NfsB_Ec or NfsB_Ck) were cloned in the reverse direction to the $\mathrm{T} 7$ promoter, suggesting that these genes were being expressed from their own transcription and rbs translation sequences within the cloned DNA fragments (i.e. Figure 6-1 A).

\subsubsection{Counter-screening of niclosamide-selected colonies with 4-nitroimidazole and 2-methyl-4-nitroimidazole}

In parallel with the experiments described in Section 6.3.3.4, variants from the multi-genomic library that could detoxify $0.5 \mu \mathrm{M}$ niclosamide were 
counter-screened with top agarose containing $5 \mathrm{mM}$ 4-nitroimidazole (Figure 6-10A) or $10 \mathrm{mM}$ 2-methyl-4-nitroimidazole (Figure 6-10B) in an attempt to distinguish nitroreductase genes from tolC genes. 81 colonies that could activate either compound were also picked and sequenced (Table 6-5).

A

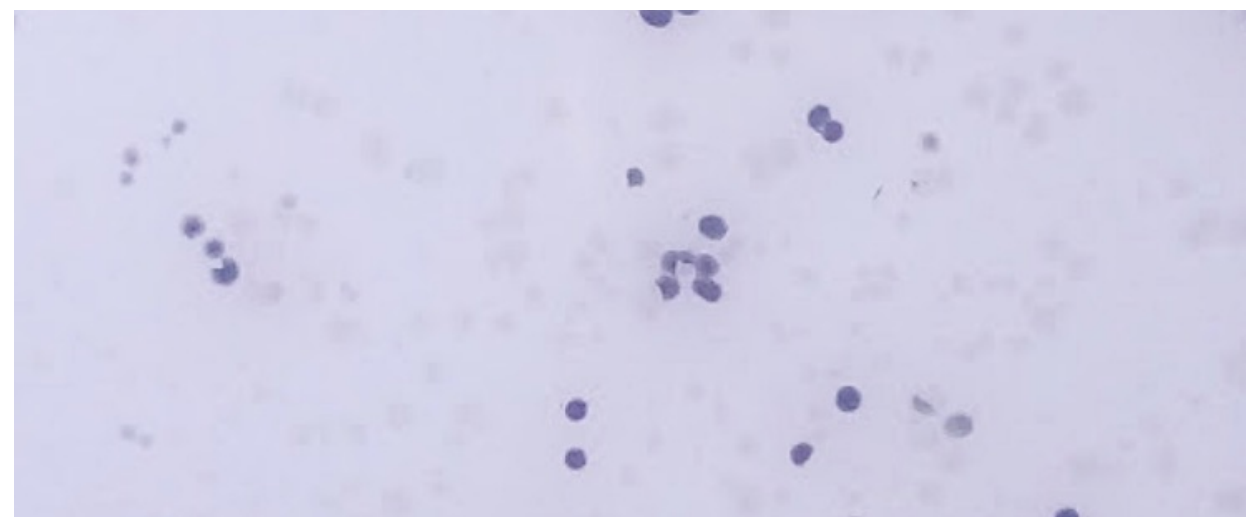

B

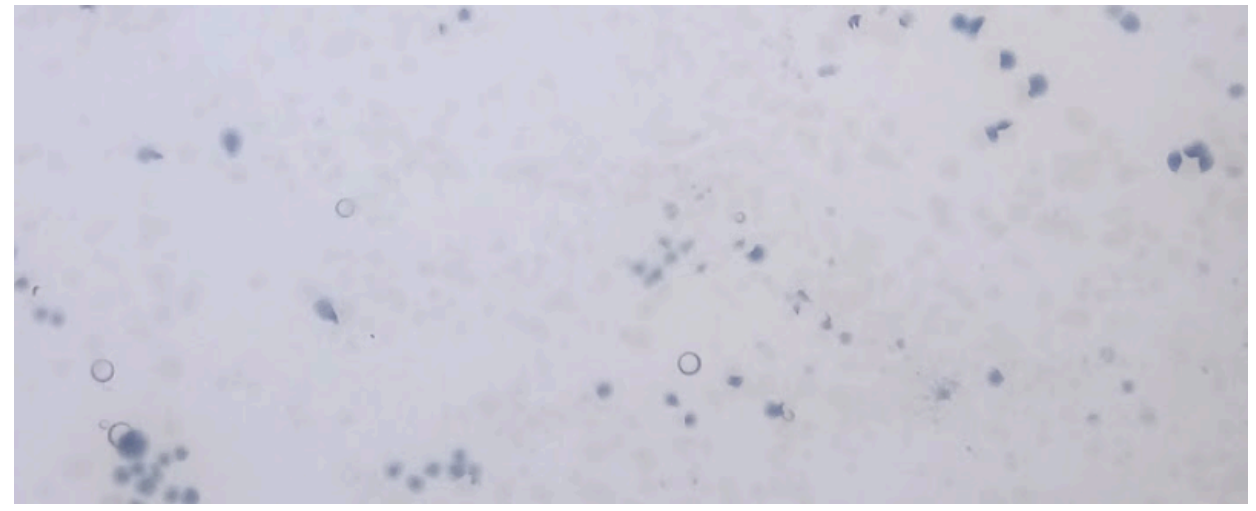

Figure 6-10 Activation of nitro-masked chromophores by multi-genomic library variants also capable of detoxifying $0.5 \mu \mathrm{M}$ niclosamide. $7 N T$ cells over-expressing variants from the multi-genomic library that were able to form colonies on $L B$ agar plates containing $0.5 \mu M$ niclosamide were counter-screened with top agarose containing A) 5 mM 4-nitroimidazole; or B) 10 mM 2-methyl-5nitroimidazole. 


\begin{tabular}{|c|c|c|c|c|c|c|}
\hline Protein & Type & Bacteria & $\begin{array}{l}\text { Cloned position (forward/reverse transcription } \\
\text { frame, position of start codon) }\end{array}$ & $\begin{array}{c}\# \\
\text { Clones }\end{array}$ & $\begin{array}{l}\% \text { of } \\
\text { total } \\
\text { clones }\end{array}$ & Accession \\
\hline NfsA_Ec & NfsA & $\begin{array}{l}\text { E. coli } \\
\text { W3110 }\end{array}$ & $\begin{array}{l}\text { Forward, starts } 106 \text { bp downstream from NcoI } \\
\text { Forward, starts } 182 \text { bp downstream from NcoI } \\
\text { Forward, starts } 630 \text { bp downstream from NcoI } \\
\text { Forward, starts } 782 \text { bp downstream from NcoI } \\
\text { Reverse }\end{array}$ & $\begin{array}{c}60 \\
1 \\
1 \\
1 \\
3\end{array}$ & 81 & $\underline{\text { APC51123.1 }}$ \\
\hline NfsB_Ec & NfsB & $\begin{array}{l}\text { E. coli } \\
\mathrm{W} 3110\end{array}$ & $\begin{array}{c}\text { Forward, starts } 11 \text { bp downstream from NcoI } \\
\text { Forward, starts } 234 \text { bp downstream from NcoI } \\
\text { Reverse }\end{array}$ & $\begin{array}{l}2 \\
2 \\
2\end{array}$ & 7 & $\underline{\text { CQR80176.1 }}$ \\
\hline NfsA_Ck & NfsA & C. koseri & $\begin{array}{l}\text { Forward, start codon encoded for by NcoI } \\
\text { Forward, starts } 259 \text { bp downstream from NcoI } \\
\text { Forward, starts } 511 \text { bp downstream from NcoI }\end{array}$ & $\begin{array}{l}2 \\
1 \\
1\end{array}$ & 5 & WP 012133106.1 \\
\hline NfsB_Ck & NfsB & C. koseri & $\begin{array}{c}\text { Forward, starts } 261 \text { bp downstream from NcoI } \\
\text { Reverse }\end{array}$ & $\begin{array}{l}1 \\
1\end{array}$ & 2 & WP 047458272.1 \\
\hline NfsB_Pp & NfsB & P. putida & Forward, start codon encoded for by NcoI & 1 & 1 & WP 010953384.1 \\
\hline YodC & $\begin{array}{c}\text { Nitroreductase } \\
1\end{array}$ & $\begin{array}{c}\text { B. } \\
\text { subtilis }\end{array}$ & Forward, starts $99 \mathrm{bp}$ downstream from NcoI & 1 & 1 & WP 003231196.1 \\
\hline
\end{tabular}

Table 6-5 Sequencing results of $0.5 \mu \mathrm{M}$ niclosamide-selected metagenomic hits that could reduce either 4-nitroimidazole or 2-methyl-4nitroimidazole. 
As expected, no genomic variants containing tolC genes were found following counter-screening with either nitro-masked chromophore. The proportion of genomic fragments expressing either NfsA_Ec or NfsB_Ec from E. coli W3110 increased substantially, with NfsA_Ec now making up 81\% of all counter-screened variants, and NfsB_Ec expressed in 9\% of variants. AzoR_Ec, which does not exhibit activity with either 4-nitroimidazole or 2methyl-4-nitroimidazole (Table 6-3) was not present in any counterscreened variants. The incidence of YodC from B. subtilis dropped from 9\% (Table 6-4) to 1\% following counter-screening (Table 6-5; though it does exhibit some activity with both nitro-masked fluorophores; results not shown), however the occurrences of both NfsA_Ck and NfsB_Ck increased (from 1\% each in Table 6-4 to 5\% and 2\% respectively; Table 6-5). Counterscreening also revealed one variant over-expressing NfsB_Pp from P. putida, which had not been found following niclosamide selection alone. The counter-screening of niclosamide-detoxifying variants with nitro-masked chromophores therefore appeared to be a successful means of selecting solely for nitroreductase variants, with the caveat that certain nitroreductases (AzoR_Ec) that do not exhibit activity with the masked chromophores will be lost from the pool of nitroreductases recovered.

\subsubsection{Expression levels of nitroreductase hits from multi-genomic library}

To determine the relative levels of expression of nitroreductase enzymes found in Sections 6.3.3.4 and 6.3.3.5, SDS-PAGE analysis of these variants was conducted. Each variant was grown in two separate cultures at $37^{\circ} \mathrm{C}$, $200 \mathrm{rpm}$ for 4 hours, with one culture containing the addition of $50 \mu \mathrm{M}$ IPTG to determine if the gene was being transcribed from the vector $\mathrm{T} 7$ promoter (Figure 6-11). Protein levels in whole-cell lysate were compared to that of their $\mathrm{His}_{6}$-tagged counter-parts where available.

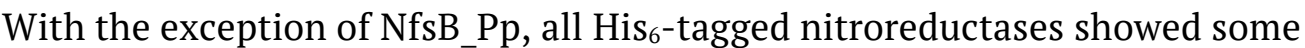
basal protein expression (Figure 6-11, Samples 1,7,11,15) that was substantially upregulated upon IPTG induction. Three of the four NfsA_Ec 
variants cloned in downstream of the T7 promoter (Figure 6-11, Samples 2, 4-5) showed upregulation of protein expression following IPTG induction, suggesting transcription from the T7 promoter even though all samples were cloned in substantially downstream from the NcoI restriction site. Sample 6, which contains NfsA_Ec cloned in the reverse frame, showed no appreciable basal protein expression and no up-regulation following induction with IPTG. None of the NfsB_Ec samples showed basal protein expression or IPTG-induced up-regulation (Figure 6-11, Samples 8-10), suggesting that only minimal expression of this nitroreductase was required for activity with niclosamide and nitro-masked chromophores. Surprisingly, both the nitroreductases NfsA_Ck and NfsB_Pp, when cloned in such that their start codon was encoded for by NcoI (Figure 6-11, Samples 12, 19) exhibited minimal basal protein expression and no up-regulation in expression following IPTG induction. This suggested that perhaps that the presence of a C-terminal His $_{6}$-tag (at least in the case of NfsA_Ck) aided in the expression of high levels of soluble protein. No observable expression was seen in any other NfsA_Ck or NfsB_Ck variants, regardless of whether they were cloned in downstream of the T7 promoter (Figure 6-11, Samples 13-14,16) or in the reverse frame (Figure 6-11 Sample 17). A slight upregulation of YodC from B. subtilis cloned in downstream of the Figure 6-11, Sample 20), and no visible expression was observed of the same nitroreductase cloned in the reverse frame (Figure 6-11, Sample 21). 
- IPTG

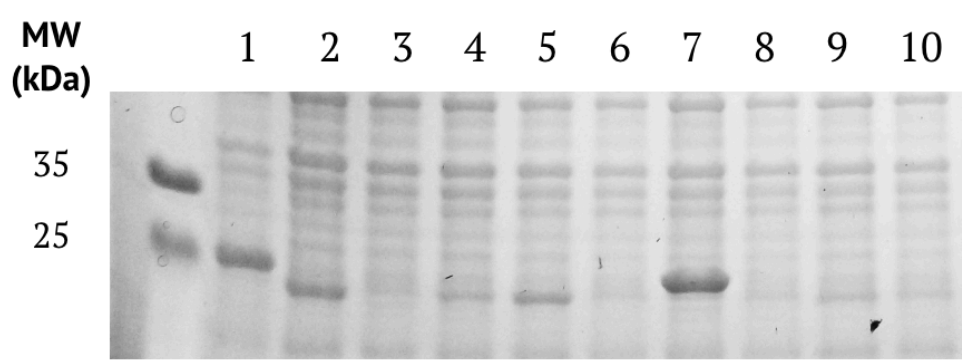

- IPTG

$\begin{array}{llllllllllll}\text { MW } & 11 & 12 & 13 & 14 & 15 & 16 & 17 & 18 & 19 & 20 & 21\end{array}$

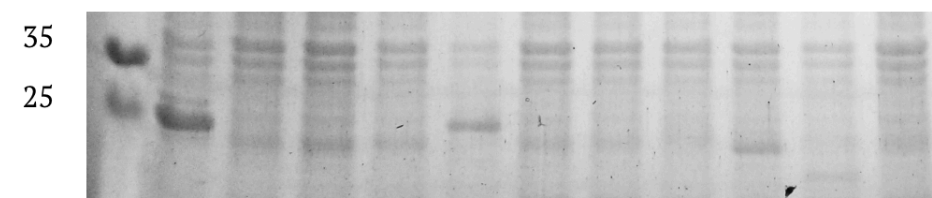

+ IPTG

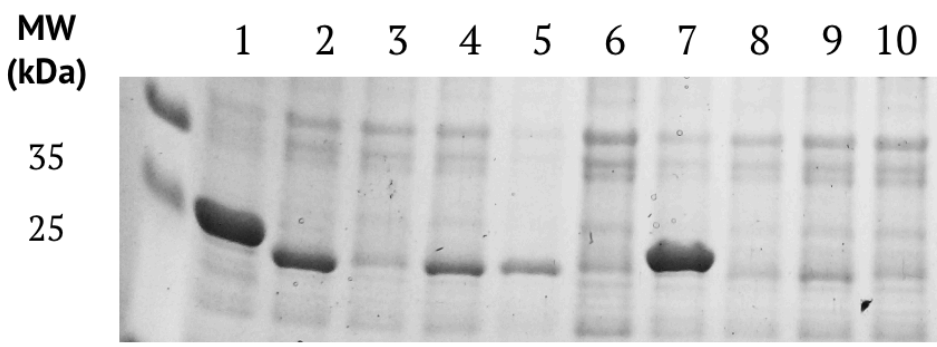

+ IPTG

$\begin{array}{llllllllllll}\text { MW } & 11 & 12 & 13 & 14 & 15 & 16 & 17 & 18 & 19 & 20 & 21\end{array}$

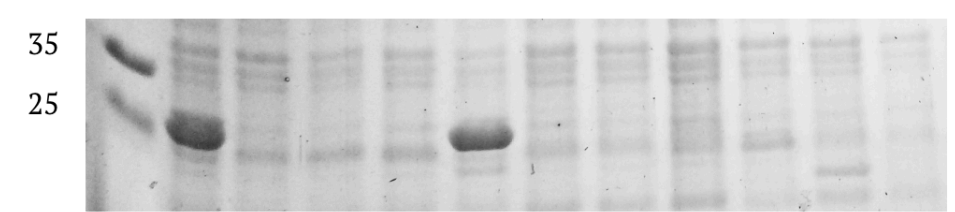

Figure 6-11 SDS-PAGE gel showing relative protein expression levels of His ${ }_{6}$-tagged nitroreductases and nitroreductase hits from multi-genomic library in 7NT. Nitroreductase-expressing cultures were grown with (+) or without (-) induction by $50 \mu M$ IPTG for 4 hours prior to collection of whole-cell lysate. Whole-cell lysate samples were normalised for cell density $\left(\mathrm{OD}_{600}\right)$ and $10 \mu \mathrm{L}$ of sample loaded per lane. 
1. His $_{6}-N f_{s} A$ Ec $(M W 29.0 \mathrm{kDa})$

2. NfSA_Ec, forward, starts $106 \mathrm{bp}$ downstream from NcoI (MW $26.8 \mathrm{kDa})$

3. NfSA_Ec, forward, starts $182 \mathrm{bp}$ downstream from NcoI (MW $26.8 \mathrm{kDa})$

4. NfSA_Ec, forward, starts $630 \mathrm{bp}$ downstream from NcoI (MW $26.8 \mathrm{kDa})$

5. NfSA_Ec, forward, starts $782 \mathrm{bp}$ downstream from NcoI (MW $26.8 \mathrm{kDa})$

6. $\quad N f S A \_E c$, reverse $(M W 26.8 \mathrm{kDa})$

7. $\mathrm{His}_{6}-\mathrm{Nfs} B \mathrm{Ec}(\mathrm{MW} 26.1 \mathrm{kDa})$

8. Nfs__Ec, forward, starts 11 bp downstream from NcoI (MW $23.9 \mathrm{kDa})$

9. Nfs $B_{-} E c$, forward, starts 234 bp downstream from NcoI (MW $23.9 \mathrm{kDa}$ )

10. $N f_{s} B$ Ec , reverse $(M W 23.9 \mathrm{kDa})$

11. $\mathrm{His}_{6}-\mathrm{NfS} \mathrm{f}_{-} \mathrm{Ck}(29.0 \mathrm{kDa})$

12. NfsA_Ck, forward, start codon encoded for by NcoI (26.9 kDa)

13. NfsA_Ck, forward, starts $259 \mathrm{bp}$ downstream from NcoI (26.9 kDa)

14. NfSA_Ck, forward, starts $511 \mathrm{bp}$ downstream from NcoI (26.9 $\mathrm{kDa})$

15. $\mathrm{His}_{6}-\mathrm{Nfs} \mathrm{B}_{-} \mathrm{Ck}(26.1 \mathrm{kDa})$

16. $N f_{s} B_{-} C k$, forward, starts 261 bp downstream from NcoI (23.9 kDa)

17. $N f_{s} B_{-} C k$, reverse $(23.9 \mathrm{kDa})$

18. $\mathrm{His}_{6}-\mathrm{Nfs} \mathrm{B}_{-} \mathrm{Pp}(26.4 \mathrm{kDa})$

19. Nfs__Pp, forward, start codon encoded for by NcoI $(24.2 \mathrm{kDa})$

20. YodC (B. subtilis), forward, starts $99 \mathrm{bp}$ downstream from NcoI (22.3 kDa)

21. YodC (B. subtilis), reverse (22.3 $\mathrm{kDa})$ 


\subsection{Discussion}

This chapter describes the utilization of a novel cloning strategy for the proof-of-principle creation of a multi-genomic library, and subsequent discovery of nitroreductases from this library using nitroaromatic positive selection and screening compounds. We demonstrated the successful selection of a variety of nitroreductase enzymes, the majority of which came from E. coli W3110. Despite apparent high levels of contaminating E. coli DNA resulting in a dominating percentage of $n f s A_{-} E c$ and $n f s B_{-} E c$ genes being recovered in this proof-of-principle study, the successful recovery of the $n f s A_{-} C k$ and $n f s B_{-} P p$ genes from this library indicated that FatI partial digestion is potentially a useful strategy for recovery of nitroreductase genes from a metagenomic library. It remains to be seen if the abundance of $E$. coli enzymes found in this library is due to simply to an over-representation of the W3110 genomic DNA, and we have yet to determine if nitroreductases in other $E$. coli genomes contained in soil DNA would be as highly represented in future metagenomic libraries. Deliberate selection of soil from areas where remnants of gastrointestinal $E$. coli species are less likely to occur should allow for the avoidance of this issue. Of the 10 nitroreductase enzymes likely present in the library that contained a FatI restriction site immediately preceding the start codon, only two of these (NfsA_Ck and NfsB_Pp) were found following screening of the library with niclosamide and 4-nitroimidazole or 2-methyl-4-nitroimidazole. This may have been partly due to the overwhelming number of multi-genomic fragments containing NfsA_Ec, which made up $81 \%$ of counter-screened variants. No nitroreductase enzymes from $B$. thailandensis were discovered in the variants sequenced in this chapter, although it is possible that none of the annotated proteins exhibit activity with niclosamide or 4-nitroimidazole or 2-methyl4-nitroimidazole. Due to the success of using pET28a+ as a cloning vector for this multi-genomic library, we postulate that it would be an ideal vector for future cloning of soil metagenomic libraries, albeit with some minor modifications. To reduce the risk of contamination with other nitroreductase sources in the Ackerley lab, the kanamycin resistance 
cassette in pET28a + could be replaced with an alternative antibiotic resistance cassette. For example, Manna et al. (2013) demonstrated the successful disruption of $a m p^{R}$ and $k a n^{R}$ genes in a variety of vectors through the insertion of a tetracycline resistance cassette $\left(\right.$ tet $\left.^{R}\right)$, which had no effect on plasmid copy numbers, and the same strategy could be utilised for the insertion of $t e t^{R}$ in place of $\mathrm{kan}^{R}$ in pET28a+. Furthermore, the substitution of the modified pET28a + MCS (as described in Section 6.3.2.1) would allow for $\mathrm{His}_{6}$-tag expression of FatI-cloned nitroreductases, which may also increase the overall expression of these enzymes and allow for a higher proportion of nitroreductase genes (relative to tolC-like genes) to be recovered from the library. However a lower proportion of survival of 7NT cells over-expressing some nitroreductases (e.g. NfsB_Pp) in the pET28a+ vector on LB agar plates containing $0.5 \mu \mathrm{M}$ niclosamide was noted, relative to the ability of their pUCX-cloned counterparts to detoxify niclosamide in liquid media. This could reflect the lower copy number of plasmids with a pBR322 origin of replication (e.g. pET28a+) relative to those with a mutated pUC origin (e.g. pUCX; Lin-Chao et al., 1992), and suggests that the use of a plasmid containing the latter may allow for the recovery of more nitroreductase genes. Finally, due to the high proportion of nitroreductases discovered that were not cloned in using the FatI strategy, we have yet to determine if metagenomic library creation using this enzyme would allow for the discovery of more nitroreductase genes than cleavage of metagenomic DNA using a less specific endonuclease, such as DNaseI. Direct comparison of these two strategies would allow for the determination of whether the former strategy actually improves our ability to recover nitroreductases from the library, or whether it is more likely to miss nitroreductase genes that contain a large number of internal FatI restriction sites. The FatI cloning strategy may also be useful for discovery of any kind of enzyme from a metagenomic library where high-level expression is required to facilitate effective screening.

The counter-screening of niclosamide-detoxifying colonies with 4nitroimidazole and 2-methyl-4-nitroimidazole from LB agar plates was also 
successful; however, it was likely one of the factors contributing to the increased recovery of $n f S A \_E c$ genes from this multi-genomic library, as it appeared to demonstrate very strong activation of both nitro-masked chromophores. The selection of colonies that can activate these compounds on LB agar plates from metagenomic libraries may also be more likely to recover nitroreductase variants from the NfsA family, as their colonies are likely to exhibit the strongest colour change. Thus for future screening of metagenomic libraries, counter-screening of niclosamide-detoxifying variants in liquid assays with nitro-masked chromophores as well as other nitroaromatic compounds (e.g. metronidazole or nitrofurazone) may allow for more successful recovery of enzymes from alternative nitroreductase families. 


\section{Chapter 7. Key findings, conclusions and future directions}

\subsection{Research motivation}

The research described in this thesis involved the engineering of nitroreductase candidates, primarily for their potential use in human GDEPT clinical trials. Our collaborators in this work have sought to improve on the original NfsB/CB1954 enzyme-prodrug GDEPT pairing through the creation of next-generation prodrugs with improved pharmacological attributes as well as the engineering of specialised tumour-targeting $C$. sporogenes vectors for gene delivery to tumour tissues. Other (present and past) members of the Ackerley lab have focused on the improvement of the activity of nitroreductase candidates with these prodrugs, and more recently, with PET imaging probes. One of the key limitations in human GDEPT trials is the inability to rapidly and non-invasively confirm localisation of nitroreductase genes in the patient prior to systemic prodrug administration, and our lab has thus sought to use PET imaging probes that can be activated by nitroreductase enzymes to cell-entrapped forms to allow for PET scans to determine gene location. The initial research conducted in the Ackerley lab in this field focused on the adaptation of 2-nitroimidazole PET imaging probes, which had originally been developed for imaging of hypoxic tissues, for nitroreductase activation (e.g., Copp et al., 2017). However, the comparison of PET imaging of NfsA_Ec nitroreductaseexpressing tumours in mice versus untransfected tumours revealed relatively high background activation of the probe due to hypoxia, suggesting that reduced sensitivity would be an issue even with evolved nitroreductase enzymes. Therefore my efforts were focused on bespoke 5-nitroimidazole PET imaging probes designed by our collaborators exclusively for nitroreductase activation, as they possess a sufficiently low one-electron transfer potential to avoid being activated by human enzymes under hypoxic conditions. 


\subsection{Summary of key findings}

\subsubsection{Discovery of wild-type nitroreductases that exhibited activity with S33}

Through the screening of a 58 nitroreductase library, which comprises wildtype nitroreductase candidates from a range of enzyme families and bacterial species, I discovered a substantial number from the NfsA and NfsB families that demonstrated the ability to activate S33 in E. coli. From the latter family, only a subset of nitroreductases that shared less than $45 \%$ identity with E. coli NfsB exhibited activity with this compound. Further analysis revealed that the NfsA enzymes YcnD_Bs and NfsA_Ec, and the NfsB enzyme YfkO_Bs were amongst the most active S33 nitroreductases in our collection, and these were selected for further study. For the analysis of kinetic parameters of these enzymes with S33, their high background NADPH oxidase activity relative to their activity with S33 (i.e., enzymatic transfer of electrons donated by NADPH directly to oxygen molecules present in the solution instead of S33) meant that Michaelis-Menten kinetic parameters for reduction of S33 were unable to be directly measured. As an alternative method, we settled on the use of a glucose oxidase-catalase oxygen scavenging system to remove oxygen from the solution, allowing for rates of reduction to be measured with a higher degree of accuracy (albeit still with large errors). The estimated $k_{\text {cat }} / K_{M}$ for the YcnD_Bs, NfsA_Ec, and YfkO_Bs nitroreductases with S33 were all below $10^{3} \mathrm{~s}^{-1} \cdot \mathrm{M}^{-1}$, which is low relative to the discernible levels activity they exhibited in vivo and may indicate inaccuracies in our calculation of the extinction coefficient for S33. Nevertheless, throughout this work the general methodology was primarily used to compare levels of in vitro activity between different wild type enzymes and evolved variants thereof, and an inaccurate extinction coefficient would not interfere with these relative comparisons. 


\subsubsection{Screening site-targeted libraries for variants exhibiting improved activity with S33}

For the initial evaluation of evolved nitroreductase variants that could activate S33, I screened previously created site-targeted libraries of the NfsA enzymes YcnD_Bs and NfsA_Ec, which had been designed by previous members in the Ackerley lab to improve the activity of these enzymes with the potential GDEPT prodrug PR-104A (Copp et al., 2014; Prosser, 2011). Screening of these libraries with S33 was conducted using the lacZ SOS assay in the E. coli SOS-R2 strain, which reports on the level of endogenous E. coli SOS response that is activated in response to sub-lethal DNA damage in the cell. The SOS-R2 strain contains a chromosomally integrated lacZ gene fused downstream of an sfiA SOS gene promoter, and has previously been validated for the quantification of nitroreductase activity with sub-lethal concentrations of DNA-damaging prodrugs (Prosser et al., 2013) and S33 (Williams, 2013). The variant most active with S33 that was recovered from the YcnD_Bs SSM library contained an accidental amino acid substitution, I163V, as well as the site-targeted substitution Y227V, and demonstrated a ca. 3-fold improvement in $k_{\text {cat }} / K_{M}$ over wild-type YcnD_Bs. From the NfsA_Ec SDM library, the lead variant only had one amino acid substitution, R225A in the active site, and exhibited a much higher 14 -fold improvement in $k_{c a t} / K_{M}$ over wild-type NfsA_Ec.

\subsubsection{Creation and screening of random mutagenesis libraries for improved S33 activation}

Our lab was also interested in the evolution of an NfsB enzyme e.g. YfkO_Bs for improved S33 activation for two key reasons: we have observed in the past that NfsB enzymes tend to exhibit higher $k_{c a t}$ and $K_{M}$ kinetic parameters with given substrates, and we sought to determine whether this might translate to superior activity with S33 in vivo; secondly, our collaborators have found NfsB family nitroreductases to generally be more tolerated and functional in C. sporogenes than NfsA family enzymes, albeit based on fairly 
small sample sizes. Owing to the lack of a solved crystal structure for YfkO_Bs, we utilised a random mutagenesis approach for evolution, using a low-fidelity DNA polymerase to introduce random base substitutions into the gene during PCR amplification. Simultaneously, we created a random mutagenesis library for YcnD_Bs, in an attempt to generate variants that were more active with S33 than those found from the site-targeted library. Due to the substantially larger library sizes that can theoretically be created for error-prone libraries, and the higher probability of the variants in these libraries to simply be non-functional, we utilised the positive nitroaromatic selection compound, niclosamide, to first remove generally inactive nitroreductases from the pool. For screening of the remaining variants for their activity with S33, we used the GFP SOS assay, which utilises E. coli strains bearing the $s f i A$ promoter transcriptionally fused to a plasmid-based $g f p$ gene and has been shown to permit ultra-high-throughput screening (Copp et al., 2014, 2017).

The lead four variants discovered from each of $y f k O$ and $y c n D$ libraries following the first rounds of random mutagenesis and cell sorting were used as a mixed template for generation of enzymes for the second found. Two rounds of directed evolution conducted on $\mathrm{YfkO}_{-} \mathrm{Bs}$ generated a variant with two amino acid substitutions (F82L, M110I) that exhibited a 6-fold improvement in $k_{c a t} / K_{M}$ with S33 over wild-type YfkO_Bs. This variant also demonstrated an improved ability to activate the next-generation prodrug SN36506, and a proprietary next-generation single-fluorine analogue of S33, SN37819. This error-prone evolution approach was less successful in improving the activity of $\mathrm{YcnD}$, with the top variants recovered having almost no appreciable improvements in activity with S33, due to their comparison during screening to a wild-type YcnD_Bs control that expressed at far low levels (which was not discovered until after two rounds of directed evolution). 


\subsubsection{Use of nitroreductase positive selection compounds for the discovery of improved S33-activating variants}

As an extension of the use of low levels of niclosamide to enrich

nitroreductase libraries for active enzymes, we investigated whether higher concentrations of niclosamide or another nitroaromatic positive selection compound, chloramphenicol, could be utilised as sole selection agents for nitroreductases that exhibited improved activity with S33. For this research we utilised a site-targeted NfsA_Ec library that had been synthesised for the Ackerley lab, with degenerate codons at seven key active site residues, allowing for up to 20 amino acid substitutions at each site for a total of 96 million possible unique variants. The use of concentrations of niclosamide or chloramphenicol, above that which wild-type NfsA_Ec could detoxify, selected for under $1 \%$ of variants within the $7 \mathrm{SM}$ library. To determine the effects of these selection pressures on activities with other substrates, a small subset of variants recovered post-selection with either niclosamide or chloramphenicol were counter-screened against a panel of six structurally diverse nitroaromatic compounds. Both niclosamide and chloramphenicol exhibited the capacity to enrich the pool of NfsA_Ec variants for those able to sensitise $E$. coli to lethal concentrations of all six compounds.

Furthermore, a subset of selected variants exhibited higher levels of activity than wild-type NfsA_Ec, demonstrating that these positive selection compounds could be utilised as generic agents for selection of improved nitroreductases. Within the subsets of variants screened we discovered two NfsA_Ec variants, each containing five different amino acid substitutions, that upon preliminary kinetic analysis appeared to exhibit over 4-fold improvements in $k_{c a t} / K_{M}$ with S33 relative to wild-type NfsA_Ec.

\subsubsection{Utilising nitroaromatic selection and screening compounds for discovery of new nitroreductase enzymes}

Finally, we sought to discover nitroreductases from nature that exhibited higher activity with S33 than any of the wild-type or evolved nitroreductase 
variants evaluated thus far. Due to time constraints we were unable to successfully create a suitable soil metagenome library; however a small proof-of-principle library was created from bacterial genomic DNA using a novel cloning strategy that we predicted would allow for improved expression of nitroreductase genes from metagenomic fragments in E. coli. From this library we demonstrated that both nitroreductase and tolC efflux pump-encoding genes could be pulled out from the library following selection on low levels of niclosamide, and counter-screening using nitromasked colourmetric compounds allowed for the successful recovery of solely nitroreductase-expressing gene fragments. We predict that this will allow for the future discovery of nitroreductase enzymes from unculturable bacterial strains, some of which may not presently have annotated homologues.

\subsection{Critical evaluations of methods used in this research}

\subsection{1 pUCX expression plasmid}

The use of pUCX as a nitroreductase expression plasmid over the course of this research was initially confounding due to the existence of two forms of the vector, one of which contained mutations that led to a much lower expression of nitroreductase enzymes in E. coli. Since this discovery, we have switched to the sole use of the former vector where possible; but in certain instances, such as the 7SM library which was already cloned into low-expressing pUCX, the re-cloning of a wild-type control into lowexpressing pUCX for comparison was required. However, in the case of the former plasmid, the increased levels of nitroreductase expression from highexpressing pUCX might not be ideal in all circumstances; e.g. wild-type YcnD_Bs, following induction with either 25 or $50 \mu \mathrm{M}$ IPTG, sensitised E. coli to similar concentrations of metronidazole. This suggests that the overexpressed enzyme may in fact be depleting the intracellular NADPH pools in cells, and may not allow for accurate determination of the activity of improved variants with metronidazole or other nitroaromatic substrates in 
vivo. To determine if this is indeed the case during $\mathrm{IC}_{50}$ assays, enzyme expression could be induced with several different concentrations of IPTG, to determine the highest concentration of IPTG at which a variant is still capable of sensitising $E$. coli to the same fold-lower concentrations of a compound (relative to a wild-type control). In many cases it may be necessary to use lower concentrations of IPTG for the induction of overexpressed variant nitroreductases, in particular if the wild-type or other parent enzyme utilised for comparison already exhibits high levels of activity with the compound. We showed that the $\mathrm{IC}_{50}$ values of 7NT cells over-expressing YcnD_Bs with metronidazole could be determined without any IPTG induction whatsoever, suggesting that there was still substantial leaky expression of this nitroreductase to sensitise E. coli to the prodrug. Alternatively, the inclusion of higher concentrations of glucose in the growth and challenge media (our lab uses $0.4 \%$ glucose in overnight cultures and $0.2 \%$ glucose in challenge media) could be utilised for increased repression of the lac promoter.

\subsubsection{GFP SOS assay and FACS}

The research conducted in this thesis revealed limitations on the use of the gfpmut3b gene as a plasmid-based reporter for prodrug or PET probeinduced DNA damage in E. coli. It was shown that the background levels of fluorescence could confound the fluorescence induced following nitroreductase activation of SOS-inducing compounds, which potentially limits the efficacy of selecting for improved nitroreductase candidates using FACS. Furthermore, we have shown that the presence of background GFP affects the sensitivity of $E$. coli cells to lethal concentrations of these compounds. A potential way to fix the former issue could involve the inclusion of a lexA regulator gene on the GFP plasmid to allow for more basal repression of the $s f i A$ promoter. Alternatively, the use of photoswitchable fluorescent proteins, which switch colour following activation by a particular wavelength of light (Zhou and Lin, 2013), might 
allow for the 'resetting' of the basal fluorescence prior to challenge with DNA-damaging compounds; although some of these proteins require activation using UV light (Wiedenmann et al., 2004) which might in itself activate the SOS response (Janion, 2008) and further confound the results. Alternatively, there are a wide panel of alternative blue, cyan, yellow, and red fluorescent proteins that have been produced by many research groups (Day and Davidson, 2009; Piatkevich and Verkhusha, 2011) that may not have a cytotoxic effect in E. coli, with the further advantage that excitation of fluorescent proteins at longer wavelengths than those required for the detection of GFP might diminish the background signal due to E. coli autofluorescence.

\subsubsection{Enzyme evolution and protein fitness}

The majority of directed evolution research conducted in the Ackerley lab has focused on the generation of variants having slight amino acid differences to parent enzymes, followed by screening for variants exhibiting improved activity with a particular substrate, and the use of these variants as templates for further rounds of mutagenesis. However, substantial amounts of research suggest that this traditional approach, whereby evolved enzyme variants are 'climbing a peak' towards a local fitness maximum i.e. the most active enzyme achievable with the substrate in question, may not allow for the most active variant within the entire landscape to be revealed (Poelwijk et al., 2007; Weinreich et al., 2006). More specifically, it tends to ignore the effects of individual amino acid substitutions that on their own may have little or a negative impact on enzyme activity (i.e. dipping into a fitness 'valley'); but that have the potential to exhibit beneficial epistatic interactions with other altered residues (Bornscheuer et al., 2012; Dalby, 2011). For example, Steinberg and Ostermeier utilised four different evolution strategies to evolve the antibiotic resistance gene TEM-15 $\beta$ lactamase using comprehensive saturation mutagenesis - strict positive selection, neutral selection, negative selection, and oscillating selection (the 
latter three strategies employing three rounds of that particular selection, followed by a further five rounds of positive selection; Steinberg and Ostermeier, 2016). Variants were discovered following the negative selection strategy that exhibited upwards of four-fold improvements in activity over the best variants resulting from eight rounds of strictly positive selection (Steinberg and Ostermeier, 2016).

Other researchers have also shown that neutral drift selection, where enzymes are subjected to mutagenesis and then selected for the retention of generic enzyme activity, can generate pools of enzymes that are highly evolvable when subjected to a new selection pressure (Bershtein et al., 2008). Based on our demonstration that niclosamide and chloramphenicol can both be used to apply a purifying selection that eliminates inactive enzyme variants from a pool while still permitting a very large number of simultaneous mutations to accrue, we propose that these compounds could be used in a similar fashion to enable "guided drift" - that is, enzyme variants that have traversed broad distances across a fitness landscape, but still retain some form of generic nitroreductase activity.

Finally, another potential strategy for directed evolution that has yet to be undertaken by the Ackerley lab involves the initial generation of enzymes exhibiting improved stability (e.g. thermostability) over wild-type parent enzymes. Other research groups have shown that enhancing the overall stability of enzymes in early rounds of evolution can promote their capacity to tolerate more destabilizing active site mutations involved in the specific alteration of enzymatic activity with a particular substrate (Bloom et al., 2006). In this regard, there has been renewed interest in the field of ancestral enzyme reconstruction, where the ancestral sequence is conferred from alignments and phylogenetic analysis of modern derived enzymes (Gumulya and Gillam, 2017). Ancestral enzymes are generally regarded as being useful both for their frequent ability to activate a wider range of substrates than more specialised modern enzymes, and for usually exhibiting much higher thermostability and broader $\mathrm{pH}$ tolerance (Alcalde, 
2017). The construction of ancestral nitroreductase sequences may therefore allow for the subsequent generation of variant libraries containing a larger proportion of stable enzymes that may also exhibit activity with many different nitroaromatic substrates.

\subsection{Future directions}

The research described in this thesis achieved varying levels of success of the engineering of nitroreductase enzymes for improved activation of S33. We have generated NfsA_Ec and YfkO_Bs variants have that exhibit improved activity both in E. coli and in vitro, and there is great potential for further directed evolution (e.g., using epPCR) of lead NfsA_Ec or YfkO_Bs variants to further improve their activity with S33. However we have not yet shown the ability of these variants to stably express in human cell lines, or demonstrated their improved activation of S33 over wild-type parent nitroreductases in tumour cells. Previous research conducted in the Ackerley lab has shown that we are not yet capable of predicting the ability of individual nitroreductase variants to be tolerated in human cell lines (Copp et al., 2017; Prosser et al., 2013; Williams, 2013), and furthermore we have noted that variants exhibiting strong improvements in the ability to sensitise $E$. coli cells to lethal concentrations of prodrugs do not always demonstrate the same capacity to do so in human cells. Confirmation of the improved ability of our evolved nitroreductase variants to activate S33 in human cell lines can be rapidly conducted following incubation with a fluorescent anti-S33 antibody and visualization of the shift in fluorescence of cells in a flow cytometer. In the future, this will be confirmed for the most promising variants generated in this study, prior to sending stablytransfected tumour cells lines (such as the HCT-116 colon carcinoma line that we commonly work with in the Ackerley lab, e.g. Copp et al., 2017; Prosser et al., 2013) to our Radiation Oncology collaborators at the University of Maastricht for evaluation in mouse micro-PET models. If these variants demonstrate improved activation of S33 in these micro-PET 
models, the ability to active SN36506 as well as capable integration into $C$. sporogenes spores, they could potentially be utilised in human clinical trials as tumour-targeted enzymes whose localisation can be confirmed by PET imaging prior to activation of a GDEPT prodrug for specific ablation of cancerous tissues. 


\section{References}

Ackerley, D.F., and Copp, J.N. (2016). Salicylamide antibiotics. PCT Patent Application WO2016080846.

Ackerley, D.F., Gonzalez, C.F., Keyhan, M., Blake, R., and Matin, A. (2004). Mechanism of chromate reduction by the Escherichia coli protein, NfsA, and the role of different chromate reductases in minimizing oxidative stress during chromate reduction. Environ. Microbiol. 6, 851-860.

Aharoni, A., Gaidukov, L., Khersonsky, O., McQ Gould, S., Roodveldt, C., and Tawfik, D.S. (2005). The "evolvability" of promiscuous protein functions. Nat. Genet. 37, 73-76.

Alcalde, M. (2017). When directed evolution met ancestral enzyme resurrection. Microb. Biotechnol. 10, 22-24.

Al-Hadiya, B.M.H. (2005). Niclosamide: comprehensive profile. Profiles Drug Subst. Excip. Relat. Methodol. 32, 67-96.

Alksne, L.E., Burgio, P., Hu, W., Feld, B., Singh, M.P., Tuckman, M., Petersen, P.J., Labthavikul, P., McGlynn, M., Barbieri, L., McDonald, L., Bradford, P., Dushin, R.G., Rothstein, D., and Projan, S.J. (2000). Identification and analysis of bacterial protein secretion inhibitors utilizing a SecA-LacZ reporter fusion system. Antimicrob. Agents Chemother. 44, 1418-1427.

Andersen, J.B., Sternberg, C., Poulsen, L.K., Bjorn, S.P., Givskov, M., and Molin, S. (1998). New unstable variants of green fluorescent protein for studies of transient gene expression in bacteria. Appl. Environ. Microbiol. 64, 2240-2246.

Anderson, R.F., Smaill, J.B., Patterson, A.V., Ashoorzadeh, A., Ackerley, D.F., Copp, J.N., Mowday, A.M., Williams, E.M., Guise, C.P., Koch, C., Kachur, A., and Dolbier Jr, W.R. (2014). Compounds and methods for selective imaging and/or ablation. WO2014007650 A1.

Andtbacka, R.H.I., Kaufman, H.L., Collichio, F., Amatruda, T., Senzer, N., Chesney, J., Delman, K.A., Spitler, L.E., Puzanov, I., Agarwala, S.S., Milhem, M., Cranmer, L., Curti, B., Lewis, K., Ross, M., Guthrie, T., Linette, G.P., Daniels, G.A., Harrington, K., Middleton, M.R., Miller, W.H., Zager, J.S., Ye, Y., Yao, B., Li, A., Doleman, S., VanderWalde, A., Gansert, J., and Coffin, R.S. (2015). Talimogene laherparepvec improves durable response rate in patients with advanced melanoma. J. Clin. Oncol. 33, 2780-2788.

Anlezark, G.M., Melton, R.G., Sherwood, R.F., Coles, B., Friedlos, F., and Knox, R.J. (1992). The bioactivation of 5-(aziridin-1-yl)-2,4dinitrobenzamide (CB1954)--I. Purification and properties of a nitroreductase enzyme from Escherichia coli--a potential enzyme for 
antibody-directed enzyme prodrug therapy (ADEPT). Biochem. Pharmacol. $44,2289-2295$.

Anlezark, G.M., Vaughan, T., Fashola-Stone, E., Michael, N.P., Murdoch, H., Sims, M.A., Stubbs, S., Wigley, S., and Minton, N.P. (2002). Bacillus amyloliquefaciens orthologue of Bacillus subtilis ywrO encodes a nitroreductase enzyme which activates the prodrug CB 1954. Microbiology 148, 297-306.

Baban, C.K., Cronin, M., O’Hanlon, D., O’Sullivan, G.C., and Tangney, M. (2010). Bacteria as vectors for gene therapy of cancer. Bioeng. Bugs 1, 385394.

Bai, J., Yang, J., Zhou, Y., and Yang, Q. (2015a). Structural basis of Escherichia coli nitroreductase NfsB triple mutants engineered for improved activity and regioselectivity toward the prodrug CB1954. Process Biochem. 50, 1760-1766.

Bai, J., Yang, J., Liu, P., and Yang, Q. (2015b). Transformation pathway of 2,4,6-trinitrotoluene by Escherichia coli nitroreductases and improvement of activity using structure-based mutagenesis. Process Biochem. 50, 705-711.

Barak, Y., Thorne, S.H., Ackerley, D.F., Lynch, S.V., Contag, C.H., and Matin, A. (2006a). New enzyme for reductive cancer chemotherapy, YieF, and its improvement by directed evolution. Mol. Cancer Ther. 5, 97-103.

Barak, Y., Ackerley, D.F., Dodge, C.J., Banwari, L., Alex, C., Francis, A.J., and Matin, A. (2006b). Analysis of novel soluble chromate and uranyl reductases and generation of an improved enzyme by directed evolution. Appl. Environ. Microbiol. 72, 7074-7082.

Barker, H.E., Paget, J.T.E., Khan, A.A., and Harrington, K.J. (2015). The tumour microenvironment after radiotherapy: mechanisms of resistance and recurrence. Nat. Rev. Cancer 15, 409-425.

Belmar, C., So, P.W., Vassaux, G., Moleiro-Sanemeterio, V., and MartínDuque, P. (2007). Non-invasive genetic imaging for molecular and cell therapies of cancer. Clin. Transl. Oncol. 9, 703-714.

Beristain-Castillo, E., Martínez-Vázquez, M., Camacho-Carranza, R., and Espinosa-Aguirre, J.J. (2013). CYP1A1 and Cnr nitroreductase bioactivated niclosamide in vitro. Mutagenesis 28, 645-651.

Bernier, J., Hall, E.J., and Giaccia, A. (2004). Radiation oncology: a century of achievements. Nat. Rev. Cancer 4, 737-747.

Bershtein, S., Goldin, K., and Tawfik, D.S. (2008). Intense neutral drifts yield robust and evolvable consensus proteins. J. Mol. Biol. 379, 1029-1044. 
Bhaumik, S. (2011). Advances in imaging gene-directed enzyme prodrug therapy. Curr. Pharm. Biotechnol. 12, 497-507.

Bhaumik, S., Sekar, T.V., Depuy, J., Klimash, J., and Paulmurugan, R. (2012). Noninvasive optical imaging of nitroreductase gene-directed enzyme prodrug therapy system in living animals. Gene Ther. 19, 295-302.

Bloom, J.D., Labthavikul, S.T., Otey, C.R., and Arnold, F.H. (2006). Protein stability promotes evolvability. Proc. Natl. Acad. Sci. U.S.A. 103, 5869-5874.

Boland, M.P., Knox, R.J., and Roberts, J.J. (1991). The differences in kinetics of rat and human DT diaphorase result in a differential sensitivity of derived cell lines to CB 1954 (5-(aziridin-1-yl)-2,4-dinitrobenzamide). Biochem. Pharmacol. 41, 867-875.

Bornscheuer, U.T., Huisman, G.W., Kazlauskas, R.J., Lutz, S., Moore, J.C., and Robins, K. (2012). Engineering the third wave of biocatalysis. Nature 485, 185-194.

Brader, P., Serganova, I., and Blasberg, R.G. (2013). Noninvasive molecular imaging using reporter genes. J. Nucl. Med. 54, 167-172.

Bridgewater, J.A., Knox, R.J., Pitts, J.D., Collins, M.K., and Springer, C.J. (1997). The bystander effect of the nitroreductase/CB1954 enzyme/prodrug system is due to a cell-permeable metabolite. Hum. Gene Ther. 8, 709-717.

Brown, J.M., and Wilson, W.R. (2004). Exploiting tumour hypoxia in cancer treatment. Nat. Rev. Cancer 4, 437-447.

Bryant, C., and DeLuca, M. (1991). Purification and characterization of an oxygen-insensitive NAD(P)H nitroreductase from Enterobacter cloacae. J. Biol. Chem. 266, 4119-4125.

Bryant, D.W., McCalla, D.R., Leeksma, M., and Laneuville, P. (1981). Type I nitroreductases of Escherichia coli. Can. J. Microbiol. 27, 81-86.

Caballero, A., Lázaro, J.J., Ramos, J.L., and Esteve-Núñez, A. (2005). PnrA, a new nitroreductase-family enzyme in the TNT-degrading strain Pseudomonas putida JLR11. Environ. Microbiol. 7, 1211-1219.

Cao, J., Campbell, J., Liu, L., Mason, R.P., and Lippert, A.R. (2016). In vivo chemiluminescent imaging agents for nitroreductase and tissue oxygenation. Anal. Chem. 88, 4995-5002.

Chabner, B.A., and Roberts, T.G. (2005). Chemotherapy and the war on cancer. Nat. Rev. Cancer 5, 65-72.

Chen, S., Knox, R., Wu, K., Deng, P.S., Zhou, D., Bianchet, M.A., and Amzel, L.M. (1997). Molecular basis of the catalytic differences among DTdiaphorase of human, rat, and mouse. J. Biol. Chem. 272, 1437-1439. 
Chiocca, E.A. (2002). Oncolytic viruses. Nat. Rev. Cancer 2, 938-950.

Chiocca, E.A., and Rabkin, S.D. (2014). Oncolytic viruses and their application to cancer immunotherapy. Cancer Immunol. Res. 2, 295-300.

Choi, J.W., Lee, J., Nishi, K., Kim, Y.S., Jung, C.H., and Kim, J.S. (2008). Crystal structure of a minimal nitroreductase, ydjA, from Escherichia coli K12 with and without FMN cofactor. J. Mol. Biol. 377, 258-267.

Chung-Faye, G., Palmer, D., Anderson, D., Clark, J., Downes, M., Baddeley, J., Hussain, S., Murray, P.I., Searle, P., Seymour, L., Harris, P.A., Ferry, D., and Kerr, D.J. (2001). Virus-directed, enzyme prodrug therapy with nitroimidazole reductase: a Phase I and pharmacokinetic study of its prodrug, CB1954. Clin. Cancer Res. 7, 2662-2668.

Clairmont, C., Lee, K.C., Pike, J., Ittensohn, M., Low, K.B., Pawelek, J., Bermudes, D., Brecher, S.M., Margitich, D., Turnier, J., Li, Z., Luo, X., King, I., and Zheng, L.M. (2000). Biodistribution and genetic stability of the novel antitumor agent VNP20009, a genetically modified strain of Salmonella typhimurium. J. Infect. Dis. 181, 1996-2002.

Cloughesy, T.F., Landolfi, J., Hogan, D.J., Bloomfield, S., Carter, B., Chen, C.C., Elder, J.B., Kalkanis, S.N., Kesari, S., Lai, A., Lee, I.Y., Liau, L.M., Mikkelsen, T., Nghiemphu, P.L., Piccioni, D., Walbert, T., Chu, A., Das, A., Diago, O.R., Gammon, D., Gruber, H.E., Hanna, M., Jolly, D.J., Kasahara, N., McCarthy, D., Mitchell, L., Ostertag, D., Robbins, J.M., Rodriguez-Aguirre, M., and Vogelbaum, M.A. (2016). Phase 1 trial of vocimagene amiretrorepvec and 5-fluorocytosine for recurrent high-grade glioma. Sci. Transl. Med. 8, 341ra75.

Cobb, L.M., Connors, T.A., Elson, L.A., Khan, A.H., Mitchley, B.C., Ross, W.C., and Whisson, M.E. (1969). 2,4-dinitro-5-ethyleneiminobenzamide (CB 1954): a potent and selective inhibitor of the growth of the Walker carcinoma 256. Biochem. Pharmacol. 18, 1519-1527.

Condon, S. (2013). The characterisation and application of bacterial nitroreductase enzymes. MSc Thesis. School of Biological Sciences, Victoria University of Wellington, Wellington.

Copp, J.N., Williams, E.M., Rich, M.H., Patterson, A.V., Smaill, J.B., and Ackerley, D.F. (2014). Toward a high-throughput screening platform for directed evolution of enzymes that activate genotoxic prodrugs. Protein Eng. Des. Sel. 27, 399-403.

Copp, J.N., Mowday, A.M., Williams, E.M., Guise, C.P., Ashoorzadeh, A., Sharrock, A.V., Flanagan, J.U., Smaill, J.B., Patterson, A.V., and Ackerley, D.F. (2017). Engineering a multifunctional nitroreductase for improved activation of prodrugs and PET probes for cancer gene therapy. Cell Chem. Biol. 24, 391-403. 
Cormack, B.P., Valdivia, R.H., and Falkow, S. (1996). FACS-optimized mutants of the green fluorescent protein (GFP). Gene 173, 33-38.

Craig, J.W., Chang, F.Y., Kim, J.H., Obiajulu, S.C., and Brady, S.F. (2010). Expanding small-molecule functional metagenomics through parallel screening of broad-host-range cosmid environmental DNA libraries in diverse proteobacteria. Appl. Environ. Microbiol. 76, 1633-1641.

Crameri, A., Whitehorn, E.A., Tate, E., and Stemmer, W.P. (1996). Improved green fluorescent protein by molecular evolution using DNA shuffling. Nat. Biotechnol. 14, 315-319.

Cronin, M., Stanton, R.M., Francis, K.P., and Tangney, M. (2012). Bacterial vectors for imaging and cancer gene therapy: a review. Cancer Gene Ther. 19, 731-740.

Culligan, E.P., Sleator, R.D., Marchesi, J.R., and Hill, C. (2014).

Metagenomics and novel gene discovery: promise and potential for novel therapeutics. Virulence 5, 399-412.

Curado, S., Anderson, R.M., Jungblut, B., Mumm, J., Schroeter, E., and Stainier, D.Y.R. (2007). Conditional targeted cell ablation in zebrafish: a new tool for regeneration studies. Dev. Dyn. 236, 1025-1035.

Dachs, G.U., Hunt, M.A., Syddall, S., Singleton, D.C., and Patterson, A.V. (2009). Bystander or no bystander for gene directed enzyme prodrug therapy. Molecules 14, 4517-4545.

Dalby, P.A. (2011). Strategy and success for the directed evolution of enzymes. Curr. Opin. Struct. Biol. 21, 473-480.

Daniel, R. (2005). The metagenomics of soil. Nat. Rev. Microbiol. 3, 470-478.

Day, R.N., and Davidson, M.W. (2009). The fluorescent protein palette: tools for cellular imaging. Chem. Soc. Rev. 38, 2887-2921.

Denny, W.A. (2000). The role of hypoxia-activated prodrugs in cancer therapy. Lancet Oncol. 1, 25-29.

van Dillen, I.J., Mulder, N.H., Vaalburg, W., de Vries, E.F.J., and Hospers, G.A.P. (2002). Influence of the bystander effect on HSV-tk/GCV gene therapy. A review. Curr. Gene Ther. 2, 307-322.

Ditzel, J., and Schwartz, M. (1967). Worm cure without tears. The effect of niclosamide on Taeniasis saginata in man. Acta. Med. Scand. 182, 663-664.

Dolgin, E. (2015). Oncolytic viruses get a boost with first FDA-approval recommendation. Nat. Rev. Drug Discov. 14, 369-371. 
Dri, A.M., and Moreau, P.L. (1994). Control of the LexA regulon by $\mathrm{pH}$ : evidence for a reversible inactivation of the LexA repressor during the growth cycle of Escherichia coli. Mol. Microbiol. 12, 621-629.

Duarte, S., Carle, G., Faneca, H., de Lima, M.C.P., and Pierrefite-Carle, V. (2012). Suicide gene therapy in cancer: where do we stand now? Cancer Lett. $324,160-170$.

Dunbrack, R.L. (2002). Rotamer libraries in the 21st century. Curr. Opin. Struct. Biol. 12, 431-440.

Durfee, T., Nelson, R., Baldwin, S., Plunkett, G., Burland, V., Mau, B., Petrosino, J.F., Qin, X., Muzny, D.M., Ayele, M., Gibbs, R.A., Csörgo, B., Pósfai, G., Weinstock, G.M., and Blattner, F.R. (2008). The complete genome sequence of Escherichia coli DH10B: insights into the biology of a laboratory workhorse. J. Bacteriol. 190, 2597-2606.

Edwards, D.I. (1993). Nitroimidazole drugs--action and resistance mechanisms. I. Mechanisms of action. J. Antimicrob. Chemother. 31, 9-20.

Englander, S.W., Calhoun, D.B., and Englander, J.J. (1987). Biochemistry without oxygen. Anal. Biochem. 161, 300-306.

Espinosa-Aguirre, J.J., Reyes, R.E., and Cortinas de Nava, C. (1991). Mutagenic activity of 2-chloro-4-nitroaniline and 5-chlorosalicylic acid in Salmonella typhimurium: two possible metabolites of niclosamide. Mutat. Res. 264, 139-145.

Everts, B., and van der Poel, H.G. (2005). Replication-selective oncolytic viruses in the treatment of cancer. Cancer Gene Ther. 12, 141-161.

Farkona, S., Diamandis, E.P., and Blasutig, I.M. (2016). Cancer immunotherapy: the beginning of the end of cancer? BMC Med. 14, 73.

Feder, H.M., Osier, C., and Maderazo, E.G. (1981). Chloramphenicol: a review of its use in clinical practice. Rev. Infect. Dis. 3, 479-491.

Feketeová, L., Postler, J., Zavras, A., Scheier, P., Denifl, S., and O’Hair, R.A.J. (2015). Decomposition of nitroimidazole ions: experiment and theory. Phys. Chem. Chem. Phys. 17, 12598-12607.

Felgner, S., Kocijancic, D., Frahm, M., and Weiss, S. (2016). Bacteria in cancer therapy: renaissance of an old concept. Int. J. Microbio. 2016, 8451728 .

Fernández-Martínez, L.T., Borsetto, C., Gomez-Escribano, J.P., Bibb, M.J., Al-Bassam, M.M., Chandra, G., and Bibb, M.J. (2014). New insights into chloramphenicol biosynthesis in Streptomyces venezuelae ATCC 10712. Antimicrob. Agents Chemother. 58, 7441-7450. 
Forbes, N.S. (2010). Engineering the perfect (bacterial) cancer therapy. Nat. Rev. Cancer 10, 785-794.

Frische, T. (2002). Screening for soil toxicity and mutagenicity using luminescent bacteria--a case study of the explosive 2,4,6-trinitrotoluene (TNT). Ecotoxicol. Environ. Saf. 51, 133-144.

Fukuhara, H., Ino, Y., and Todo, T. (2016). Oncolytic virus therapy: A new era of cancer treatment at dawn. Cancer Sci. 107, 1373-1379.

Gabor, E.M., Alkema, W.B.L., and Janssen, D.B. (2004). Quantifying the accessibility of the metagenome by random expression cloning techniques. Environ. Microbiol. 6, 879-886.

Galanis, E., Markovic, S.N., Suman, V.J., Nuovo, G.J., Vile, R.G., Kottke, T.J., Nevala, W.K., Thompson, M.A., Lewis, J.E., Rumilla, K.M., Roulstone, V., Harrington, K., Linette, G.P., Maples, W.J., Coffey, M., Zwiebel, J., and Kendra, K. (2012). Phase II trial of intravenous administration of Reolysin( $\left.{ }^{\circledR}\right)$ (Reovirus Serotype-3-dearing Strain) in patients with metastatic melanoma. Mol. Ther. 20, 1998-2003.

Garber, K. (2006). China approves world's first oncolytic virus therapy for cancer treatment. J. Natl. Cancer Inst. 98, 298-300.

Gollamudi, R., Ghalib, M.H., Desai, K.K., Chaudhary, I., Wong, B., Einstein, M., Coffey, M., Gill, G.M., Mettinger, K., Mariadason, J.M., Mani, S., and Goel, S. (2010). Intravenous administration of Reolysin, a live replication competent RNA virus is safe in patients with advanced solid tumors. Invest. New Drugs 28, 641-649.

González-Pérez, M.M., van Dillewijn, P., Wittich, R.M., and Ramos, J.L. (2007). Escherichia coli has multiple enzymes that attack TNT and release nitrogen for growth. Environ. Microbiol. 9, 1535-1540.

Gooyit, M., and Janda, K.D. (2016). Reprofiled anthelmintics abate hypervirulent stationary-phase Clostridium difficile. Sci. Rep. 6, 33642.

Gould, S.M., and Tawfik, D.S. (2005). Directed evolution of the promiscuous esterase activity of carbonic anhydrase II. Biochemistry 44, 5444-5452.

Green, L.K., Storey, M.A., Williams, E.M., Patterson, A.V., Smaill, J.B., Copp, J.N., and Ackerley, D.F. (2013). The flavin reductase MsuE is a novel nitroreductase that can efficiently activate two promising next-generation prodrugs for gene-directed enzyme prodrug therapy. Cancers (Basel) 5, 985997.

Green, L.K., La Flamme, A.C., and Ackerley, D.F. (2014). Pseudomonas aeruginosa MdaB and WrbA are water-soluble two-electron quinone oxidoreductases with the potential to defend against oxidative stress. J. Microbiol. 52, 771-777. 
Grove, J.I., Lovering, A.L., Guise, C., Race, P.R., Wrighton, C.J., White, S.A., Hyde, E.I., and Searle, P.F. (2003). Generation of Escherichia coli nitroreductase mutants conferring improved cell sensitization to the prodrug CB1954. Cancer Res. 63, 5532-5537.

Guay, D.R. (2001). An update on the role of nitrofurans in the management of urinary tract infections. Drugs $61,353-364$.

Guise, C.P., Abbattista, M.R., Singleton, R.S., Holford, S.D., Connolly, J., Dachs, G.U., Fox, S.B., Pollock, R., Harvey, J., Guilford, P., Doñate, F., Wilson, W.R., and Patterson, A.V. (2010). The bioreductive prodrug PR-104A is activated under aerobic conditions by human aldo-keto reductase $1 \mathrm{C} 3$. Cancer Res. 70, 1573-1584.

Gumulya, Y., and Gillam, E.M.J. (2017). Exploring the past and the future of protein evolution with ancestral sequence reconstruction: the "retro" approach to protein engineering. Biochem. J. 474, 1-19.

Guo, T., Cui, L., Shen, J., Zhu, W., Xu, Y., and Qian, X. (2013). A highly sensitive long-wavelength fluorescence probe for nitroreductase and hypoxia: selective detection and quantification. Chem. Commun. (Camb.) 49, 10820-10822.

Haberkorn, U., Oberdorfer, F., Gebert, J., Morr, I., Haack, K., Weber, K., Lindauer, M., van Kaick, G., and Schackert, H.K. (1996). Monitoring gene therapy with cytosine deaminase: in vitro studies using tritiated-5fluorocytosine. J. Nucl. Med. 37, 87-94.

Hamstra, D.A., Rice, D.J., Fahmy, S., Ross, B.D., and Rehemtulla, A. (1999). Enzyme/prodrug therapy for head and neck cancer using a catalytically superior cytosine deaminase. Hum. Gene Ther. 10, 1993-2003.

Hanahan, D., and Weinberg, R.A. (2011). Hallmarks of cancer: the next generation. Cell 144, 646-674.

Hannink, N., Rosser, S.J., French, C.E., Basran, A., Murray, J.A., Nicklin, S., and Bruce, N.C. (2001). Phytodetoxification of TNT by transgenic plants expressing a bacterial nitroreductase. Nat. Biotechnol. 19, 1168-1172.

Heap, J.T., Pennington, O.J., Cartman, S.T., Carter, G.P., and Minton, N.P. (2007). The ClosTron: a universal gene knock-out system for the genus Clostridium. J. Microbiol. Methods 70, 452-464.

Heap, J.T., Theys, J., Ehsaan, M., Kubiak, A.M., Dubois, L., Paesmans, K., Mellaert, L.V., Knox, R., Kuehne, S.A., Lambin, P., and Minton, N.P. (2014). Spores of Clostridium engineered for clinical efficacy and safety cause regression and cure of tumors in vivo. Oncotarget 5, 1761-1769. 
Helsby, N.A., Ferry, D.M., Patterson, A.V., Pullen, S.M., and Wilson, W.R. (2004). 2-Amino metabolites are key mediators of CB 1954 and SN 23862 bystander effects in nitroreductase GDEPT. Br. J. Cancer 90, 1084-1092.

Heo, J., Reid, T., Ruo, L., Breitbach, C.J., Rose, S., Bloomston, M., Cho, M., Lim, H.Y., Chung, H.C., Kim, C.W., Burke, J., Lencioni, R., Hickman, T., Moon, A., Lee, Y.S., Kim, M.K., Daneshmand, M., Dubois, K., Longpre, L., Ngo, M., Rooney, C., Bell, J.C., Rhee, B.G., Patt, R., Hwang, T.H., and Kirn, D.H. (2013). Randomized dose-finding clinical trial of oncolytic immunotherapeutic vaccinia JX-594 in liver cancer. Nat. Med. 19, 329-336.

Hermiston, T.W., and Kuhn, I. (2002). Armed therapeutic viruses: strategies and challenges to arming oncolytic viruses with therapeutic genes. Cancer Gene Ther. 9, 1022-1035.

Holohan, C., Van Schaeybroeck, S., Longley, D.B., and Johnston, P.G. (2013). Cancer drug resistance: an evolving paradigm. Nat. Rev. Cancer 13, 714-726.

Horvat, C.N. (2012). Development and applications of nitroreductase activated masked fluorophores. PhD Thesis. School of Biological Sciences, Victoria University of Wellington, Wellington.

Hunt, M.A., Li, D., Hay, M.P., Currie, M.J., Robinson, B.A., Patterson, A.V., and Dachs, G.U. (2012). Characterisation of enzyme prodrug gene therapy combinations in coated spheroids and vascular networks in vitro. J. Gene Med. $14,62-74$.

Hunter, F.W., Wouters, B.G., and Wilson, W.R. (2016). Hypoxia-activated prodrugs: paths forward in the era of personalised medicine. Br. J. Cancer 114, 1071-1077.

Imperi, F., Massai, F., Ramachandran Pillai, C., Longo, F., Zennaro, E., Rampioni, G., Visca, P., and Leoni, L. (2013). New life for an old drug: the anthelmintic drug niclosamide inhibits Pseudomonas aeruginosa quorum sensing. Antimicrob. Agents Chemother. 57, 996-1005.

Jaberipour, M., Vass, S.O., Guise, C.P., Grove, J.I., Knox, R.J., Hu, L., Hyde, E.I., and Searle, P.F. (2010). Testing double mutants of the enzyme nitroreductase for enhanced cell sensitisation to prodrugs: effects of combining beneficial single mutations. Biochem. Pharmacol. 79, 102-111.

Janion, C. (2008). Inducible SOS response system of DNA repair and mutagenesis in Escherichia coli. Int. J. Biol. Sci. 4, 338-344.

Jarrom, D., Jaberipour, M., Guise, C.P., Daff, S., White, S.A., Searle, P.F., and Hyde, E.I. (2009). Steady-state and stopped-flow kinetic studies of three Escherichia coli NfsB mutants with enhanced activity for the prodrug CB1954. Biochemistry 48, 7665-7672. 
Jenkins, T.C., Naylor, M.A., O’Neill, P., Threadgill, M.D., Cole, S., Stratford, I.J., Adams, G.E., Fielden, E.M., Suto, M.J., and Stier, M.A. (1990). Synthesis and evaluation of alpha-[[(2-haloethyl)amino]methyl]-2- nitro- $1 \mathrm{H}-$ imidazole-1-ethanols as prodrugs of alpha-[(1-aziridinyl)methyl]-2- nitro$1 \mathrm{H}$-imidazole-1-ethanol (RSU-1069) and its analogues which are radiosensitizers and bioreductively activated cytotoxins. J. Med. Chem. 33, 2603-2610.

Jenkins, T.F., Leggett, D.C., Miyares, P.H., Walsh, M.E., Ranney, T.A., Cragin, J.H., and George, V. (2001). Chemical signatures of TNT-filled land mines. Talanta 54, 501-513.

Jepras, R.I., Carter, J., Pearson, S.C., Paul, F.E., and Wilkinson, M.J. (1995). Development of a robust flow cytometric assay for determining numbers of viable bacteria. Appl. Environ. Microbiol. 61, 2696-2701.

Ju, K.S., and Parales, R.E. (2010). Nitroaromatic compounds, from synthesis to biodegradation. Microbiol. Mol. Biol. Rev. 74, 250-272.

Kakirde, K.S., Parsley, L.C., and Liles, M.R. (2010). Size does matter: application-driven approaches for soil metagenomics. Soil Biol. Biochem. 42, 1911-1923.

Karjoo, Z., Chen, X., and Hatefi, A. (2016). Progress and problems with the use of suicide genes for targeted cancer therapy. Adv. Drug Deliv. Rev. 99, 113-128.

Kasinskas, R.W., and Forbes, N.S. (2007). Salmonella typhimurium lacking ribose chemoreceptors localize in tumor quiescence and induce apoptosis. Cancer Res. 67, 3201-3209.

Keiler, K.C., and Sauer, R.T. (1996). Sequence determinants of C-terminal substrate recognition by the Tsp protease. J. Biol. Chem. 271, 2589-2593.

Keiler, K.C., Waller, P.R., and Sauer, R.T. (1996). Role of a peptide tagging system in degradation of proteins synthesized from damaged messenger RNA. Science 271, 990-993.

Keu, K.V., Witney, T.H., Yaghoubi, S., Rosenberg, J., Kurien, A., Magnusson, R., Williams, J., Habte, F., Wagner, J.R., Forman, S., Brown, C., AllenAuerbach, M., Czernin, J., Tang, W., Jensen, M.C., Badie, B., and Gambhir, S.S. (2017). Reporter gene imaging of targeted $\mathrm{T}$ cell immunotherapy in recurrent glioma. Sci. Transl. Med. 9, eaag2196.

Kieser, T. (2000). Practical Streptomyces Genetics (John Innes Foundation).

Kim, H.Y., and Song, H.G. (2005). Purification and characterization of $\mathrm{NAD}(\mathrm{P}) \mathrm{H}$-dependent nitroreductase I from Klebsiella sp. C1 and enzymatic transformation of 2,4,6-trinitrotoluene. Appl. Microbiol. Biotechnol. 68, $766-773$. 
Kim, H.Y., Bennett, G.N., and Song, H.G. (2002). Degradation of 2,4,6trinitrotoluene by Klebsiella sp. isolated from activated sludge. Biotechnol. Lett. 24, 2023-2028.

Kim, J.H., Oh, J.Y., Park, B.H., Lee, D.E., Kim, J.S., Park, H.E., Roh, M.S., Je, J.E., Yoon, J.H., Thorne, S.H., Kirn, D., and Hwang, T.H. (2006). Systemic armed oncolytic and immunologic therapy for cancer with JX-594, a targeted poxvirus expressing GM-CSF. Mol. Ther. 14, 361-370.

Kirn, D.H., and Thorne, S.H. (2009). Targeted and armed oncolytic poxviruses: a novel multi-mechanistic therapeutic class for cancer. Nat. Rev. Cancer 9, 64-71.

Klaidman, L.K., Leung, A.C., and Adams, J.D. (1995). High-performance liquid chromatography analysis of oxidized and reduced pyridine dinucleotides in specific brain regions. Anal. Biochem. 228, 312-317.

Knox, R.J., Friedlos, F., Jarman, M., and Roberts, J.J. (1988a). A new cytotoxic, DNA interstrand crosslinking agent, 5-(aziridin-1-yl)-4hydroxylamino-2-nitrobenzamide, is formed from 5-(aziridin-1-yl)-2,4dinitrobenzamide (CB 1954) by a nitroreductase enzyme in Walker carcinoma cells. Biochem. Pharmacol. 37, 4661-4669.

Knox, R.J., Boland, M.P., Friedlos, F., Coles, B., Southan, C., and Roberts, J.J. (1988b). The nitroreductase enzyme in Walker cells that activates 5(aziridin-1-yl)-2,4-dinitrobenzamide (CB 1954) to 5-(aziridin-1-yl)-4hydroxylamino-2-nitrobenzamide is a form of $\mathrm{NAD}(\mathrm{P}) \mathrm{H}$ dehydrogenase (quinone) (EC 1.6.99.2). Biochem. Pharmacol. 37, 4671-4677.

Knox, R.J., Friedlos, F., Marchbank, T., and Roberts, J.J. (1991). Bioactivation of CB 1954: reaction of the active 4-hydroxylamino derivative with thioesters to form the ultimate DNA-DNA interstrand crosslinking species. Biochem. Pharmacol. 42, 1691-1697.

Knox, R.J., Friedlos, F., Sherwood, R.F., Melton, R.G., and Anlezark, G.M. (1992). The bioactivation of 5-(aziridin-1-yl)-2,4-dinitrobenzamide (CB1954)-II: A comparison of an Escherichia coli nitroreductase and Walker DT diaphorase. Biochem. Pharmacol. 44, 2297-2301.

Koch, C.J., Hahn, S.M., Rockwell, K., Covey, J.M., McKenna, W.G., and Evans, S.M. (2001). Pharmacokinetics of EF5 [2-(2-nitro-1-H-imidazol-1-yl)-N(2,2,3,3,3-pentafluoropropyl) acetamide] in human patients: implications for hypoxia measurements in vivo by 2-nitroimidazoles. Cancer Chemother. Pharmacol. 48, 177-187.

Koch, C.J., Scheuermann, J.S., Divgi, C., Judy, K.D., Kachur, A.V., Freifelder, R., Reddin, J.S., Karp, J., Stubbs, J.B., Hahn, S.M., Driesbaugh, J., Smith, D., Prendergast, S., and Evans, S.M. (2010). Biodistribution and dosimetry of ${ }^{18} \mathrm{~F}$-EF5 in cancer patients with preliminary comparison of ${ }^{18} \mathrm{~F}-\mathrm{EF} 5$ uptake 
versus EF5 binding in human glioblastoma. Eur. J. Nucl. Med. Mol. Imaging 37, 2048-2059.

Koder, R.L., and Miller, A.F. (1998). Steady-state kinetic mechanism, stereospecificity, substrate and inhibitor specificity of Enterobacter cloacae nitroreductase. Biochim. Biophys. Acta 1387, 395-405.

Konopleva, M., Thall, P.F., Yi, C.A., Borthakur, G., Coveler, A., BuesoRamos, C., Benito, J., Konoplev, S., Gu, Y., Ravandi, F., Jabbour, E., Faderl, S., Thomas, D., Cortes, J., Kadia, T., Kornblau, S., Daver, N., Pemmaraju, N., Nguyen, H.Q., Feliu, J., Lu, H., Wei, C., Wilson, W.R., Melink, T.J., Gutheil, J.C., Andreeff, M., Estey, E.H., and Kantarjian, H. (2015). Phase I/II study of the hypoxia-activated prodrug PR104 in refractory/relapsed acute myeloid leukemia and acute lymphoblastic leukemia. Haematologica 100, 927-934.

Kraft, M.Y., Kochergin, P.M., Tsyganova, A.M., and Shlikhunova, V.S. (1989). Synthesis of metronidazole from ethylenediamine. Pharm. Chem. J. 23, 861863.

Krick, E.L., Sorenmo, K.U., Rankin, S.C., Cheong, I., Kobrin, B., Thornton, K., Kinzler, K.W., Vogelstein, B., Zhou, S., and Diaz, L.A. (2012). Evaluation of Clostridium novyi-NT spores in dogs with naturally occurring tumors. Am. J. Vet. Res. 73, 112-118.

Kristian Räty, J., Liimatainen, T., Unelma Kaikkonen, M., Gröhn, O., Jumani Airenne, K., and Ylä-Herttuala, S. (2007). Non-invasive imaging in gene therapy. Mol. Ther. 15, 1579-1586.

Krohn, K.A., Link, J.M., and Mason, R.P. (2008). Molecular imaging of hypoxia. J. Nucl. Med. 49 Suppl 2, 129S-48S.

Kubiak, A.M., and Minton, N.P. (2015). The potential of clostridial spores as therapeutic delivery vehicles in tumour therapy. Res. Microbiol. 166, 244254.

Kucerova, P., and Cervinkova, M. (2016). Spontaneous regression of tumour and the role of microbial infection--possibilities for cancer treatment.

Anticancer Drugs 27, 269-277.

Kuehne, S.A., and Minton, N.P. (2012). ClosTron-mediated engineering of Clostridium. Bioengineered 3, 247-254.

Kunst, F., Ogasawara, N., Moszer, I., Albertini, A.M., Alloni, G., Azevedo, V., Bertero, M.G., Bessières, P., Bolotin, A., Borchert, S., Borriss, R., Boursier, L., Brans, A., Braun, M., Brignell, S.C., Bron, S., Brouillet, S., Bruschi, C.V., Caldwell, B., Capuano, V., Carter, N.M., Choi, S.K., Cordani, J.J., Connerton, I.F., Cummings, N.J., Daniel, R.A., Denziot, F., Devine, K.M., Düsterhöft, A., Ehrlich, S.D., Emmerson, P.T., Entian, K.D., Errington, J., Fabret, C., Ferrari, E., Foulger, D., Fritz, C., Fujita, M., Fujita, Y., et al. (1997). The complete 
genome sequence of the gram-positive bacterium Bacillus subtilis. Nature 390, 249-256.

Kuo, C., Coquoz, O., Troy, T.L., Xu, H., and Rice, B.W. (2007). Threedimensional reconstruction of in vivo bioluminescent sources based on multispectral imaging. J. Biomed. Opt 12, 24007.

Kurumata, M., Takahashi, M., Sakamotoa, A., Ramos, J.L., Nepovim, A., Vanek, T., Hirata, T., and Morikawa, H. (2005). Tolerance to, and uptake and degradation of 2,4,6-trinitrotoluene (TNT) are enhanced by the expression of a bacterial nitroreductase gene in Arabidopsis thaliana. Z. Naturforsch., C, J. Biosci. 60, 272-278.

Kutty, R., and Bennett, G.N. (2005). Biochemical characterization of trinitrotoluene transforming oxygen-insensitive nitroreductases from Clostridium acetobutylicum ATCC 824. Arch. Microbiol. 184, 158-167.

Kwak, Y.H., Lee, D.S., and Kim, H.B. (2003). Vibrio harveyi nitroreductase is also a chromate reductase. Appl. Environ. Microbiol. 69, 4390-4395.

Lapi, S.E., Voller, T.F., and Welch, M.J. (2009). Positron emission tomography imaging of hypoxia. PET Clin. 4, 39-47.

Le, D.T., Wang-Gillam, A., Picozzi, V., Greten, T.F., Crocenzi, T., Springett, G., Morse, M., Zeh, H., Cohen, D., Fine, R.L., Onners, B., Uram, J.N., Laheru, D.A., Lutz, E.R., Solt, S., Murphy, A.L., Skoble, J., Lemmens, E., Grous, J., Dubensky, T., Brockstedt, D.G., and Jaffee, E.M. (2015). Safety and survival with GVAX pancreas prime and Listeria monocytogenes-expressing mesothelin (CRS-207) boost vaccines for metastatic pancreatic cancer. J. Clin. Oncol. 33, 1325-1333.

Lears, K.A., Parry, J.J., Andrews, R., Nguyen, K., Wadas, T.J., and Rogers, B.E. (2015). Adenoviral-mediated imaging of gene transfer using a somatostatin receptor-cytosine deaminase fusion protein. Cancer Gene Ther. 22, 215-221.

Leblond, F., Davis, S.C., Valdés, P.A., and Pogue, B.W. (2010). Pre-clinical whole-body fluorescence imaging: Review of instruments, methods and applications. J. Photochem. Photobiol. B, Biol. 98, 77-94.

Lee, C.H. (2012). Engineering bacteria toward tumor targeting for cancer treatment: current state and perspectives. Appl. Microbiol. Biotechnol. 93, 517-523.

Lehouritis, P., Springer, C., and Tangney, M. (2013). Bacterial-directed enzyme prodrug therapy. J. Control. Release 170, 120-131.

Lehtinen, J., Nuutila, J., and Lilius, E.M. (2004). Green fluorescent proteinpropidium iodide (GFP-PI) based assay for flow cytometric measurement of bacterial viability. Cytometry A 60, 165-172. 
Li, Y., Li, P.-K., Roberts, M.J., Arend, R.C., Samant, R.S., and Buchsbaum, D.J. (2014). Multi-targeted therapy of cancer by niclosamide: A new application for an old drug. Cancer Lett. 349, 8-14.

Li, Y., Sun, Y., Li, J., Su, Q., Yuan, W., Dai, Y., Han, C., Wang, Q., Feng, W., and Li, F. (2015a). Ultrasensitive near-infrared fluorescence-enhanced probe for in vivo nitroreductase imaging. J. Am. Chem. Soc. 137, 6407-6416.

Li, Z., He, X., Wang, Z., Yang, R., Shi, W., and Ma, H. (2015b). in vivo imaging and detection of nitroreductase in zebrafish by a new near-infrared fluorescence off-on probe. Biosens. Bioelectron. 63, 112-116.

Lieschke, G.J., and Currie, P.D. (2007). Animal models of human disease: zebrafish swim into view. Nat. Rev. Genet. 8, 353-367.

Lin-Chao, S., Chen, W.T., and Wong, T.T. (1992). High copy number of the pUC plasmid results from a Rom/Rop-suppressible point mutation in RNA II. Mol. Microbiol. 6, 3385-3393.

Liochev, S.I., Hausladen, A., and Fridovich, I. (1999). Nitroreductase A is regulated as a member of the soxRS regulon of Escherichia coli. Proc. Natl. Acad. Sci. U.S.A. 96, 3537-3539.

Little, R.F. (2015). Directed evolution and discovery of nitroreductase enzymes for targeted cell ablation. MSc Thesis. School of Biological Sciences, Victoria University of Wellington, Wellington.

Lomovskaya, O., Warren, M.S., Lee, A., Galazzo, J., Fronko, R., Lee, M., Blais, J., Cho, D., Chamberland, S., Renau, T., Leger, R., Hecker, S., Watkins, W., Hoshino, K., Ishida, H., and Lee, V.J. (2001). Identification and characterization of inhibitors of multidrug resistance efflux pumps in Pseudomonas aeruginosa: novel agents for combination therapy. Antimicrob. Agents Chemother. 45, 105-116.

London, J., and Knight, M. (1966). Concentrations of nicotinamide nucleotide coenzymes in micro-organisms. J. Gen. Microbiol. 44, 241-254.

van Loon, J., Janssen, M.H.M., Ollers, M., Aerts, H.J.W.L., Dubois, L., Hochstenbag, M., Dingemans, A.M.C., Lalisang, R., Brans, B., Windhorst, B., van Dongen, G.A., Kolb, H., Zhang, J., De Ruysscher, D., and Lambin, P. (2010). PET imaging of hypoxia using $\left[{ }^{18} \mathrm{~F}\right] \mathrm{HX} 4$ : a Phase I trial. Eur. J. Nucl. Med. Mol. Imaging 37, 1663-1668.

Lovering, A.L., Hyde, E.I., Searle, P.F., and White, S.A. (2001). The structure of Escherichia coli nitroreductase complexed with nicotinic acid: three crystal forms at 1.7 A, 1.8 A and 2.4 A resolution. J. Mol. Biol. 309, 203-213.

Lussier, F.X., Chambenoit, O., Côté, A., Hupé, J.F., Denis, F., Juteau, P., Beaudet, R., and Shareck, F. (2011). Construction and functional screening 
of a metagenomic library using a T7 RNA polymerase-based expression cosmid vector. J. Ind. Microbiol. Biotechnol. 38, 1321-1328.

Malekshah, O.M., Chen, X., Nomani, A., Sarkar, S., and Hatefi, A. (2016). Enzyme/prodrug systems for cancer gene therapy. Curr. Pharmacol. Rep. 2, 299-308.

Manna, S., Harman, A., Accari, J., and Barth, C. (2013). Altering the selection capabilities of common cloning vectors via restriction enzyme mediated gene disruption. BMC Res. Notes 6, 85.

Maxmen, A. (2017). Living therapeutics: Scientists genetically modify bacteria to deliver drugs. Nat. Med. 23, 5-7.

McCormack, E., Silden, E., West, R.M., Pavlin, T., Micklem, D.R., Lorens, J.B., Haug, B.E., Cooper, M.E., and Gjertsen, B.T. (2013). Nitroreductase, a nearinfrared reporter platform for in vivo time-domain optical imaging of metastatic cancer. Cancer Res. 73, 1276-1286.

McKeage, M.J., Gu, Y., Wilson, W.R., Hill, A., Amies, K., Melink, T.J., and Jameson, M.B. (2011). A Phase I trial of PR-104, a pre-prodrug of the bioreductive prodrug PR-104A, given weekly to solid tumour patients. BMC Cancer 11, 432.

McOsker, C.C., and Fitzpatrick, P.M. (1994). Nitrofurantoin: mechanism of action and implications for resistance development in common uropathogens. J. Antimicrob. Chemother. 33 Suppl A, 23-30.

Mellman, I., Coukos, G., and Dranoff, G. (2011). Cancer immunotherapy comes of age. Nature 480, 480-489.

Michel, B. (2005). After 30 years of study, the bacterial SOS response still surprises us. PLoS Biol. 3, e255.

Miller, J.H. (1972). Experiments in molecular genetics (Cold Spring Harbor, N.Y.: Cold Spring Harbor Laboratory).

Miller, M.W., Howes, H.L., Kasubick, R.V., and English, A.R. (1970).

Alkylation of 2-methyl-5-nitroimidazole. Some potent antiprotozoal agents. J. Med. Chem. 13, 849-852.

Mineta, T., Rabkin, S.D., Yazaki, T., Hunter, W.D., and Martuza, R.L. (1995). Attenuated multi-mutated herpes simplex virus-1 for the treatment of malignant gliomas. Nat. Med. 1, 938-943.

Ministry of Health (2016). Cancer: New registrations and deaths 2013 (Wellington, NZ: Wellington: Ministry of Health).

Morokutti, A., Lyskowski, A., Sollner, S., Pointner, E., Fitzpatrick, T.B., Kratky, C., Gruber, K., and Macheroux, P. (2005). Structure and function of 
YcnD from Bacillus subtilis, a flavin-containing oxidoreductase. Biochemistry 44, 13724-13733.

Morris, D.G., Feng, X., DiFrancesco, L.M., Fonseca, K., Forsyth, P.A., Paterson, A.H., Coffey, M.C., and Thompson, B. (2013). REO-001: A Phase I trial of percutaneous intralesional administration of reovirus type 3 dearing (Reolysin ${ }^{\circledR}$ ) in patients with advanced solid tumors. Invest. New Drugs 31, 696-706.

Mowday, A.M., Guise, C.P., Ackerley, D.F., Minton, N.P., Lambin, P., Dubois, L.J., Theys, J., Smaill, J.B., and Patterson, A.V. (2016a). Advancing Clostridia to clinical trial: past lessons and recent progress. Cancers (Basel) 8 .

Mowday, A.M., Ashoorzadeh, A., Williams, E.M., Copp, J.N., Silva, S., Bull, M.R., Abbattista, M.R., Anderson, R.F., Flanagan, J.U., Guise, C.P., Ackerley, D.F., Smaill, J.B., and Patterson, A.V. (2016b). Rational design of an AKR1C3-resistant analog of PR-104 for enzyme-prodrug therapy. Biochem. Pharmacol. 116, 176-187.

Neuwoehner, J., Schofer, A., Erlenkaemper, B., Steinbach, K., Hund-Rinke, T.K., and Eisentraeger, A. (2007). Toxicological characterization of 2,4,6trinitrotoluene, its transformation products, and two nitramine explosives. Environ. Toxicol. Chem. 26, 1090-1099.

Norman, A., Hestbjerg Hansen, L., and Sørensen, S.J. (2005). Construction of a ColD cda promoter-based SOS-green fluorescent protein whole-cell biosensor with higher sensitivity toward genotoxic compounds than constructs based on recA, umuDC, or sulA promoters. Appl. Environ. Microbiol. 71, 2338-2346.

Olekhnovich, I.N., Goodwin, A., and Hoffman, P.S. (2009). Characterization of the $\mathrm{NAD}(\mathrm{P}) \mathrm{H}$ oxidase and metronidazole reductase activities of the RdxA nitroreductase of Helicobacter pylori. FEBS J. 276, 3354-3364.

de Oliveira, I.M., Bonatto, D., and Henriques, J.A.P. (2010). Nitroreductases: enzymes with environmental, biotechnological and clinical importance. In Current Research, Technology and Education Topics in Applied Microbiology and Microbial Biotechnology, A. Mendez-Vilas, ed. (Formatex Research Centre), pp. 1008-1019.

Oue, S., Okamoto, A., Yano, T., and Kagamiyama, H. (1999). Redesigning the substrate specificity of an enzyme by cumulative effects of the mutations of non-active site residues. J. Biol. Chem. 274, 2344-2349.

Owen, J.G., Robins, K.J., Parachin, N.S., and Ackerley, D.F. (2012). A functional screen for recovery of 4'-phosphopantetheinyl transferase and associated natural product biosynthesis genes from metagenome libraries. Environ. Microbiol. 14, 1198-1209. 
Packer, M.S., and Liu, D.R. (2015). Methods for the directed evolution of proteins. Nat. Rev. Genet. 16, 379-394.

Palmer, D.H., Mautner, V., Mirza, D., Oliff, S., Gerritsen, W., van der Sijp, J.R.M., Hubscher, S., Reynolds, G., Bonney, S., Rajaratnam, R., Hull, D., Horne, M., Ellis, J., Mountain, A., Hill, S., Harris, P.A., Searle, P.F., Young, L.S., James, N.D., and Kerr, D.J. (2004). Virus-directed enzyme prodrug therapy: intratumoral administration of a replication-deficient adenovirus encoding nitroreductase to patients with resectable liver cancer. J. Clin. Oncol. 22, 1546-1552.

Parachin, N.S., and Gorwa-Grauslund, M.F. (2011). Isolation of xylose isomerases by sequence- and function-based screening from a soil metagenomic library. Biotechnol Biofuels 4, 9.

Parato, K.A., Senger, D., Forsyth, P.A.J., and Bell, J.C. (2005). Recent progress in the battle between oncolytic viruses and tumours. Nat. Rev. Cancer 5, 965-976.

Parato, K.A., Breitbach, C.J., Le Boeuf, F., Wang, J., Storbeck, C., Ilkow, C., Diallo, J.S., Falls, T., Burns, J., Garcia, V., Kanji, F., Evgin, L., Hu, K., Paradis, F., Knowles, S., Hwang, T.-H., Vanderhyden, B.C., Auer, R., Kirn, D.H., and Bell, J.C. (2012). The oncolytic poxvirus JX-594 selectively replicates in and destroys cancer cells driven by genetic pathways commonly activated in cancers. Mol. Ther. 20, 749-758.

Patel, P., Young, J.G., Mautner, V., Ashdown, D., Bonney, S., Pineda, R.G., Collins, S.I., Searle, P.F., Hull, D., Peers, E., Chester, J., Wallace, D.M., Doherty, A., Leung, H., Young, L.S., and James, N.D. (2009). A Phase I/II clinical trial in localized prostate cancer of an adenovirus expressing nitroreductase with CB1954. Mol. Ther. 17, 1292-1299.

Paterson, E.S., Boucher, S.E., and Lambert, I.B. (2002). Regulation of the $n f s A$ gene in Escherichia coli by SoxS. J. Bacteriol. 184, 51-58.

Patyar, S., Joshi, R., Byrav, D.S.P., Prakash, A., Medhi, B., and Das, B.K. (2010). Bacteria in cancer therapy: a novel experimental strategy. J. Biomed. Sci. 17, 21.

Pavelka, A., Chovancova, E., and Damborsky, J. (2009). HotSpot Wizard: a web server for identification of hot spots in protein engineering. Nucleic Acids Res. 37, W376-383.

Pedada, S.R., Satam, V.S., Tambade, P.J., Kandadai, S.A., Hindupur, R.M., Pati, H.N., Launay, D., and Martin, D. (2013). An improved kilogram-scale synthesis of 2-bromo-4-nitro-1H-imidazole: a key building block of nitroimidazole drugs. Org. Process Res. Dev. 17, 1149-1155. 
Penet, M.F., Chen, Z., Li, C., Winnard, P.T., and Bhujwalla, Z.M. (2012). Prodrug enzymes and their applications in image-guided therapy of cancer: tracking prodrug enzymes to minimize collateral damage. Drug Deliv. Transl. Res. 2, 22-30.

Pérez-Reinado, E., Roldán, M.D., Castillo, F., and Moreno-Vivián, C. (2008). The NprA nitroreductase required for 2,4-dinitrophenol reduction in Rhodobacter capsulatus is a dihydropteridine reductase. Environ. Microbiol. 10, 3174-3183.

Piatkevich, K.D., and Verkhusha, V.V. (2011). Guide to red fluorescent proteins and biosensors for flow cytometry. Methods Cell Biol. 102, 431461.

Pisharath, H., Rhee, J.M., Swanson, M.A., Leach, S.D., and Parsons, M.J. (2007). Targeted ablation of beta cells in the embryonic zebrafish pancreas using E. coli nitroreductase. Mech. Dev. 124, 218-229.

Pitsawong, W., Hoben, J.P., and Miller, A.F. (2014). Understanding the broad substrate repertoire of nitroreductase based on its kinetic mechanism. J. Biol. Chem. 289, 15203-15214.

Poelwijk, F.J., Kiviet, D.J., Weinreich, D.M., and Tans, S.J. (2007). Empirical fitness landscapes reveal accessible evolutionary paths. Nature 445, 383386.

Pol, J., Buqué, A., Aranda, F., Bloy, N., Cremer, I., Eggermont, A., Erbs, P., Fucikova, J., Galon, J., Limacher, J.M., Preville, X., Sautès-Fridman, C., Spisek, R., Zitvogel, L., Kroemer, G., and Galluzzi, L. (2016). Trial WatchOncolytic viruses and cancer therapy. Oncoimmunology 5, e1117740.

Portsmouth, D., Hlavaty, J., and Renner, M. (2007). Suicide genes for cancer therapy. Mol. Aspects Med. 28, 4-41.

Prosser, G.A. (2011). Discovery and optimisation of bacterial nitroreductases for use in anti-cancer gene therapy. PhD Thesis. School of Biological Sciences, Victoria University of Wellington, Wellington.

Prosser, G.A., Copp, J.N., Syddall, S.P., Williams, E.M., Smaill, J.B., Wilson, W.R., Patterson, A.V., and Ackerley, D.F. (2010a). Discovery and evaluation of Escherichia coli nitroreductases that activate the anti-cancer prodrug CB1954. Biochem. Pharmacol. 79, 678-687.

Prosser, G.A., Patterson, A.V., and Ackerley, D.F. (2010b). uvrB gene deletion enhances SOS chromotest sensitivity for nitroreductases that preferentially generate the 4-hydroxylamine metabolite of the anti-cancer prodrug CB1954. J. Biotechnol. 150, 190-194.

Prosser, G.A., Copp, J.N., Mowday, A.M., Guise, C.P., Syddall, S.P., Williams, E.M., Horvat, C.N., Swe, P.M., Ashoorzadeh, A., Denny, W.A., Smaill, J.B., 
Patterson, A.V., and Ackerley, D.F. (2013). Creation and screening of a multi-family bacterial oxidoreductase library to discover novel nitroreductases that efficiently activate the bioreductive prodrugs CB1954 and PR-104A. Biochem. Pharmacol. 85, 1091-1103.

Prosser, G.A., Williams, E.M., Sissons, J.A., Walmsley, K.E., Parker, M.R., and Ackerley, D.F. (2015). A gain-of-function positive-selection expression plasmid that enables high-efficiency cloning. Biotechnol. Lett. 37, 383-389.

Quillardet, P., Huisman, O., D’Ari, R., and Hofnung, M. (1982). SOS chromotest, a direct assay of induction of an SOS function in Escherichia coli K-12 to measure genotoxicity. Proc. Natl. Acad. Sci. U.S.A. 79, 5971-5975.

Race, P.R., Lovering, A.L., White, S.A., Grove, J.I., Searle, P.F., Wrighton, C.W., and Hyde, E.I. (2007). Kinetic and structural characterisation of Escherichia coli nitroreductase mutants showing improved efficacy for the prodrug substrate CB1954. J. Mol. Biol. 368, 481-492.

Rafii, F., and Cerniglia, C.E. (1995). Reduction of azo dyes and nitroaromatic compounds by bacterial enzymes from the human intestinal tract. Environ. Health Perspect. 103 Suppl 5, 17-19.

Rainov, N.G. (2000). A Phase III clinical evaluation of herpes simplex virus type 1 thymidine kinase and ganciclovir gene therapy as an adjuvant to surgical resection and radiation in adults with previously untreated glioblastoma multiforme. Hum. Gene Ther. 11, 2389-2401.

Rajamuthiah, R., Fuchs, B.B., Conery, A.L., Kim, W., Jayamani, E., Kwon, B., Ausubel, F.M., and Mylonakis, E. (2015). Repurposing salicylanilide anthelmintic drugs to combat drug resistant Staphylococcus aureus. PLoS ONE 10, e0124595.

Rajendran, J.G., Schwartz, D.L., O’Sullivan, J., Peterson, L.M., Ng, P., Scharnhorst, J., Grierson, J.R., and Krohn, K.A. (2006). Tumor hypoxia imaging with [F-18] fluoromisonidazole positron emission tomography in head and neck cancer. Clin. Cancer Res. 12, 5435-5441.

Ramesh, N., Ge, Y., Ennist, D.L., Zhu, M., Mina, M., Ganesh, S., Reddy, P.S., and Yu, D.C. (2006). CG0070, a conditionally replicating granulocyte macrophage colony-stimulating factor-armed oncolytic adenovirus for the treatment of bladder cancer. Clin. Cancer Res. 12, 305-313.

Reetz, M.T., Kahakeaw, D., and Lohmer, R. (2008). Addressing the numbers problem in directed evolution. Chembiochem 9, 1797-1804.

Rich, M.H. (2012). Directed evolution of nitroreductase enzymes to enhance activation of a novel PET probe. Honours Thesis. School of Biological Sciences, Victoria University of Wellington, Wellington. 
Roberts, N.J., Zhang, L., Janku, F., Collins, A., Bai, R.Y., Staedtke, V., Rusk, A.W., Tung, D., Miller, M., Roix, J., Khanna, K.V., Murthy, R., Benjamin, R.S., Helgason, T., Szvalb, A.D., Bird, J.E., Roy-Chowdhuri, S., Zhang, H.H., Qiao, Y., Karim, B., McDaniel, J., Elpiner, A., Sahora, A., Lachowicz, J., Phillips, B., Turner, A., Klein, M.K., Post, G., Diaz, L.A., Riggins, G.J., Papadopoulos, N., Kinzler, K.W., Vogelstein, B., Bettegowda, C., Huso, D.L., Varterasian, M., Saha, S., and Zhou, S. (2014). Intratumoral injection of Clostridium novyi-NT spores induces antitumor responses. Sci Transl Med 6, 249ra111.

Robins, K.J., Hooks, D.O., Rehm, B.H.A., and Ackerley, D.F. (2013). Escherichia coli NemA is an efficient chromate reductase that can be biologically immobilized to provide a cell free system for remediation of hexavalent chromium. PLoS One 8, e59200.

Roldán, M.D., Pérez-Reinado, E., Castillo, F., and Moreno-Vivián, C. (2008). Reduction of polynitroaromatic compounds: the bacterial nitroreductases. FEMS Microbiol. Rev. 32, 474-500.

Romero, P.A., and Arnold, F.H. (2009). Exploring protein fitness landscapes by directed evolution. Nat. Rev. Mol. Cell Biol. 10, 866-876.

Roulstone, V., Khan, K., Pandha, H.S., Rudman, S., Coffey, M., Gill, G.M., Melcher, A.A., Vile, R., Harrington, K.J., de Bono, J., and Spicer, J. (2015). Phase I trial of cyclophosphamide as an immune modulator for optimizing oncolytic reovirus delivery to solid tumors. Clin. Cancer Res. 21, 1305-1312.

Russell, S.J., Peng, K.W., and Bell, J.C. (2012). Oncolytic virotherapy. Nat. Biotechnol. 30, 658-670.

Saadatpour, Z., Bjorklund, G., Chirumbolo, S., Alimohammadi, M., Ehsani, H., Ebrahiminejad, H., Pourghadamyari, H., Baghaei, B., Mirzaei, H.R., Sahebkar, A., Mirzaei, H., and Keshavarzi, M. (2016). Molecular imaging and cancer gene therapy. Cancer Gene Ther.

Salamanca-Pinzón, S.G., Camacho-Carranza, R., Hernández-Ojeda, S.L., and Espinosa-Aguirre, J.J. (2006). Nitrocompound activation by cell-free extracts of nitroreductase-proficient Salmonella typhimurium strains. Mutagenesis 21, 369-374.

Seymour, L.W., and Fisher, K.D. (2016). Oncolytic viruses: finally delivering. Br. J. Cancer 114, 357-361.

Shemiakina, I.I., Ermakova, G.V., Cranfill, P.J., Baird, M.A., Evans, R.A., Souslova, E.A., Staroverov, D.B., Gorokhovatsky, A.Y., Putintseva, E.V., Gorodnicheva, T.V., Chepurnykh, T.V., Strukova, L., Lukyanov, S., Zaraisky, A.G., Davidson, M.W., Chudakov, D.M., and Shcherbo, D. (2012). A monomeric red fluorescent protein with low cytotoxicity. Nat. Commun. 3, 1204. 
Shimotohno, A., Oue, S., Yano, T., Kuramitsu, S., and Kagamiyama, H. (2001). Demonstration of the importance and usefulness of manipulating non-active-site residues in protein design. J. Biochem. 129, 943-948.

Siim, B.G., Denny, W.A., and Wilson, W.R. (1997). Nitro reduction as an electronic switch for bioreductive drug activation. Oncol. Res. 9, 357-369.

Sisson, G., Goodwin, A., Raudonikiene, A., Hughes, N.J., Mukhopadhyay, A.K., Berg, D.E., and Hoffman, P.S. (2002). Enzymes associated with reductive activation and action of nitazoxanide, nitrofurans, and metronidazole in Helicobacter pylori. Antimicrob. Agents Chemother. 46, 2116-2123.

Slaney, C.Y., and Darcy, P.K. (2015). Releasing the brake on oncolytic viral therapy. Clin. Cancer Res. 21, 5417-5419.

Spiller, B., Gershenson, A., Arnold, F.H., and Stevens, R.C. (1999). A structural view of evolutionary divergence. Proc. Natl. Acad. Sci. U.S.A. 96, 12305-12310.

Stanton, M., Cronin, M., Lehouritis, P., and Tangney, M. (2015). In vivo bacterial imaging without engineering; A novel probe-based strategy facilitated by endogenous nitroreductase enzymes. Curr. Gene Ther. 15, 277-288.

Steinberg, B., and Ostermeier, M. (2016). Environmental changes bridge evolutionary valleys. Sci. Adv. 2, e1500921.

Stratford, I.J., Williamson, C., Hoe, S., and Adams, G.E. (1981).

Radiosensitizing and cytotoxicity studies with CB 1954 (2,4-dinitro-5aziridinylbenzamide). Radiat. Res. 88, 502-509.

Studier, F.W., and Moffatt, B.A. (1986). Use of bacteriophage T7 RNA polymerase to direct selective high-level expression of cloned genes. J. Mol. Biol. 189, 113-130.

Swe, P.M., Copp, J.N., Green, L.K., Guise, C.P., Mowday, A.M., Smaill, J.B., Patterson, A.V., and Ackerley, D.F. (2012). Targeted mutagenesis of the Vibrio fischeri flavin reductase FRase I to improve activation of the anticancer prodrug CB1954. Biochem. Pharmacol. 84, 775-783.

Swint-Kruse, L., Zhan, H., Fairbanks, B.M., Maheshwari, A., and Matthews, K.S. (2003). Perturbation from a distance: mutations that alter LacI function through long-range effects. Biochemistry 42, 14004-14016.

Sylvia, J.M., Janni, J.A., Klein, J.D., and Spencer, K.M. (2000). Surfaceenhanced raman detection of 2,4-dinitrotoluene impurity vapor as a marker to locate landmines. Anal. Chem. 72, 5834-5840. 
Taddei, F., Matic, I., and Radman, M. (1995). cAMP-dependent SOS induction and mutagenesis in resting bacterial populations. Proc. Natl. Acad. Sci. U.S.A. 92, 11736-11740.

Takeda, K., Sato, J., Goto, K., Fujita, T., Watanabe, T., Abo, M., Yoshimura, E., Nakagawa, J., Abe, A., Kawasaki, S., and Niimura, Y. (2010). Escherichia coli ferredoxin-NADP+ reductase and oxygen-insensitive nitroreductase are capable of functioning as ferric reductase and of driving the Fenton reaction. Biometals 23, 727-737.

Tanner, J.J., Tu, S.C., Barbour, L.J., Barnes, C.L., and Krause, K.L. (1999). Unusual folded conformation of nicotinamide adenine dinucleotide bound to flavin reductase P. Protein Sci. 8, 1725-1732.

Tatsumi, K., Doi, T., Yoshimura, H., Koga, H., and Horiuchi, T. (1982). Oxygen-insensitive nitrofuran reductases in Salmonella typhimurium TA100. J. Pharmacobio-Dyn. 5, 423-429.

Tegel, H., Ottosson, J., and Hober, S. (2011). Enhancing the protein production levels in Escherichia coli with a strong promoter. FEBS J. 278, 729-739.

Terrón-González, L., Medina, C., Limón-Mortés, M.C., and Santero, E. (2013). Heterologous viral expression systems in fosmid vectors increase the functional analysis potential of metagenomic libraries. Sci. Rep. 3, 1107.

Theys, J., Pennington, O., Dubois, L., Anlezark, G., Vaughan, T., Mengesha, A., Landuyt, W., Anné, J., Burke, P.J., Dûrre, P., Wouters, B.G., Minton, N.P., and Lambin, P. (2006). Repeated cycles of Clostridium-directed enzyme prodrug therapy result in sustained antitumour effects in vivo. Br. J. Cancer $95,1212-1219$.

Thorwarth, D., Eschmann, S.-M., Scheiderbauer, J., Paulsen, F., and Alber, M. (2005). Kinetic analysis of dynamic ${ }^{18} \mathrm{~F}$-fluoromisonidazole PET correlates with radiation treatment outcome in head-and-neck cancer. BMC Cancer 5, 152.

Tjuvajev, J.G., Doubrovin, M., Akhurst, T., Cai, S., Balatoni, J., Alauddin, M.M., Finn, R., Bornmann, W., Thaler, H., Conti, P.S., and Blasberg, R.G. (2002). Comparison of radiolabeled nucleoside probes (FIAU, FHBG, and FHPG) for PET imaging of HSV1-tk gene expression. J. Nucl. Med. 43, 10721083.

Todo, T., Martuza, R.L., Rabkin, S.D., and Johnson, P.A. (2001). Oncolytic herpes simplex virus vector with enhanced MHC class I presentation and tumor cell killing. Proc. Natl. Acad. Sci. U.S.A. 98, 6396-6401. 
Todo, T., Tanaka, M., Ito, M., Ito, H., and Ino, Y. (2014). Clinical trials of a third-generation recombinant oncolytic HSV-1 in recurrent glioblastoma and olfactory neuroblastoma patients. Neuro. Oncol. 16, iii51.

Tomizawa, J. (1986). Control of ColE1 plasmid replication: binding of RNA I to RNA II and inhibition of primer formation. Cell 47, 89-97.

Toso, J.F., Gill, V.J., Hwu, P., Marincola, F.M., Restifo, N.P., Schwartzentruber, D.J., Sherry, R.M., Topalian, S.L., Yang, J.C., Stock, F., Freezer, L.J., Morton, K.E., Seipp, C., Haworth, L., Mavroukakis, S., White, D., MacDonald, S., Mao, J., Sznol, M., and Rosenberg, S.A. (2002). Phase I study of the intravenous administration of attenuated Salmonella typhimurium to patients with metastatic melanoma. J. Clin. Oncol. 20, 142152.

Tracewell, C.A., and Arnold, F.H. (2009). Directed enzyme evolution: climbing fitness peaks one amino acid at a time. Curr. Opin. Chem. Biol. 13, $3-9$.

Uchiyama, T., and Miyazaki, K. (2009). Functional metagenomics for enzyme discovery: challenges to efficient screening. Curr. Opin. Biotechnol. 20, 616622.

Van Dessel, N., Swofford, C.A., and Forbes, N.S. (2015). Potent and tumor specific: arming bacteria with therapeutic proteins. Ther. Deliv. 6, 385-399.

Van Dillewijn, P., Couselo, J.L., Corredoira, E., Delgado, A., Wittich, R.-M., Ballester, A., and Ramos, J.L. (2008). Bioremediation of 2,4,6-trinitrotoluene by bacterial nitroreductase expressing transgenic aspen. Environ. Sci.

Technol. 42, 7405-7410.

Varghese, A.J., and Whitmore, G.F. (1980). Binding to cellular macromolecules as a possible mechanism for the cytotoxicity of misonidazole. Cancer Res. 40, 2165-2169.

Vass, S.O., Jarrom, D., Wilson, W.R., Hyde, E.I., and Searle, P.F. (2009). E. coli NfsA: an alternative nitroreductase for prodrug activation gene therapy in combination with CB1954. Br. J. Cancer 100, 1903-1911.

de Visser, K.E., Eichten, A., and Coussens, L.M. (2006). Paradoxical roles of the immune system during cancer development. Nat. Rev. Cancer 6, 24-37.

Vorobyeva, A.G., Stanton, M., Godinat, A., Lund, K.B., Karateev, G.G., Francis, K.P., Allen, E., Gelovani, J.G., McCormack, E., Tangney, M., and Dubikovskaya, E.A. (2015). Development of a bioluminescent nitroreductase probe for preclinical imaging. PLoS ONE 10, e0131037.

Wang, G., and Maier, R.J. (2004). An NADPH quinone reductase of Helicobacter pylori plays an important role in oxidative stress resistance and host colonization. Infect. Immun. 72, 1391-1396. 
Wardman, P., and Clarke, E.D. (1976). One-electron reduction potentials of substituted nitroimidazoles measured by pulse radiolysis. J. Chem. Soc., Faraday Trans. 1 72, 1377-1390.

Weinreich, D.M., Delaney, N.F., Depristo, M.A., and Hartl, D.L. (2006). Darwinian evolution can follow only very few mutational paths to fitter proteins. Science 312, 111-114.

West, S.E., Schweizer, H.P., Dall, C., Sample, A.K., and Runyen-Janecky, L.J. (1994). Construction of improved Escherichia-Pseudomonas shuttle vectors derived from pUC18/19 and sequence of the region required for their replication in Pseudomonas aeruginosa. Gene 148, 81-86.

Whiteway, J., Koziarz, P., Veall, J., Sandhu, N., Kumar, P., Hoecher, B., and Lambert, I.B. (1998). Oxygen-insensitive nitroreductases: analysis of the roles of $n f s A$ and $n f s B$ in development of resistance to 5-nitrofuran derivatives in Escherichia coli. J. Bacteriol. 180, 5529-5539.

Wiedenmann, J., Ivanchenko, S., Oswald, F., Schmitt, F., Röcker, C., Salih, A., Spindler, K.-D., and Nienhaus, G.U. (2004). EosFP, a fluorescent marker protein with UV-inducible green-to-red fluorescence conversion. Proc. Natl. Acad. Sci. U.S.A. 101, 15905-15910.

Williams, E.M. (2013). Development of bacterial nitroreductase enzymes for noninvasive imaging in cancer gene therapy. PhD Thesis. School of Biological Sciences, Victoria University of Wellington, Wellington.

Williams, E.M., Copp, J.N., and Ackerley, D.F. (2014). Site-saturation mutagenesis by overlap extension PCR. Methods Mol. Biol. 1179, 83-101.

Williams, E.M., Little, R.F., Mowday, A.M., Rich, M.H., Chan-Hyams, J.V.E., Copp, J.N., Smaill, J.B., Patterson, A.V., and Ackerley, D.F. (2015).

Nitroreductase gene-directed enzyme prodrug therapy: insights and advances toward clinical utility. Biochem. J. 471, 131-153.

Wilson, W.R., and Hay, M.P. (2011). Targeting hypoxia in cancer therapy. Nat. Rev. Cancer 11, 393-410.

Xu, G., and McLeod, H.L. (2001). Strategies for enzyme/prodrug cancer therapy. Clin. Cancer Res. 7, 3314-3324.

Yaghoubi, S.S., and Gambhir, S.S. (2006). PET imaging of herpes simplex virus type 1 thymidine kinase (HSV1-tk) or mutant HSV1-sr39tk reporter gene expression in mice and humans using $\left[{ }^{18} \mathrm{~F}\right] \mathrm{FHBG}$. Nat. Protoc. 1, 30693075 .

Yaghoubi, S.S., Couto, M.A., Chen, C.C., Polavaram, L., Cui, G., Sen, L., and Gambhir, S.S. (2006). Preclinical safety evaluation of ${ }^{18} \mathrm{~F}-\mathrm{FHBG}$ : a PET reporter probe for imaging herpes simplex virus type 1 thymidine kinase (HSV1-tk) or mutant HSV1-sr39tk's expression. J. Nucl. Med. 47, 706-715. 
Yaghoubi, S.S., Campbell, D.O., Radu, C.G., and Czernin, J. (2012). Positron emission tomography reporter genes and reporter probes: gene and cell therapy applications. Theranostics 2, 374-391.

Yang, Y., Lin, J., and Wei, D. (2016). Heterologous overexpression and biochemical characterization of a nitroreductase from Gluconobacter oxydans 621H. Mol. Biotechnol.

Yin, H., Wood, T.K., and Smets, B.F. (2005). Reductive transformation of TNT by Escherichia coli: pathway description. Appl. Microbiol. Biotechnol. 67, 397-404.

Ying, W. (2008). NAD+/NADH and NADP+/NADPH in cellular functions and cell death: regulation and biological consequences. Antioxid. Redox Signal. 10, 179-206.

Yu, W., and Fang, H. (2007). Clinical trials with oncolytic adenovirus in China. Curr Cancer Drug Targets 7, 141-148.

Yunis, A.A. (1988). Chloramphenicol: relation of structure to activity and toxicity. Annu. Rev. Pharmacol. Toxicol. 28, 83-100.

Zenno, S., Saigo, K., Kanoh, H., and Inouye, S. (1994). Identification of the gene encoding the major NAD $(\mathrm{P}) \mathrm{H}$-flavin oxidoreductase of the bioluminescent bacterium Vibrio fischeri ATCC 7744. J. Bacteriol. 176, 35363543.

Zenno, S., Koike, H., Kumar, A.N., Jayaraman, R., Tanokura, M., and Saigo, K. (1996). Biochemical characterization of NfsA, the Escherichia coli major nitroreductase exhibiting a high amino acid sequence homology to Frp, a Vibrio harveyi flavin oxidoreductase. J. Bacteriol. 178, 4508-4514.

Zenno, S., Kobori, T., Tanokura, M., and Saigo, K. (1998). Purification and characterization of NfrA1, a Bacillus subtilis nitro/flavin reductase capable of interacting with the bacterial luciferase. Biosci. Biotechnol. Biochem. 62, 1978-1987.

Zhang, J., Kale, V., and Chen, M. (2015). Gene-directed enzyme prodrug therapy. AAPS J. 17, 102-110.

Zhao, H., and Zha, W. (2006). In vitro "sexual" evolution through the PCRbased staggered extension process (StEP). Nat Protoc 1, 1865-1871.

Zheng, Z., Li, Y., Zhang, X., Liu, P., Ren, J., Wu, G., Zhang, Y., Chen, Y., and $\mathrm{Li}, \mathrm{X}$. (2015). A Bacillus subtilis strain can reduce hexavalent chromium to trivalent and an $n f r A$ gene is involved. Int. Biodeterior. Biodegradation 97, 90-96. 
Zhou, X.X., and Lin, M.Z. (2013). Photoswitchable fluorescent proteins: ten years of colorful chemistry and exciting applications. Curr. Opin. Chem.

Biol. 17, 682-690. 